COO-3060-2

MITNE-130

\title{
USE OF GAMMA SPECTROSCOPY FOR NEUTRONIC ANALYSIS OF LMFBR BLANKETS
}

\author{
by \\ Chang-Sun Kang, Norman C. Rasmussen, Michael J. Driscoll
}

November, 1971

\begin{abstract}
Department of Nuclear Engineering
Massachusetts Institute of Technology

Cambridge, Massachusetts 02139
\end{abstract}

Contract AT(11-1)-3060

U.S. Atomic Energy Commission 


\section{DISCLAIMER}

This report was prepared as an account of work sponsored by an agency of the United States Government. Neither the United States Government nor any agency Thereof, nor any of their employees, makes any warranty, express or implied, or assumes any legal liability or responsibility for the accuracy, completeness, or usefulness of any information, apparatus, product, or process disclosed, or represents that its use would not infringe privately owned rights. Reference herein to any specific commercial product, process, or service by trade name, trademark, manufacturer, or otherwise does not necessarily constitute or imply its endorsement, recommendation, or favoring by the United States Government or any agency thereof. The views and opinions of authors expressed herein do not necessarily state or reflect those of the United States Government or any agency thereof. 


\section{DISCLAIMER}

Portions of this document may be illegible in electronic image products. Images are produced from the best available original document. 


\author{
I USE OF GAMMA SPECTROSCOPY FOR \\ NEUTRONIC ANALYSIS OF LMFBR BLANKETS \\ by \\ Chang-Sun Kang \\ Norman C. Rasmussen \\ Michael J. Driscoll
}

November, 1971

Department of Nuclear Engineering

Massachusetts Institute of Technology

Cambridge, Massachusetts 02139

$$
\begin{gathered}
\text { COO }-3060-2 \\
\text { MITNE }-130
\end{gathered}
$$

Contract

$\operatorname{AT}(11-1)-3060$

U. S. Atomic Energy Commission

\title{
NOTICE
}

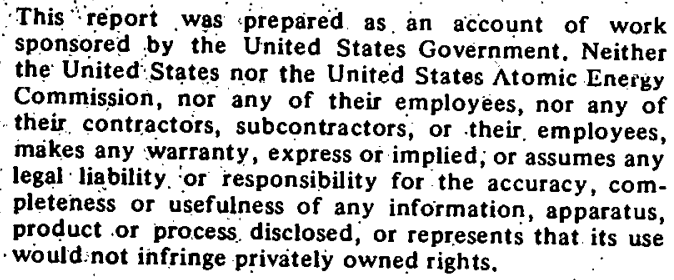




\section{DISTRIBUTION LIST \\ Contract AT (II - I) -3060}

U. S. Atomic Energy Commission, Headquarters

Division of Reactor Development and Technology Reactor Physics Branch

( 3 copies)

U. S. Atomic Energy Commission

Cambridge Office

(2 copies)

Dr. Paul Greebler, Manager

Nuclear Energy Division

Breeder Reactor Department

General Electric Compnay

310 DeGuigne Drive

Sunnyvale, California 94086

(1 copy)

Dr. Harry Morewitz, Manager

LMFBR Physics and Safety Projects

Atomics International

P.0. Box 309

Canoga Park, California 91305

(1 copy)

Mr. Malcolm Dyos, Manager

Nuclear Development, LMFBR Project

Westinghouse Electric Corporation

Advanced Reactors Division

Waltz Mill Site

P.0. Box 158

Madison, Pennsylvania 15663

(1 copy)

Dr. Robert Avery, Director

Reactor Physics Division

Argonne National Laboratory

9700 South Cass Avenue

Argonne, Illinois 60539

( I copy)

Dr. Charles A. Preskitt, Jr.

Mgr., Atomic and Nuclear Department

Gulf Radiation Technology

P.0. Box 608

San Diego, California 92112

(1 copy) 


\section{ABSTRACT}

It was the purpose of the present investigation to extend and apply Ge(Li) gamma-ray spectroscopy to the study of fast reactor blankets. The focal point for this research was the Blanket Test Facility at the MITR and Blanket No. 2, a realistic mockup of the blanketreflector region of a large liquid metal cooled fast breeder reactor.

It was found that $\mathrm{Ge}(\mathrm{Li})$ detectors can be simultaneously used as both high energy neutron spectrometers and continuous gamma-ray spectrometers. The broadened internal conversion spectral line at $691.4 \mathrm{KeV}$ has been analyzed for the former purpose, and the Compton recoil continuum has been analyzed and unfolded for the latter. This development makes the Ge( $\mathrm{Li}$ ) spectrometer an extremely valuable shield analysis tool.

The moisture content of the sodium chromate used in the blanket mockup has been confirmed to be less than $0.1 \mathrm{w} / \mathrm{O}$ by prompt activation analysis.

Prompt capture and inelastic gamma, and decay gamma spectra emitted by the blanket were also analyzed to perform a neutron balance with mixed results. The inability to resolve U-238 prompt capture gammas made it necessary to use the low energy Np-239 decay gammas, with the attendant uncertainties due to large selfshielding corrections. Lack of data on the variation of prompt gamma yield with neutron energy for all blanket constituents also contributed to the uncertainties, which together made it impossible to develop this method to the point where reliable practical application can be recommended: 


\section{ACKNOWLEDGEMENTS}

The work described in this report was made possible through the support of the M.I.T. LMFBR Blanket Physics Project which is sponsored by the United States Atomic Energy Commission. The same report has been also submitted by $C$. S. Kang in partial fulfillment of the requirements for the $\mathrm{ScD}$ degree at M.I.T.

Overall direction of this work has been shared by Professors M. J. Driscoll and N. C. Rasmussen. Special thanks are offered to Mr. Albert Supple, Dr. Yoshiyuti Hukai, Mr. Timothy Leung and Mr. Nestor Ortiz.:

Greatly acknowledged are the assistance, patience and cooperation of the M.I.T. Reactor Machine shop Staff, Radiation Protection Officers, Reactor Operation Personnel and M.I.T. Information Processing Center. Thanks are extended also to Miss Cindi Mitaras for the typing of this report. 
Abstract

Acknowledgements 3

List of Figures 8

List of Tables 11

Chapter I Introduction 13

Chapter II Experimental Apparatus $\quad 17$

2-I Blanket Test Facility _ 17

2-2 Ge(Li) Detectors and the Associated 20 Electronics

2-3 Energy Calibration Standards and $\quad 25$

Chapter III Determination of Neutron Reaction 30 Rates in LMFBR Blanket No. 2

3-1 Introduction 30

3-2 Theory 35

3-3 Prompt Capture Gamma-Rays 40

3-3-1 Total Efficiency 40

3-3-2 Data Analysis 43

3-4 Decay Gamma-Rays 49

3-4-I Induced Radioactivity in the 49

3-4-2 Correction Factors 53

3-4-3 Intrinsic Efficiency 58

3-4-4. Capture Reactions in $\mathrm{Na}$ and 64 
3-5 Inelastic Scattering Gamma-rays

67

3-5-I Inelastic Scattering Rates

67

3-5-2 Calculation of Total

Efficiency for Low Energy

Gamma-Rays

72

3-6 Fission in the Blanket

76

3-6-1 Short-Lived Fission Product

Gamma-Rays

3-6-2 Fission Rates

82

3-7 Summary

$-84$

Chapter IV Determination of Hydrogen Content of

91

LMFBR Blanket Materials

4-1 Introduction

91

4-2 Theory

94

4-3 Experimental Setup and Procedure 99

4-3-1 Irradiation Facility and 99

Detection Setup

4-3-2 Operating Procedure . 102

4-4 Data Analysis 103

Chapter $V$ Determination of the Neutron Leakage 109

Spectrum from Blanket No. 2

5-I Introduction : 109

5-2 Theory 110

5-2-1 Internal Conversion Spectrum 110 at $691.4 \mathrm{KeV}$

5-2-2 Broadening of $691.4 \mathrm{KeV}$ Spectrum

112

5-2-3 Integral Equation Formulation. 115 
5-2-4 Recoiling Kernel $\mathrm{P}\left(\mathrm{E} \rightarrow \mathrm{E}^{\prime}\right) \quad 118$

5-2-5 Response Diagonal Matrix and 120

5-3 Data Analys is . 123

5-3-1 Cf-252 Standard Neutron Source 123

5-3-2 Experimental Procedure and 124

5-4 Some Other Methods for Extracting 133

Neutron Spectra from Gamma-Ray Spectra

5-4-1 Energy Shift Method 133

5-4-2 Prompt Activation Method 136

Chapter VI Gamma-Ray Dosimetry 141

6-I Introduction 141

6-2 Theory 143

6-3 Data Analysis $\quad 147$

Chapter VII Conclusions and Recommendations 153

7-1 Conclusions 153

7-2 Recommendations $\quad 156$

References . 158

Appendix A Calculation of Blanket Axial Neutron 163

Flux

Appendix B Measurements of Gamma-Ray Linear 166

Attenuation Coefficients

Appendix C Calculation of Fast Neutron Capture $\quad 170$ Rate of Hydrogen

Appendix D Computer Program INTEF 173

Appendix E Calculation of Gamma-ray Self-Absorp- 191 tion Correction Factors for Blanket

No. 2 
E-1 Theory 191

E-2. Experimental Correction for Fuel 199 Heterogeneity

E-3 Computer Programs SELFN and 200 SELFU

Appendix F Computer Program SPECT

Appendix G Computer Program COMPT $\quad .250$

Appendix H Input Data of Runs No. 38, 39, . 291 and 40

Appendix I GAMANL Output Data and Their . - 295 Spectral Figures of: Runs No. 99, $102,103,104$ and 105 


\section{LIST OF FIGURES}

Figure

Page

2-1 Schematic Plan View of Blanket Test Facility 19 and Blanket No. 2

2-2 Blanket No. 2 Subassembly 21

2-3 Block Diagram of Detector Electronics for 24 Operation in the Free Mode

2-4 Linearity Correction as a Function of Channel Number for Free Mode Operation

3-1 Schematic Overall Plan View of Blanket _ 36 No. 2 and Detection Setup

3-2 Total Efficiency Curve of Detection System 42 for High Gamma-Energies

3-3 Self-Absorption Correction Factors for Gamma-Rays from $\mathrm{Na}_{2} \mathrm{CrO}_{4}$ of Blanket $\mathrm{No} .2$ (Calculated by SELFN)

3-4 Self-Absorption Correction Factors for Gamma-Rays from U-Fuel Rods of Blanket No. 2 (Calculated by SELFU)

3-5 Schematic View of the Setup for the Efficiency Measurements

3-6 Variation of Intrinsic Efficiency with 63 Distance from Detector

3-7 Intrinsic Efficiency of 17 c.c Ge(Li) 65 Detector.

3-8 Calculated Total Efficiency for Low Energy 73 Gamma-Rays, Which Originate from $\mathrm{Na}_{2} \mathrm{CrO}_{4}$

4-1 Percentage Error in Reaction Rates in Blanket 92 No. 2 as a Function of the Sodium Chromate Water Content Weight Percentage

4-2 Schematic of Sample Position in Front 101 Irradiation Facility 
4-3 Spectrum of $\mathrm{H}$ and $\mathrm{Cr}$ of $\mathrm{Na}_{2} \mathrm{CrO}_{4}$ with Known 104 Amount of Hydrogen Added, kun No. 38

4-4 Spectrum of $\mathrm{H}$ and $\mathrm{Cr}$ of $\mathrm{Na}_{2} \mathrm{CrO}_{4}$, Run No.40. 105

5-1 The Energy Lost to Ionization vs. the Recoil 114 Energy of Germanium

5-2 691.4 KeV Spectrum from Cf-252, Run No. $91 \quad 127$

5-3 Comparison of the Cf-252 Neutron Spectrum 129 with the Measured Collision Density Spectrum

5-4 Blanket $691.4 \mathrm{KeV} \mathrm{Ge}{ }^{72}$ Gamma Spectrum, $\quad 130$ Run No. 107

5-5. The Neutron Energy Spectrum Leaking from $\overline{1} 31$ Blanket No. 2, Run No. 107

5-6 The Neutron Energy Spectrum Leaking from 132 Blanket No. 2, Run No. 106.

6-1 Gamma-Ray Continuum from Blanket No. 2, 149 Run No. 105

6-2 The Gamma Ray Spectrum Leaking from Blanket 150 No. 2, Run No. 105

A-1 Total Axial Neutron Flux of Blanket No. 2164 Calculated by S8, 26-Group ANISN vs. Distance into blanket

E-1 Top View of the Part of Blanket No. 2 seen 192 by the Detector through the 2-in. diameter beam hole

E-2 Top View of Region A of Blanket No. 2 Seen 195 by the Detector, and a Unit Cell

E-3 Views of the Test Box and its Fuel Rod Array 201

E-4 Gamma-Ray Transmission Factor of a Unit Cell 202 of Fuel Rods

I-I Run No. 99, Short-Lived Gamma-Rays from 309 Blanket No. 2

I-2 Run No. 102, Short-Lived Gammas (280 min: 310 Cooling) 
I-3 Run No. 103, Short-Lived Gammas (25 hrs. Cooling)

I-4 Run No. 104-1, Prompt Gamma-Spectrum from Blanket No. 2

I-5 Run No. 104-2, Prompt Gamma-Spectrum from Blanket No. 2

I-6 Run No. 105-l, Prompt Gamma-Spectrum from Blanket No. 2

I-7 Run No. 105-2, Prompt Gamma-Spectrum from Blanket No. 2 
TABLE

2-1 Homogenized Atom Densities in B.T.F.

PAGE

Blanket No.

2-2 Energy Calibration Standards

28

3-1 Observable Prompt Gamma-Rays from

33

3-2 Observable Short-Lived Decay Gamma-

Rays from Blanket No. ?

3-3 Princ Łpal Background Gamma-Rays

44

3-4 Reaction Rate Calculations for Iron in Blanket No. 2, Runs No. 104 and 105

47

3-5. Reaction Rate Calculation for $\mathrm{Na}$ and $\mathrm{Cr}$ in Blanket No. 2, Runs No. 104 and 105

48

3-6 Standard Sources (New England Nuclear Corporation)

62

3-7 The Capture Rates of $\mathrm{U}-238$ and $\mathrm{Na}$ in

68 Blanket No. 2 Using Various Decay GammaRays from $\mathrm{Np}-239$ and $\mathrm{Na}-24$

3-8 Energy of First and Second Excited States 70

3-9 Calculation of Normalization Factor 75

3-10 Inelastic Gamma-Rays in Blanket No. 2, 77 Run No. 105

3-11 Short-Lived Fission Product Gamma-Rays from Blanket No. 2

3-12 Fission Product Decay Chains of Interest 81 to the Present Work

3-13 Principal Fission Product Gammas of 83 Interest

3-14 Fission Rate Calculations for Run No. 9985

3-15 Reaction Rates in Blanket No. 2.88 
3-16 Neutron Balance in Blanket No. 2

89

4-1 The Energy of the Hydrogen Thermal Neutron Capture Gamma-Ray Line

95

5-1 Cf-252 Fission Neutron Spectrum Para- 125 meters

B-1 Gamma-Ray Linear Attenuation Coeffi- $\quad 167$ cients of Uranium

H-1 Descriptions of Runs No. 38, 39 and $40 \quad 291$

I-1 Descriptions of Runs No. 99, 102, and $103 \overline{295}$

I-2 Descriptions of Runs No. 104 and $105 \quad 296$ 
Chapter I

INTRODUCTION

The development of high energy resolution lithiumdrifted germanium detectors for gamma-ray measurements is a significant experimental advance which has been widely applied. Considerable previous work using these detectors for gamma-ray spectroscopy has been carried out at MIT, including prompt-and decay-gamma activation analysis (H2), and physics and burnup analysis of thermal reactor fuel (OI, H3, SI). It is the purpose of the present investigation to extend and apply these techniques to the study of fast reactor blankets as part of AEC sponsored research in this area at MIT.

The focal point for the research is the Blanket Test Facility at the MITR, which is powered by the thermal neutron flux from the MITR thermal column. The present work is concerned exclusively with measurements in Blanket No. 2, a mockup of a typical large LMFBR blanket. The only modification to the blanket design necessary for these studies was insertion of a 2 -inch diameter beam hole through the blanket reflector. The experimental apparatus including Blanket No. 2 is described. in Chapter II.

The general concern of the reactor physicist is 
determination of the neutron reaction rates entering into the overall neutron balance; and the same objective is also paramount in the specific area of blanket physics research. Thus the first application investigated, as reported in Chapter III, is mainly concerned with the determination of neutron reaction rates in Blanket No. 2. These include the neutron capture rates, the inelastic scattering rates, and the fission rates. Both decay and prompt gamma-rays from Blanket No. 2 äre analyzed for this purpose. The major constituents contributing useful gamma-rays were found to be U-238, $\mathrm{Na}, \mathrm{Cr}, \mathrm{Fe}$, and $\mathrm{O}$.

One not entirely anticipated result worthy of note was the complete disappearance of the U-238 prompt capture line at $4.059 \mathrm{MeV}$, which is so prominent in thermal neutron spectra.

Investigations have recently attributed persistent errors uncovered in analysis of LMFBR critical experiments to unanticipated moisture in graphite used to simulate carbide fuel (L4, Pl). Small amounts of hydrogen can be important because it is so much better a moderator than any IMFBR constituent and can therefore soften the neutron energy spectrum. Since the water content of the LMFBR blanket materials could thereby have a significant effect upon the neutron energy 
spectrum in the blanket, it is very important to know exactly how much moisture is contained in its constituent materials. Chapter IV treats the determination of the moisture content of sodium chromate, which is the major potential carrier for moisture in Blanket No. 2. Prompt activation analysis with thermal neutrons was used for this analysis.

Chapter V treats the application of gamma-ray spectroscopy to determine the neutron energy spectrum leaking from Blanket No. 2. Three methods are considered in this chapter:

1) An energy shift method using low-Z materials,

2) A prompt activation method, and

3) The germanium atomic recoil method.

The third method is developed in detail and applied to measure the neutron energy spectrum from Blanket No. 2 .

In most experiments with $\mathrm{Ge}(\mathrm{Li})$ detectors for gamma-ray measurements, the dominance of the Compton recoil continuum in the detectors is regarded as an unwanted and troublesome complication. R. Gold (GI), however, showed that this feature can be exploited for continuous gamma-ray spectroscopy, and that it is therefore a useful tool for shielding analysis. Chapter VI is mainly concerned with the application of his 
method to analysis of the continuous gamma-ray spectra from Blanket No. 2. Use of a fairly large $\mathrm{Ge}(\mathrm{Li})$ crystal ( 17 c.c.) made possible analysis of very high energy gamma-rays (up to $10 \mathrm{MeV}$ ).

In general, then, Chapters III and IV are concerned with extension of conventional photo-peak analysis into the realm of LMFBR blanket applications, while chapters $\mathrm{V}$ and $\mathrm{VI}$ introduce some more novel capabilities of the $\mathrm{Ge}(\mathrm{Li})$ detector. In a sense these latter applications can be considered to be "free information" obtained in the conventionally oriented gamma-ray spectra from Blanket No. 2. They are, however, of considerable interest in their own right. Since we have now shown that it is possible to use the Ge(Li) detector as a simultaneous spectrometer for both gamma-rays and neutrons, it deserves increased consideration as a shield analysis tool.

In Chapter VII, this and other conclusions and recommendations are summarized. The bibliography follows immediately after, and data and computer programs are introduced in Appendices. 


\section{Chapter II \\ EXPERIMENTAL APPARATUS}

In this chapter the experimental apparatus concerned with the present work is discussed. First the Blanket Test Facility at the MITR is described, and the description of the $\mathrm{Ge}(\mathrm{Li})$ detectors and the associated electronics follows. Energy calibration and nonlinearity correction are discussed next.

2-1 Blanket Test Facility

The Blanket Test Facility (BTF) is located at the rear of the graphite-lined cavity at the end of the MITR thermal column. A detailed description of the BTF is given in Reference Fl. The front component in the $\mathrm{BTF}$ is a converter assembly, composed of a fission plate, filter and back scatter regions. Powered by the thermal neutron flux from the MITR thermal column, the converter generates a fast neutron flux similar to LMFBR core leakage spectra for testing mockups of LMFBR blankets. The converter assembly consists of a graphite backscatter-moderator region composed of 4 inch by 4 inch reactor grade graphite stringers, and a fuel region, which serves as both fission plate and filter, composed of 0.5-in. - diameter, aluminum clad $\mathrm{UO}_{2}$ fuel rods in a close-packed, triangular pitch array. The active 
fuel region is $48 \mathrm{in.} \mathrm{high} \mathrm{and} 60 \mathrm{in.}$ wide, and has U-235 enrichments of $1.0999 \%$ and $1.99 \%$.

The simulated blanket assembly is installed directly behind the converter assembly. BTF Blanket No. 2 is an accurate mock-up of a typical LMFBR blanket composition. A detailed description of Blanket No: 2 is given in reference Ll. Figure 2-1 shows the schematic plan view of the Blanket Test Facility with Blanket No. 2 in place. Blanket No. 2 is installed directly behind the converter assembly, which provides the simulated core leakage spectrum. Two 2-ft. - thick heavy concrete shield doors are used to close-off the irradiation cave containing the converter and blanket assemblies. A 2-in.-diameter hole, 4 in. below mid-plane, has been drilled through the blanket reflector to provide a beam hole for fast neutron and gamma-ray spectrum measurements. This beam hole is aligned with the holes through four masonite and steel laminated plugs in the port 12CHI penetrating the shield doors, so that the measurements can be done externally. Lead collimators containing various-sized holes have been made up to fit into the holes of the masonite and steel laminated plugs. Blanket No. 2 is composed of 25 subassemblies which contain steel-clad uranium metal fuel rods and anhydrous 


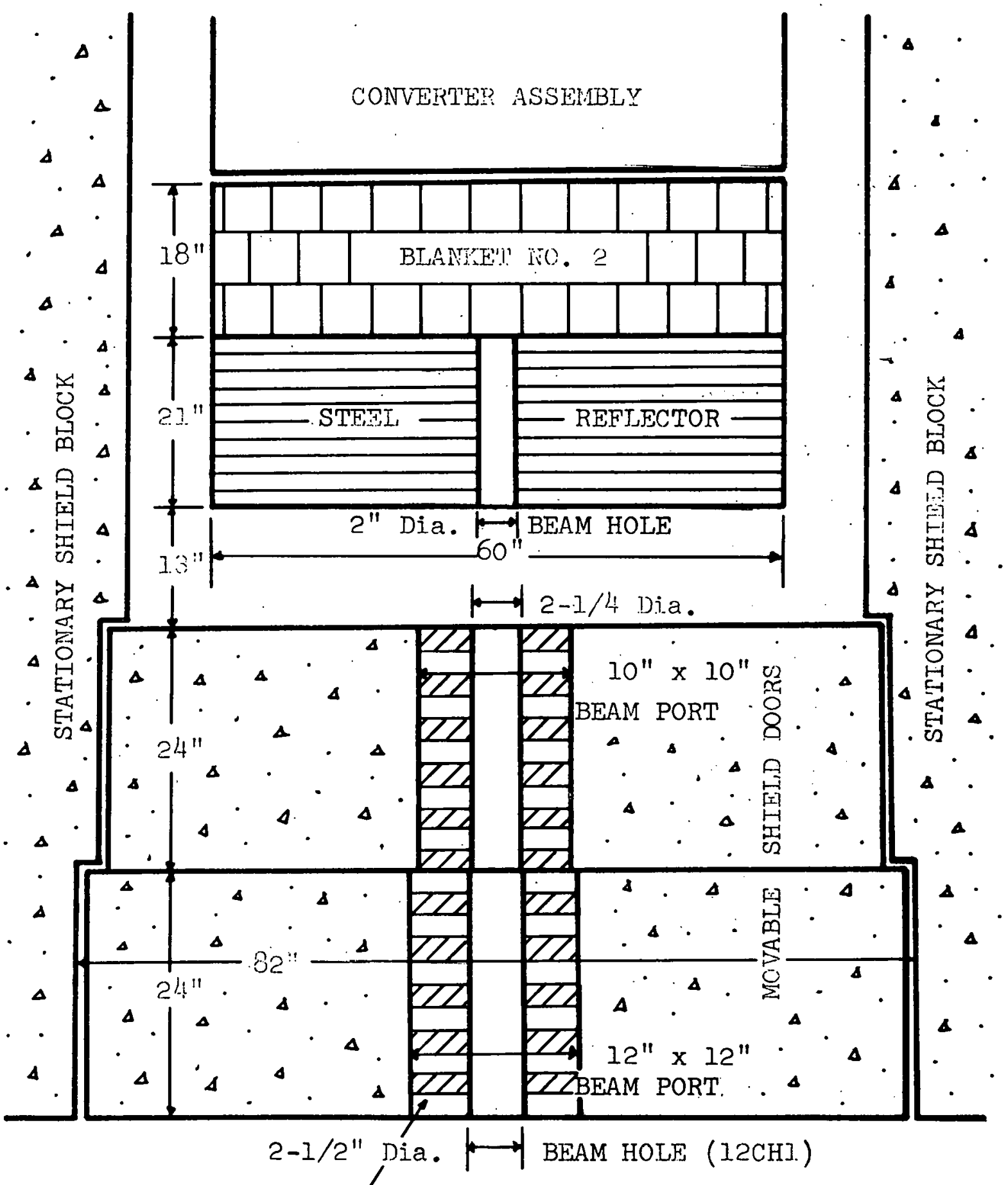

STEEL AND MASONITE LAMTNATED PLUGS

FIG. 2-I Schematic PJ.an Vj.ew o: Bianket Test

Facility and Biankes No. a 
sodium chromate powder (plus several outer subassemblies which are filled with a mixture of iron punchings and anhydrous borax powder). Each subassembly is loaded with 121 uranium metal fuel rods arranged on a square lattice spacing of 0.511 in.; the 1/4-in. - diameter uranium metal fuel is clad in low-carbon steel tubing. The inter-rod volume in each subassembly is filled with anhydrous sodium chromate $\left(\mathrm{Na}_{2} \mathrm{CrO}_{4}\right)$ powder. Figure 2-2 shows a cross-sectional view through a subassembly. The homogeneous atom densities in Blanket No. 2 are given in Table 2-I, where they are compared with the atom densities in a realistic blanket. As can be seen the homogenized compositions are in good agreement, confirming that Blanket No. 2 is a realistic mockup of an actual LMFBR blanket. 2-2 Ge (Li) Detectors and the Associated Electronics The main detector used in this work was a Ge( $\mathrm{Li}$ ) detector, which has been described by $V$. Rogers in Reference Rl. This detector has an active volume of 17 c.c. It has a relatively large dead region, the overall efficiency is fairly high, and it had a better energy resolution than any other detectors available to the author. The detector was operated at liquid nitrogen temperature $\left(77^{\circ} \mathrm{K}\right)$ with a reverse bias of $-700 \nabla$. The 

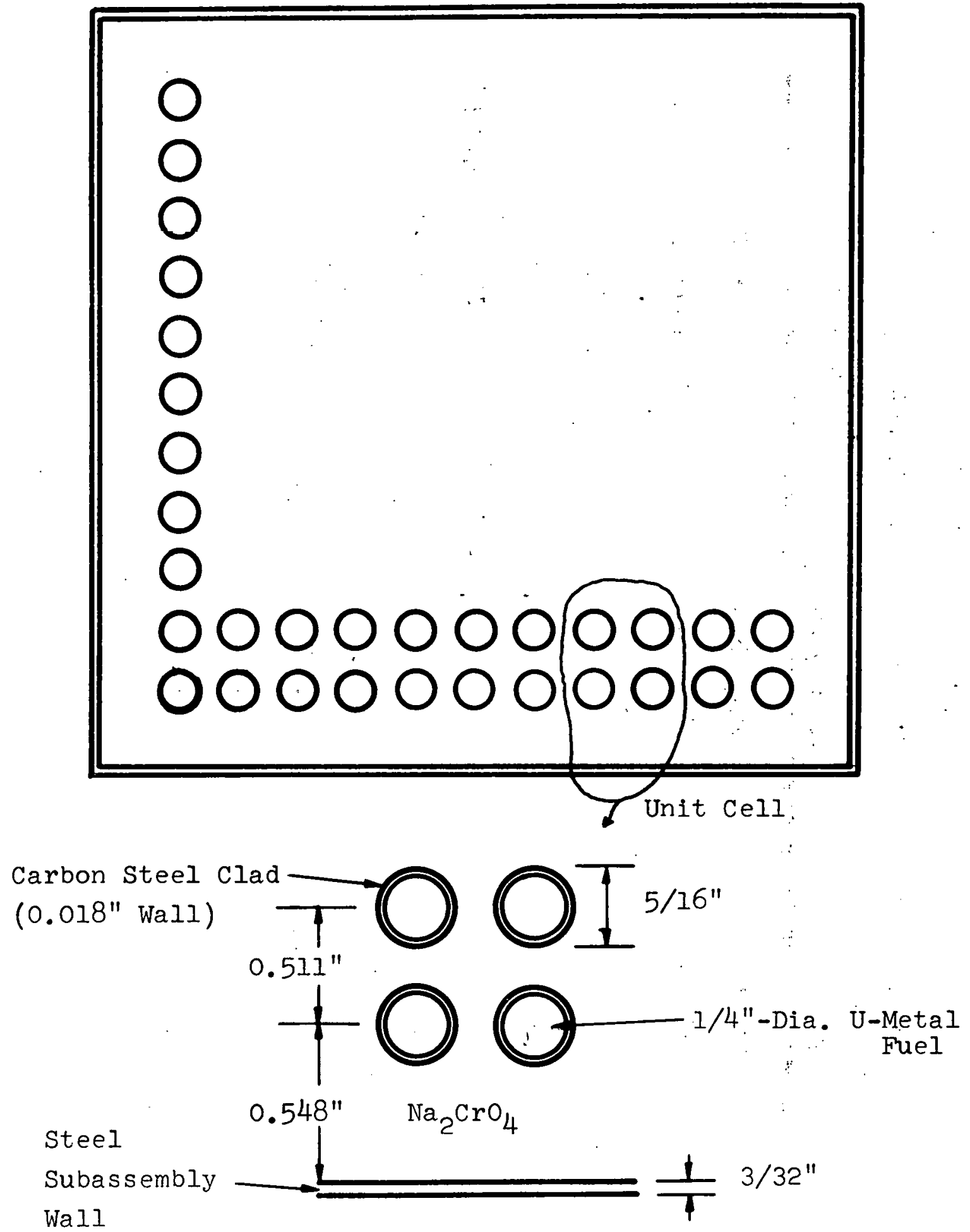

Fig. 2-2 Blanket No. 2 Subassembly 
TABLE 2-1

Homogenized Atom Densities in BTF Blanket No. 2

\begin{tabular}{|c|c|c|c|c|c|}
\hline Nuclide & . & Blanket No. & 2 & \multicolumn{2}{|c|}{$\begin{array}{c}\text { Equivalent Realistic* } \\
\text { Blanket }\end{array}$} \\
\hline$U-235$ & & 0.000088 & & 0.000016 & \\
\hline$U-238$ & & 0.008108 & & 0.008131 & \\
\hline 0 & & 0.016293 & & 0.016293 & \\
\hline $\mathrm{Na}$ & r & 0.008128 & & 0.008128 & \\
\hline $\mathrm{Cr}$ & & 0.0040647 & & $0.003728 \mathrm{j}$ & \\
\hline $\mathrm{Fe}$ & & 0.013750 & 0.017814 & 0.012611 & 0.017814 \\
\hline $\mathrm{Ni}$ & & C.000000 & & 0.001475 & \\
\hline $\mathrm{H}$ & & 0.000073 & $\theta^{\circ}$ & 0.000000 & \\
\hline $\mathrm{C}$ & & 0.000096 & & 0.000082 & \\
\hline
\end{tabular}

Reference: Ll

* Composed of $37.0 \mathrm{~V} / 0$ depleted $\mathrm{UO}_{2}$ (at $90 \%$ of theoretical density), $20.7 \mathrm{~V} / 0$ Type 316 stainiless steel, $32.0 \mathrm{~V} / 0$ sodium. and $10.3 \mathrm{~V} / 0$ void. 
exact bias voltage was determined by optimizing the energy resolution and the efficiency. The energy resolution of this detector varied from a full width at halt maximum, FWHM, of $3.5 \mathrm{KeV}$ to $7.0 \mathrm{KcV}$ acoording to the gamma-ray energies ( $511 \mathrm{KeV}$ and $7724 \mathrm{KeV}$, respectively). To reduce noise it is usually sufficient to have a solid electrical ground between the detector and the preamplifier. In particular it is strongly suggested. that the ground of the system be completely isolated from external electronic contacts. One has also to allow a certain period of time after one moves the detector or refills the dewar with liquid nitrogen in order to prevent boiling noise. Many otherconsiderations have been discussed in References $\mathrm{Ol}, \mathrm{H} 2, \mathrm{H} 3$ and $\mathrm{H} 4$. Generally there are three major methods of operation of the Ge(Li) spectrometer in the detection of gammarays: the direct or free mode, the Compton suppression mode and the pair spectrometer mode. The free mode method of operation, which was used throughout this work, consists. of using the detector and its associated electronics to drive the analyzer. No gating of the analyzer is done, and no coincidence or timing of the detected pulses is carried out. Figure 2-3 shows the schematic electronics 


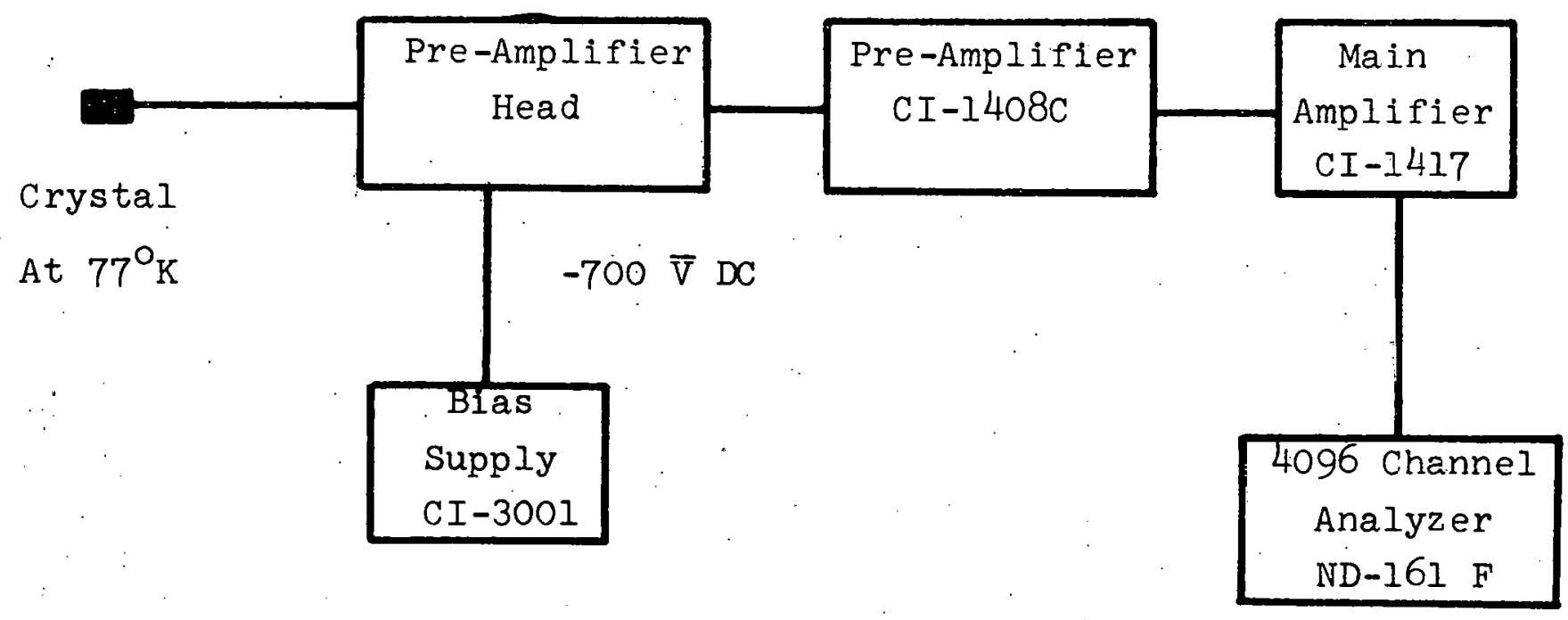

Fig. 2-3

BLOCK DIAGFAM OF DETECTOR

ELECTRONICS FOR OPERATION IN

THE FREE MODE 
diagram for free mode operation of the Ge(Li) detector. The free mode method of ope ration is preferable in the case of very low counting statistics. even though it incurs a higher background. 2-3 Energy Calibration Standards and Non-linearity Correction

In the present work two accurately known calibration peaks are used to obtain a linear fit between the channel number of the peak center and gamma-ray energy. Consequently the non-linearity of the detector and electronic amplifiers must be checked.

An experimental method is used to check for system non-linearity. This set-up consists of using a pulser connected to the test input of the CI-1408C preamplifier, to simulate a spectrum of many peaks equally spaced in energy. Analyzing such a spectrum gives an indication of the system energy linearity $(\mathrm{HI}, \mathrm{Ol})$. The method works well provided that the pulser is stable enough so as not to obscure the effect being measured. The pulser consists of a mercury relay precision pulse generator (RCL Model 20900), whose output amplitude is determined by the equally spaced triggering bias voltage provided by an EDC Model MV-IOON Precision Millivolt Standard (0-10 V output) with $0.01 \%$ accuracy. To increase pulse amplitude a $6 \boldsymbol{\nabla}$ standard dry cell is connected in series. A series 
of equally spaced pulses were analyzed for energy separation, and compared against what the separation would be if the system were perfectly linear. This analysis thus produced a set of linearity correction factors. The subroutine LINEAR of the computer code GAMANL (R2) performs this analysis. Figure $2-4$ shows a plot of these correction factors versus channel number obtained through the subroutine LINEAR for the various gain settings of the main amplifier. The linearity data was checked from time to time throughout these experiments, but the linearity correction data did not change noticably, as $V$. Orphan has also reported in Reference 07 .

The two known calibration peaks must be known very accurately to obtain a linear fit between the peak center and gamma-ray energy. Table 2-2 indicates the energy calibration standards used in the present work. The accuracies of the standards were $1.0 \mathrm{KeV}$ or better in all cases. Any pair of lines from Table 2-2 were used according to the situation. The electron annihilation line and the $\mathrm{Ar}^{41}$ decay line were prominent whenever the reactor was at power. In the case of decay gamma-ray analysis $\mathrm{Co}^{60}$ or $\mathrm{Na}^{24}$ lines were mainly used for the energy calibration. 


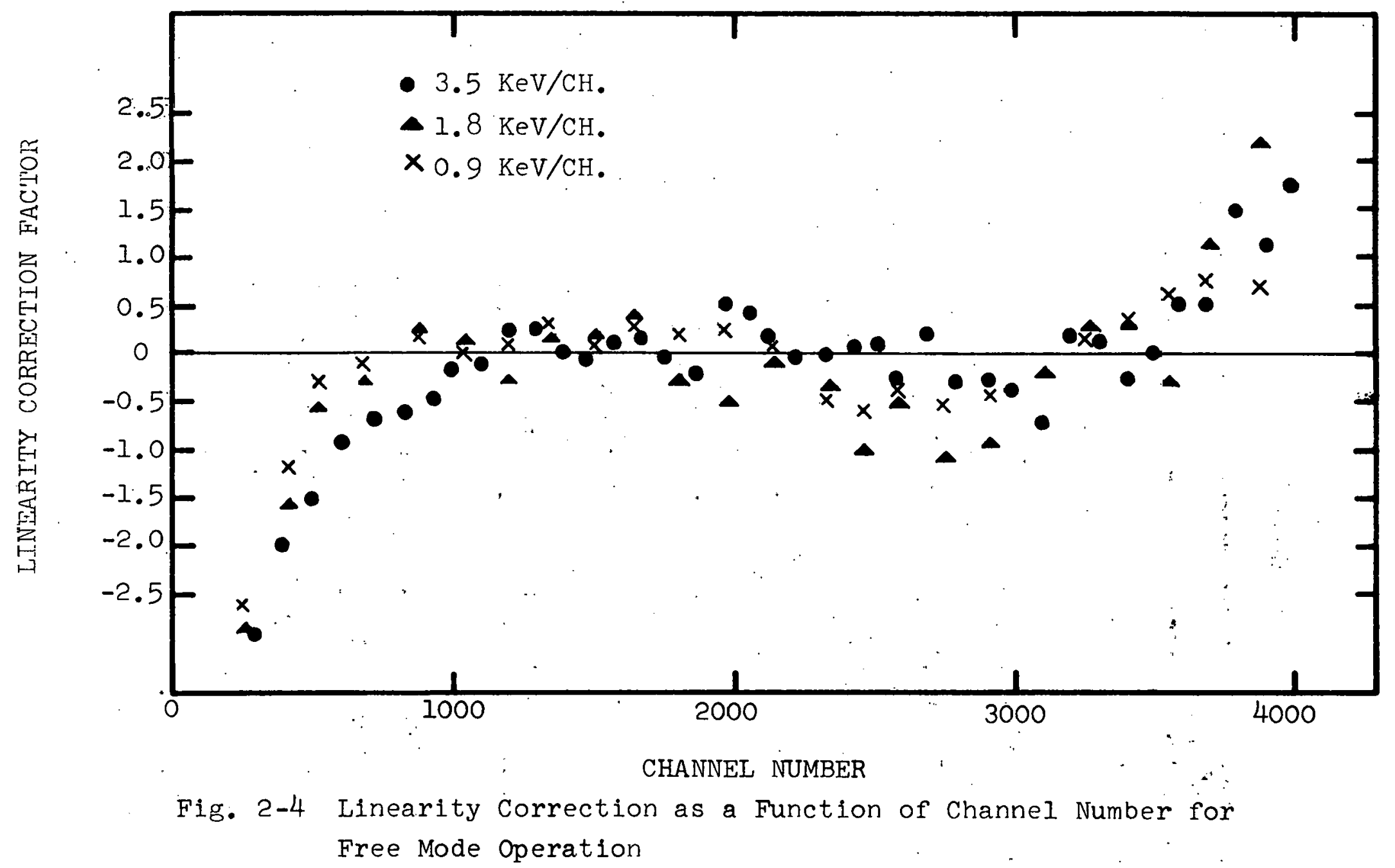


TABLE 2-2

ENERGY CALIBRATION STANDARDS

\begin{tabular}{ccc}
\hline ENERGY(KeV) & REMARK & REFERENCE \\
\hline $511.006 \pm 0.005$ & $\begin{array}{c}\text { Annihilation of } \\
\text { Electron-Positron }\end{array}$ & $\mathrm{a}$ \\
$1173.226 \pm 0.040$ & $\mathrm{Co}^{60}$ Decay & $\mathrm{b}$ \\
$1293.64 \pm 0.04$ & $\mathrm{Ar}^{41}$ Decay & $\mathrm{c}$ \\
$1332.48 \pm 0.05$ & $\mathrm{Co}^{60}$ Decay \\
$1368.53 \pm 0.04$ & $\mathrm{Na}^{24}$ Decay & $\mathrm{b}$ \\
$2753.92 \pm 0.12$ & $\mathrm{Na}^{24}$ Decay & $\mathrm{b}$ \\
$6018.2 \pm 1.0$ & Fe Capture Line & $\mathrm{d}$ \\
$7278.8 \pm 1.0$ & Fe Capture Line & $\mathrm{d}$ \\
\hline
\end{tabular}

References

a Electron rest mass from 1963 atomic constants.

b Murray G., et al., Nucl. Phys. G3 (1965) 353.

c White, D., et al., "Precision Measurements of GammaRays from $\mathrm{Co}^{60}, \mathrm{Ar}^{41}$, and $\mathrm{Cr}^{53}(\mathrm{n}, \gamma) \mathrm{Cr} 54 "$, Nucl. Ir.c. and Meth. 66 (1968) 70-76.

d Orphan V., et at., "Study of Thermal Neutron Capture Gamma-Rays Using a Ge(Li) Spectrometer"; MITNE-80 (Jan. 1970). 
Most data are analyzed through the computer code GAMANL(R2) and plotted using the CAL-COMP provided by the MIT Information Processing Center. The gamma-ray spectra from Blanket No. 2 will be analyzed in the following chapters, and, in particular, the reaction rates in Blanket No. 2 will: be discussed in the next chapter. 


\section{Chapter III \\ DETERMINATION OF NEUTRON REACTION \\ RATES IN LMFBR BLANKET NO. 2}

\section{3-1 Introduction}

In this chapter, Ge(Li) gamma-ray spectroscopy is

used to determine neutron reaction rates in the LMFBR $: \cdots$

blanket mockup, and thereby to deduce the neutron

balance in the blanket. These experimental results

will be compared with calculated values, other

experimental values, or both. Obtaining a variety

of independent experimental support for calculated

parameters is particularly important in the present

case because of the necessity for establishing credibility

for mockup experiments in lieu of studies on real

blankets.

The neutron reaction rates of interest may be categorized as capture, scattering, and fission rates.

The capture rate may be obtained by analyzing the prompt

capture gamma-rays and short-lived decay gamma-rays from

the blanket. Inelastic scattering rates, using the

characteristic prompt inelastic gamma-rays, and the

fission rate, using the short-lived fission product

decay gamma-rays, will also be analyzed in this chapter. 
Blanket No. 2 is mainly composed of $\mathrm{U}-238, \mathrm{Na}, \mathrm{Cr}, \mathrm{Fe}$, and 0 . By analyzing various gamma-rays from Blanket No. 2, one can determine the reaction rates of these major blanket constituents. The results can then be compared with the values obtained using foil activation methods. Through observation of the prompt capture gammarays emitted by Blanket No. 2, the information listed in Table 3-1 was found to be identifiable. Somewhat unexpectedly the major thermal neutron capture gamma-ray lines of U-238 were not observable in the spectra from Blanket No. 2 even though calculations and foil.irradia-. tions showed that over $90 \%$ of all captures were in $U-238$. Hukai identified more than 35 thermal neutron prompt : capture gamma-ray lines of U-238 in Reference H3. Groshev et al. (G2) also showed about 30 thermal neutron prompt capture gamma-ray lines of U-238 in the energy region of 3-4.5 MeV. In particular, the complete disappearance of the very prominent thermal neutron prompt capture gammaray line at $4.059 \mathrm{MeV}$ was hardly expected. Yost et al. (YI) have reported calculations which lead one to expect. . a decrease in the prompt capture gamma-ray yield of $\mathrm{U}-238$ at $4-4.25 \mathrm{MeV}$ by a factor of five. But the essentially complete disappearance observed for the 4.059 MeV line, and the other U-238 thermal capture 
lines as well is still unexplainable. This complete disappearance of the U-238 capture line at $4.059 \mathrm{KeV}$ made it difficult to deduce the capture rate of $\mathrm{U}-238$ in the blanket. However, observation of many short-lived decay gamma-ray lines from Np-239 provided an aiternate method for obtaining the capture rate of $\mathrm{U}-238$ (Section 3-4). The observabie decay gamma-ray lines from Blanket No. 2 are shown in Table 3-2. Analysis of the low energy Np -239 decay gamma-rays presents some difficulties as compared to the higher energy prompt gamma-rays, as will be explained subsequently. Other problems also arise.

The analysis of gamma-rays from the blanket is very different from the'conventional analysis of gammarays emitted by small samples. The neutron flux, hence the capture rate, inside the blanket varies precipitously with depth, as does the transmission probability of the escaping gamma-rays. Particularly when dealing with low energy gamma-rays the heterogeneity of the bianket composition is also a major consideration.

Comparison of Tables $3-1$ and 3-2 shows that sodium activity is prominent in both prompt and decay gammarays spectra. This enables one to inter-normalize the prompt and decay gamma-ray data and synthesize the 
TABLE 3-1

OBSERVABLE PROMPT GAMMA-RAYS FROM BLANKET NO.2

\begin{tabular}{|c|c|c|}
\hline Nuclei & Capture Line & Inelastic Iine \\
\hline$U-238$ & None Resolved & None Resolved \\
\hline $\mathrm{Fe}$ & $\begin{array}{c}\text { More than } 20 \text { prominent } \\
\text { peaks including } 7631.6 \mathrm{KeV} \\
\text { line }\end{array}$ & $845 \mathrm{KeV}$ \\
\hline $\mathrm{Na}$ & $\begin{array}{r}870.6 \mathrm{KeV} \\
6395.4 \mathrm{KeV}\end{array}$ & $438 \mathrm{KeV}$ \\
\hline $\mathrm{Cr}$ & $\begin{array}{r}835.1 \mathrm{KeV} \\
7939.3 \mathrm{KeV} \\
8884.1 \mathrm{KeV}\end{array}$ & $1433.9 \mathrm{KeV}$ \\
\hline 0 & None Resolved & $6127.8 \mathrm{KeV}$ \\
\hline $\mathrm{C}$ & $4945 . ? \mathrm{KeV}$ & None Resolved \\
\hline
\end{tabular}


Table 3-2

OBSERVABLE SHORT-LIVED DECAY GAMMA-RAYS

FR̊OM BLANKETT NOO. 2

\begin{tabular}{lccc}
\hline NUCLEI & ENERGY(KeV) & YIELD $(\%)^{*}$ & T I/2 \\
\hline \multirow{2}{*}{$\mathrm{Na}^{24}$} & 1368.7 & 100 & $15 \mathrm{hrs}$ \\
& 2753.9 & 102 & \\
$\mathrm{Cr}^{51}$ & 322 & & $27.8 \mathrm{~d}$ \\
$\mathrm{Fe}^{59}$ & 1286.6 & & $45.0 \mathrm{~d}$ \\
$\mathrm{~Np}^{239}$ & 106.1 & 22.8 & $2.3 \mathrm{~d}$ \\
& 209.8 & 4.1 & \\
& 228.2 & 12.7 & \\
& 277.6 & 1.1 & \\
& 315.9 & 2.5 & \\
& 334.3 & & \\
\hline
\end{tabular}

* YIELD defined here is the number of gammas per 100 disintegrations. The reference is

C. Lederer, J. Hollander, and I. Perlman

Table of Isotopes, 6th edition, John Wiley and Sons, Inc. 1967 
results into a single neutron balance. Although chromium and iron also appear in both spectra, they are not particularly suitable for this purpose due to the long half-life of $\mathrm{Cr}^{51}$ and $\mathrm{Fe}^{59}$, which results in accumulation of interfering residual activity over a long previous irradiation history.

3-2 Theory

The geometric configuration of the Blanket Test Facility is described in the preceding chapter, and in LMFBR Blanket Physics Project Progress Report No. I (Li). Figure 3-I shows the schematic overall plan view of the Blanket and the Ge(Li) detector set-up, where $\mathrm{x}_{\mathrm{B}}, \mathrm{x}_{\mathrm{R}}, \mathrm{x}_{\mathrm{F}}$ and $\mathrm{x}_{\mathrm{L}}$ are the thicknesses of blanket; reflector, LiF and lead, respectively, and $x_{A}$ is the distance from the outside surface of the blanket to that of the shielding door.

The number of prompt gamma-rays of interest with energy $\mathrm{E}_{\gamma}$ produced per second at the position $\mathrm{X}$ in the blanket is given by the following equation:

$$
C_{\gamma}(X)=\int_{E} N\left(\frac{I \gamma(E)}{100}\right) \sigma(E) \varnothing(E, X) d E,
$$

where

I $\gamma(E)$ - is the number of prompt gamma-rays with energy $\mathrm{E} \gamma$ emitted per 100 neutron reactions 


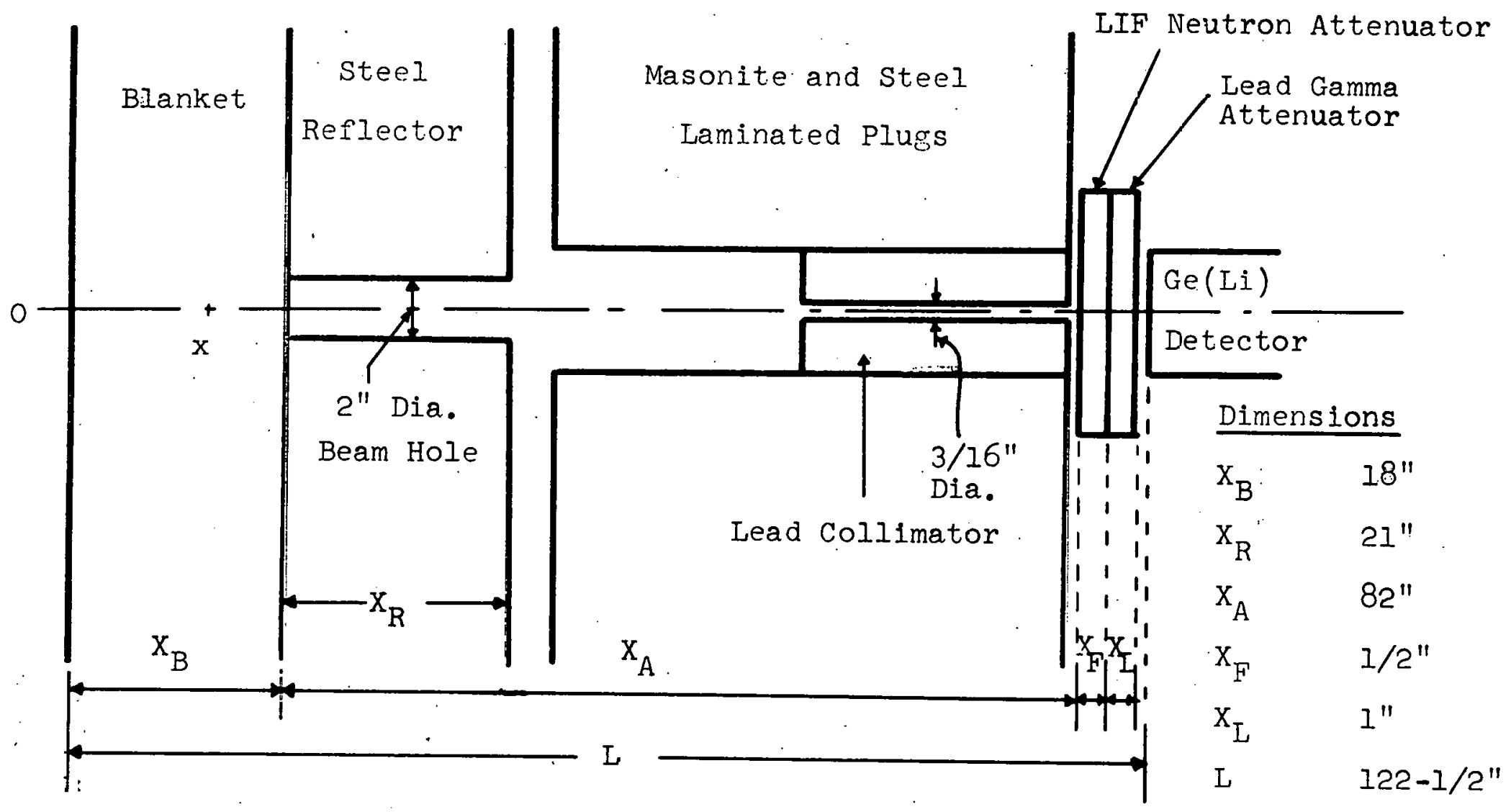

Fig. 3-1 Scinematic Overall Plan View of Blanket No. 2 and Detection Setup 
(capture or inelastic scattering) of energy $E$,

$N \quad$ - is the number of target nuclei of interest per c.c of the blanket (the medium is assumed to be completely homogeneous),

$\pi(E)$ - is the neutron cross-section for a certain reaction (capture or inelastic scattering) of the element of interest at the neutron energy $\mathrm{E}$, and

$\phi(E, X)$ - is the one-dimensional axial flux of neutrons having energy $E$ at the position $X$ in the blanket.

For a first step we may assume that the gamma-ray yield intensity function $I \gamma(E)$ is constant overall neutron energies. This may not be a very good approximation for certain constituents. However the efficiency curve for iron peaks shows that $I \gamma(E)$ does not vary appreciably with respect to the energy of the incoming neutrons. This will be discussed further in section 3-3-1.

Secondly the separability of variables for the neutron flux $\phi(E, X)$ is assumed as follows:

$$
\varnothing(E, X)=\varnothing(E) \psi(X)
$$


where $\phi(E)$ - is the neutron energy spectrum and

$$
\begin{aligned}
& \psi(\mathrm{X}) \text { - is the average one-group axial flux dis- } \\
& \text { tribution in the blanket, which is normalized } \\
& \text { as follows: } \\
& \int_{0}^{X_{B}} \psi(x) d X \equiv 1 .
\end{aligned}
$$

Calculations show that the collapsed one-group flux varies very smoothly with position. (Appendix A). Hence separability of the neutron flux $\phi(E, X)$ is quite an appropriate assumption for present purposes.

A homogeneous blanket medium and a steady state flux in the blanket is already assumed.

Hence, Equation 3-1 becomes

$$
C \gamma(X)=\left(\frac{I \gamma}{I 00}\right) \psi(X) \int_{E} N \sigma(E) \varnothing(E) d E
$$

Now define the average reaction rate over the neutron energy in the blanket, $R$, as follows:

$$
R \equiv \int_{E} N \sigma(E) \varnothing(E) d E
$$

This reaction rate, $R$, is classified into various kinds of reactions according to the specification of $N$ and $\sigma$. iv specifies the material of interest in the blanket and $\sigma$ specifies both the material and mode of reaction. 
The total area of the gamma-ray peak of interest with energy Er observed by the detector during the time interval, $\mathrm{T}$, is given by the following equation:

$$
A \gamma=\left(\int_{0}^{X_{B}} C \gamma(X) S \gamma(X) d X\right) \cdot T \cdot F \gamma \cdot E \gamma \cdot \Omega
$$

or

$$
A \gamma=R \cdot\left(\frac{I \gamma}{100}\right) \cdot S \gamma \cdot T \cdot E \gamma \cdot T \gamma \cdot \Omega
$$

where

$$
\mathrm{S} \gamma \equiv \int_{0}^{\mathrm{X}_{\mathrm{B}}} \Psi(\mathrm{X}) \mathrm{S} \gamma(\mathrm{X}) \mathrm{dX}
$$

$T$ - is the counting time,

$\mathrm{E} \gamma$ - is the intrinsic detection efficiency of the $\mathrm{Ge}(\mathrm{Li})$ detector and the associated electronics for gamma-ray energy $E \gamma$,

Fy - is the correction factor for attenuation of gamma-rays with energy $\mathrm{E} \gamma$ by air or any other materials placed in the flight path of the gamma-ray beam from the blanket surface to the detector,

$\Omega$ - is the geometric solid-angle correction factor, and

$S \gamma(X)$ - is the correction function for self-absorption of gamma-rays with energy $E \gamma$ in the blanket, which is a function of position. 
3-3 Prompt Capture Gamma-Rays

3-2-1 Total Efficiency

From Eq. 3-6 the total peak area at Ey observed by the detector may be rewritten as:

$$
A \gamma=\left(\frac{I \gamma}{I 00}\right) \cdot R \cdot E \gamma \cdot T
$$

where $\boldsymbol{\varepsilon} \gamma$ is the total efficiency which is defined by

$$
\boldsymbol{\varepsilon} \gamma \equiv \mathrm{S} \gamma \cdot \mathrm{F} \gamma \cdot \boldsymbol{\varepsilon} \gamma \cdot \Omega
$$

The total efficiency function, $\boldsymbol{\varepsilon}$, represents the contribution of the gamma-ray energy dependent terms and the constant terms to the reaction rate calculation. The function $\boldsymbol{\varepsilon} \gamma$ is computed by using an array $\boldsymbol{\varepsilon} j$ that stores all the terms for the $j$-th gamma-ray energy value, $E j$. The $\boldsymbol{E} j$ array values are determined by using the measured data on system efficiency, self-absorption and the variation of the neutron flux in the blanket, and the attenuation of the gamma-ray beam during the flight to the detector. The determination of the total efficiency curves to be used with the data involves consideration of complicated effects taking place during the flight from birth to detection. Thus the total efficiency of the setup has been determined experimentally instead of by caiculation. 
Since we are dealing for the most part with prompt gamma-rays of fairly high energy, a homogeneous medium was assumed and all heterogeneous effects on the selfabsorption correction factor are neglected. Since the same detector and geometry was used for all prompt gamma-ray experiments on Blanket No. 2, the same efficiency curve applies. The total efficiency array Ej that stores all the terms for $j$-th energy value is:

$$
\boldsymbol{\varepsilon}_{j}=\frac{A j}{\left(\frac{I j}{I 00}\right) \cdot R \cdot T},
$$

where $A j$ and $I j$ are the arrays of $A \gamma$ and $I \gamma$, respectively, for the $j$-th gamma-ray energy value.

Figure 3-2 shows the total efficiency curve for the detection system, which is obtained from the iron prompt capture gamma-ray data from Blanket No. 2, employing the yield intensity array $I j$ for thermal neutrons. Note that the observed total efficiency array data lie on the efficiency curve for thermal neutron capture (O1, H3, H4) except for a few of the peaks. The most prominent shift in yield occurs with the peak at $7278.9 \mathrm{KeV}$, which is considerably displaced from the thermal efficiency curve. This shows that some peaks do indeed change in yield intensities as the neutron energy 


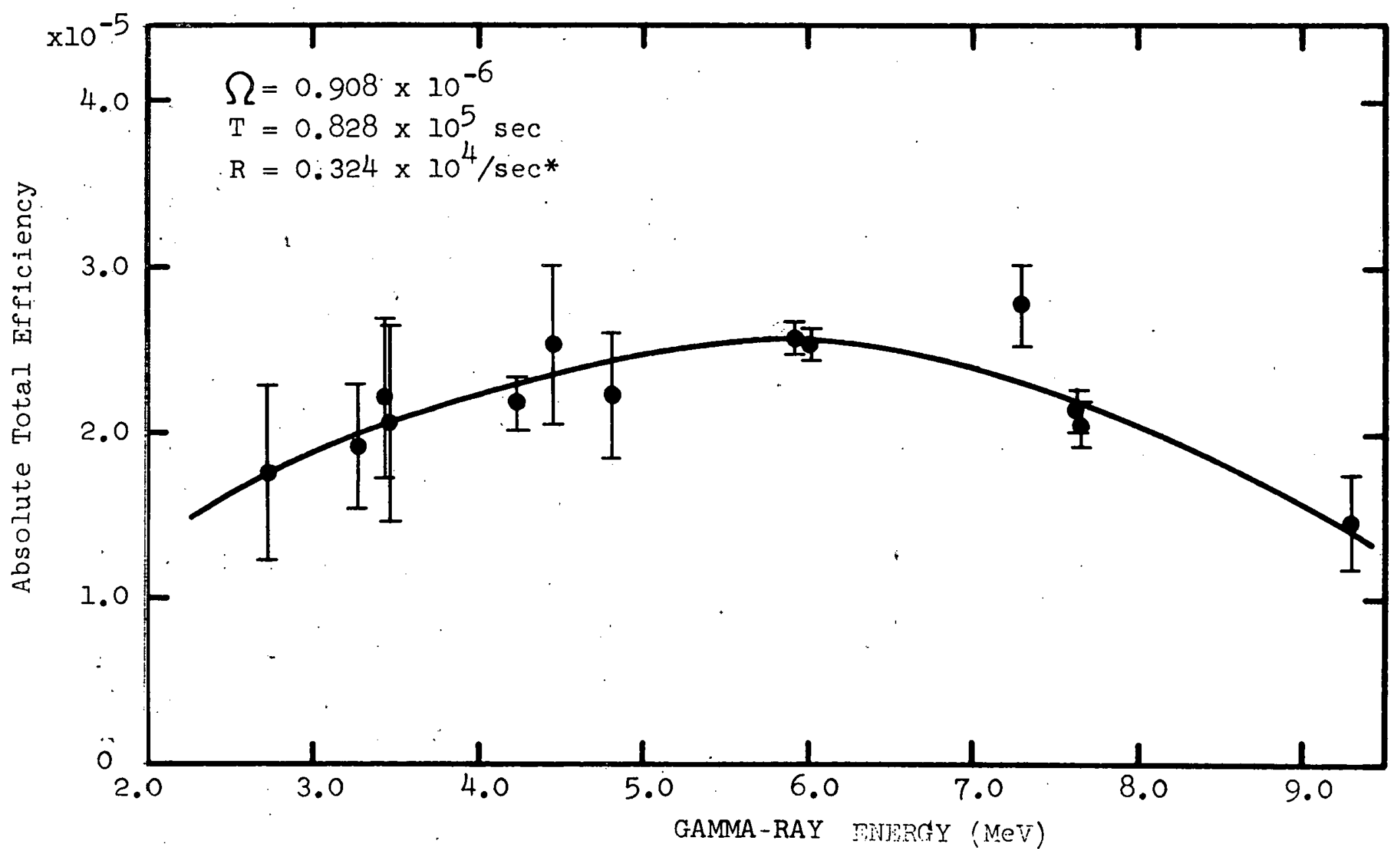

Fig. 3-2 Total Efficiency Curve of Detection System for High Gamma-Energies $\left(* \mathrm{R}=\mathrm{N} \bar{\sigma} \not \mathbf{D}: \mathrm{N}=0.01375, \bar{\sigma}=\mathrm{c} .00867, \bar{\phi}=2.72 \times 10^{8}\right)$ 
changes, while a substantial number of others do not. It is very important to know that there are some gammaray peaks which maintain an invariant yield regardless of the neistirn energy: Othcrwise dala Interpretation would be hopelessly complicated due to the paucity of prompt gamma-ray. yield data for all but thermal neutron energies.

Run number 105 is used to get the total efficiency data. Appendix I shows all GAMANL output data for the prompt and decay gamma-ray spectra from Blanket No. 2.

A background run has been performed with the 2-in. diameter beam hole in the reflector plugged to check background (especially $\mathrm{Fe}$ ). The counting rate was so small that no significant contribution from the reflector is observed. As the diameter of the collimators was decreased, the signal ratio of $\mathrm{Na}$ and $\mathrm{Cr}$ prompt capture lines to Fe prompt capture lines improved a great deal. 3-3-2 Data Analysis

Table 3-3 shows the major background lines observed in spectra from Blanket No. 2. Among these background peaks Ar-41 at $1293.6 \mathrm{KeV}$, was always so prominent that it was used as an energy calibration, together with the 
Table 3-3

PRINCIPAL BACKGROUND GAMMA-RAYS

\begin{tabular}{lc}
\hline ENERGY (KeV) & REMARKS \\
\hline 476.3 & B-12 Decay \\
511.0 & Anninilation \\
595.0 & Ge-74 Inelastic \\
691.4 & Ge-72 Internal \\
1173.2 & Conversion \\
1293.6 & Ar-41 Decay \\
1332.6 & Co-60 Decay \\
1460.5 & K-40 Decay or \\
1778.9 & Ar-40 Inelastic \\
4945.2 & C Capture, Prompt Gamma \\
7367.7 & Pb Capture, Prompt Gamma \\
7723.8 & Al Capture, Prompt Gamma \\
\hline
\end{tabular}


annihilation peak at $511 \mathrm{KeV}$. There are also two interesting background peaks at $595 \mathrm{KeV}$ and $691.4 \mathrm{KeV}$. They are, respectively, an inelastic scattering gamma line and the internal conversion line of Ge caused by interaction of incident fast neutrons with the Ge(Li) detector itself. The line at $691.4 \mathrm{KeV}$ is analyzed in considerable detail in Chapter $V$, since it permits inference of the fast neutron spectrum. The prompt capture line of carbon at $4945.2 \mathrm{KeV}$ is observed, originating perhaps in the masonite $12 \mathrm{CHI}$ port shield or even in the distant converter assembly itself; the other lines are general background lines which are all possible emissions from the structual materials around the facility.

Table 3-2 showed the prompt capture gamma-rays of $\mathrm{Fe}, \mathrm{Na}$, and $\mathrm{Cr}$ which were observable in the spectra of Blanket No. 2. Iron always showed more than 20 peaks in every spectrum obtained from Blanket No. 2. It should be noted that the shielding for the facility is magnetite concrete and the blanket reflector is pure iron. Therefore one can expect iron prompt capture lines from almost everywhere. In this case, if one is interested in the iron peaks due only to the blanket, a judicious choice of collimator design and careful alignment of 
the beam holes between the blanket assembly and the two movable shielding doors is called for. A specially built 2 -ft. long and $3 / 16$ in. internal diameter collimator was used in order to view only the bottom of the exposed blanket surface at the beam hole. This collimator eliminated most of the background iron lines from the side wall of the iron reflector. Sodium showed two prompt capture gamma-ray peaks at $870.6 \mathrm{KeV}$ and 6395.4 $\mathrm{KeV}$, and two major decay gamma-ray lines at $1368.7 \mathrm{KeV}$ and $2754.2 \mathrm{KeV}$. These two decay gamma-rays made it possible to relate the prompt capture gamma-rays and the decay gamma-rays, and to deduce the reaction rate of $\mathrm{U}-238$, as is discussed in section 3-4. Chromium showed three prompt capture gamma-rays at 835.1-, 7939.3-, and $8884.1-\mathrm{KeV}$.

The neutron capture reaction rate calculation for iron in Blanket No. 2 is shown in Table 3-4, and that for $\mathrm{Na}$, and $\mathrm{Cr}$ is shown in Table 3-5. Two runs, No. 104 and 105, are compared in both tables. GAMANL output data for runs, No. 104 and 105, are presented in Appendix I. Two different sized collimators, 5/16 in. dia. and 3/16 in. dia., were used in runs No. 104 and 105, respectively. Run number 105 in Table 3-4 shows that the reaction rate, $R$, lies within a $\pm 6 \%$ error except for the 


$$
\text { TABLE } \quad 3-4
$$

REACTION. RATE CALCULATIONS FOR IRON IN BLANKET NO. 2

RUNS NO. 104 and 105

\begin{tabular}{|c|c|c|c|c|c|c|}
\hline \multirow[t]{2}{*}{ ENERGY $(\mathrm{KeV})$} & \multirow[t]{2}{*}{ YIELD $(\%)$} & EFFICIENCY $\left(10^{-4}\right)$ & \multicolumn{2}{|c|}{$\begin{array}{cc} & \text { RELATIVE } \\
\text { AREA } & \text { REACTION RATE }\end{array}$} & \multicolumn{2}{|c|}{$\begin{array}{cc} & \text { RELATIVE } \\
\text { AREA } & \text { REACTION RATE }\end{array}$} \\
\hline & & & & un No: 104 & & un No. 105 \\
\hline 1613.0 & 5.85 & 0.665 & $\cdots$ & & 1014.9 & 3.23 \\
\hline 4218.8 & 4.02 & 0.235 & 553.6 & 2.74 & 2446.8 & 3.13 \\
\hline 5920.5 & 8.29 & 0.254 & 2203.9 & 4.90 & 5694.1 & 3.26 \\
\hline 6018.5 & 8.08 & 0.254 & 1598.1 & 3.64 & 5443.3 & 3.20 \\
\hline 7278.9 & 4.60 & 0.222 & 716.8 & 3.28 & 3383.7 & 4.00 \\
\hline 7631.6 & 27.19 & 0.211 & 4073.3 & 3.32 & 15793.5 & 3.32 \\
\hline 7645.6 & 22.14 & 0.211 & 3100.3 & 3.10 & 12244.9 & 3.16 \\
\hline
\end{tabular}


Table $3-5$

REACTION RATE CALCIIATIONS FOR Na AND Cr

IN BLANKET NO.2, RUNS NO. 104 AND 105

\begin{tabular}{lccrrrr}
\hline & RUN NUMBER & \multicolumn{2}{c}{104} & \multicolumn{2}{c}{105} \\
\hline ENERGY $(\mathrm{KeV})$ & YIELD(\%) & EFF. $\left(10^{-4}\right)$ & AREA & R & AREA & R \\
\hline $870.6(\mathrm{Na})$ & 25.39 & $0.908^{*}$ & 3220.4 & 0.590 & 5838.0 & 0.305 \\
$6395.4(\mathrm{Na})$ & 25.69 & 0.247 & 330.2 & 0.243 & 1533.4 & 0.292 \\
$835.1(\mathrm{Cr})$ & 24.04 & $0.937^{*}$ & 3385.1 & 0.608 & 6778.7 & 0.363 \\
$7939.3(\mathrm{Cr})$ & 11.41 & 0.200 & -- & -- & 446.2 & 0.236 \\
$8884.1(\mathrm{Cr})$ & 24.14 & 0.163 & 202.9 & 0.241 & 1238.0 & 0.380 \\
\hline
\end{tabular}

* Total Efficiency data for low energy gamma-rays are calculated in Section 3-5:2 
peak at $7278.9 \mathrm{KeV}$, which indicates that good collimation is very important in this experiment. This also confirms the accuracy of the measured reaction rates.

As mentioned previously, the calculation is based. upon the key assumption that the yield intensity does not change with neutron energy. In Section 3-5-2 we show that certain peaks from sodium and chromium do not change in yield intensity. The calculation of the capture reaction of $\mathrm{U}-238$ in $\mathrm{Blanket}$ No. 2 is discussed in the next section. This is done by analyzing the low energy short-lived decay gamma-rays from $\mathrm{Np}-239$. 3-4 Decay Gamma-Rays

3-4-1 Induced Radioactivity in the Blanket

Consider a neutron capture reaction at the position $\underline{r}$ in the blanket, at time $t$, which will produce a radioactive nuclide $A$ which decays into a stable nuclide $B$. The rate of decay, $d A / d t$, of a nuclide $A$ is given by the following equation:

$$
\frac{d A(\underline{r}, t)}{d t}=\int_{E} N(\underline{r}) \sigma_{C}(E) \phi(E, \underline{r}) d E-A(\underline{r}, t) \lambda_{A},
$$

where $\lambda_{A}$ is the radioactive decay constant of nuclide $A$, and the remaining terms are the same as previously described. Now the separability of variables for the 
50

neutron flux is again assumed and Eq. 3-1l can be written

$$
\frac{d A(\underline{r}, t)}{d t}=R \cdot n(\underline{r}) \cdot \psi(\underline{r})-A(\underline{r}, t) \lambda_{A},
$$

where

$$
\begin{aligned}
& n(\underline{r})=N(\underline{r}) / N \text {; the heterogeneity function, } \\
& R=\int_{E} N \sigma_{C}(E) \varnothing(E) d E,
\end{aligned}
$$

is defined as the capture rate, and $N$ is the homogenized concentration. After the irradiation for the time interval $t_{1}$, the number of radioactive nuclei, $A$, at the position $\underline{r}$ in the blanket, at time $t$ is therefore:

$$
A(\underline{r}, t)=\frac{R \psi(\underline{r}) n(\underline{r})}{\lambda_{A}}\left(e^{-\lambda_{A} t}-e^{-\lambda_{A}\left(t_{1}+t\right)}\right) \cdot(3-13)
$$

In a number of cases a radioactive nuclide $A$, which is produced by a neutron capture reaction decays into a nuclide $B$ which is also radioactive. In this case the number of radioactive nuclei, $B$, at the position $\underline{r}$ in the blanket, at time $t$ after an irradiation for the time interval $t_{1}$ is:

$$
\begin{aligned}
B(\underline{r}, t) & =\frac{R \psi(\underline{r}) n(\underline{r})}{\lambda_{B}-\lambda_{A}}\left(e^{-\lambda_{A} t}-e^{-\lambda_{A}\left(t+t_{I}\right)}\right. \\
& \left.\therefore \frac{\lambda_{A}}{\lambda_{B}} e^{-\lambda_{B}\left(t+t_{I}\right)}-\frac{\lambda_{A}}{\lambda_{B}} e^{-\lambda_{B} t}\right),
\end{aligned}
$$


where $\lambda_{A}$ and $\lambda_{B}$ are the radioactive decay constants of nuclides $A$ and $B$, respectively.

$\mathrm{Na}^{23}$ is in the former group of radioactive nuclides and $\mathrm{Np}-239$ is in the latter group. Hence, Equations 3-13 and 3-14 can be thought of as giving the number of the radio-active nuclei of interest produced by a neutron capture reaction of sodium and $U-238$, respectively.

Now the number of decay gamma-rays of interest with energy. E $\gamma$ produced at the position $\underline{r}$ in the blanket, at time $t$ is given by the following equation:

$$
\operatorname{cr}(\underline{r}, t)=\left(\frac{I \gamma}{100}\right) \lambda_{\eta} \eta(\underline{r}, t)
$$

where I $\gamma$ - is the number of decay gamma-rays with energy E $\gamma$ emitted per 100 disintegrations of the nuclide of interest. Hence, the total area under the decay gamma-ray peak of interest observed by the detector at energy Ey during the time interval between $t_{s}$ and $t_{f}$ is given by the following equation:

$$
A \gamma=\left(\int_{t_{S}}^{t_{f}} d t \int_{\Delta V} d V C \gamma(\underline{r}, t) S \gamma(\underline{r})\right) \cdot F \gamma \cdot \epsilon_{\gamma} \cdot \Omega,(3-16)
$$

where $\Delta V$ is the volume of blanket which can be seen by the detector through the beam hole. Substituting Eq. 3-15 into Eq. 3-16, the total area is:

$$
\begin{aligned}
A \gamma= & \left(\frac{I \gamma}{I 00}\right) \cdot \lambda_{\eta} \cdot\left(\int_{t}^{t} f t \int_{\Delta V} d V \eta(\underline{r}, t) s \gamma(\underline{r})\right) \\
& \cdot F \gamma \cdot \epsilon \gamma \cdot \Omega .
\end{aligned}
$$


According to decay modes, $\eta(\underline{r}, t)$ may be Equation 3-13 or Equation 3-14. However Equation 3-17 will be:

$$
A \gamma=\left(\frac{I \gamma}{I 00}\right) \cdot R \cdot T \cdot S \gamma \cdot F \gamma \cdot E \gamma \cdot \Omega \text {, }
$$

where

$$
\begin{aligned}
T_{N a}= & \int_{t_{S}}^{t_{f}}\left(e^{-\lambda_{N a} t}-e^{-\lambda_{N a}\left(t_{I}+t\right)}\right) d t ; \\
T_{N p}= & \int_{t_{S}}^{t_{f}} \frac{\lambda_{N p}}{\lambda_{N p}-\lambda_{U}}\left(e^{-\lambda_{U} t}-e^{-\lambda_{U}}\left(t+t_{I}\right)+\frac{\lambda_{U}}{\lambda_{N p}}\right. \\
& \left.e^{-\lambda_{N_{p}}\left(t \mid t_{I}\right)}-\frac{\lambda_{U}}{\lambda_{N p}} e^{-\lambda_{N p} t}\right) d t \\
S \gamma^{N a} & =\int_{\Delta V} d V \psi(\underline{r}) n_{N a}(\underline{r}) s \gamma^{N a}(\underline{r})
\end{aligned}
$$

and

$$
S \gamma^{\mathrm{U}}=\int_{\Delta \mathrm{V}} \mathrm{dV} \psi(\underline{\mathrm{r}}) \mathrm{n}_{\mathrm{U}}(\underline{\underline{r}}) \mathrm{S} \gamma^{\mathrm{U}}(\underline{\underline{r}}) .
$$

All terms will be discussed further, one-by-one in the two following sections. 
3-4-2 Correction Factors

From Equation $3-18$ one can see that four factors are involved to account for all processes anticipated from the birth of the gamma-rays to their detection by the Ge(Li) detector. In the previous case of the high energy gamma-ray analysis, we combined all these four factors into one factor, the so-called total efficiency. But when one deals with low energy gamma rays the heterogeneity effect does not allow combination of all factors into a single factor since the selfabsorption correction factor will be very different according to where the gamma-rays originate. In this section two correction factors, $F \gamma$ and $S \gamma$, are discussed and the intrinsic efficiency, $\epsilon_{\gamma}$, and the solid angle factor, $\Omega$, will be discussed in the next section.

$F \gamma$ is the attenuation correction factor for gamma-rays of energy Ey by air, LiF, lead, or any other materials placed in the flight path of the gamma-ray beam from the blanket surface to the detector. If we assume that there are air, LiF and lead, thickness of $\mathrm{X}_{A}, \mathrm{X}_{F}$, and $\mathrm{X}_{L}$, respectively 
between the surface of the blanket and the Ge(Li) detector surface (ref. Fig. 3-1), the attenuation correction factor is given by

$$
\left.F \gamma=e^{-\left(\mu_{\gamma}^{A}\right.} X_{A}+\mu_{\gamma}^{F} X_{F}+\mu_{\gamma}^{L} X_{L}\right)
$$

where $\mu_{y}^{\mathrm{A}}, \mu_{\gamma}^{\mathrm{F}}$, and $\mu_{\gamma}^{\mathrm{L}}$ are the total linear attenuation coefficients of air, LiF, and lead, respectively, for gamma-rays of energy E $\gamma$.

The treatment of the self-absorption factor is somewhat different than that of ordinary small samples, : since the gamma-ray emitting "sample" is as large as the blanket itself. Furthermore, when dealing with decay gamma-rays the heterogeneity effect of the blanket can no longer be ignored, since the energy of the gammarays is typically much lower than that of prompt gammarays, and in the range where gamma-ray attenuation coefficients are very large. The self-absorption correction factor is defined as the ratio of the total number of gamma-rays of interest with energy $\mathrm{E} \gamma$ appearing at the outside surface of the blanket per second to the total number of gamma-rays of interest with the same 
energy Er produced throughout the blanket per second. This factor is dependent on the thickness, the composition, the structural order of the blanket, the neutron flux shape in the blanket, and the energy of the gamma-rays of interest. Therefore the self-absorption correction factor is completely different according to where the decay gamma-rays originate, $\mathrm{U}$-fuel rods or $\mathrm{Na}_{2} \mathrm{CrO}_{4}$ filling. Calculations of self-absorption correction factors for gamma-rays from both $\mathrm{U}$-fuel rods and $\mathrm{Na}_{2} \mathrm{CrO}_{4}$ filling are done in Appendix E. Computer codes SELFN and SELFU are also presented in Appendix E. The computer programs perform the double integration of Equations $3-21$ and 3-22, The calculated values are compared with the measured values of the attenuation coefficients in a fuel rod lattice in SELFU. Figure 3-3 shows the calculated self-absorption correction factors for gamma-rays from $\mathrm{Na}_{2} \mathrm{CrO}_{4}$ by SELFN. Figure 3-4 shows the calculated (circular dots) and measured (triangular dots) selfabsorption correction factors for gamma-rays from U-fuel rods of Blanket No. 2 by SELFU. Gamma-ray linear attenuation coefficients of $\mathrm{Na}_{2} \mathrm{CrO}_{4}$ and Uranium were independently obtained by a series of measurements (Appendix B). A test box was prepared to measure the actual attenuation factor of the fuel rod cell. Obviously all curves of Figures 3-3 and 


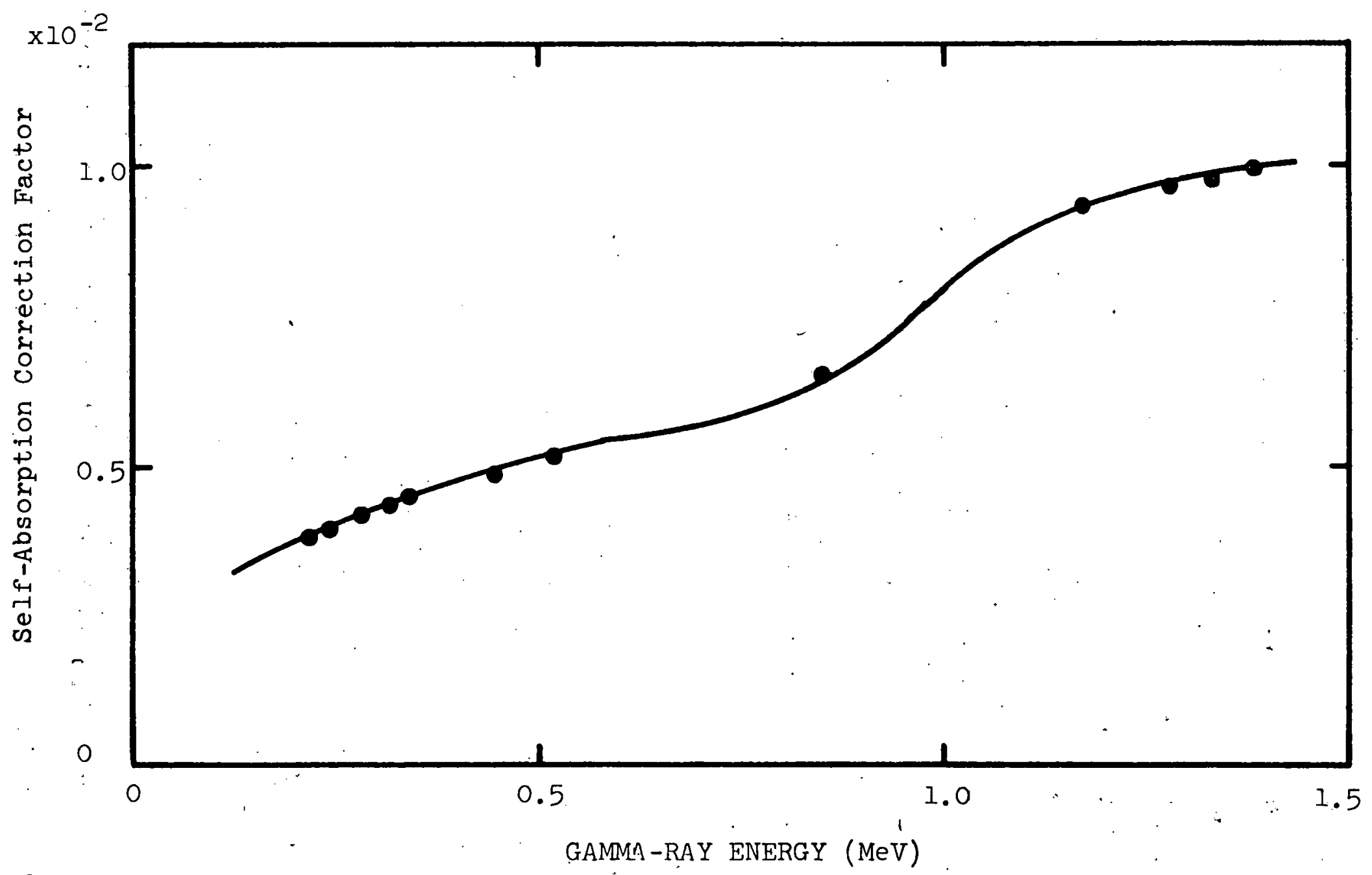

Fig. 3-3 Self-Absorption Correction Factors for Gamma-Rays from $\mathrm{Na}_{2} \mathrm{CrO}_{4}$ of Blanket No. 2 (calculated by SELFN) 


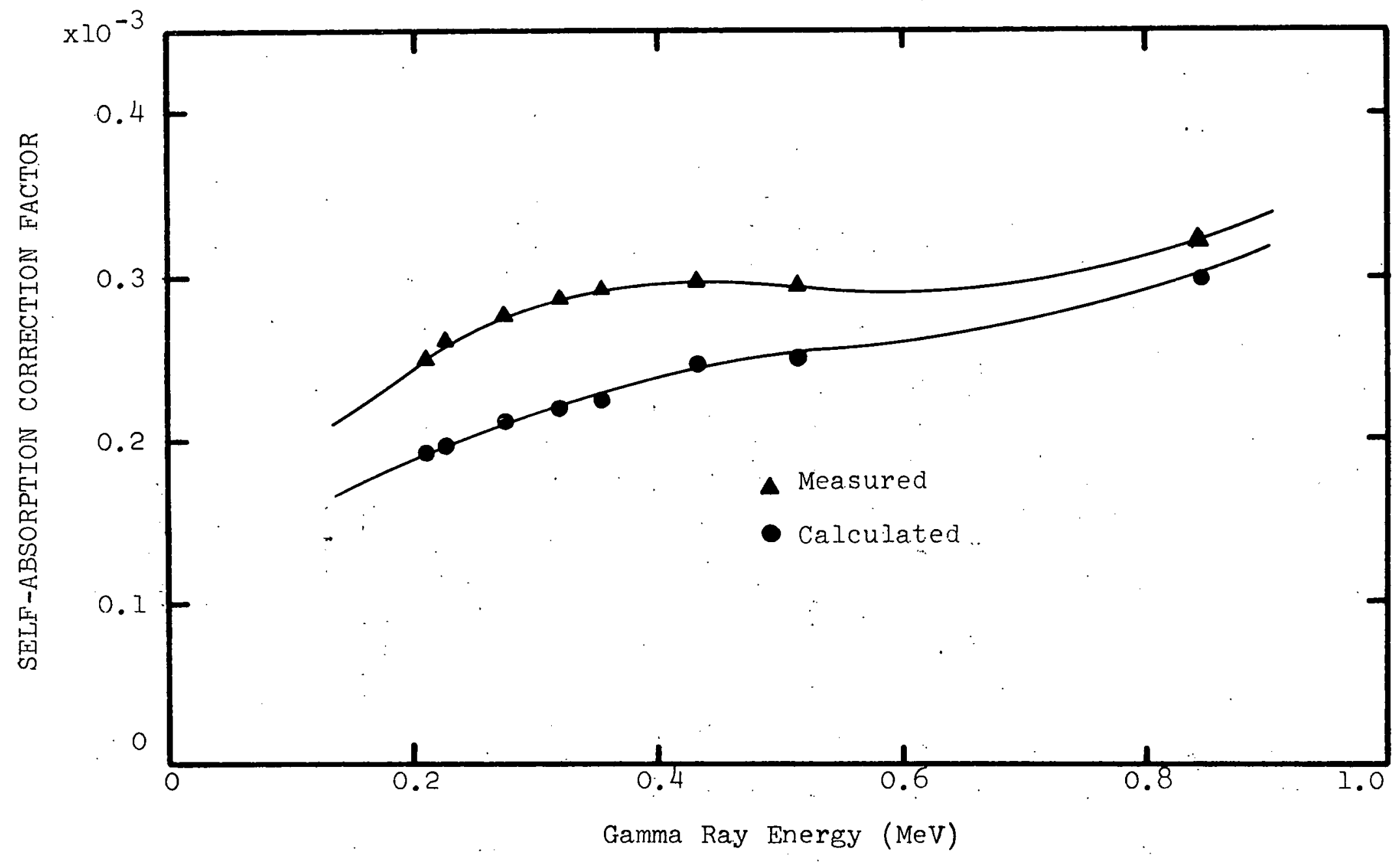

Fig. 3-4 Self-Absorption Correction Factor for. Gamma-Rays from U-Fuel Rods of Blanket No. 2 (Calculated by SELFU) 
3-4 ought to converge to the same value, since the heterogeneous effect decreases. as the gamma-ray energy. increases. When the calculation of the self-absorption factor for gamma-rays of $\mathrm{Np}-239$ is carried out, neither possible structural disorder nor scattered gamma-rays were considered. Furthermore the uncertainty increases as the energy of the gamma-rays decreases, because of the high linear attenuation coefficients. Calculation shows that most of the Np-239 gamma-ray contributioncomes from the first row of fuel rods, but the actual measurement showed that the contributions from other rows of fuel rods were not negligible. This indicates that small changes in fuel pin structure would produce a considerable change in the calculated self-absorption factors.

The intrinsic efficiency data can be obtained by a series of experiments without major difficulty. This will be discussed in the next section. 3-4-3 Intrinsic Efficiency

The intrinsic efficiency factor is the number of counts recorded at the energy $\mathrm{E} \gamma$ in the multi-channel analyzer per gamma-ray arriving at the surface of the Ge(Li) detector. Figure 3-5 shows the schematic setup for the efficiency measurements. An adjustable callbrated 


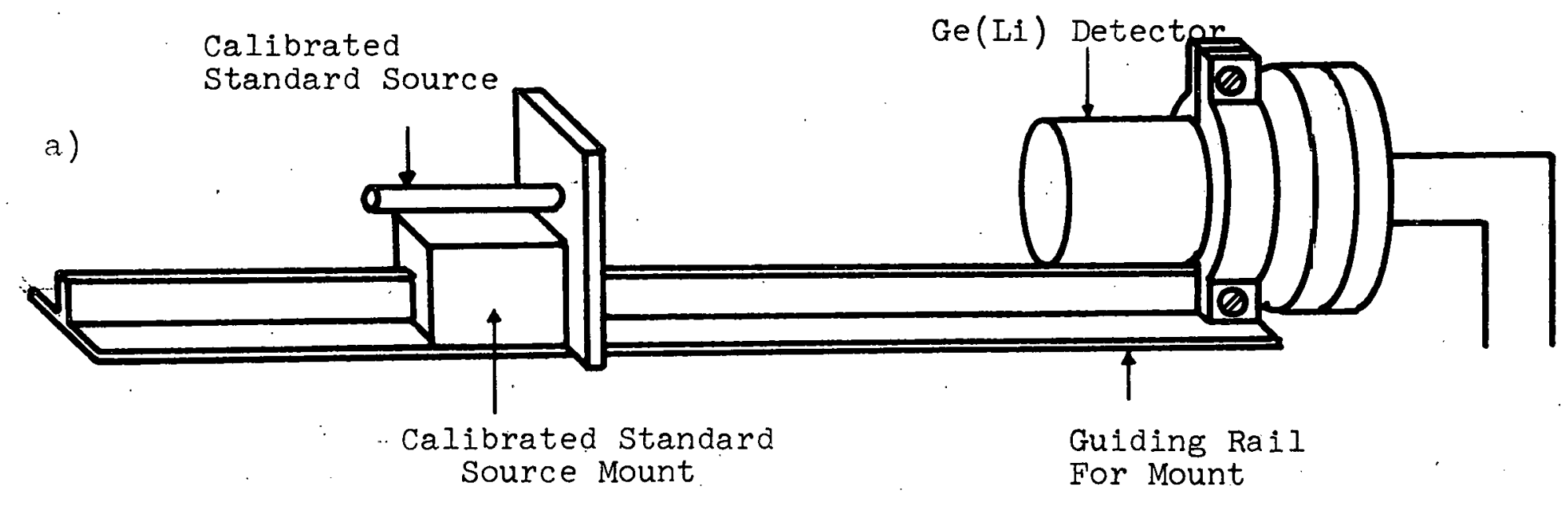

b) Plan View

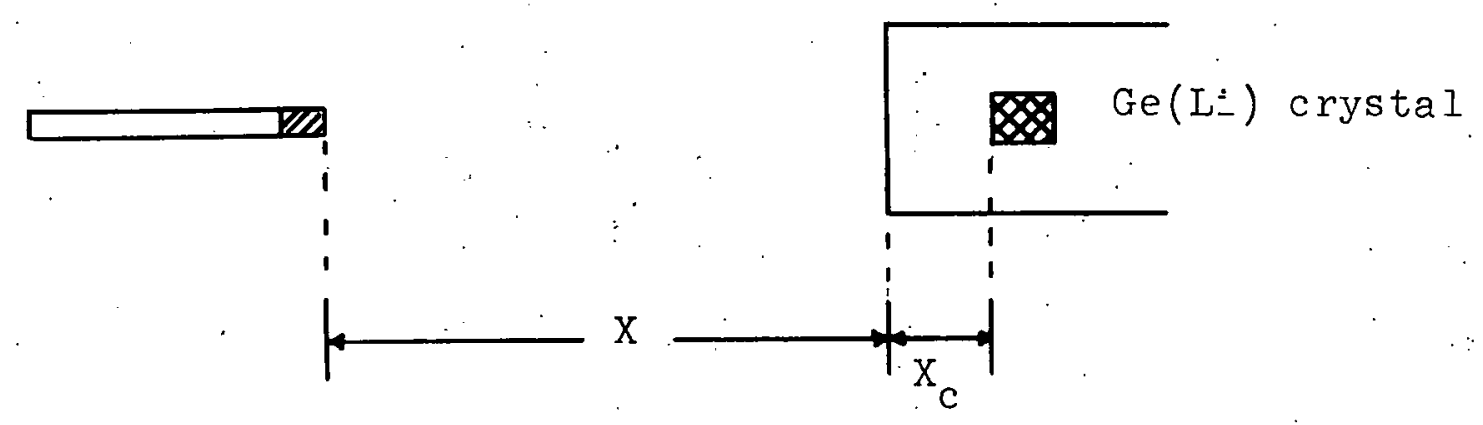

Fig. $3-5$

Schematic View of the Setup for the Efficiency Mëasurements 
source mounting system made of aluminum is used with the 17 c.c. crystal detector so it could be mounted with fixed reference to the crystal.

The intrinsic efficiency of the detection system including the solid-angle correction factor, $\boldsymbol{\varepsilon}=\boldsymbol{\epsilon} \boldsymbol{\Omega}$, may be written as follows:

$$
\boldsymbol{E}=\mathrm{A} \gamma / \mathrm{AO} \cdot \mathrm{Cd} \cdot\left(\frac{\mathrm{I} \gamma}{\mathrm{IOO}}\right) \cdot \mathrm{F} \gamma \cdot \mathrm{T}
$$

where

$$
\begin{aligned}
& \text { Ao - is the initial activity from the calibrated } \\
& \text { standard source, } \\
& \text { Cd - is the decay correction factor for the time } \\
& \text { of initial calibration to the time of the } \\
& \text { experiment. }
\end{aligned}
$$

The geometric component of the intrinsic efficiency can be exactly expressed by the following equation since the effective distance from the source to the detector depends on the size of the crystal and the distance from the aluminum wall to the crystal (ref. Fig. 3.5(b)).

$$
\begin{gathered}
\boldsymbol{\epsilon}=\frac{\mathrm{C}}{\left(\mathrm{X}+\mathrm{X}_{\mathrm{C}}\right)^{2},} \\
\text { or } \boldsymbol{\epsilon}^{-1 / 2}=A_{0}+A_{1} \mathrm{X},
\end{gathered}
$$


where

C, $A_{0}$, and $A_{1}$ - are constants with respect to the distance,

$X=$ is the distance betweon the source and the surface of the detector, and

$x_{c}$ - is the distance between the crystal and the surface of the detector face.

Equation 3-26 is a. Iinear equation with respect to the distance, $x$, where $A_{0}$ and $A_{1}$ are functions of the gamma-ray energy.

Efficiency runs were made using a series of calibrated sources purchased from the New England Nuclear Corporation. Table 3-6 shows the information supplied by the manufacturer for the sources which were used in the measurement. A dozen runs were made at various distances from the detector with each source. Figure 3-6 shows the resultant inverse of the square root of the efficiency versus the distance from the surface of the detector. AII slopes and intercepts were obtained using a linear least square fit with very satisfactory results. A computer program INTEF calculate the intrinsic efficiency of the system (Appendix D). Using the computer program INTEF the detector efficiency of the 17 c.c. Ge(Li) crystal was obtained and plotted in 
Table $3-6$

\section{STANDARD SOURCES}

(NEW ENGLAND NUCLEAR CORPORATION)

\begin{tabular}{|c|c|c|c|c|c|}
\hline ISOTOPE & $\begin{array}{l}\text { INTTIAL } \\
\text { ACTIVITY } \\
(\mu \mathrm{Ci})\end{array}$ & $\begin{array}{c}\text { CALIBRATION } \\
\text { DATE }\end{array}$ & HALF-LIFE & $\begin{array}{l}\text { ENERGY* } \\
(\mathrm{KeV})\end{array}$ & $\begin{array}{l}\text { YIELD** } \\
(\%)\end{array}$ \\
\hline $\mathrm{Co}^{57}$ & 10.7 & $3-1-69$ & $272 d$ & $\begin{array}{l}122.05 \pm 0.05 \\
136.40 \pm 0.06\end{array}$ & $\begin{array}{l}87 \\
10.5\end{array}$ \\
\hline $\mathrm{Cs}^{137}$ & 12.4 & $9-2-69$ & $30 \mathrm{y}$ & $661.595 \pm 0.076$ & 85 \\
\hline $\mathrm{Mn}^{54}$ & 10.5 & $3-1-69$ & $314 \mathrm{~d}$ & $834.9 \pm 1.1$ & 100 \\
\hline $\mathrm{Co}^{60}$ & $\because 7.5$ & $9-2-69$ & $5.26 \mathrm{y}$ & $\begin{array}{l}1173.226+0.040 \\
1332.48 \mp 0.05\end{array}$ & $\begin{array}{l}99 \\
99\end{array}$ \\
\hline $\mathrm{Na}^{22}$ & 9.4 & $3-1-69$ & $2.60 \mathrm{y}$ & $\begin{array}{l}511.006+0.005 \\
1275.0 \quad \mp 0.8\end{array}$ & $\begin{array}{r}180 \\
99\end{array}$ \\
\hline
\end{tabular}

* References are on Table 2-2

** C.: Lederer et al., "Table of. Isotopes" 6th Ed.,. John Wiley and Sons, Inc. 


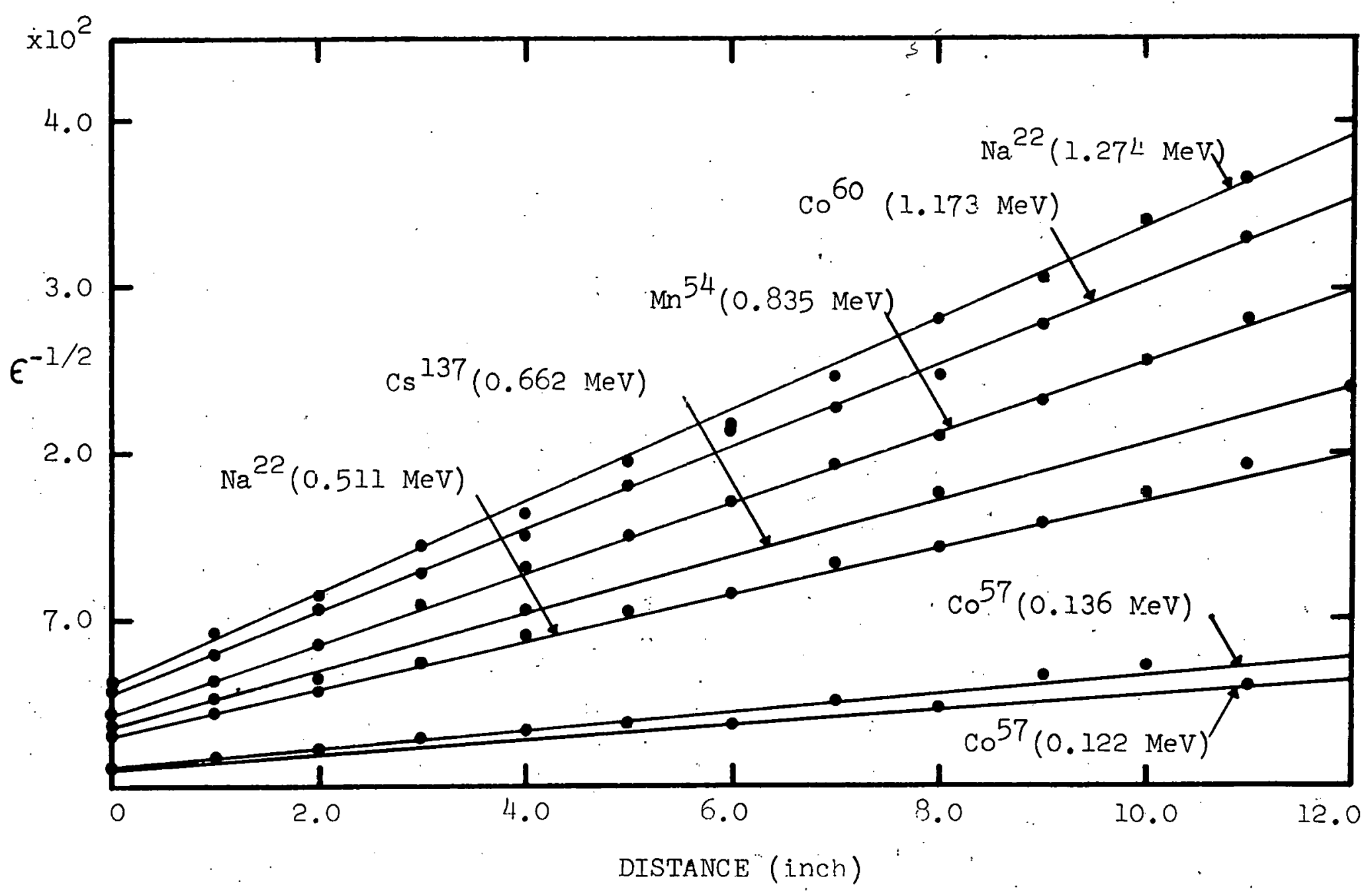

Fig. 3-6 Variation of Intrinsic E:ficiency with Distance from Detector 
Figure 3-7. The triangular points in Figure 3-7 are the data reported by odette (02) for the same detector and the same sources. The good agreement between the two calculations demonstrates excellent consistency in the efficiency measurements. It would have been desirable to have more standard sources especially in the range between $136 \mathrm{KeV}$ and $511 \mathrm{KeV}$. However interpolation should be adequate in this region. The estimated error in this experiment is on the order of $5-10 \%$, most of which is attributable to the uncertainty in the initial activity of the sources.

The measurement of intrinsic efficiency by this method is much more accurate and reliable than that using a single fixed position for the gamma-ray sources. In this method one can not only have better statistics than any other method, but also correct the efficiency for the effective distance between the gamma-ray source and the detector. 3-4-4 Capture Reactions in $\mathrm{Na}$ and $\mathrm{U}-238$

Two radioactive is otopes, $\mathrm{Na}-24$ and $\mathrm{Np}-239$, are of primary interest in the analysis of the blanke $t$ decay gamma-ray spectra. The Na-24 decay gamma-ray lines at $1368 \mathrm{KeV}$ and $2754 \mathrm{KeV}$ are used to internormalize the prompt capture gamma-rays and the decay gamma-rays in 


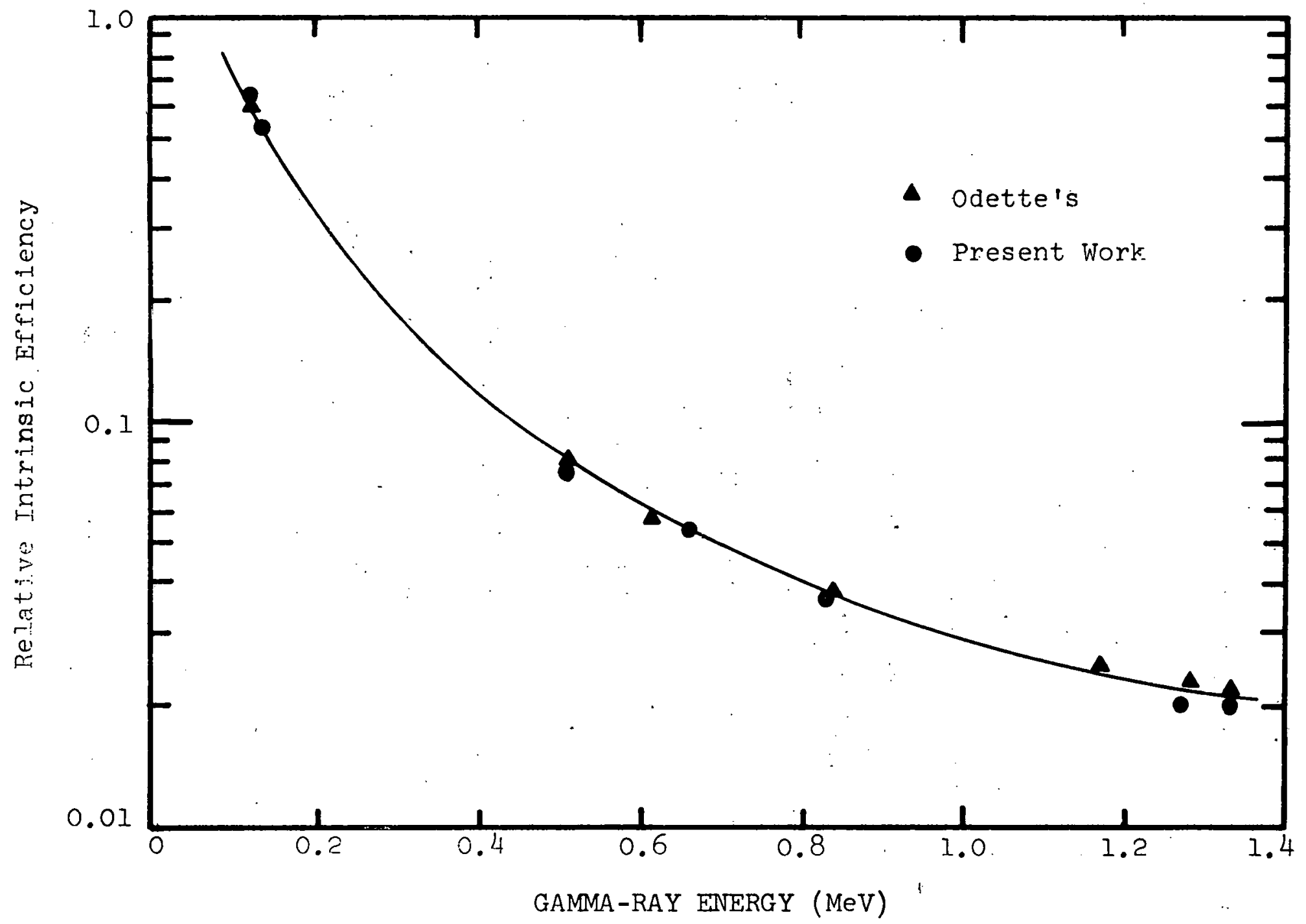

Fig. 3-7 Intrinsic Efficiency of 17 c.c. Ge(Li) Detector 
the analysis of the neutron capture reactions. Since the capture reaction rate of sodium in the blanket is already known through analysis of the prompt capture gamma-rays, the neutron capture rate of $\mathrm{U}-238$ can be obtained by analyzing the decay gamma-rays from Np-239 along with the decay gamma-rays of the sodium. When a neutron is captured by a $\mathrm{Na}^{23}$ nucleus, $\mathrm{Na}^{24}$ is formed, which will decay into a stable nuclide, $\mathrm{Mg}^{24}$, which a half-life of $15 \mathrm{hrs}$. This radioactive decay is accompanied by the emission of two prominent gamma-rays. The neutron capture rate of sodium, therefore, can be obtained by analyzing the gamma-rays from $\mathrm{Na}^{24}$ decay.

$\mathrm{Np}-239$ comes from the $\beta$-decay of $\mathrm{U}-239$, which is formed by the neutron capture reaction of $U-238$.

$$
\mathrm{U}-238+o^{\mathrm{n}^{1}}-\mathrm{U}-239 \frac{\beta}{23.5 \mathrm{~m}} \mathrm{~Np}-2392 \frac{\beta}{2.35 \mathrm{~d}} \mathrm{Pu}-239 \quad(3-27)
$$

$\mathrm{Np}-239$ is radioactive itself and decays into $\mathrm{Pu}-239$ by $\beta$-decay, coincidentwith the emission of more than 30 gamma-ray peaks (L2). The following peaks are the most prominent.

\begin{tabular}{lrrrrrr}
\hline $\mathrm{E}_{\mathrm{O}}(\mathrm{KeV})$ & 106.14 & 209.8 & 228.2 & 277.6 & 315.9 & 334.3 \\
$\mathrm{I \gamma}(\%)$ & 22.8 & 4.1 & 12.7 & 14.1 & 1.5 & 2.0 \\
\hline
\end{tabular}


These peaks were all observed in the short-lived decay gamma-ray spectra of blanket No. 2(see Runs No. 99, 102, and 103 in Appendix I). Table 3-7 shows the capture reaction rates of $\mathrm{U}-238$ and $\mathrm{Na}$ deduced from analysis of decay gamma-rays from $\mathrm{Np}-239$ and $\mathrm{Na}-24$, respectively. Two different successive runs, No. 102 and 103, were analyzed for various gamma-rays from $\mathrm{Np}-239$. Both runs are analyzed using the computer program GAMANI and plotted in Appendix I. Table 3-7 shows that run-to-run reproducibility is good, but that the resultant capture rate inferred tends to increase with increasing gamma-ray energy. This difference results mainly from the difference between the calculated and real self-absorption correction factor. 3-5 Inelastic Scattering Gamma-Rays. 3-5-1 Inelastic Scattering Rates other observable gamma-rays in the prompt gamma-ray spectra from Blanket No. 2 are inelastic gamma-rays. Inelastic scattering plays an important role in reducing the energy of fast neutrons in LMFBR's, and is therefore an important phenomenon to investigate. In inelastic scattering a neutron of energy $E$ enters the nucleus to form a compound nucleus. It is then re-emitted with a lower energy, leaving the original target nucleus in an excited state. The excited state subsequently decays 


\section{TABLE $\quad 3-7$}

THE CAPTURE RATES OF U-238 AND Na IN BLANKET NO. 2 USING VARIOUS DECAY GAMMA-RAYS FROM Np-239 AND Na-24

\begin{tabular}{|c|c|c|c|c|c|c|c|c|c|c|c|}
\hline \multicolumn{7}{|c|}{ RUN NUMBER } & \multicolumn{2}{|l|}{102} & \multicolumn{3}{|c|}{103} \\
\hline ISOTOPE & $\begin{array}{c}\mathrm{E \gamma} \\
(\mathrm{KeV})\end{array}$ & $\begin{array}{l}\mathrm{I} \gamma \\
(\%)\end{array}$ & $\begin{array}{c}S \gamma^{*} \\
\left(10^{-3}\right)\end{array}$ & $\begin{array}{l}\epsilon_{2} \Omega_{C^{*}} \\
\left(10^{-4}\right)\end{array}$ & Fy & $\mathrm{T}$ & $\mathrm{A} \gamma$ & $\mathrm{R}$ & $\mathrm{T}$ & A $\gamma$ & $R$ \\
\hline & 209.8 & 4.1 & 0.245 & 2.8 & 0.993 & 243.0 & 2621.3 & 0.386 & 363.8 & 4189.8 & 0.386 \\
\hline \multirow[t]{3}{*}{$\mathrm{Np}^{239}$} & 228.2 & 12.7 & 0.255 & 2.45 & 0.992 & $\approx 43.0$ & 9109.7 & 0.465 & 363.8 & 14680.5 & 0.507 \\
\hline & 277.6 & $14: 1$ & 0.271 & 1.90 & 0.992 & 243.0 & 13059.0 & 0.735 & 363.8 & 19007.5 & 0.727 \\
\hline & 334.3 & 2.0 & 0.290 & 1.65 & 0.992 & 243.0 & 1685.3 & 0.720 & 363.8 & 2393.5 & 0.708 \\
\hline $\mathrm{Na}^{24}$ & 1368.5 & 100.0 & 9.943 & 0.19 & 0.993 & 406.6 & 2167.5 & $0.288 \times 10^{-2}$ & 229.0 & 1466.8 & $0.346 \times 10^{-2}$ \\
\hline
\end{tabular}

* Calculated by Computer Codes, SELFN and SELFU. (Appendix E )

* Measured and calculated by INTEF. (Appendix D.). 
by gamma-ray emission. Inelastic scattering is impossible below the threshold energy or, rather, until the neutron energy exceeds $(A+1) / A$ times the energy of the first excited state, where $A$ is the mass number of the target nucleus.

The energy of the first and the second excited states of the materials of Blanket No, 2 are given in Table 3-8. In general the threshold energy for inelastic scattering decreases as $A$ increases. The excited levels of U-238 are noticeably lower than those of the other materials.

The inelastic scattering process can be analyzed by the measurement of the gamma-rays emitted by the excited target nucleus, since every inelastic scattering event is accompanied by the emission of gamma-rays, the energy of which corresponds to the excited levels of the target materials.

In a manner similar to that used for the calculation of capture reaction rates, the number of inelastic gammarays of interest having energy $E \gamma$ produced per second at the position $\underline{r}$ in the blanket is given by the following equation:

$$
\operatorname{C\gamma }(\underline{r})=\int_{E} N(\underline{r}) \sigma_{i}(E) \phi(E, \underline{r}) d E
$$


TABLE 3-8

ENERGY OF FIRST AND SECOND EXCITED STATES*

\begin{tabular}{|c|c|c|}
\hline NUCLEUS & $\begin{array}{l}\text { FIRST EXCITED } \\
\operatorname{STATE}(\mathrm{MeV})\end{array}$ & $\begin{array}{l}\text { SECOND EXCITED } \\
\text { STATE }(\mathrm{MeV})\end{array}$ \\
\hline $0-16$ & 6.05 & 6.13 \\
\hline $\mathrm{Na}^{23}$ & 0.438 & 2.08 \\
\hline $\mathrm{Cr}^{52}$ & 1.43 .4 & 2.37 \\
\hline $\mathrm{Fe}^{56}$ & 0.845 & 2.085 \\
\hline$u^{238}$ & 0.045 & 0.245 \\
\hline
\end{tabular}

* Nuclear Data Table 
As a first approximation we assume that the intensities of the $\gamma$-rays from the first excited level are proportional to the total inelastic scattering rate. Hence, the peak area of the gamma-rays having energy Er observed by the detector during the time interval, $\mathrm{T}$, is given by:

$$
A \gamma=R \cdot \varepsilon \gamma \cdot T
$$

where $\mathcal{E} \gamma=F \gamma \cdot S \gamma \cdot \epsilon \gamma \cdot \Omega$; the total efficiency- of the $\mathrm{Ge}(\mathrm{Li})$ detection system, which includes all correction factors and the intrinsic efficiency.

One can actually observe all the inelastic gammarays corresponding to the first excited states, in the gamma-ray spectra from Blanket No. 2, except for that of U-238. Since the energy levels of U-238 are very dense and are nearly describable as a continuum state even at the low energies involved, it is impossible to distinguish the peaks of the $\mathrm{U}-238$ inelastic gamma-rays against the very high background level in the low energy region of the gamma-ray spectra from Blanket No. 2 .

The gamma-rays from inelastic scattering have low energies except for those of $0^{16}$. This means that the measured total efficiency data can be a pplied only to $0^{16}$ 
inelastic scattering since the measured efficiency data for low energy gamma-rays are not available. The calculated total efficiency data must be used for the rest of the elements, where heterogeneous effects are again of concern.

3-5-2 Calculation of Total Efficiency for Low Energy Gamma-Rays

This section is mainly concerned with the calculation of the total efficiency for the low energy region of the gamma-rays from Blanket No. 2. The calculation can be done by using the intrinsic efficiency of the detector and the self-absorption correction factor of the system. Since the heterogeneous effect is large for low energy gamma-rays, the calculation of the total efficiency for the gamma-rays from $\mathrm{Na}_{2} \mathrm{CrO}_{4}$ should again be different from that for the U-fuel rod. First of all consider the capture rate of sodium in the blanket. As seen in the previous sections, the capture rate of sodium can be analyzed using both the prompt capture gamma-rays and the decay gamma-rays. This allows one to correct the intrinsic efficiency and the total efficiency.

Figure 3-8 shows the calculated total efficiency of the system for low energy gamma-rays. This efficiency data can be normalized to the measured total efficiency 


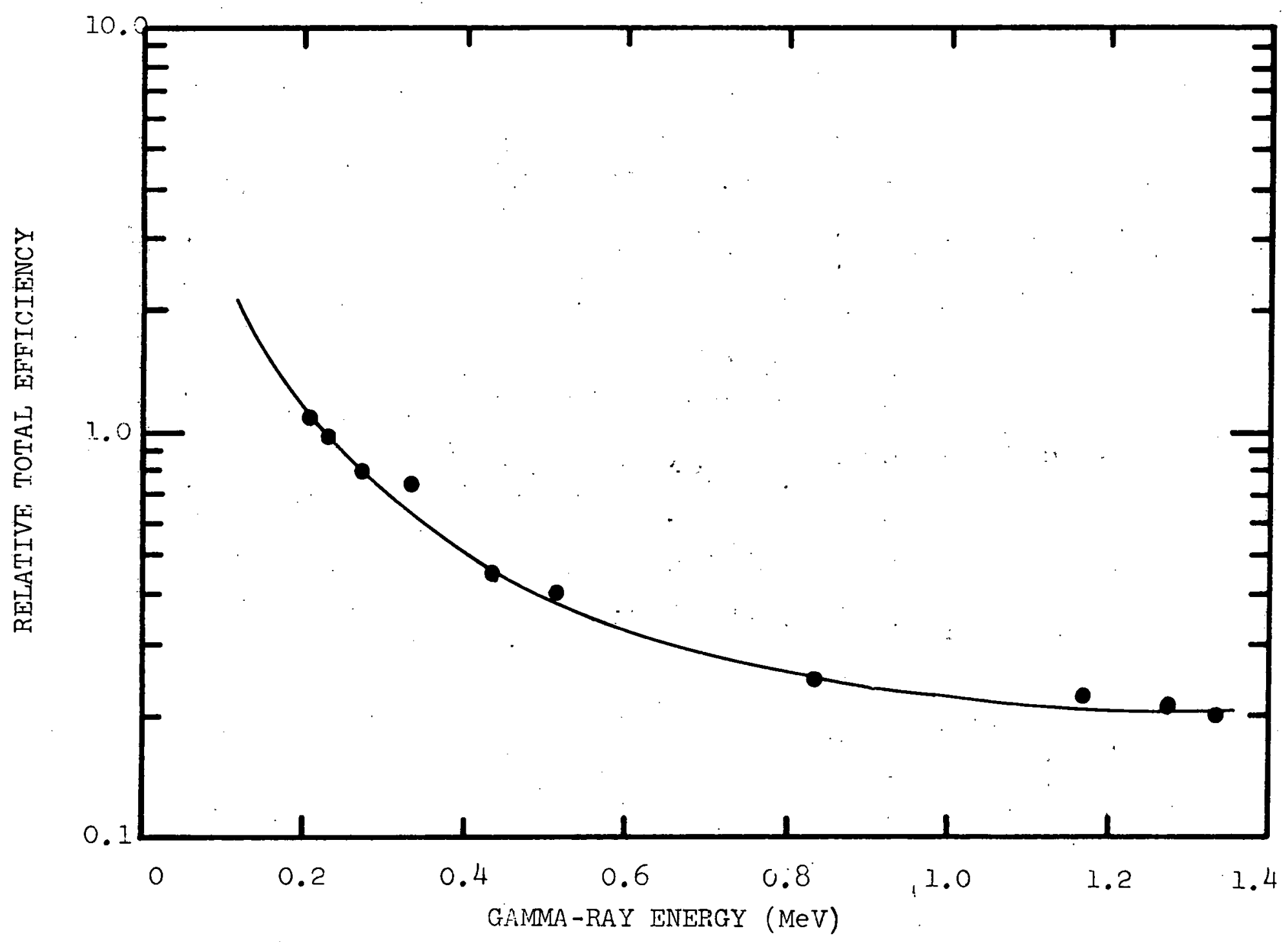

Fig. 3-8

Calculated Tota 1 Ffficiency for Low Energy Gamma-Rays, Which Originate from $\mathrm{Na}_{2} \mathrm{CrO}_{4}$ 
data obtained from iron lines. The normalization is done by manipulation of low and high energy capture gamma-ray lines from sodium and chromium. Table 3-9 shows the normalization factors which have been calculated using three sets of low-high energy prompt capture gamma-ray lines. Set No. 1 belongs to sodium and Sets No. 2 and No. 3 to chromium. The values for the total efficiency are read from Figs. 3-2 and 3-8. These normalization factors between the measured efficiency data for the high energy gamma-rays and the calculated efficiency data for the low energy gamma-rays made it possible to compare the reactions involving high energy gamma-rays with those of low energy gamma-rays.

The three sets of normalization factor calculations illustrate a very important point. The factors of Sets No. 1 and No. 3 are very similar to each other, and the factor of Set No. 2 is very different from the others. This shows that the yield intensity of some prompt capture gamma-rays changes with incident neutron energy, while the yield of others does not. This also confirms that in the calculation of the capture rates of sodium and chromium in section 3-3-2 the assumption of fixed yield for the specific gamma-rays in question was well-founded. 
TABLI $\quad 3-9$

CALCULATION OF NORMALIZATION FACTOR

\begin{tabular}{cccccc}
\hline SET & E $\gamma(\mathrm{KeV})$ & $\mathrm{I} \gamma(\%)$ & $\mathrm{A} \gamma$ & $\varepsilon \gamma$ & $\begin{array}{c}\text { Normalization } \\
\text { Factor }\end{array}$ \\
\hline \multirow{2}{*}{1} & 870.6 & 25.39 & 5838.0 & 2.3 & 4.14 \\
& 6395.4 & 25.69 & 1533.4 & 2.47 & 1 \\
\hline \multirow{2}{*}{2} & 835.1 & 24.04 & 6778.7 & 2.37 & 6.08 \\
\hline & 7939.3 & 11.41 & 446.2 & 2.00 & 1 \\
\hline & 835.1 & 24.04 & 6778.7 & 2.37 & 3.77 \\
\hline
\end{tabular}

* Average normalization factor is chosen to be 3.95 , which is the average value of Sets No. 1 and 3 . 
Table 3-10 gives the resultant inelastic scattering rates of $\mathrm{Na}, \mathrm{Cr}, \mathrm{Fe}$ and $\mathrm{O}$ in Blanket No. 2. Run No. 105 is analyzed for this purpose (Appendix I). 3-6 Fission in the Blanket 3-6-1 Short Lived Fission Product Gamma-Rays

The primary interest is focused on short-lived fission products in Blanket No. 2 for the purpose of investigating the fission rates in the blanket. Use of long-lived fission products raises a complicated : problem of unknown amount of accumulation of counts, and the use of exceptionally short-lived fission products, on the other hand, would raise the problem of poor counting statistics because of rapid decay.

Several leakage spectra were measured using the 2-in. diameter beam hole through the reflector of Blanket No. 2 (without any collimators) for about 24 hours of counting time. Runs No. 99, 102, and 103 in Appendix I are measured in this manner. For these spectra the blanket had been cooled for over a week prior to the 23 hours irradiation to get rid of the short-lived fission product gamma-rays due to previous irradiation, and it was then cooled for 100 minutes, counted for : 20 hours, and counted again during the next 43 hours. (Runs No. 102 and 103, respectively). The computer 
TABLE $3-10$

INELASTIC GAMMA-RAYS IN BLANKET NO. 2, RUN NO. 105 $\left(\mathrm{T}=8.24 \times 10^{4} \mathrm{sec}\right)$

\begin{tabular}{ccccc}
\hline Nuclide & E $\gamma(\mathrm{KeV})$ & $\mathrm{A} \gamma$ & $\sum_{\gamma}\left(10^{-4}\right)$ & $\mathrm{R}\left(10^{2}\right)$ \\
\hline $\mathrm{Na}^{23}$ & 438 & 3515.6 & 1.777 & 2.39 \\
$\mathrm{Cr}^{52}$ & 1433.9 & 2114.3 & 0.771 & 3.33 \\
$\mathrm{Fe}^{56}$ & 845 & 12530.3 & 0.932 & 16.32 \\
$\mathrm{O}^{16}$ & 6127.8 & 1072.4 & 0.250 & 5.19 \\
\hline
\end{tabular}


code GAMANL (R2) was used to analyze the data and the annihilation peak at $5 \mathrm{Il} \mathrm{KeV}$ and the $\mathrm{Ar}^{41}$ decay peak at $1.2936 \mathrm{KeV}$ are used for the purpose of energy. calibration. The identification of the peaks was based upon the data of Heath (Hl) and Gordon (G3). Table 3-Il gives the energies, the corresponding fission products and their half-lives for the principal lines of Run No. 99 (see Appendix I). More than 45 fission product gamma-ray lines were observed in the spectra of Runs No. 99, 102 and 103 from Blanket No. 2. Out of these many shortlived fission products only a few are really suitable for present purposes. Useful peaks are not chosen solely on the basis of the relative difference in their fission yield nor on the basis of the gamma-ray yield and the energy. Other parameters must be considered such as the fission yields of all the radioactive precursors that decay to the fission product of interest as well as the fission product itself, the decay constant, and also the yield of the gamma-ray. Also the half-lives of all precursors of the fission product of interest must be very short compared to that of the fission product itself.

Table 3-12 shows the decay chains which are selected for the present work. The numbers under the symbols 
TABLE $\cdot 3-11$.

SHORT-LIVED FISSION PRODUCT GAMMA-RAYS

FROM BLANKET NO. 2 .

\begin{tabular}{|c|c|c|c|c|c|}
\hline LINE & NUMBI & $E R^{*}$ & ENERGY $(\mathrm{KeV})$ & FISSION PRODUCT & HALF-LIFE \\
\hline & 1 & & 213.2 & $\mathrm{Ru}^{97}$ & $2.9 \mathrm{~d}$ \\
\hline & 2 & & 230.9 & $\mathrm{Te}^{132}$ & $78 \mathrm{~h}$ \\
\hline & 4 & & 251.5 & $\mathrm{Xe}^{135}$ & $9.2 \mathrm{~h}$ \\
\hline & 5 & & 264.9 & $Y^{93}$ & $10.1 \mathrm{~h}$ \\
\hline & 8 & . & 296.5 & $C e^{143}$ & $33 \mathrm{~h}$ \\
\hline & 9 & & $304: 3$ & $\mathrm{La}^{140}, \mathrm{Ba}^{140}$ & $40.2 h, 12.8 \mathrm{~d}$ \\
\hline & 16 & & 435.1 & $\mathrm{La}^{140}, \mathrm{Ba}^{140}$ & $40.2 \mathrm{~h}, 12.8 \mathrm{~d}$ \\
\hline & 18 & & 530.6 & $I^{133}$ & $20.8 \mathrm{~h}$ \\
\hline & 19 & & 541.6 & $\mathrm{Rh}^{101 \mathrm{~m}^{* *}}, \mathrm{Sb}^{129}$ & $4.5 \mathrm{~d}, 4.5 \mathrm{~h}$ \\
\hline & 23 & & 658.22 & $\mathrm{Nb}^{97}$ & $73 \mathrm{~m}$ \\
\hline & 24 & & 667.1 & $I^{132}, I^{126}, C_{S}{ }^{132}$ & $23 \mathrm{~h}, 13 \mathrm{~d}, 6.5 \mathrm{~d}$ \\
\hline & 26 & & 743.3 & $\mathrm{Nb}^{97 \mathrm{~m}}$ & $1.0 \mathrm{~min}$. \\
\hline
\end{tabular}

* The line numbers correspond to the peak numbers of Run No. 99 in Appendix I.

* The superscript $m$ refers to isometric states. 
TABLE 3-11

(concluded)

\begin{tabular}{cccc}
\hline LINE NUMBER & ENERGY(KeV) & FISSION PRODUCT & HALF-LIFE \\
\hline 28 & 772.2 & $\mathrm{I}^{132}$ & $2.3 \mathrm{~h}$ \\
29 & 811.8 & $\mathrm{Sb}^{129}$ & $4.5 \mathrm{~h}$ \\
30 & 816.5 & $\mathrm{La}^{140}, \mathrm{Ba}^{140}$ & $40.2 \mathrm{~h}, 12.8 \mathrm{~d}$ \\
33 & 846.8 & $\mathrm{I}^{134}$ & $52 \mathrm{~m}$ \\
37 & 1024.8 & $\mathrm{Sr}^{91}$ & $9.7 \mathrm{~h}$ \\
40 & 1147.6 & $\mathrm{Zr}^{97}, \mathrm{I}^{130}$ & $17 \mathrm{~h}, 12.4 \mathrm{~h}$ \\
42 & 1261.3 & $\mathrm{I}^{135}, \mathrm{Sb}^{129}$ & $6.7 \mathrm{~h}, 4.5 \mathrm{~h}$ \\
43 & 1282.5 & $\mathrm{I}^{135}$ & $6.7 \mathrm{~h}$ \\
47 & 1384.9 & $\mathrm{Sr}^{92}$ & $2.7 \mathrm{~h}$ \\
53 & 1587.6 & $\mathrm{Pr}^{142}$ & $19.2 \mathrm{~h}$ \\
54 & 1595.7 & $\mathrm{La}^{140}, \mathrm{Ba}^{140}$ & $40.2 \mathrm{~h}, 12.8 \mathrm{~d}$ \\
55 & 1677.5 & $\mathrm{I}^{135}$ & $6.7 \mathrm{~h}$ \\
\hline
\end{tabular}




\section{TABLE $3-12$}

FISSION PRODUCT DECAY CHAINS CF INTEREST TO THE PRESENT WORK

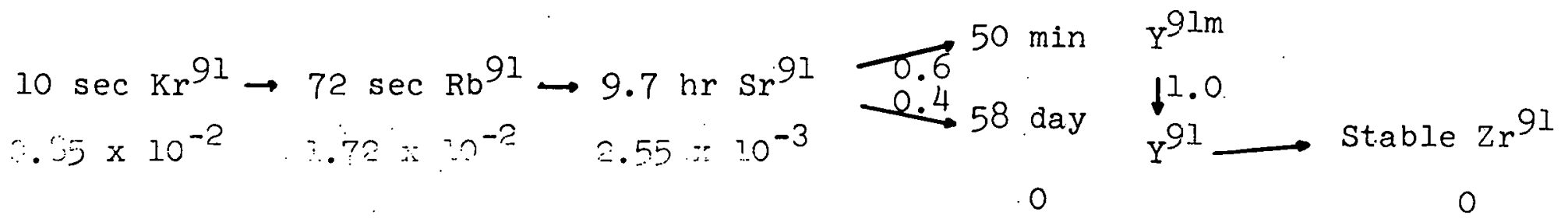

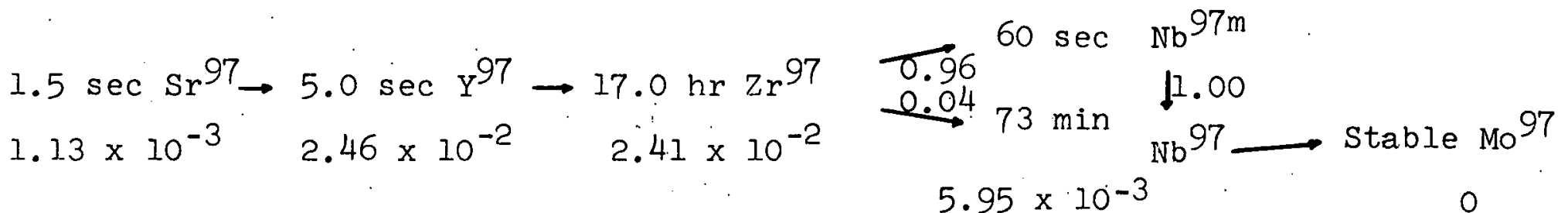

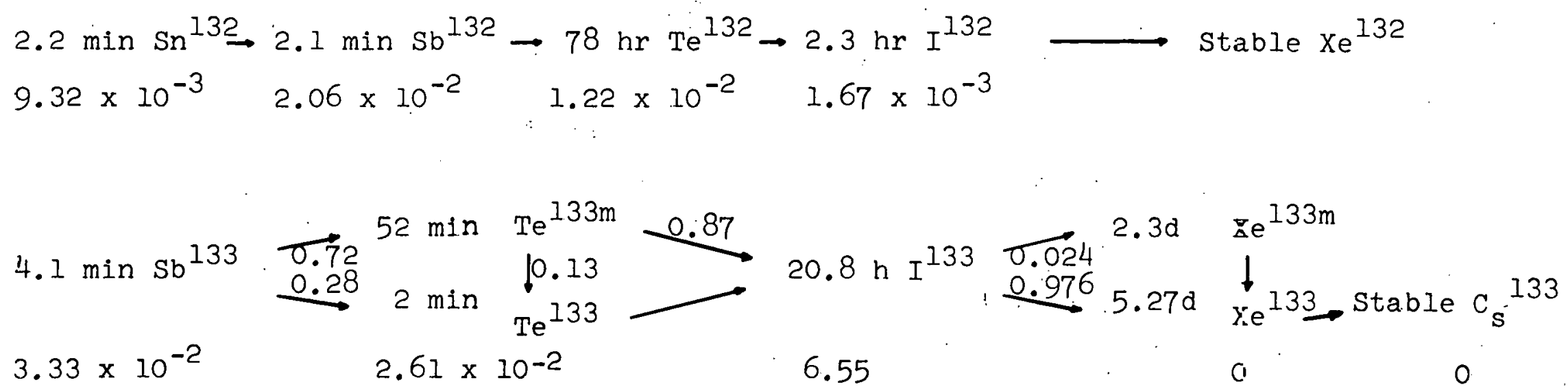


of the various isotopes are the yields of the fission products (TI). Table $3-13$ shows the principal lines of these fission products and their corresponding intensity yields. The peak areas of these lines of Run No. 99 are reported in the table.

3-6-2 Fission Rates

Short-lived fission product gamma-rays make possible the estimation of the total fission rate in Blanket No. 2 . There are two major contributions to the fission process in the blanket: from U-235 fission, and from U-238 fission. These two fissions will be treated as a total fission in the bianket in the present work.

As an example consider the fission product decay chain 97. The first two fission products, $\mathrm{sr}^{92}$ and $y^{97}$ have such short half-lives that their activity will die out within a few minutes. Then the $17 \mathrm{hr} . \mathrm{Zr}^{97}$ gives rise to $1147.6 \mathrm{KeV}$ gamma-rays when it decays into $\mathrm{Nb}^{97}$. Hence the total area recorded under this peak is:

$$
\mathrm{A} \gamma=\left(\frac{I \gamma}{I 00}\right) \cdot R \cdot S \gamma \cdot \mathrm{T} \cdot \mathrm{F} \gamma \cdot \mathbf{E} \gamma \cdot \Omega \cdot \mathrm{Y} \gamma .
$$

where

$R$ - is the total fission rate in the blanket, $\mathrm{Y} \gamma=\mathrm{Y}_{\mathrm{Sr}}+\mathrm{Y}_{\mathrm{Y}}+\mathrm{Y}_{\mathrm{Zr}}$; total fission yield up to $\mathrm{Zr}$, $T=\int_{t_{S}}^{t_{f}}\left(e^{-\lambda_{Z r} t}-e^{-\lambda Z_{r}\left(t_{I}+t\right)}\right) d t$, and the remaining terms are the same as defined before. 
TABLE $3-13$

PRINCIPAL FISSION PRODUCT GAMMAS OF INTEREST

\begin{tabular}{lccc}
\hline ISOTOPE & ENERGY $(\mathrm{KeV})$ & GAMMA - YIELD $(\%)$ & AREA $(\mathrm{A} \gamma)^{*}$ \\
\hline $\mathrm{Sr}^{91}$ & 1024.8 & 30 & $328.5 \pm 19 \%$ \\
$\mathrm{Zr}^{97}$ & 1146.7 & 2 & $144.2 \pm 28.1 \%$ \\
$\mathrm{Nb}^{97 \mathrm{~m}}$ & 743.3 & 100 & $1014.7 \pm 9.1 \%$ \\
$\mathrm{Nb}^{97}$ & 658.2 & 99 & $1861.6 \pm 7.1 \%$ \\
$\mathrm{Te}^{132}$ & 230.9 & 100 & $12485.1 \pm 2 \%$ \\
$\mathrm{I}^{132}$ & 667.1 & 95 & $797.8 \pm 7.1 \%$ \\
& 772.1 & 82 & $607.9 \pm 17.3 \%$ \\
$\mathrm{I}^{133}$ & 530.6 & 100 & $1112.5 \pm 9.8 \%$ \\
\hline Area data & are Run No. 99 (See Appendix I)
\end{tabular}


Therefore from the measured value of Ay for the 1147.6 $\mathrm{KeV}$ from $\mathrm{Zr}^{97}$, one can deduce the fission rate, $\mathrm{R}$. The gamma-ray of $\mathrm{Te}^{132}$ at $230.9 \mathrm{KeV}$ submerged by a strong peak of $\mathrm{Np}-239$ at $228.2 \mathrm{KeV}$, and $\mathrm{Sr}^{91}$ at 1024.8 $\mathrm{KeV}$ and $\mathrm{Zr}^{97}$ at $1146.7 \mathrm{KeV}$ introduce too $\mathrm{h} 1 \mathrm{gh}$ error ( $\pm 19 \%$ and $\pm 28.1 \%$ ). Therefore $\mathrm{Te}^{132} ; \mathrm{Sr}^{91}$ and $\mathrm{Zr}^{97}$ are not suitable for analysis.

Table 3-14 shows the gamma-rays chosen and the deduced total fission rates from different fission product gamma-rays. The estimated reaction rates are larger for $I^{132}$ than for the other peaks analyzed since the $I^{132}$ peak at $667.1 \mathrm{KeV}$ overlaps the $\mathrm{Cs}^{132}$ peak at the same energy.

Except for these two peaks, the calculated reaction rate varies from $0.7 \times 10^{9} / \mathrm{sec}$. to $1.6 \times 10^{9} / \mathrm{sec}$. The calculation was based upon thekey assumption that the yields of thermal U-235 fission apply in the present work. Most of the error introduced in this work is attributable to the difference of fission yields between thermal and fast neutron induced fission, between U-235 and $U-238$, and the uncertainty of the calculated selfabsorption correction factors.

\section{3-7 Summary}

So far neutron capture, inelastic scattering, and fission rates in Blanket No. 2 have been discussed in 
TABIE 3-14

FISSION RATE CALCULATIONS FOR RUN NO. 99

\begin{tabular}{lllllllll}
\hline ISOTOPE ENERGY $(\mathrm{KeV})$ & $\mathrm{Y} \gamma\left(10^{-2}\right) *$ & $\mathrm{I} \gamma(\%)$ & $\mathrm{T}$ & $\mathrm{S} \gamma\left(10^{-4}\right)$ & $\epsilon \gamma\left(10^{-4}\right)$ & \multicolumn{1}{c}{$\mathrm{A \gamma}$} & $\mathrm{R}\left(10^{9}\right)$ \\
\hline $\mathrm{Nb}^{97 \mathrm{~m}}$ & 743.3 & 5.49 & 100 & 407.42 & 3.01 & 2.14 & 1014.7 & 0.738 \\
$\mathrm{Nb}^{97}$ & 658.2 & 6.08 & 99 & 431.72 & 2.92 & 1.95 & 1861.6 & 1.255 \\
$\mathrm{I}^{132}$ & 667.1 & 4.38 & 95 & 167.27 & 2.93 & 1.97 & 797.8 & 1.98 \\
& 772.1 & 4.38 & 82 & 157.27 & 3.05 & 2.20 & 607.9 & 1.51 \\
$\mathrm{I}^{133}$ & 530.6 & 3.60 & 100 & 330.10 & 2.91 & 1.66 & 1112.5 & 1.68 \\
\hline
\end{tabular}

* The reference is for thermal neutron fission of U-235 (Reference G3) 
turn. The results are summarized in Table 3-15, and normalized and compared with theoretical and other experimental values (L5) in Table 3-16.

Even though the complete disappearance of the U-238 prompt capture lines made it difficult to deduce the capture rate of $\mathrm{U}-238$, it is encouraging that one can nevertheless deduce most of the pertinent neutron reaction rates in $\mathrm{Blanket}$ No. 2. The neutron capture rates of iron, chromium, sodium, and U-238 were analyzed in this chapter, and the inelastic scattering rates of sodium, chromium, iron and oxygen also were analyzed as well as the total fission rate in the blanket.

The measured reaction rate of iron is higher than calculated as might be expected, since iron is a major constituent in the reflector and shielding, and background interference is therefore possible in spite of the good collimation of the gamma-ray beam. The big difference in the capture rate of chromium is unexplainable. All that can be said is that for chromium the present method is preferable to foil activation, (T. Leung's method), since the $\mathrm{Cr}^{50}$ nuclide activated in the foil method is only $4.3 \%$ of natural chromium, while all chromium isotopes are represented in the prompt capture gamma spectrum. 
Leung assumed that the capture cross section in all chromium isotopes was the same in the energy range of interest, and thus his total chromium capture rate is just $1 / 0.043$ of the measured $\mathrm{Cr}^{50}$ capture rate. While this is undoubtedly more accurate than, say; scaling the activities proportional to the known thermal cross sections for the chromium isotopes, it is at best qualitative. The prompt capture gamma method on the other hand would be an accurate approach if capture gamma yields were either known at high energy or known not to change from their cataloged thermal values.

In regard to the high iron activation, it should be noted that the extraneous source must come from the near vicinity of the flight-path. This was demonstrated by carrying out a background run with the facility at power but with the 18 inch long steel plug inserted into the reflector beam hoie. The measured iron line background was found to be far less than the signal obtained during operation with the open flight-path. Thus the steel reflector or the iron laminae in port 12CHI are the likely sources of the background problem. The latter source could be eliminated by replacing the port shielding with a lead-lithiated plastic combination. 
TABLE $\quad 3-15$

REACTION RATES IN BLANKET NO. 2

\begin{tabular}{|c|c|c|}
\hline REACTION & RELATIVE REACTION RATI & REMARK (Gamma-rays) \\
\hline Fe $(n, \gamma)$ & $3.13-4.00$ & More than 20 prompt $\gamma$-peaks analyzed \\
\hline $\operatorname{Cr} \quad(n, \gamma)$ & $0.24-0.38$ & $835.1-, 7939.3-$ and $8884.1 \mathrm{KeV}$ \\
\hline $\mathrm{Na}(\mathrm{n}, \gamma)$. & $0.30-0.31$ & $870.6-, 6395.4-$ and $1368.7 \mathrm{KeV}$ \\
\hline $\mathrm{U}^{238}(\mathrm{n}, \gamma)$ & $50.7-72.7$ & $\begin{array}{l}\mathrm{Np}-239 \text { decay } \gamma-\text { rays at } 209.8- \\
228.2-, 277.6-, \text { and } 334.3-\mathrm{KeV}\end{array}$ \\
\hline $\mathrm{Na}\left(n, n^{\prime}\right)$ & $2.39 \pm 16.7 \%$ & $438.0 \mathrm{KeV}$ \\
\hline $\mathrm{Cr} \quad\left(n, n^{\prime}\right)$ & $3.33 \pm 14.0 \%$ & $1433.9 \mathrm{KeV}$ \\
\hline Fe $\left(n, n^{\prime}\right)$ & $16.32 \pm 4.5 \%$ & $845.0 \mathrm{KeV}$ \\
\hline $0 \quad\left(n, n^{\prime}\right)$ & $5.19 \pm 16.7 \%$ & $6127.8 \mathrm{KeV}$ \\
\hline$U \quad(n, f)$ & $7.38-19.8$ & $\begin{array}{l}\text { F1ssion Product Decay Gamma-Rays } \\
\text { from } \mathrm{Nb}^{97 \mathrm{~m}}, \mathrm{Nb}^{97}, \mathrm{I}^{132} \text { and } \mathrm{I}^{133}\end{array}$ \\
\hline
\end{tabular}


TABLE $3-16$

NEUTRON BALANCE: IN BLANKET NO. 2

\begin{tabular}{|c|c|c|c|c|c|c|c|}
\hline REACTION & ANISN & & & LEUNG ( $\mathrm{LS}^{\mathrm{S}}$ & & & PRESENT WORK: \\
\hline $\mathrm{U}^{238}(\mathrm{n}, \gamma)^{*}$ & 1.0000 & & & 1.0000 & & & 1.0000 \\
\hline Fe $(n, \gamma)$ & 0.0036 & & & -- & & & $0.0346 \pm 0.0042$ \\
\hline Na $(n, \gamma)$ & 0.0025 & & & 0.0022 & & & $0.0029 \pm 0.0001$ \\
\hline $\operatorname{Cr}(n, \gamma)$ & 0.0650 & & $\therefore$ & 0.0740 & & & $0.0030 \pm 0.0007$ \\
\hline$U^{235}(n, \gamma)$ & 0.0016 & & & -- & & & --- \\
\hline$U^{238}(n, f)$ & $0.0485 i$ & & & $0.0520_{3}$ & & & \\
\hline$U^{235}(n, f)$ & $0.0584^{1}$ & .1069 & & $0.0610^{]}$ & 0.1 .130 & & $0.1326 \pm 0.0612$ \\
\hline $\mathrm{Na}\left(\mathrm{n}, \mathrm{n}^{\prime}\right)$ & 0.0271 & & & - & & & $0.0232 \pm 0.0039$ \\
\hline $\operatorname{Cr}(n, n !)$ & 0.0081 & & & -- & & & $0.0324 \pm 0.0046$ \\
\hline $\mathrm{Fe} \quad\left(n, n^{\prime}\right)$ & 0.0418 & & & -- & & $\therefore$ & $0.1590 \pm 0.0071$ \\
\hline $0 \quad\left(n, n^{\prime}\right)$ & 0.0001 & : & & -- & & & $0.0504 \pm 0.0084$ \\
\hline
\end{tabular}

* All values reported relative to $U-2 \Xi 8$. 
The capture reaction rates of sodium and the fission rate are in good agreement with calculated values and with T. Leung's results from the foil activation method. (Note that the results in Table 3-16 are normalized to selfshielded in-rod U-238 activities; thus Leung's entries have been re-normalized to account for the fact that the values tabulated in Ref. (L5) are for unshielded U-238 foils)

The inelastic scattering rates are in poor agreement with the theoretical results except for sodium. This is mainly because the inelastic scattering cross-section is not the same as the production cross-section for the gammaray corresponding to the first excited level. other sources of error are the uncertainties in the high energy neutron spectrum deep in the blanket and in the inelastic scattering cross-sections. 


\section{Chapter IV \\ DETERMINATION OF HYDROGEN CONTENT OF \\ LMFBR BLANKET MATERIALS}

\section{4-1 Introduction}

Since the hydrogen content of the LMFBR blanket materials has a significant effect upon the neutron energy spectrum in the blanket assembly (FI), it is very important to know exactly how much hydrogen is contained in 1ts component materials. Because of the high neutron slowing down power of hydrogen the moisture which is absorbed or contained in the materials causes depletion of fast neutrons and enhancement of epithermal neutrons in the blanket. I. Forbes (II) has calculated the effect of hydrogen in the blanket and converter assembly.

The ANISN code was used to make 16-group, 1-D, $\mathrm{S}_{8}$ calculations of the neutron capture and fission rates of U-238 through the blanket and steel reflector of Blanket No. 2. The U-238 capture (a typical capture reaction) and fission (a typical threshold reaction) rates were calculated for chromate water contents of $0.0 \mathrm{w} / 0,0.1 \mathrm{w} / 0,0.2 \mathrm{w} / 0$ and $0.5 \mathrm{w} / 0$. Figure $4-1$ shows, for a given sodium chromate water content, the 


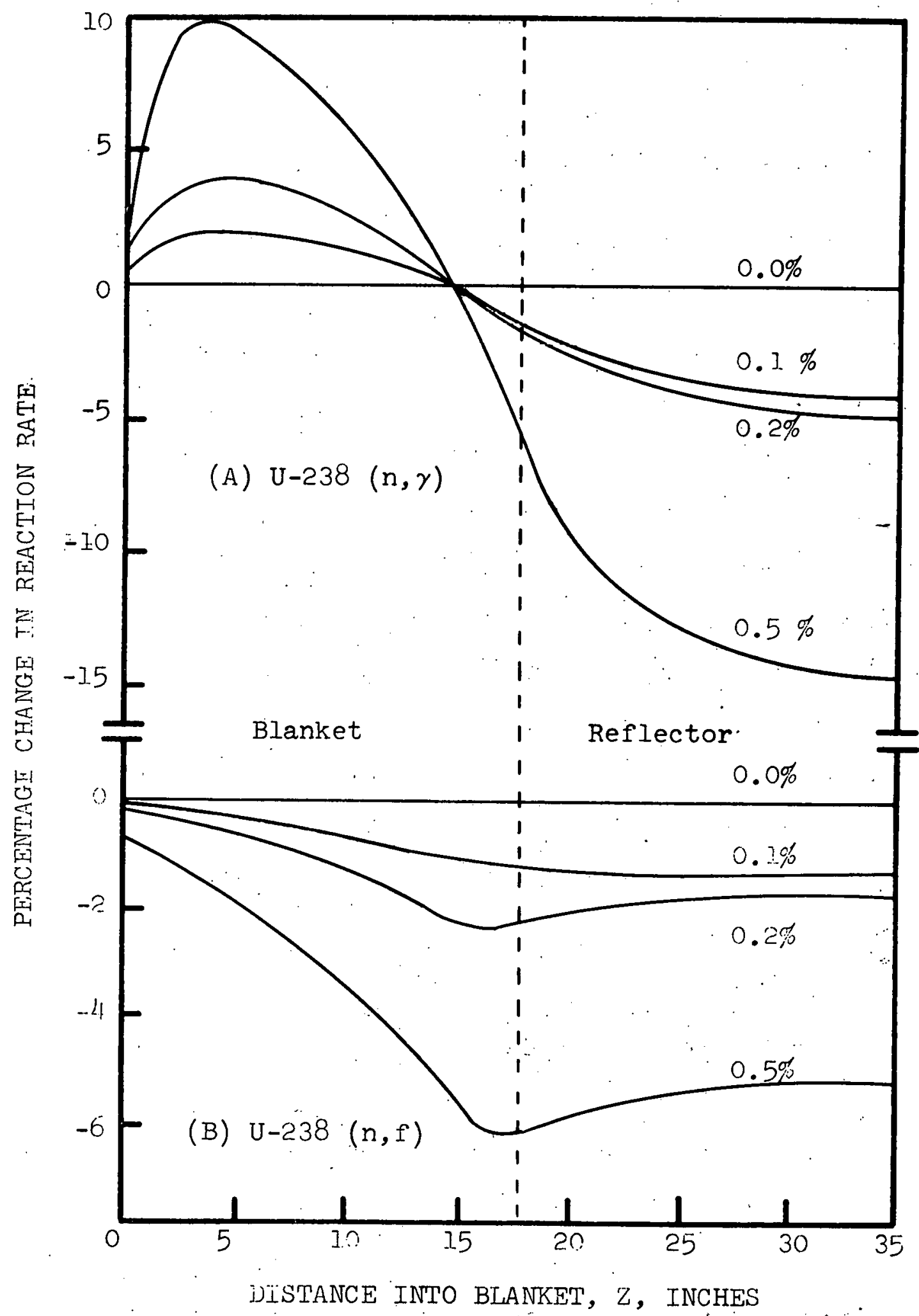

Fig. 4-1 Percentage Error in Reaction Rates in Blanket No. 2 as a Function of the Sodium Chromate Water Content Weight Percentage 
amount by which the U-238 capture and fission rates through Blanket No. 2 differ from the zero water content case. It is seen that the error in the capture and fission rates due to the hydrogen content of the chromate is reduced to about the limit of precision in the experimental determination of the activities only when the water content of the sodium chromate is $0.1 \mathrm{w} / \mathrm{o}$ or less. As can be seen, a good knowledge of the moisture content of blanket materials is quite important since as little as $0.1 \mathrm{w} / 0 \mathrm{H}_{2} \mathrm{O}$ can change the $\mathrm{U}-238$ capture rate by as much as $\pm 5 \%$.

The good energy resolution of $\mathrm{Ge}(\mathrm{L} i)$ detectors makes possible the accurate measurement of moisture content using the prompt capture gamma-ray of hydrogen. In fact, in most thermal neutron prompt capture analysis carried out at MIT the hydrogen characteristic line was always so prominent that it was used as an energy calibration line in almost every experiment.

This chapter investigates the possible application of prompt activation analysis in competition with physical/chemical analyses, to assess the accuracy and efficiency of the method, not only for the present specific application, but also for more general industrial or scientific applications. The prompt 
activation analysis method has been improved both as regards the detector itself, and the associated detection system.

\section{4-2 Theory}

When a proton captures a neutron, the binding energy of the deuteron appears as a gamma-ray of about $2.223 \mathrm{MeV}$. The probability of this process, i.e., the thermal neutron capture cross-section of hydrogen is (H6):

$$
\sigma_{c}(2200)=(0.332 \pm 0.002) b
$$

However the capture cross-section of hydrogen decreases so rapidly with energy that high energy neutrons cannot be effectively used to analyze the hydrogen content in a material.

The only possible prompt gamma-radiation emitted as a result of thermal neutron capture in the hydrogen is at $2.223 \mathrm{MeV}$, since there is no other excited bound state in the deuteron. The precise energy of this hydrogen gamma-ray is very important to the present work in many respects. Table 4-1 shows the energy of the thermal neutron prompt capture gamma-rays of hydrogen reported by various experimenters. The value of 2.223 $\mathrm{MeV}$ is obviously a well-established and reliable result. As already noted, the characteristic hydrogen peak 
TABIE 4-1

THE ENERGY OF THE HYDROGEN THERMAL NEUTRON CAPTURE GAMMA-RA V LINE

\begin{tabular}{lll}
\hline \multicolumn{1}{c}{ Reference } & \multicolumn{1}{c}{ Instrument } & Energy (KeV) \\
\hline Bell and Elliot (B2, B3) & $\begin{array}{l}\text { Magnetic B Spectrometer with a } \\
\text { Uranium Photoelectric Converter }\end{array}$ & 2229 \\
Monahan et al. (MI) & NaI Scintillation Spectrometer & $2217.2 \pm 1.5$ \\
Motz et al. (M2) & Magnetic Compton Spectrometer & $2223.0 \pm 1.0$ \\
Chupp et al. (CI) & 2m Bent Crystal Spectrometer & $2223.3 \pm 1.5$ \\
Kazi et al. (KI) & 2m Bent Crystal Spectrometer & $2224.2 \pm 1.5$ \\
Knowles (K2) & Double Flat Crystal Spectrometer & $2223.18 \pm 0.14$ \\
Greenwood et al. (G6) & Ge(Li) Detector & $2223.29 \pm 0.07$ \\
\hline
\end{tabular}


at $2.223 \mathrm{MeV}$ is a sensitive indicator of hydrogen content. The total area under this peak is:

$$
A \gamma=\int_{V} d v \int_{T} d t \int_{E} d E \Gamma(E) \Sigma_{C}(\underline{r}, E) \phi(\underline{r}, \dot{E}, t) \cdot \varepsilon_{\gamma}(4-I)
$$

where

$\mathrm{V}$ - is the volume of the sample,

$\mathrm{T}$ - is the neutron irradiation time,

$F(E)$ - is the number of characteristic prompt gamma-rays emitted per capture of a neutron having energy $E$,

$\Sigma_{c}(\underline{r}, E)$ - is the macroscopic neutron capture crosssection of hydrogen at the position $\underline{r}$ and at neutron energy $E$, $\phi(\underline{r}, E, t)-$ is the neutron flux in which the sample is immersed at the position $\underline{r}$ for neutrons having energy $E$, at time $t$, and

$\varepsilon_{\gamma}$ - is the total gamma-ray detection efficiency of the experimental system, which includes all correction factors, the solid angle factor, and the intrinsic detection efficiency.

For the present application we may confidently assume that the irradiated sample is small enough to: neglect the variation of reaction rate with position, and the neutron flux is also nearly constant throughout 
the time interval $T$. Equation 4-3 may then be written:

$$
A \gamma=\mathrm{VT} \varepsilon_{r} \int_{E} \Gamma(E) \Sigma_{c}(E) \varnothing(E) d E
$$

by making use of the definitioris:

$$
\int_{T} d t \phi(\underline{r}, E, t) \equiv \phi(\underline{r}, E): T
$$

and

$$
\int_{V} d V \Sigma_{c}(\underline{r}, E) \phi(\underline{r}, E) \equiv \Sigma_{c}(E) \cdot \phi(E) \cdot V
$$

Since a highly thermal neutron flux is used, we may also assume the energy spectrum is Maxweliian, and thereby define an effective averaged one-group cross-section. Hence, the intensity of the hydrogen characteristic prompt capture gamma-ray line at $2.223 \mathrm{MeV}$ is given by the following equation:

$$
A \gamma=V \cdot T \cdot \varepsilon \gamma \cdot \Gamma^{t h} \cdot \Sigma_{c}^{t h} \cdot \phi^{t h},
$$

where the superscript "th" refers to the thermal neutrons. The macroscopic capture cross-section of hydrogen is given by:

$$
\Sigma_{c}^{t h}=2 \cdot N_{A} \cdot \frac{m}{M \cdot V} \sigma_{c}^{t h}
$$


where

$$
\begin{aligned}
& \mathrm{N}_{A} \text { - is Avogadro's number, } \\
& \mathrm{m} \quad \text { - is the total equivalent } \mathrm{H}_{2} \mathrm{O} \text { mass in the } \\
& \text { sample, } \\
& \mathrm{M} \text { - is the molecular weight of water, and } \\
& \sigma_{C}{ }^{\text {th }} \text { - is the microscopic thermal neutron capture } \\
& \text { cross-section. }
\end{aligned}
$$

From Eq. 4-5 the area, A $\gamma$, may be written:

$$
\mathrm{A} \gamma=\mathrm{C} \gamma \cdot \mathrm{m}
$$

where

$$
C \gamma=2 \cdot \mathrm{N}_{\mathrm{A}} \cdot \mathrm{T} \cdot \boldsymbol{\varepsilon} \gamma \cdot \boldsymbol{I}^{\mathrm{th}} \cdot \phi^{\mathrm{th}} \cdot \frac{\mathrm{I}}{\mathrm{M}} \cdot \sigma_{\mathrm{C}}{ }^{\mathrm{th}} .
$$

Now we can see from Eq. 4-7 that the intensity of the hydrogen characteristic. line is directly proportional to the moisture content of a material. Since $\mathrm{C} \gamma$ contains a variety of factors subject to uncertainty, it is preferable to employ a comparison technique to obtain absolute results. The same sample is reirradiated with a small known amount of hydrogeneous material, $\Delta \mathrm{m}$, added to it, for the same interval, T. The new peak area of the hydrogen characteristic line becomes:

$$
A \gamma^{\prime}=(m+\Delta m) C \gamma
$$


Hence, from these two consecutive experiments hydrogen content in a material is given by:

$$
m=\frac{\Delta m}{R-1},
$$

where

$\Delta \mathrm{m}$ - is the mass of the known amount of hydrogeneous material added, and

$R$ - is the ratio of the two photopeak areas, $A^{\prime} / A$. 4-3 Experimental Setup and Procedure 4-3-1 Irradiation Fac1lity and Detection Setup The prompt activation analysis facilities using thermal neutron beam port 4THI of the MIT Research Reactor have been described in detail by $Y$. Hukai (H3), T. Harper ( $\mathrm{H} 4$ ) and V. Orphan (OI). The front irradiation facility was used in this experiment. This facility was constructed by T. Harper (H4) as part of his doctoral thesis research. The primary shielding material used is heavy concrete, together with masonite and boral which are used to thermalize and capture stray neutrons. Lead is used for shielding and collimation of gammarays in the access ports of the facility. The thermal neutron beam coming from the reactor port can be interrupted just preceding the irradiation position by a boral and lead shutter. 
The neutrons from the reactor core are scattered by a graphite plug, 3-7/8 in. diameter and 40 in. long, placed inside the $4 \mathrm{TH} l$ beam port, which is tangential to the reactor core tank; the resulting scattered neutrons form a beam for the external irradiation facilities. The cadmium ratio for gold at the front irradiation position was measured and found to be 54 (H3). To reduce the gamma-ray background from the beam port, a $1-1 / 2$ in. long lead plug was placed in the beam port.

The sample is inserted into the irradiation position through one of the side access ports in the shielding; the prompt capture gamma-rays coming from the sample. are collimated vertically by a set of lead collimators. Figure 4-2 shows a schematic view of the sample pusition which can be adjusted horizontally.

A planar, hexagonal-shaped, 7C.C.Ge(Li) detector with 5 C.C. of active volume was used (Ref. H3). The electronics associated with the 7C.C. detector was the standard arrangement for free mode operation. The signal from the $\mathrm{Ge}(\mathrm{Li})$ cryostat is amplified by a CI-1408C preamplifier which is connected through the pre-amplifier head, followed by a CI-1417 main amplifier, and thereafter fed into a Nuclear Data.ND-16IF 4096 Channel Analyzer. $-500 \overline{\mathrm{V}}$ was supplied as the detector 


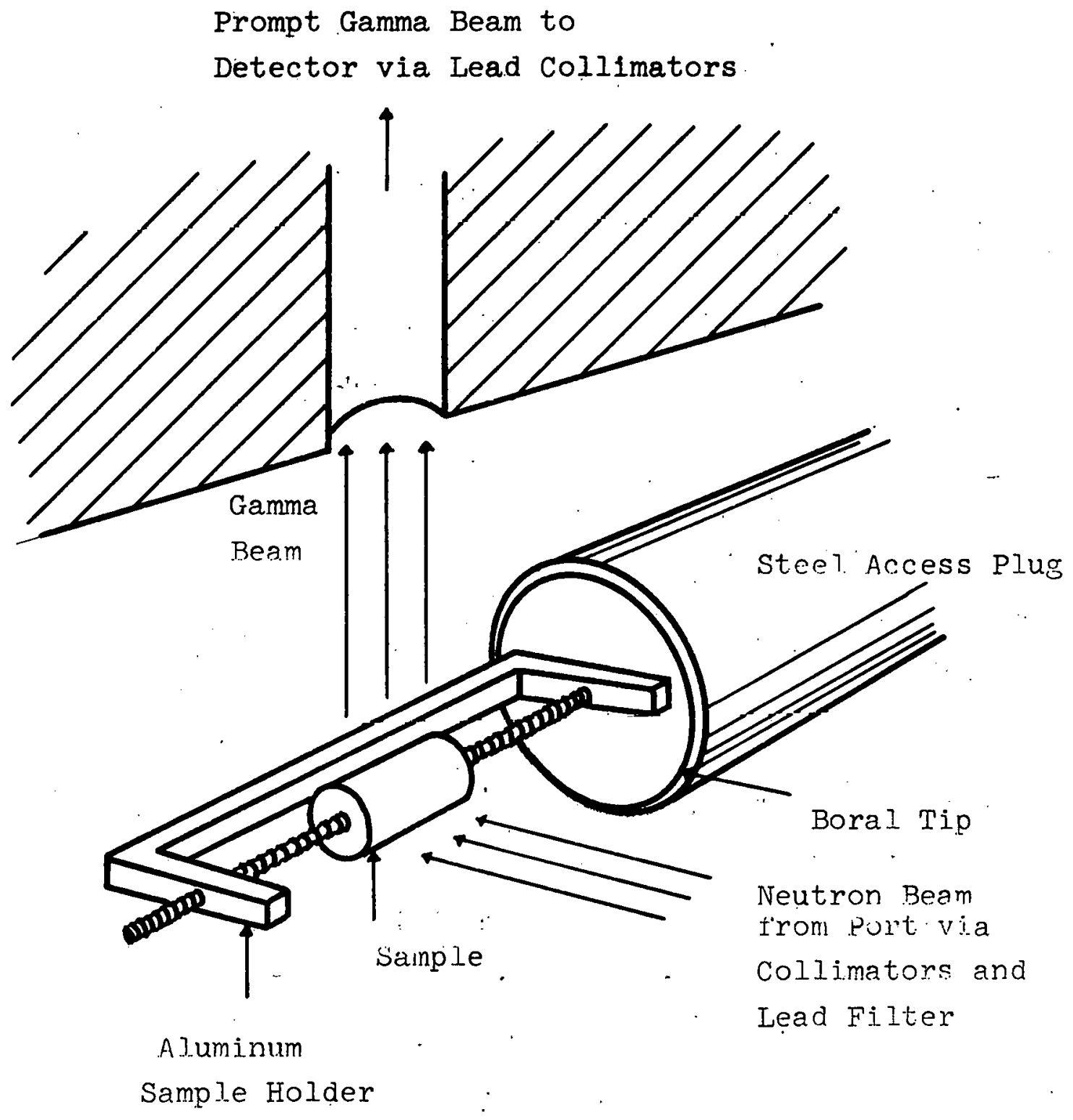

Fig. 4-2 Schematic of Sample Position in

Front Irradiation Facility 
bias voltage.

4-3-2 Operating Procedure

Crucible drying experiments showed that the water content of the technical grade "anhydrous" sodịum chromate, as delivered by the supplier, had a water content of about $0.8 \mathrm{w} / \mathrm{o}$. This was clearly unacceptable in terms of its effect upon blanket neutronics, and was sufficient motivation for confirmation of this analysis by an independent method.

The sodium chromate samples were prepared in $3 / 4$ in. diameter and 2 in. long aluminum cans, and sealed airtight. The uniformity of the neutron flux over the region occupied by the sample position in the front facility was checked by Y. Hukai (H3). The size of the present sample was kept within the allowable limits assuring uniformity of the neutron flux. To add a.. known amount of hydrogen to the sample, Mylar tape was used. During each irradiation the fluctuation of the incident neutron flux and the variation of the irradiation time interval was monitored using another characteristic Iine from the sample. Right adjacent to the hydrogen characteristic line at $2.223 \mathrm{MeV}$, chromium has a prompt capture line at $2.239 \mathrm{MeV}(\mathrm{R} 3)$. The total thermal crosssection of chromium is $3.10 \mathrm{~b}$ and the capture gamma-ray 
yield per 100 thermal neutron captures is 7.45 at this energy. This chromium prompt capture line at 2.239 MeV was used as a monitor line to check the fluctuation of the neutron flux and the irradiation time interval between two successive experiments. Taking the ratio of the intensity of the chromium lines between two experiments will cancel out all such fluctuation between two experiments. A background run was also performed: no significant peak was found at $2.223 \mathrm{MeV}$, which is encouraging in terms of use of the prompt activation method to measure the moisture content in a material using this irradiation facility. The monitoring method of this experiment using another adjacent line such as chromium at $2.239 \mathrm{MeV}$ is more reliable and more convenient than the foil monitoring method employed in most óther studies using this facility. Since the monitor line originates from the sample itself, no error can be introduced by position difference, which is not always true in the foil monitoring method. Further the foil method requires extra work, such as keeping track of irradiation, cooling and counting times, weighing the foils, etc.. 4-4 Data Analysis Figure 4-3 shows the prompt capture gamma-ray 
104

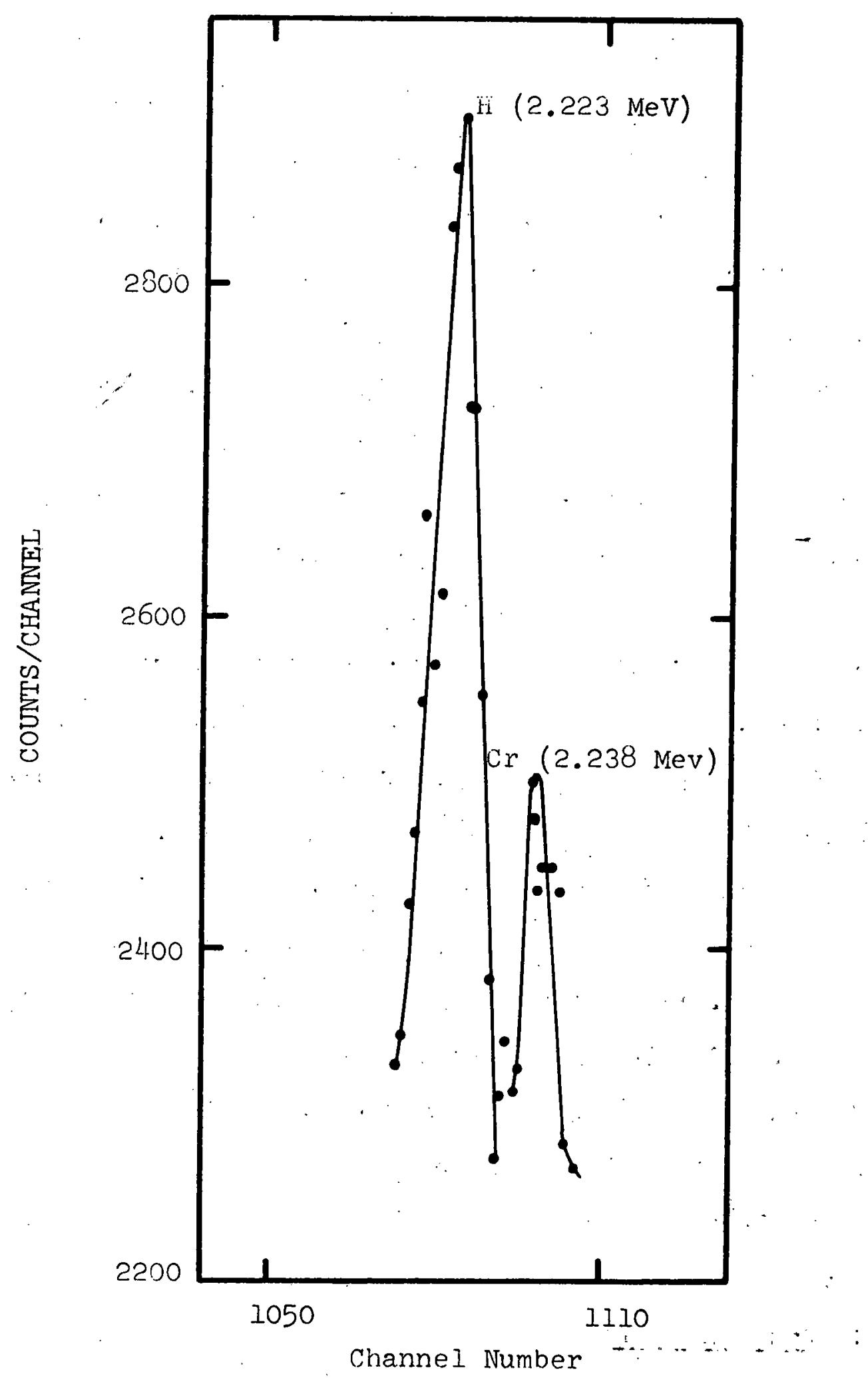

'Fig. 4-3 Spectrum of $\mathrm{H}$ and $\mathrm{Cr}$ of $\mathrm{Na}_{2} \mathrm{CrO}_{4}$ with

Known Amount of Hydrogen Added,

Run No. 38 


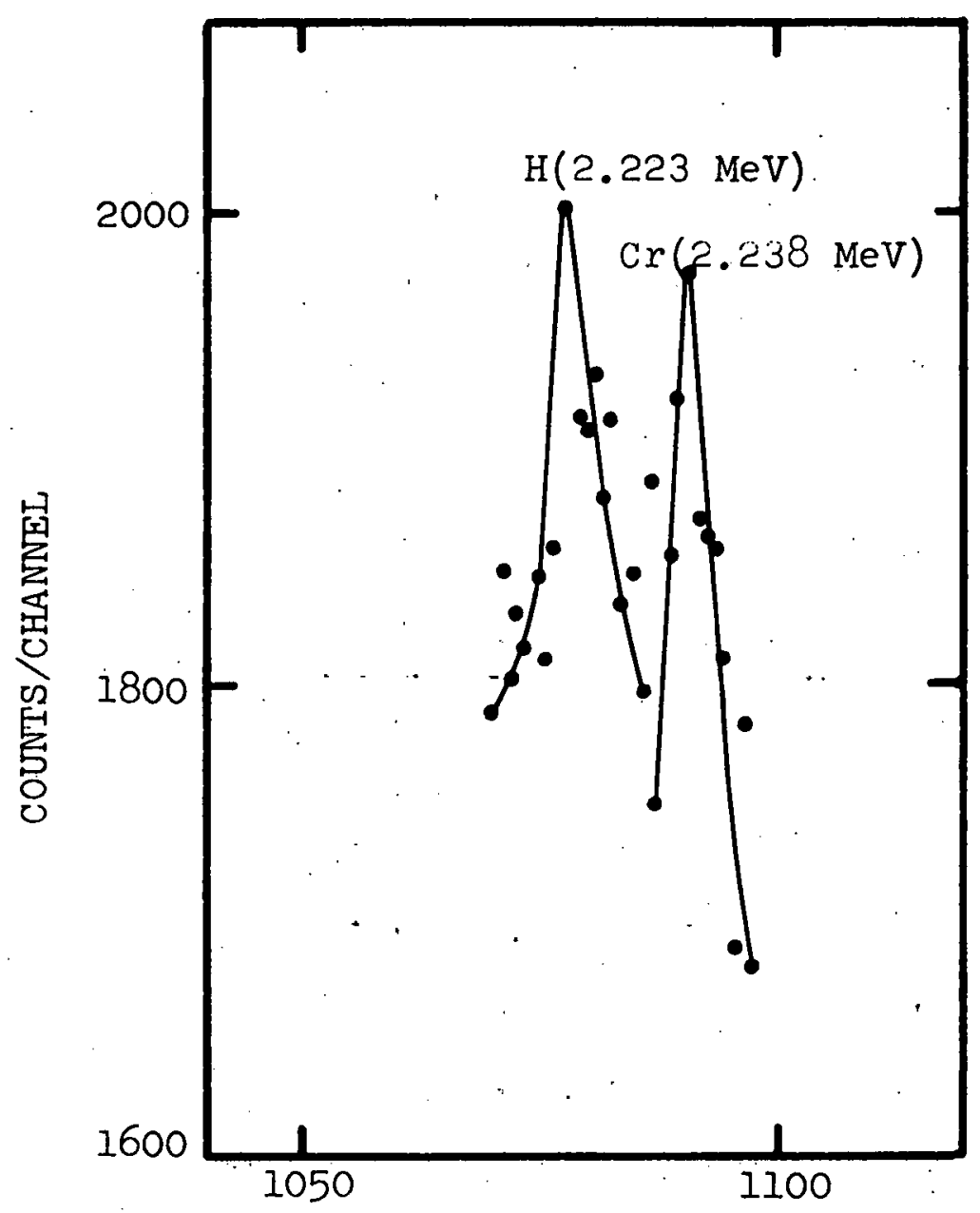

CHANNEL NUMBER

Fig. 4-4 Spectrum of $\mathrm{H}$ and $\mathrm{Cr}$ of $\mathrm{Na}_{2} \mathrm{CrO}_{4}$, Run No. 40 
spectrum of $\mathrm{Na}_{2} \mathrm{C}_{2} \mathrm{O}_{4}$ with Mylar tape added. The high peak on the left is the H-line at $2.223 \mathrm{MeV}$, and the small peak at the right is the $\mathrm{Cr}$-line at $2.239 \mathrm{MeV}$. Figure 4-4 shows the same lines from $\mathrm{Na}_{2} \mathrm{C}_{\mathrm{r}} \mathrm{O}_{4}$ without any Mylar tape. "Both'experiments had the same irradiation time of 468 minutes. Because of the addition of a small amount of hydrogeneous material, we can see the large augmentation of the $\mathrm{H}$-line at $2.223 \mathrm{MeV}$. The $\mathrm{Na}_{2} \mathrm{C}_{r} \mathrm{O}_{4}$ sample consisted of:

\begin{tabular}{llr}
$\mathrm{Na}_{2} \mathrm{C}_{\mathrm{r}} \mathrm{O}_{4}$ & & $12.3718 \mathrm{gm}$ \\
$\mathrm{Al}-\mathrm{can}$ & $\ddots$ & $11.6660 \mathrm{gm}$ \\
Mylar Tape & & $0.7710 \mathrm{gm}$ \\
\hline Total & $\ddots$ & $24.8088 \mathrm{gm}$
\end{tabular}

The Mylar tape was added at the center of the aluminum can. The first run (Run No. 38) was made with the prepared sample with Mylar tape added, and the following run (Run No. 40) was made with the sample after taking off the Mylar tape.' A background run (Run No. 39) was also made with an aluminum can without any $\mathrm{Na}_{2} \mathrm{C}_{\mathrm{C}_{4}} \mathrm{O}_{4}$ sample. The background subtracted total counts of the $\mathrm{H}-\mathrm{Iine}$ and $\mathrm{Cr}-\mathrm{Iine}$ are as follows: 


\begin{tabular}{lcc}
\hline Run Number & 38 & 40 \\
\hline Description & with Mylar & without Mylar \\
$C_{r}$-Monitor line & $1042 \pm 32$ & $1133 \pm 34$ \\
H-IIne & $3889 \pm 62$ & $443 \pm 21$ \\
\hline
\end{tabular}

Hence the amount of hydrogen which is contained in $12.3718 \mathrm{gm}$ of $\mathrm{Na}_{2} \mathrm{C}_{\mathrm{r}_{4}}$ sample was $0.095 \pm 0.015 \mathrm{gm}$ (Mylar Tape equivalent; the hydrogen content in Mylar tape is almost the same as that in the same weight of waterr). As a result the moisture content in the $\mathrm{Na}_{2} \mathrm{CrO}_{4}$ was $0.77 \pm 0.12 \mathrm{w} / \mathrm{O}$ (Mylar-tape equivalent). The water content of the sodium chromate delivered by the supplier was determined to be $0.83 \pm 0.04 \mathrm{w} / \mathrm{o}$ from the crucible drying tests. In order to reduce this contamination the entire shipment of sodium chromate was baked at $400^{\circ} \mathrm{F}$ for eight hours in a domestic electric oven. Since sodium chromate has a low dehydration temperature (about $300^{\circ} \mathrm{F}$ ), a simple oven drying procedure was good enough. After baking, the weight of the sodium chromate was measured and found to be decreased by about $0.75 \%$. The dried chromate was ground and stored in sealed cans prior to loading it into the subassemblies of Blanket No. 2. Samples from the dried sodium chromate loaded into the blanket subassemblies 
were confirmed to be $\leq 0.1 \mathrm{w} / \mathrm{o}$ by both crucible drying tests and prompt activation methods. No resolvable line was observed using the prompt activation method and dried sodium chromate samples. It is estimated that as little as $0.1 \mathrm{w} / \mathrm{o}$ would have been detectable.

This prompt activation analysis of hydrogen insures the detection of all possible hydrogen in $\mathrm{Na}_{2} \mathrm{C}_{r} \mathrm{O}_{4}$, while the crucible drying tests could miss bound hydrogen, such as in $\mathrm{NaOH}$ impurity, a very likely contaminert in sodium chromate. Furthermore it was also very encouraging that no $\mathrm{H}$-Iine was detectable in the gamma spectra from Blanket No. 2. 


\section{Chapter V \\ DETERMINATION OF THE NEUTRON LEAKAGE SPECTRUM \\ FROM BLANKET NO. 2}

\section{5-1 Introduction}

The following two chapters will focus on the analysis of information that can be obtained from the spectra from $\mathrm{Ge}(\mathrm{Li})$ detectors by means other than convectional photo-peak analysis, namely measurement of neutron energy spectra and the continuous gamma-ray spectra of the Compton recoil continum. The measurement of the neutron energy spectrum leaking from Blanket No. 2 will be discussed in this chapter.

Reactor physics has as its chief concern the process of neutron multiplication, absorption and leakage. Consequently the spatial and energy variation of the neutron population is of considerable interest. These dual objectives are in fact the subject of two. other detailed investigations on Blanket No. 2 (L5,03). In effect the present work parallels these other approaches in all respects,but with therestriction that a gamma-ray spectrometer is the tool rather than foil activation, He-3 or Proton recoil methods. Reaction rate measurements were already discussed in a previous chapter; the measurement of the meutron spectrum is 
the concern of the present one.

The evaluation of techniques for the measurement of the leakage neutron spectrum from Blanket No.2 has focused on three methods,

1. An energy shift-method using low $-Z$ materials,

2. A prompt activation method, and

3. Tt.e analysis of $\mathrm{Ge}$ interval conversion spectra at $691.4 \mathrm{KeV}$.

The first two methods will be discussed in the last section of this chapter and the third method wili be developed and applied. The final technique developed will permit measurement of the neutron energy spectrum above $700 \mathrm{KeV}$ using a comparison method in which spectra are unfolded by comparison with the neution spectrum froma $\mathrm{C} f-252$ standard neutron source. All response kernel matrices (which will be discussed in Section 5-2) will be compared to and corrected by the spectra from $\mathrm{Cf}-252$. To effect the unfolding a series of linear equations will be solved by the so-called iterative unfolding method (G7). 5-2 Theory

5-2-1 Internal Conversion Spectrum at $691.4 \mathrm{KeV}$

In most experiments using Ge( $(i i)$ gamma-ray detectors for fast neutron prompt capture analysis two spectral 
lines undergo broadening in a manner which is not. observed in applications involving thermal neutron activation analysis. These two spectral lines are at $595 \mathrm{KeV}$ and $691.4 \mathrm{KeV}$, and are well-broadened on the high energy side compared with any other photo-peaks. In particular the peak at $691.4 \mathrm{KeV}$ shows broadening of almost 100 $\mathrm{KeV}$ (FWHM is less than $4 \mathrm{KeV}$ ), so much that it will be referred to as a "spectrum" rather as a "peak" in this chapter.

The spectrum at $691.4 \mathrm{KeV}$ is produced by the internal conversion of $\mathrm{Ge}^{72}$ nuclei excited by inelastic scattering events with the neutrons incident on the Ge(Li) detector. Internal conversion is an alternative mode of deexcitation which always competes with gamma-ray. emission if the nuclear excitation energy is small and the angular momentum change is large (E4). In the case of the $\mathrm{Ge}^{72}$ nucleus since $\mathrm{O}^{+} \rightarrow \mathrm{O}^{+}$transitions are forbidden in any radiative process, all the transition must proceed by internal conversion (E5). The other spectrum, at $595 \mathrm{KeV}$ is, on the other hand, produced by gamma-ray. emission of $\mathrm{Ge}^{74}$, which is caused by inelastic scattering reactions with the fast neutrons incident on the Ge( $L i)$ detector.

This de-excitation energy from $\mathrm{Ge}^{72}$ or $\mathrm{Ge}^{74}$ is 
accompanied by the ionization energy deposited by recoil Ge atoms, which broadens the peaks at $595 \mathrm{KeV}$ and 691.4 $\mathrm{KeV}$. The appreciable broadening of both the gamma-ray lines from $\mathrm{Ge}^{74}$ at $595 \mathrm{KeV}$ and the conversion electron Iine from $\mathrm{Ge}^{72}$ at $691.4 \mathrm{KeV}$ is a well substantiated phenomenon reported previously by several investigators, including Hukai at MIT (C2, C3, H3). In this chapter the focus will be mainly on the analysis of the internal conversion spectra of $\mathrm{Ge}^{72}$ at $691.4 \mathrm{KeV}$, since the $\mathrm{Ge}-74$ line is contaminated by superposition of photons from higher energy events.

\section{5-2:2 Broadening of $691.4 \mathrm{KeV}$ Spectrum}

When a $\mathrm{Ge}(\mathrm{Li})$ detector is exposed to fast neutrons, inelastic scattering events will occur and the $\mathrm{Ge}^{72}$ nuclei will recoil to conserve momentum. The observed line-broadening is due to the amount of electronic excitation produced by the recoiling Ge atom when the de-excitation of the excited state is coincident in time (within the charge collection time of the detecting system, typically about $20 \mu \mathrm{sec}$ ) with the slowing down of the recoiling Ge atom (which occurs in $0.3 \mu \mathrm{sec}$ ). The lower limit to the observable energy loss is fixed by the intrinsic energy resolution of $\mathrm{Ge}(\mathrm{Li})$ detectors and 
their associated electronics. Actually the spectra at $691.4 \mathrm{keV}$ will be given by summation of the hole-electron pairs produced in the Ge crystal by the $691.4 \mathrm{keV}$ internal conversion transition and the hole-electron pairs produced by the recoiling Ge atom.

Jones et al. studied the energy lost to ionization by recoiling Ge atoms in Ge(Li) detectors (C2, C3). They supported Lindhand's conjecture (L3) of an electronic stopping power proportional to $\mathrm{v}$ for particle energies down to very low energy. There was a satisfactory agreement with the theory of Lindhard et al.

Figure 5-1 shows the comparison of the experimental values of Jones et al. with the theoretical curves which represent the solution of the equations given by Lindhanrd et al.

Therefore the electronic energy loss of the recoiling atoms can be expressed by

$$
E^{\prime \prime}=f\left(E^{\prime}\right)
$$

where $E^{\prime \prime}$ is the energy lost ionization and $E^{\prime}$ is the energy of the recoiling atom. In other words, the broadened energy, $\mathrm{E}^{\prime \prime}$, of $\mathrm{Ge}^{72}$ internal conversion spectra is a known function of the energy of the recoiling atom, $E^{\prime}$. In the present work the experimental formula of 


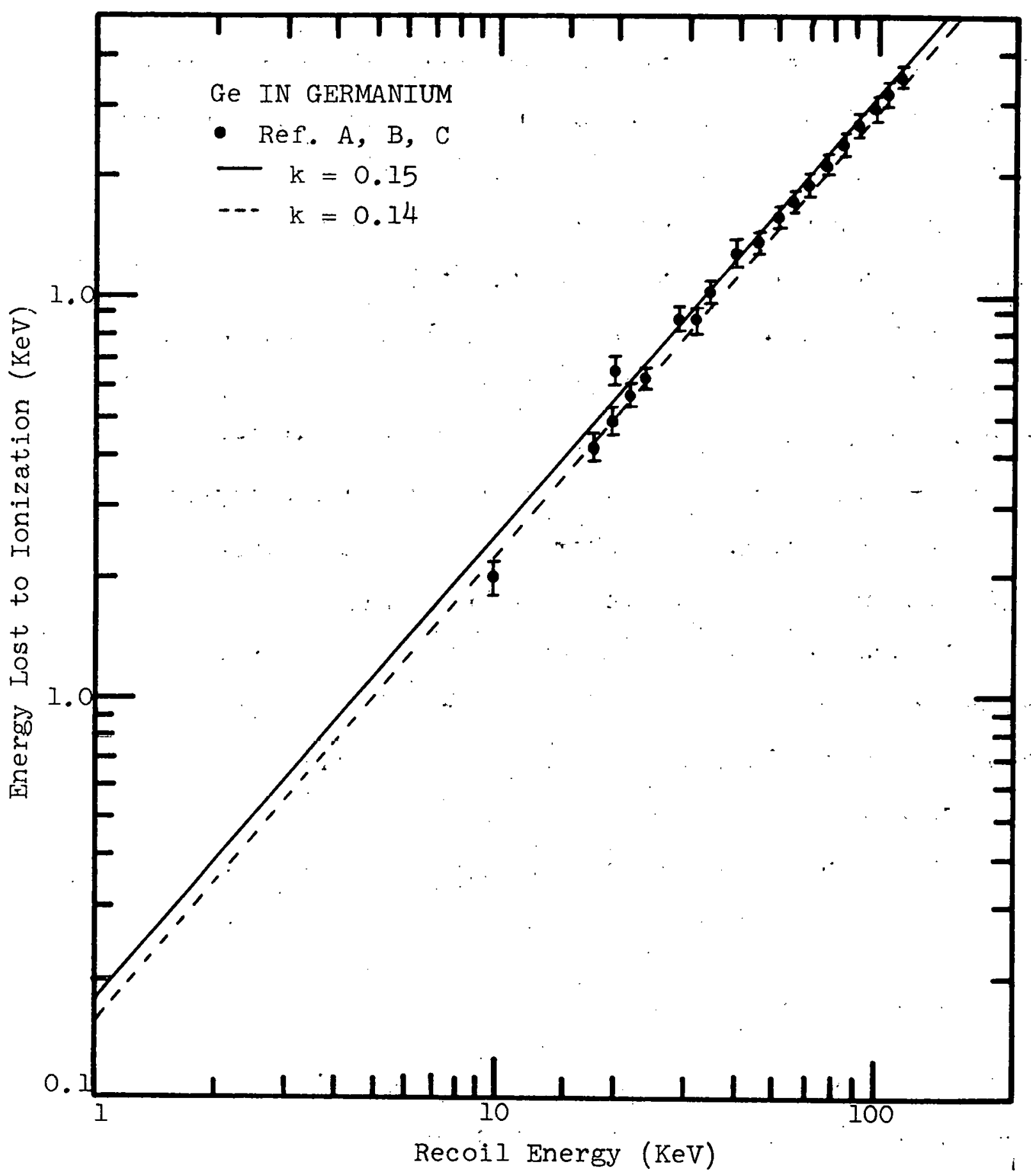

Fig. 5-1 The Energy Lost to Ionization vs. the Recoil Energy of: Germanium.

Ref. A - Chaseman et al., Phys. Rev. Letters, 20; 1430 (1968) Ref. B - Chaseman et al., Phys. Rev. Letters, 15, 245 (1965) Ref. C - Chaseman et al., Phys. Rev. 154, 239 (1967) 
Jones et al. is used for this functional relationship. This semi-empirical formula is:

$$
E^{\prime \prime}=a\left(E^{\prime}\right)^{b}
$$

where

$$
a=0.170 \text { and } b=1.163
$$

5-2-3 Integral Equation Formulation

Let $\phi(E, \Omega$ be the steady-state angular neutron flux at energy $E$ in the direction $\Omega$. Here $\phi$ possesses the customary units of an angular flux, namely neutrons per unit area per unit time per unit energy per steradian. It is also assumed that the angular flux does not vary rapidly over the region of the Ge(Li) detector. For example, when the detector is far away from the neutron source we can assume the flux is independent of the angular direction. Let $d N\left(\Omega^{\prime}\right)$ be the number of Ge recoils per unit time arising in the solid angle I at $\Omega$. Introducing the differential cross-section per steradian per free neutron, $a_{j} / \alpha \Omega$, one has

$$
d N\left(\Omega^{\prime}\right)=N d \Omega \int_{E} \frac{d \sigma}{d \Omega}\left(E, \Omega^{i}\right) \phi(E) d E
$$

where $\mathrm{N}$ is the total number of $\mathrm{Ge}^{72}$ nuclei in the detector, and all solid angles are in the center of 
mass system. Since in a beam experiment the neutron flux is mono-directional, $\Omega$ simply denotes the cosine of the angle between the original neutron direction and the germanium nucleus recoil direction. Consequently one can transform from the variable $\Omega$ to the final state germanium nucleus recoil energy E', since the observable production rate of germanium nuclei obtained from a $\mathrm{Ge}(\mathrm{Li})$ detector is the number of germanium nuclei per unit time arriving in a final state energy interval $d E^{\prime}$, at $E^{\prime}$. In this case, Eq. 5-3 becomes

$$
d N\left(E^{\prime}\right)=N d E^{\prime} \int P\left(E \rightarrow E^{\prime}\right) \not(E) d E,
$$

where

$$
P\left(E \rightarrow E^{\prime}\right)=\frac{d \sigma}{d \Omega}\left(E, \Omega^{\prime}\right) \frac{\partial \Omega}{\partial E^{\prime}}
$$

is defined so that $\mathrm{P}\left(\mathrm{E} \rightarrow \mathrm{E}^{\prime}\right) d \mathrm{E}^{\prime}$ is the probability that a germanium nucleus will be recoiled with an energy between $E^{\prime}$ and $E^{\prime}+d E^{\prime}$ when the nucleus is struck by. a neutron of energy $E$.

This representation of the recoiling kernal can be written in the following form:

$$
P\left(E \rightarrow E^{\prime}\right)=-\frac{2 \pi \sigma(E, \theta)}{\sigma(E)} \cdot \frac{d \mu}{d E^{\prime}}
$$


where

$$
\begin{aligned}
d \Omega & =-2 \pi d \mu \\
\sigma(E) & =\int_{4 \pi} \sigma(E,(\text { (A) }) d \Omega \text { and }
\end{aligned}
$$

$\sigma(E,(1))$ is the differential recoiling cross-section of the germanium nucleus by neutrons having energy $E$ through an angle (A) .

Hence the spectra of the recoil germanium atom becomes

$$
I_{R}\left(E^{\prime}\right)=\frac{d N\left(E^{\prime}\right)}{d E^{\prime}}
$$

or

$$
I_{R}\left(E^{\prime}\right)=N \int P\left(E, E^{\prime}\right) \varnothing(E) \cdot d E
$$

Equation $5-13$ is the desired integral equation relating the germanium recoil spectrum, $I_{R}\left(E^{\prime}\right)$, to the neutron energy spectrum, $\phi(E)$, and Eq. 5-2 relates the measured ionization loss by the stopping of recoiling germanium atoms in the germanium, E", with the energy of the recoiled germanium, $E^{\prime}$.

In the next section the recoiling kernel, $P\left(E \rightarrow E^{\prime}\right)$, will be discussed. The integral equation is solved numerically using a computer code, SPECT (see Appendix F). 


\section{5-2:4 Recoiling Kernel $P\left(E \rightarrow E^{\prime}\right)$}

Since the $\mathrm{Ge}^{72}$ nucleus has well resolved and widely spaced inelastic levels at $691.4-, 835$ - kev, etc.*, classical collision theory can be applied as discussed by Donohew (DI). While this may not be adequate at high energies, this kernel will be corrected by using a measured response function.

By applying the conservation of mass-energy and of linear momentum to the collision between a neutron of unit mass and a nucleus of mass $A$, a relationship between the neutron energy before and after the collision and the recoiling angle can be derived. The velocity of the recoiling nucleus after the collision is given by:

$$
\left.\left(V^{\prime}\right)^{2}=V_{d}^{2}+V_{c m}^{2}-2 V_{d} V_{c m} \cos (5),-1 I\right)
$$

where

$\mathrm{V}^{\prime}$ - is the velocity of the recoiling nucleus in the laboratory system,

$\mathrm{V}_{\mathrm{d}}$ - is the velocity of the recoiling nucleus in the center-of-mass system,

$\mathrm{V}_{\mathrm{cm}}$ - is the velocity of the center of mass in the laboratory system, and

(1) - is the scattering angle in the center of mass * Nuclear Data Tables system. 
Using the conservation of mass-energy, the following relationship can be obtained:

$$
\frac{E^{\prime}}{E}=\frac{A}{(A+I)^{2}}\left[D^{2}+1-2 D \mu\right]
$$

where

$$
\begin{aligned}
& \mu=\cos \text {; (A) - scattering angle in the C-system, } \\
& D=\sqrt{I+\frac{A+I}{A} \frac{Q}{E}}
\end{aligned}
$$

$E$ - is the energy of neutrons before the collision in the L-system,

$E^{\prime}$ - is the energy of recoiling nuclei after the collizion in the L-system, and

Q - is the energy of the inelastic level.

Hence, the differentiation of $E^{\prime}$ with respect to $\mu$ becomes:

$$
\frac{d E^{\prime}}{d \mu}=-\frac{2 D A}{(A+1)^{2}} \cdot E
$$

And the possible energy range should lie between the values of (A) $=0$ and $(1)=2 \pi$. In other words the energy of the recoiling nuclei is confined to be:

$$
\alpha_{1} E \leq E^{\prime} \leq \alpha_{2} E
$$

where 


$$
\begin{aligned}
& \alpha_{1}=A\left(\frac{D-I}{A+I}\right)^{2} \text { and } \\
& \alpha_{2}=A\left(\frac{D+I}{A+I}\right)^{2} .
\end{aligned}
$$

Hence the recoiling kernel, Equation 5-6, becomes:

$$
\mathrm{P}\left(\mathrm{E} \rightarrow \mathrm{E}^{\prime}\right)=\left[\begin{array}{cl}
\frac{\pi(\mathrm{A}+1)^{2} \sigma(E, \Theta)}{\mathrm{D} \cdot \mathrm{A} \cdot \mathrm{E} \cap(\mathrm{E})} & \mathrm{a}_{1} E \leq E^{\prime} \leq \alpha_{2} E \\
0 & \text { otherwise }(5-18)
\end{array}\right.
$$

For isotropic recoil within the C-system, the recoiling kernel becomes:

$$
P\left(E \quad E^{\prime}\right)=\left[\begin{array}{lr}
\frac{(A+1)^{2}}{4 A D E} & \alpha_{1} E \leq E^{\prime} \leq \alpha_{2} E \\
0 & \text { otherwise } \quad(5-19)
\end{array}\right.
$$

This assumption is very good near the threshold energy. The distribution becomes increasingly anisotropic as the energy of the incident neutrons increases. However this is not a bad initial assumption. This anisotropic effect will also be corrected for by introducing the response function using a $\mathrm{Cf}-252$ standard neutron source. 5-2-5 Response Diagonal Matrix and Constraints

In the numerical treatment of integral equations of the type encompassing Eq. 5-10, it is customary to introduce a matrix representation. For a particular 
value $E^{\prime}=E_{i}^{\prime}, E q .5-10$ can be written as follows:

$$
I_{R}\left(E_{i}^{\prime}\right)=N \int_{E^{\prime} / \alpha_{2}}^{E^{\prime} / \alpha_{1}} P\left(E, E_{i}^{\prime}\right) \varnothing(E) d E
$$

where all the terms are the same as those defined before. As a first approximation we can assume the neutron flux $\varnothing(E)$ is a known function within the energy interval. This function may be any appropriate trial function (such as a constant, a linear function, a cubic polynomial, etc). For this work a constant average flux is assumed in the energy interval. Then Eq. 5-20 can be approximated as:

$$
I_{R}\left(E_{i}^{\prime}\right)=\sum_{j=1}^{N} C_{i j} \phi_{j}
$$

where

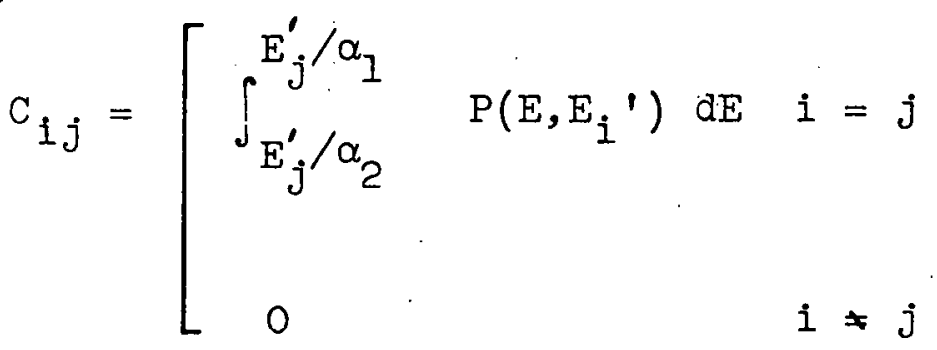

Using constraints imposed by collision theory, one can obtain a particularly advantageous representation. More specifically, given a recoil germanium atom of energy $E^{\prime}$, the permissible range of initial neutron energies is bound from below by $(E 6)$; 


$$
(E)_{\min }=\frac{I}{4 E^{\top} \cdot A}:\left\{Q+E^{\prime} \cdot(I+A)\right\}^{2},
$$

where

$$
E^{\prime} \geq \frac{Q}{I+A} \quad ; \quad \text { the threshold energy. }
$$

This constraint helps solve the matrix equation by trial

and error. The bound energy in E-space, which corresponds to $E_{j}^{\prime}$ in the $E^{\prime}$-space, is

$$
\left(E_{j}\right)_{\min }=\frac{I}{4 E_{j}^{\prime} \cdot A} \quad\left\{Q+E_{j}^{\prime}(I+A)\right\} .
$$

Finally, the matrix equation may be expressed:

$$
\left[I_{R}\right]=\cdots[C][\varnothing] \text {, }
$$

where

$$
\begin{aligned}
& I_{R}=\operatorname{col}\left\{I_{R I}, I_{R 2}, \ldots \ldots \ldots I_{R N}\right\}, \\
& C=\operatorname{dia}\left\{C_{1 I}, C_{22}, \ldots \ldots . C_{N N}\right\}, \text { and } \\
& \varnothing=\operatorname{col}\left\{\phi_{1}, \phi_{2}, \ldots \ldots \ldots \phi_{N}\right\} .
\end{aligned}
$$

When we assume that the neutron energy spectra from $\mathrm{Cf}-252, \phi_{\mathrm{S}}(E)$, is known, the matrix equation becomes

$$
\left[I_{R}\right]=[C][\varnothing] \quad S \text {. }
$$

where $[\varnothing]_{S}=$ the known flux column vector from $\mathrm{Cf}-252$. The measured spectra can be written:

$$
\left[I_{R}\right]=[C]_{M}[\varnothing]_{M}
$$

where $[\phi]_{M}=$ the neutron flux column vector deduced from the measured $\left[I_{R}\right]$ spectrum vector using $\mathrm{Cf}-252$ 
irradiation. From Eqs. 5-26 and 5-27, the corrected response diagonal matrix becomes

$$
[C]=[C]_{M}[\lambda]^{T}
$$

where the elements of $[\lambda]$ are

$$
\lambda_{i i}=\frac{\phi_{M i}}{\phi_{S i}}
$$

This corrected response diagonal matrix will enable us to cover the entire energy range of the incident neutrons without incurring distortion due to analytical or numerical shortcomings of the unfolding technique. The actual solution is accomplished by a computer code SPECT, described in Appendix F.

\section{5-3 Data Analys is}

\section{5-3-1 Cf-252 Standard Neutron Source}

It is very. important to have an exact neutron spectrum available as a reference. In common with a growing number of other applications $\mathrm{C} f-252$ has been selected for the present work. In $\mathrm{C} f-252$ spontaneous fission, an average of approximately two neutrons per fragment are released. These neutrons have the typical shape of fission neutron spectra. For fission sources, the spectrum shape is frequently taken to be Maxwellian, 


$$
\not(E) \sim \sqrt{E} \exp \left(-\frac{E}{T_{C}}\right)
$$

so that it is completely specified by the single parameter $\mathrm{T}_{\mathrm{c}}$, the Maxwellian temperature. A number of measurements of this parameter have been made for $\mathrm{Cf}-252$ fission neutron sources, and these are summarized in Table 5-1. The fourth temperature listed in Table 5-1 is an average of three earlier measurements. The last three are.the results of more recent studies, obtained using techniques very similar to one another. It can be seen that there is wide disagreement between No. 6 and No. 5 or No. 7. For the present work the temperature from. H. Condé or L. Green $\left(T_{c}=1.390 \pm 0.04\right)$ is chosen because these results represent more recent work. The Cf-252 neutron source has a most probable energy of $700 \mathrm{KeV}$ and a $2.58 \mathrm{yr}$. effective half-life, and the average number of neutrons emitted per fission is 3.77 . The use of a $\mathrm{Cf}-252$ source to deduce the response function of the detecting system and to correct the calculated response matrix is described in the following section. 5-3-? Experimental Procedure and Data Analysis The runs with $\mathrm{Cf}-252$ neutron sources were made in the well-isolated, low background accelerator vault in 


\section{TABL: 5-1}

Cf-252 Fission Neutron Spectrum Parameter

\begin{tabular}{ccc}
\hline Number & $\mathrm{T}_{\mathrm{c}}$ (Maxwellian Temp., MeV) & Reference \\
\hline 1 & $1.57 \pm 0.05$ & $\mathrm{S3}:$ \\
2 & $1.37 \pm 0.03$ & $\mathrm{~B} 4$ \\
3 & $1.42 \pm 0.05$ & $\mathrm{~S} 3$ \\
4 & $1.466 \pm 0.100$ & $\mathrm{R} 4$ \\
5 & $1.390 \pm 0.04$ & $\mathrm{C} 4$ \\
6 & 1.592 & $\mathrm{M} 3$ \\
7 & $1.390 \pm 0.04$ & $\mathrm{G} 8$ \\
& & \\
\hline
\end{tabular}


building NW-12 at MIT. The source was placed in the middle of the $31.5 \mathrm{ft}$. by $19 \mathrm{ft}$. by $12 \mathrm{ft}$. high vault in order to minimize back-scattering of neutrons by the vault walls. The detector used here was the same $17 \mathrm{c} . \mathrm{c}$. $\mathrm{Ge}(\mathrm{Li})$ crystal previously discussed. Two different $\mathrm{Cf}-252$ neutron sources were available. One is a point source, which is mounted on the top of a $1 / 4$ in. diameter, 2 in. long aluminum rod. The other is mounted in a $7 / 8$ in. diameter, $1-1 / 2$ in. long lead cylinder. Both soürces gave the same spectra at $691.4 \mathrm{KeV}$, except for the accompanying fission gamma-ray background in that energy region. Furthermore it was found that the spectra at $691.4 \mathrm{KeV}$ are not appreciably affected by varying the direction of the incident neutron beam relative to the $\mathrm{Ge}(\mathrm{Li})$ crystal axis. In any case, after subtracting the background from the broadened spectrum the spectrum has the shape shown in Fig. 5-2. Figure 5-2 is the broadened spectrum at $691.4 \mathrm{KeV}$ produced by bombardment of the $\mathrm{Ge}(\mathrm{Li})$ crystal by the Cf-252 fission neutron source. In the present work the energy resolution, the full width at the half-maximum (FWHM), is eight channels. The broadening by the $\mathrm{Ge}(\mathrm{L} i)$ recoil is shown to be more than 150 channels in Fig. 5-2. Since the energy per channel of this run is $0.425 \mathrm{KeV} / \mathrm{CH}$, the broadening is 


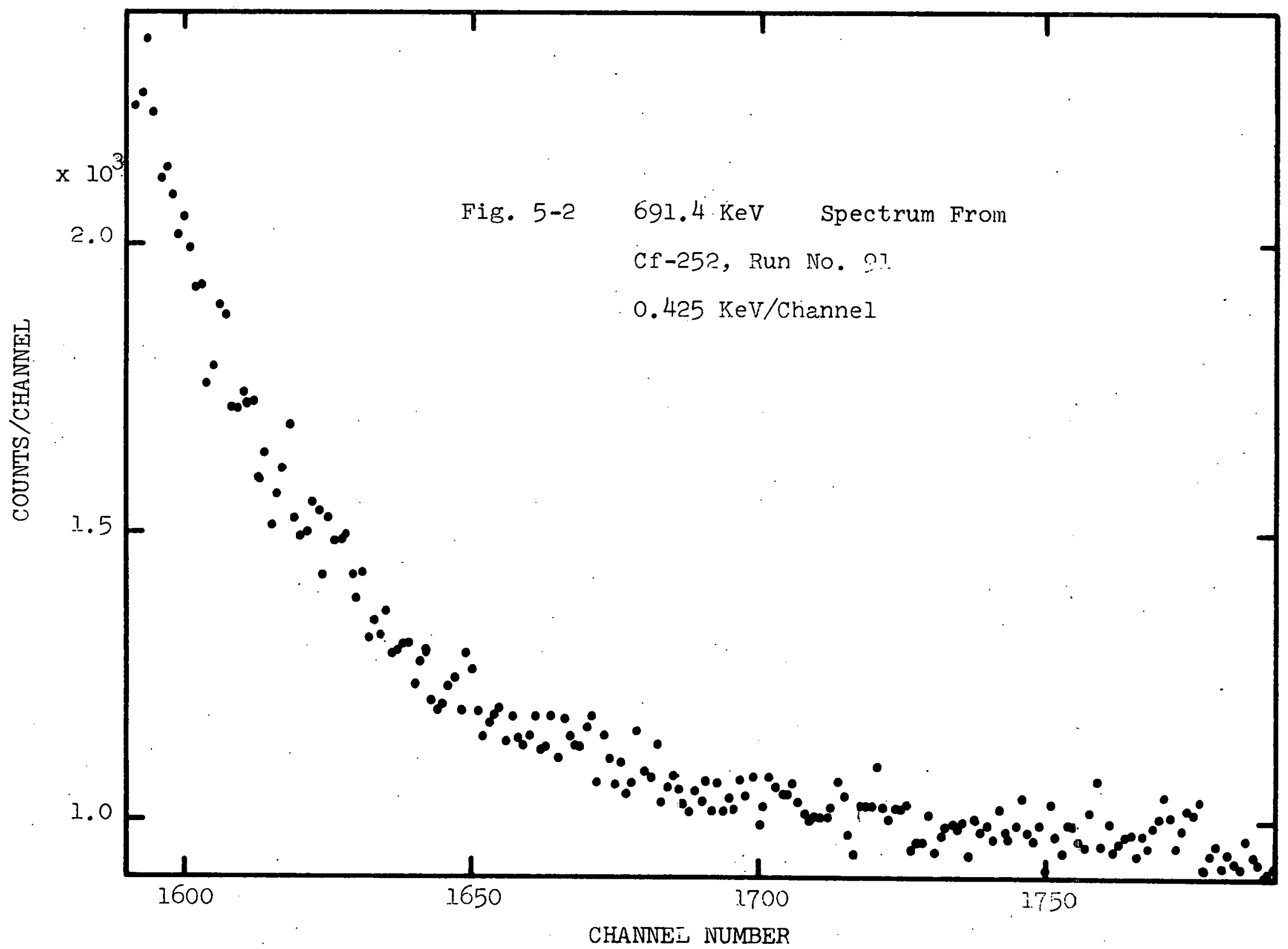


more than $64 \mathrm{KeV}$ on the higher energy side, with better than $4 \mathrm{KeV}$ energy resolution.

Figure 5-3 shows the neutron energy spectra of $\mathrm{Cf}-252$; the dots show the theoretical, or "true" Cf-252 fission neutron energy spectrum and the crosses show the deduced inelastic collision density spectrum, $\sigma_{\text {in }} \phi$, from the measured broadened gamma spectrum at $691.4 \mathrm{KeV}$. Since the inelastic scattering cross-section data are not well established, it is better to include the cross-section in the so-called response matrix. The response correction matrix is calculated from these two independent spectra. Figure 5-4 shows a broadened gamma spectrum at $691.4 \mathrm{KeV}$ produced by neutrons leaking from Blanket No. 2. This original continuous broadened spectrum from Blanket No. 2 is analyzed with the help of the correction matrix, obtained using the $\mathrm{Cf}-252$ standard neutron source. The analyzed neutron spectra of Runs No. 107 and 106 from Blanket No. 2 are shown in Figs. 5-5 and 5-6, respectively. This analysis is done by a computer program SPECT (see Appendix F). Since the internal conversion transition is a threshold reaction, one can only obtain the neutron spectrum above the threshold energy of the reaction. Both Figs. 5-5 and 5-6 exhibit large depressions in the neutron flux at $2.2 \mathrm{MeV}$ and $3 \mathrm{MeV}$. These depressions are 


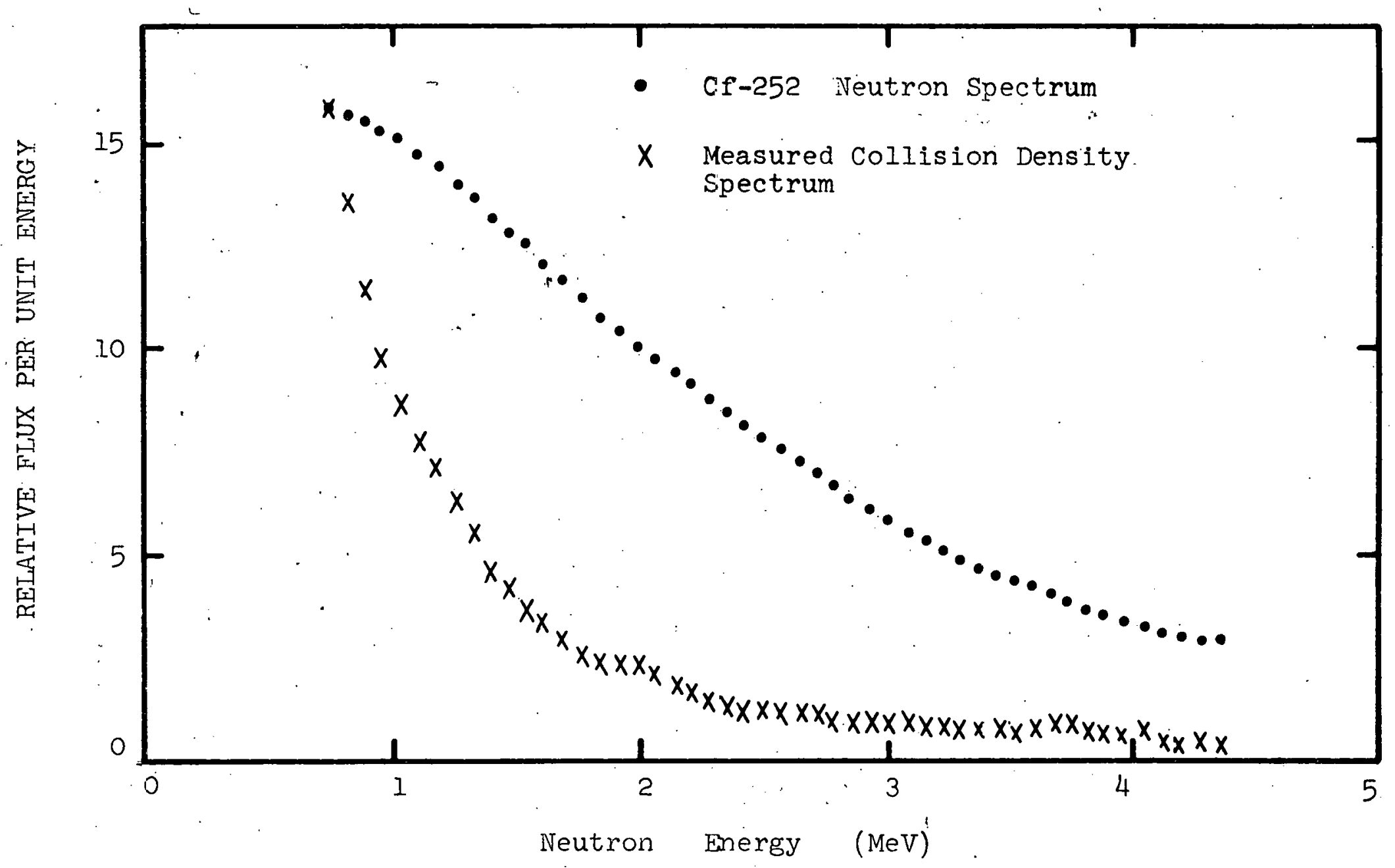

Fig. 5-3 Comparison of the Cf-252 Neutron Spectrum with the

Measured Collision Density Spectrum. 


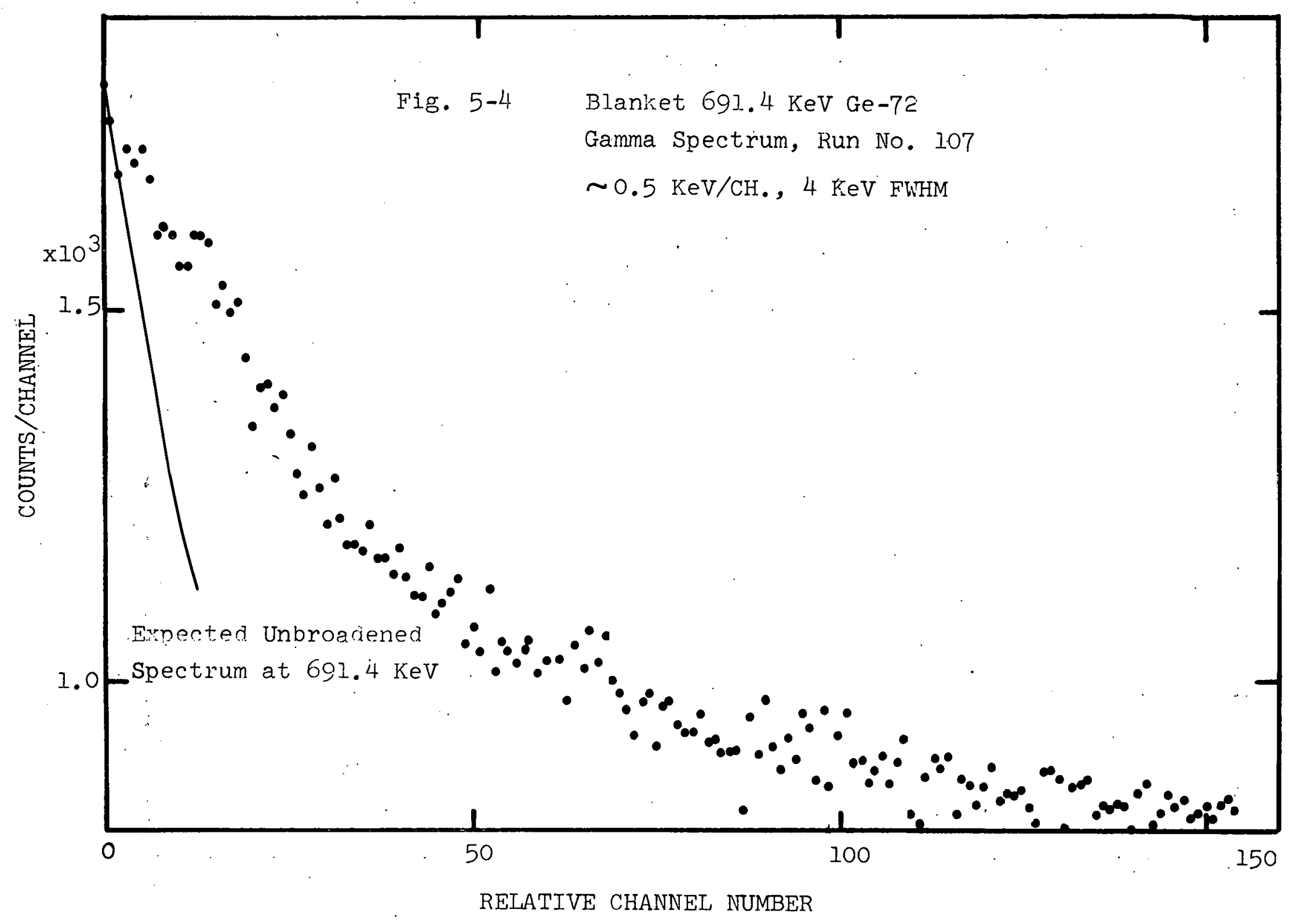




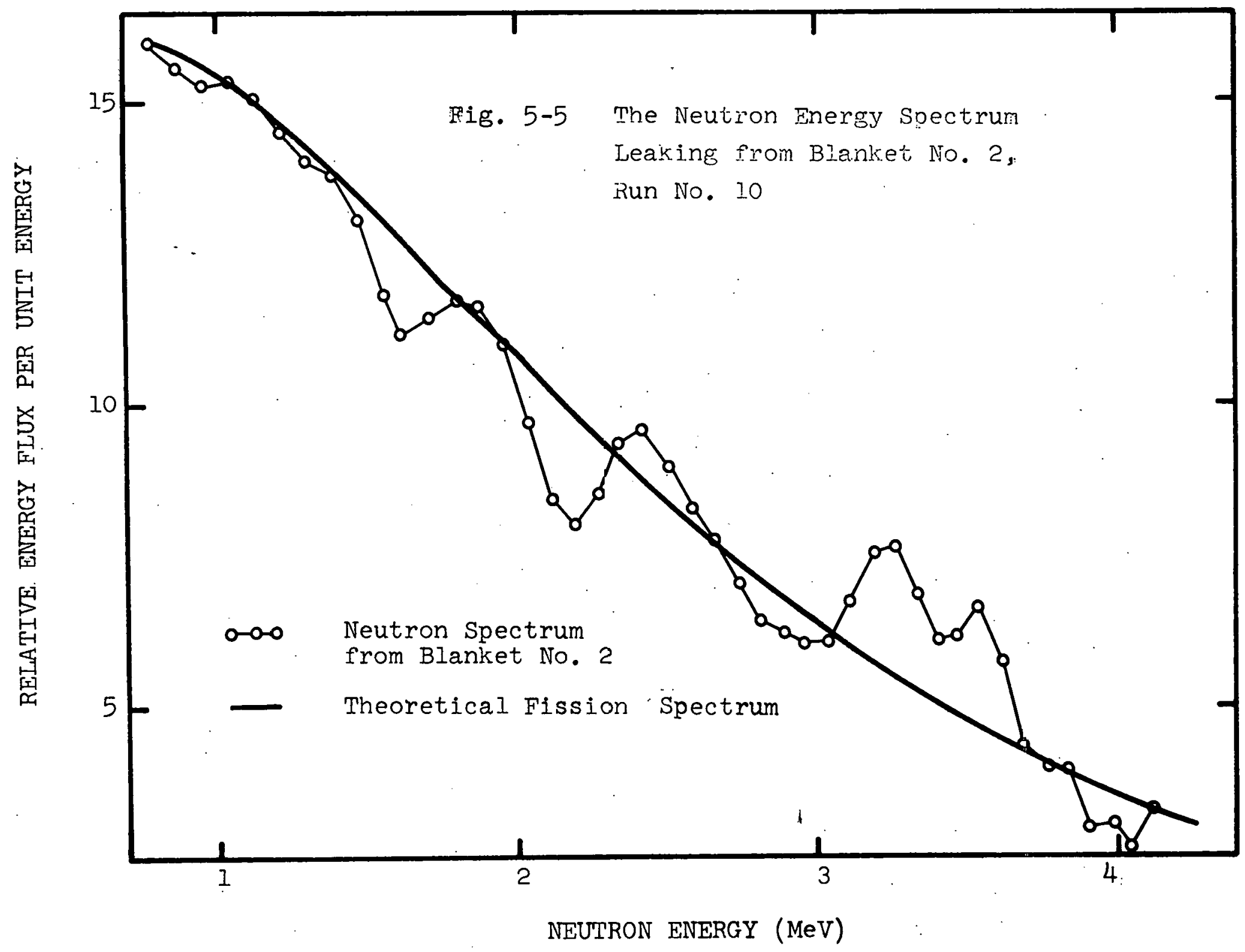




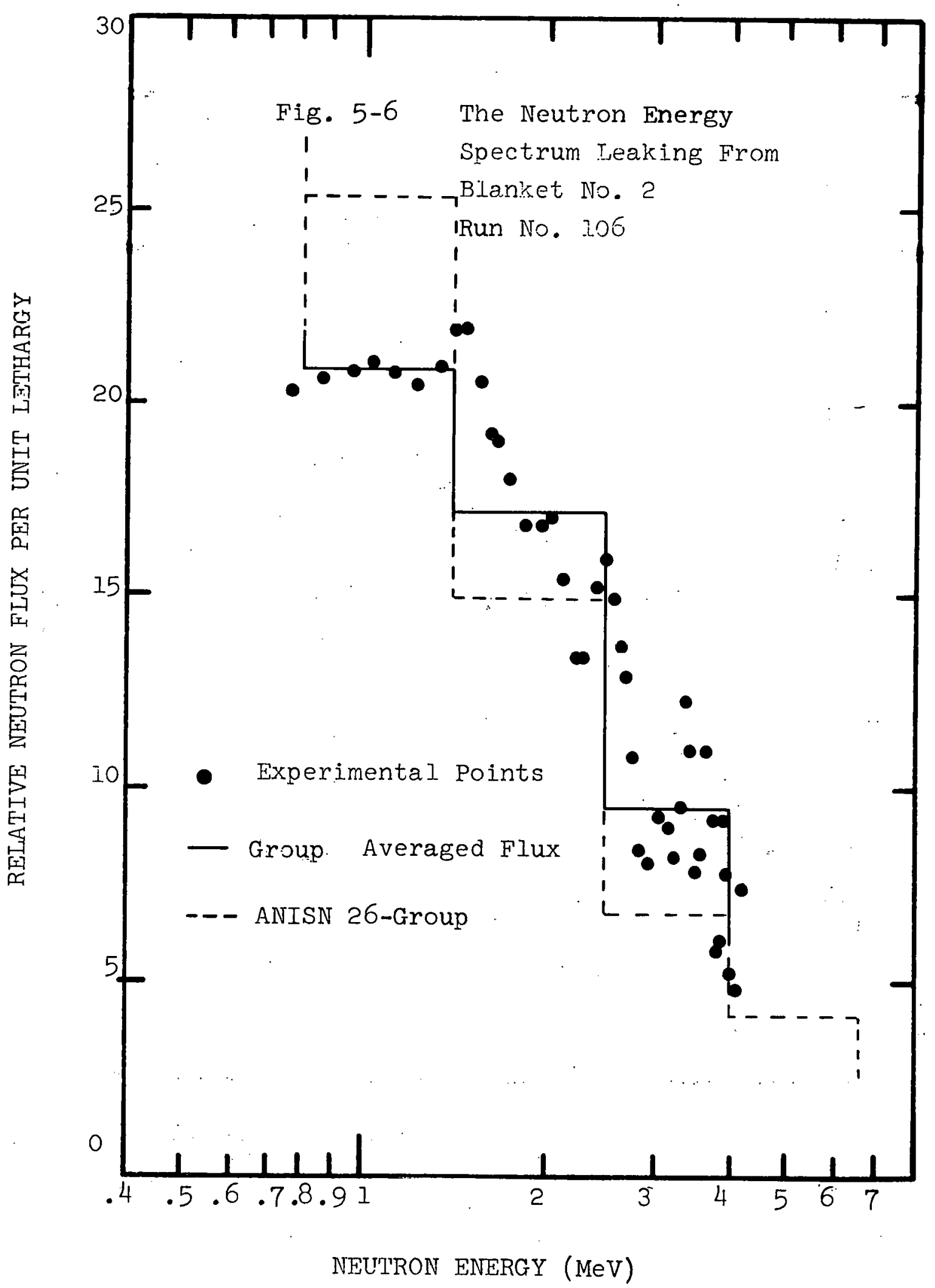


also observed in the neutron spectrum in $\mathrm{ZPR}-3$ Assembly 48 deduced by both $\mathrm{MC}^{2}$ calculation and protonrecoil measurement at ANL (Y2). This is attributable to high energy resonances a.t these energies. Oxygen, chromium and sodium all have large scattering resonances in this energy range. The spectrum from the blanket is compared with the typical fission neutron spectrum in Fig. 5-5 and with the ANISN 26 group flux in Fig. 5-6, respectively.

Methods are suggested to cover the low energy section of the neutron energy spectrum in the next section. 5-4 Some Other Methods for Extracting Neutron Spectra from Gamma-Ray Spectra

There are other possible approaches to unfolding neutron spectra from gamma-ray spectra. For example, Forsberg exploited the fact that the capture gamma-ray yield changes according to the energy of the incident neutrons (F3). Here two additional methods for obtaining neutron energy spectra using gamma-ray spectroscopy will be discussed. 5-4-1 Energy Shift Method

The analytical relationship between the $Q$-value, and the kinetic energy of the incident and compound particle, as measured in the laboratory system, can be deduced. We 
consider here the non-relativistic case, in which the kinetic energy, $E$, of the incident and compound particles is small compared with the rest mass-energy $\mathrm{MC}^{2}$. As an example, consider a neutron of energy $F_{n}$ and mass $\mathbb{N}_{n}$ hitting a target nucleus and being captured by the target, releasing a prompt capture gamma-ray. Conservation laws for nuclear reactions give the following equation (E7):

$$
E \gamma=X \pm \sqrt{X^{2}-Y}
$$

where

$$
\begin{aligned}
& x:=c \sqrt{2 M_{n} E_{n}} \cos \theta-M_{c} C^{2}, \\
& Y=2 \cdot\left[\left(M_{n}-M_{c}\right) C^{2} \cdot E_{n}-Q M_{c} C^{2}\right], \\
& E \gamma, E_{n} \text { - are the energies of the prompt capture } \\
& \text { gamma-rays, and the incident neutrons, } \\
& \text { respectively, } \\
& M_{n}, M_{c} \text { - are the masses of a neutron and the } \\
& \text { compoun'd nucleus formed, respectively, } \\
& \text { Q - is the Q-value of the reaction, } \\
& \text { C - is the velocity of light, } \\
& \theta \text { - is the trajectory angle of the compound } \\
& \text { nucleus after the neutron is captured. }
\end{aligned}
$$


The energetically possible solutions are those for which $\mathrm{E} \gamma$ is real and positive. Two special cases of interest will be examined.

Case I: Thermal neutron bombardment, i.e., $E_{n} \approx 0$.

$$
E \gamma=M_{c} C^{2}\left(\sqrt{1+\frac{2 Q}{M_{c} C^{2}}}-1\right)
$$

Or if we expand in a power series, the gamma-ray energy can be approximated, as expected, by:

$$
\begin{aligned}
E \gamma & =M_{C} C^{2}\left(\frac{Q}{M_{c} C^{2}}-\frac{1}{2} \times \frac{Q^{2}}{\left(M_{c} C^{2}\right)}+\cdots\right) \\
& \approx Q
\end{aligned}
$$

Hence in thermal neutron capture reactions, one can see a gamma-ray, the energy of which corresponds to $Q$, as well as cascades of gamma-rays. In the special case of hydrogen the Q-value is $2.223 \mathrm{MeV}$, which can be seen prominently in every thermal prompt capture gamma-ray spectrum involving hydrogeneous targets.

Case II: The target is hydrogen and the detector is placed at a $90^{\circ}$ angle to the direction of the neutron beam, i.e., $M_{c}=2 M_{n}$ and $\theta= \pm 90^{\circ}$,

$$
E \gamma=\left(Q+\frac{E n}{2}\right)-\frac{I}{4} \frac{\left(Q+E_{n} / 2\right)^{2}}{M_{n} C^{2}}+\ldots .
$$


Hence the shift of the gamma-ray energy from the thermal capture line at $2.223 \mathrm{MeV}$ depends on the energy of the incident neutrons. This effect may make it possible to deduce the incident neutron energy spectrum. However this method has several disadvantages. The first one is that the capture cross-section of hydrogen decreases very rapidly (roughly as $\mathrm{E}^{-1 / 2}$ ) as the energy of the neutrons increases. The second disadvantage is that the possibility of scattering is much higher than that of capture. Thus for thick targets moderation and subsequent capture is actually preferred to initial capture. (see calculation in Appendix I). In order to avoid this problem a very thin target is called for, which, again because of the small $\sigma_{a}$, results in very. low detection efficiency. 5-4-2. Prompt Activation Method

A very well-known method for: analys is of neutron energy spectra is that of foil activation analysis. In this method one can choose a number of special elements; irradiate them for a certain time, and count the decay gamma-rays after a certain cooling period. A direct analogy to this approach is possible, using prompt instead of decay gamma-rays. In fact, rather than target foils one can use gamma-rays given off by the normal constituents of the assembly itself. Prompt gamma-rays from a typical 
multiplying assembly include the inelastic gamma-rays, the prompt capture and fission gamma-rays, and the very short lived decay gamma-rays. Applications involving inela.sti.is gamma-rays appear very enoouraging.

Moșt cross-sections for higher energy neutrons are very small, except for the scattering crosis-section. Further, in the case of prompt capture gamma-rays the yield changes according to the energy of the incident neutrons. Because these varying yields are not well known, this complication will lead one to use inelastic gamma-rays for prompt activation analysis of the present sort. Further production of gamma-rays by inelastic scattering increases as the neutron energy increases. Furthermore, the cross-section for gamma-ray production by inelastic scattering is well known for many elements ( $R I$ ) such as $\mathrm{F}, \mathrm{Ta}, \mathrm{Fe}, \mathrm{Nb}, \mathrm{W}, \mathrm{Sc}$, etc.

The total gamma-ray intensity of a peak of interest due to inelastic scattering can be expressed by:

$$
I_{i}=\epsilon_{i} \int \Sigma_{i}(E) \varnothing(E) d E ;
$$

where

$I_{i}$ - is the total intensity of the inelastic gammaray peak i, 
$\epsilon_{i}$ - is the total efficiency of the detection system for the gamma-ray peak i,

$\Sigma_{i}(E)$ - is the macroscopic inelastic gamma-ray producing cross-section for the gamma-ray peak $i$ at the neutron energy $E$, and

$\phi(E)$ - is the incident neutron flux having energy $E$. Many approaches are appropriate for solving integral equations of the type Eq. 5-37. Here one of the simpler treatments will be introduced. The unknown flux $\phi(\mathrm{E})$ may be expanded in a series of known functions as follows :

$$
\not(E)=\sum_{j} a_{j} u_{j}(E)
$$

where

$$
\begin{aligned}
& a_{j} \text { - are unknown coefficients, and } \\
& u_{j}(E) \text { - appropriate known trial functions. }
\end{aligned}
$$

Substituting Eq. 5-38 into Eq. 5-37 gives:

$$
I_{i}=\epsilon_{i} \sum_{j} a_{j} \Sigma_{i j},
$$

where

$$
\Sigma_{i j}=\int_{i} \Sigma_{i}(E) u_{j}(E) d E .
$$


The matrix elements, $\dot{\Sigma}_{i j}$, can be computed and Eq. 5-40 becomes a well known form of matrix equation if one can choose $\mathrm{N}$ well-resolved inelastic gamma-ray peaks.

$$
[\perp]=[\Sigma] \cdot[A]
$$

where

$$
\begin{aligned}
{[I]=} & \operatorname{col}\left\{I_{1} /{ }_{1}, I_{2} / 2, \ldots, I_{N} /{ }_{N}\right\} \\
{[\Sigma]=} & \text { NXN matrix, the elements of which are - } \\
& \text { defined in Eq. } 5-43, \text { and } \\
{[A]=} & \operatorname{col}\left\{a_{1}, a_{2}, \ldots, a_{N}\right\} .
\end{aligned}
$$

Therefore the spectrum coefficient vector $[A]$ is given by :

$$
[A]=[\Sigma]^{-1}[I]
$$

Equation $5-42$ can be solved in so far as the matrix $[\Sigma]$ is not singular, which can be accomplished by proper choice of the peaks. An advantage of this procedure is that one can use a large number of peaks, since some materials such as $\mathrm{Ta}, \mathrm{Nb}$, Sc have almost ten prominent inelastic gamma-ray peaks (RI). Furthermore thermal neutrons no longer interfere with the experiment. However several disadvantages are incurred. One is that we cannot get any information on neutron spectra lower than about $100 \mathrm{KeV}$ because inelastic scattering is a 
140

threshold reaction. Another disadvantage is that the gamma-ray production cross -section is not well determined which may introduce large errors in the unfolding unless methods using Cf-252 standards are again developed. Further, gamma-ray background problems are often severe. because inelastic gamma-rays are typically of lower energy than prompt capture gamma-rays or even some decay gamma-rays. 


\section{Chapter VI}

\section{GAMMA-RAY DOSIMETRY}

\section{6-1 Introduction}

The radiation environment of a reactor is not exclusively composed of neutrons; gamma-rays are also an important component. The intensity and energy spectrum of the gamma-rays in the blanket are of practical interest in such areas as gamma-ray heating, shielding, and radiation damage.

In most experiments with $G e(L i)$ detectors for gamma-ray measurements, the dominance of the Compton recoil continuum in the deteclurs is regarded a very unwanted and troublesome complication. R. Gold (G5), however, showed how this background signal can be exploited for continuous gamma-ray spectroscopy. He used the well-defined integral equation, which relates the Compton distribution of the recoil electrons and the incoming continuous gamma-ray. spectrum, and solved the integral equation numerically using an iterative unfolding method for response matrices (G7). R. Gold used a small $\mathrm{Si}(\mathrm{Li})$ crystal $\left(200 \mathrm{~mm}^{2}\right.$ area by $5 \mathrm{~mm}$ depletion depth) and focused his major effort on low energy gamma-rays (lower than $1.5 \mathrm{MeV}$ ) in his work. 
For many purposes, such as shielding and radiation damage, high energy gamma-rays are also very important. The present work will cover the high energy region of the gamma-ray spectrum from Blanket No. 2 (up to $10 \mathrm{MeV}$ ). The main objective of the work reported in this chapter is the measurement of the continuous gamma-ray spectrum leaking from Blanket No. 2 .

The continuous gamma-ray spectrum is contributed by not only the gamma-rays produced in the fission process but also the gamma-rays resulting from neutron interactions with the structural materials. Depending on the type of material used, the spectrum of gamma-rays can be considerably enhanced, both in energy and intensity, by the production of inelastic scattering and capture gamma-rays. Thus, when stainless steel was substituted for aluminum as the structural and cladding material in the pool-type ORNL Bulk Shielding Reactor (S4), it was expected that the spectrum of gamma-rays emitted by the core would harden since the gamma-rays above $5 \mathrm{MeV}$ originate primarily from thermal neutron capture in the components of the stainless steel such as $\mathrm{Fe}^{57}$ at $5.91,6.02$, and $7.6 \mathrm{MeV}, \mathrm{Fe}^{58}$ at $10.16 \mathrm{MeV}$, $\mathrm{Cr}^{54}$ at $8.88 \mathrm{MeV}$ and $9.72 \mathrm{MeV}$, and $\mathrm{Ni}^{59}$ at 8.53 and 8.99 MeV. 
We should also point out that the subject gamma spectrum is obtained "free" in every gamma-ray spectrum measured on Blanket No. 2 , just as were the neutron spectra using the $693.5 \mathrm{KeV}$ germanium internal conversion. These two by-products of the gamma-ray spectra suggest the possibility of using $\mathrm{Ge}(\mathrm{Li})$ detectors as simultaneous neutron - gamma spectrometers in LMFBR shielding studies. 6-2 Theory

The measured electron recoil spectrum due to Compton scattering by the incoming gamma-rays to the $\mathrm{Ge}(\mathrm{Li})$ detector is given by:

$$
I(E)=\int_{E^{\prime} \min }^{E^{\prime}} \max K\left(E, E^{\prime}\right) \quad \varnothing\left(E^{\prime}\right) d E^{\prime},
$$

where the function $K\left(E, E^{\prime}\right)$ is customarily called the response function of the detection system, which is the Compton scattering kernel defined by the Klein-Nishina formula in the form (NI):

$$
\begin{aligned}
K\left(E, E^{\prime}\right)= & \frac{\pi r_{0}^{2}}{\left(E^{\prime}-E\right)}\left\{\left(\frac{E}{E^{2}}\right)^{2}+2\left(\frac{E^{\prime}-E}{E^{\prime}}\right)^{2}+\frac{\left(E^{\prime}-E\right)}{E^{\prime 3}} .\right. \\
& {\left.\left[(E-1)^{2}-1\right]\right\}, }
\end{aligned}
$$


where $r_{0}$ is the classical electron radius, and $\phi\left(E^{\prime}\right)$ is the total photon flux at energy $E^{\prime}$.

Equation 6-1 is well-known as a non-singular

Fredholm equation of the first kind. The solution of this kind of integral equation may be treated numerically. It is customary to introduce a matrix formulation using the energy-group approximation. One divides the E-space into $N$ equally spaced energy intervals, whose mid-bin energies are $E_{1}, E_{2},---, E_{N-I}, E_{N}$. For a particular value at $E=E_{i}$, Equation $6-1$ becomes

$$
I_{i}=\int_{E^{\prime} \min }^{E^{\prime}} K\left(E_{i}, E^{\prime}\right) \phi\left(E^{\prime}\right) d E^{\prime},
$$

where

$$
I_{i}=I\left(E_{i}\right)
$$

If one divides the integral term of Equation 6-3 into $N$ small energy intervals, Eq. $6-3$ can be rewritten as:

$$
I_{i}=\sum_{j=1}^{N} \int_{\Delta E^{\prime}{ }_{j}} K\left(E_{i}, E^{\prime}\right) \varnothing\left(E^{\prime}\right) d E^{\prime},
$$

where

$$
\Delta E^{\prime}{ }_{j}=E_{j}^{U^{\prime}}-E_{j}^{L^{\prime}} \text {, and } E_{j}^{U^{\prime}} \text { and } E_{j}^{L^{\prime}} \text { are the upper and }
$$
lower limits bounding the $j$-th bin, $E^{\prime}{ }_{j}$, of $E^{\prime}$-space. 
Using physical constraints imposed by the Compton scattering process $(\mathrm{E}-6)$, one can rewrite Eq. $6-5$ as follows :

$$
I_{i}=\sum_{j} \int_{\Delta E^{\prime} j_{j}} K\left(E_{i}, E^{\prime}\right) \varnothing\left(E^{\prime}\right) d E^{\prime},
$$

where

$$
j=i, i+1, \cdots, N .
$$

If one can assume that the photon flux $\phi$ is a constant over the small region $\Delta E^{\prime}{ }_{j}$, then Eq. $6-6$ can be approximated by :

$$
I_{i}=\sum_{j} K_{i j} \phi_{j}
$$

where

$$
\begin{aligned}
& \dot{I}_{i}=\int_{\Delta E_{i}} I(E) d E / \Delta E_{i}, \\
& K_{i j}=\int_{\Delta E_{j}^{\prime}} K\left(E_{i}, E^{\prime} !\right) d E^{\prime},
\end{aligned}
$$

and $\phi_{j}=\varnothing\left(E_{j}^{\prime}\right)$.

Or in the matrix form, the equation becomes:

$$
[\mathrm{I}]=[\mathrm{K}] \quad[\varnothing]
$$

where

$$
[I]=\operatorname{col}\left\{I_{1}, I_{2}, \ldots . ., I_{N}\right\},
$$




$$
[\phi]=\operatorname{col}\left\{\phi_{1}, \phi_{2}, \ldots, \phi_{N}\right\},
$$

and

$$
[K] \text { is the upper triangular matrix, whose elements }
$$

are

$$
K_{i j}=\int_{\Delta E_{j}^{\prime}} K\left(E_{i}, E^{\prime}\right) d E^{\prime} ; j=i, i+1,--, N_{0}
$$

The upper triangular matrix Eq. 6-11 can be solved without any difficulty. Equation 6-11 may be written by:

$$
\begin{aligned}
& I_{N}=K_{N N} \phi_{N} \\
& I_{N-1}=K_{N-1, N-1} \phi_{N-1}+K_{N-I N} \phi_{N} \\
& \because I_{2}=K_{22} \phi_{2}+K_{23} \phi_{3}+\cdots+K_{2 N} \phi_{N} \\
& I_{1}=K_{1 \cdot 1} . \phi_{1}+K_{12} \phi_{2}+\cdots+K_{1 N} \phi_{N}
\end{aligned}
$$

These equations can be solved exactly in order once all integral terms such as $I_{i}$ and $K_{i j}$ are computed and provided. R. Gold introduced an iterative unfolding method (G7) to solve this matrix equation.

The continuum gamma-ray analysis is performed by a computer code COMPT (Appendix $H$ ) which performs the integrations to form the electron and photon vector array by Simpson's rule (H7), the formulation of the matrix equation and the solution of the upper triangular 
matrix equation using an iterative unfolding method. 6-3 Data Analys is

The electron-recoil spectrum observed with any given detector is not necessarily identical to the Compton continuum. It has been generaliy demonstrated that effects of finite detector size introduce a completely independent unfolding problem (G4). The detector should be chosen as large as possible consistent with requirements of good energy resolution, low noise, and the counting rate. By choosing the largest possible detector, one can obtain higher sensitivity and reduce the complexity of the finite size effect unfolding problem. A 17 c.c. $\mathrm{Ge}(\mathrm{L} i)$ crystal is used in this present measurement. A detector of this size does not give rise to appreciable finite-size effects, since the electron range in the energy region of interest is not comparable with the detector thickness. In fact, for a very high electron energy of $10 \mathrm{MeV}$, the range in germanium is only approximately $8 \mathrm{~mm}$ (EI) Getting rid of the finite-size problem makes the entire process very much simpler. R. Gold suggested an electronic Pulse Shape Discrimination (PSD) method to reduce the finite-size problem (G5). However he used a thin Si(Li) detector, $5 \mathrm{~mm}$ thick by $200 \mathrm{~mm}^{2}$ area. A detector of this size introduces non-negligible finite-size effects, 
especially in the high energy region.

Figure 6-1 shows the observed Compton recoil continuum spectrum from Blanket No. 2, which has been analyzed by GAMANL. GAMANL was used to perform the smoothing of the original gamma-ray spectrum from Blanket No. 2 using Fourier Transforms, and to subtract all analyzed discrete peaks from the spectrum. This output, the analyzed background data of the spectrum, became the input to the computer code COMPT, which performed the analysis of the Compton recoil continuum. gamma-ray spectrum. These background data of a typical gamma-ray spectrum from Blanket No. 2 are given in Appendix $\mathrm{H}$.

Figure 6-2 shows the gamma-ray continuum leaking from Blanket No. 2 obtained through the computer program COMPT (Appendix H). In Figure 6-2 the component of the spectra due to prompt neutron captures in the blanket structural materials is evident in the peaks appearing above $5 \mathrm{MeV}$. The photions above $5 \cdot \mathrm{MeV}$ originate primarily from prompt neutron capture in the iron, which is the cladding and main structural material in Blanket No. 2 , its reflector, and the surrounding shielding. Two prominent $\mathrm{Fe}^{57}$ lines of almost equal intensity, with energies of 5.921 and 


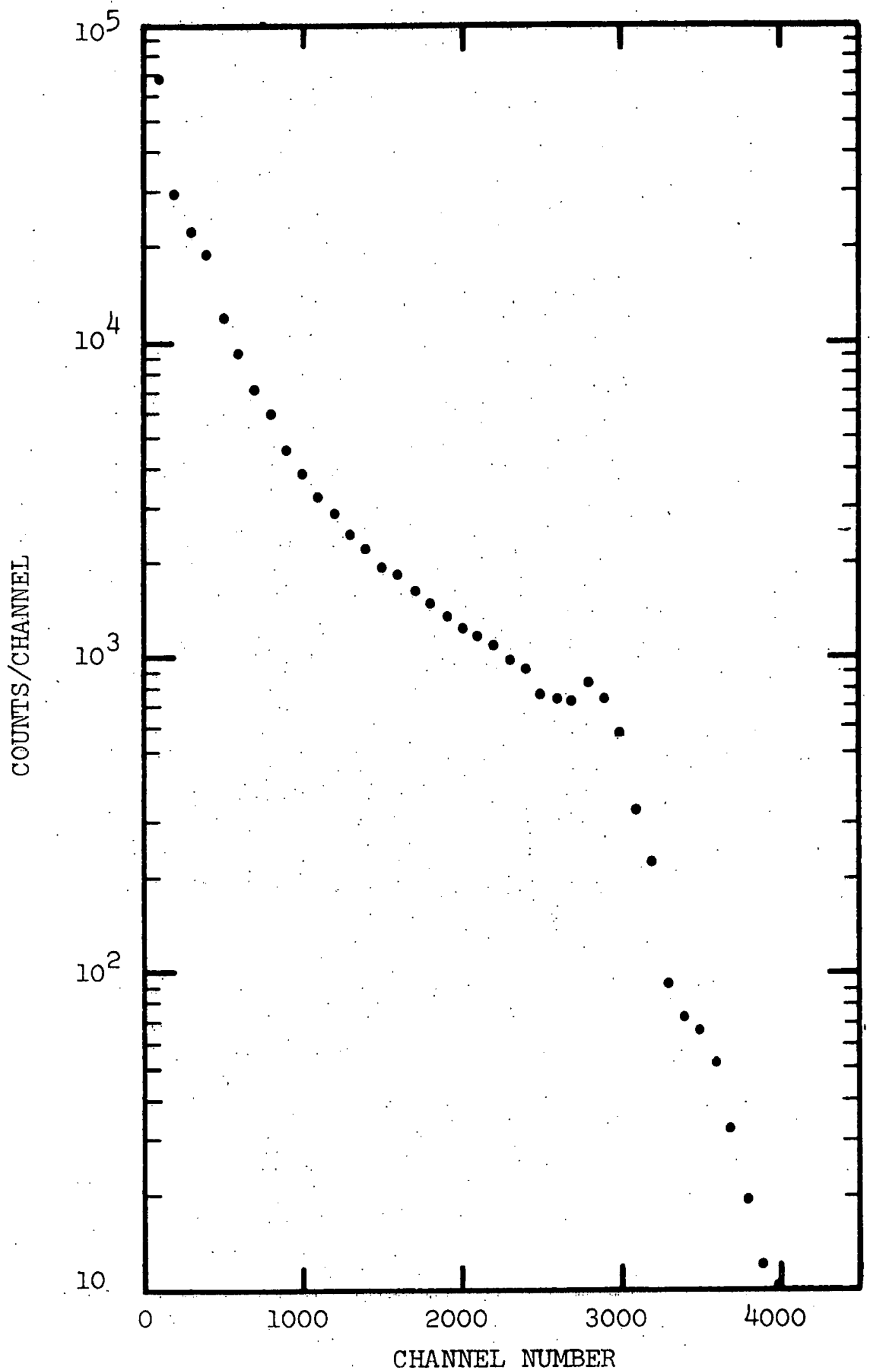

Fig. 6-1 Gamma-Ray Continuum from Blanket No. 2, Run No. 105 


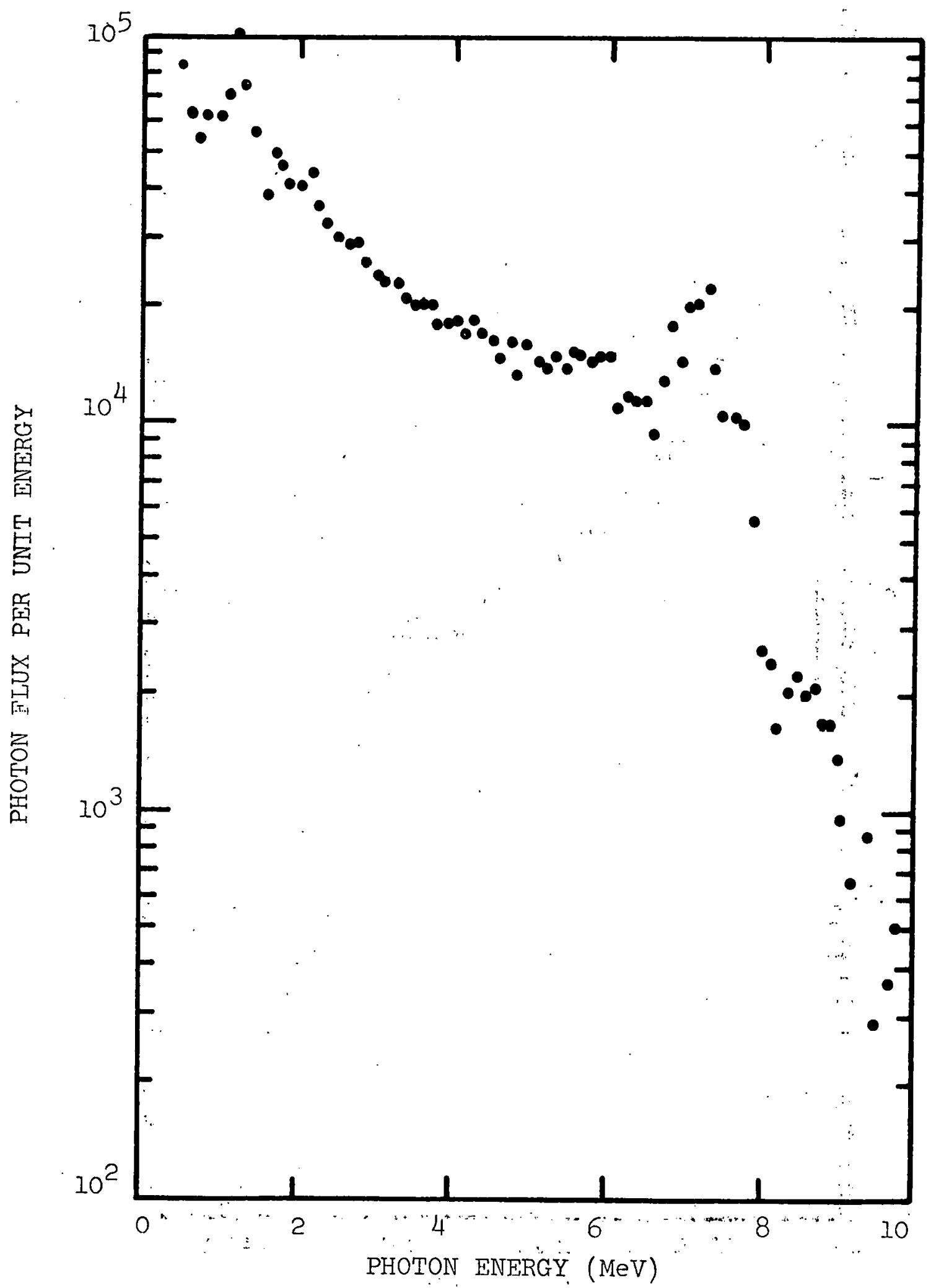

Fig. 6-2 The Gamma-Ray Spectrum Leaking. from Blanket No. 2, Run No. 105 
$6.019 \mathrm{MeV}$, contribute to the spectrum. Other $\mathrm{Fe}^{57}$ capture gamma-rays, at 7.632 and $7.646 \mathrm{MeV}$, appear as the most distinct contribution in the high energy spectrum. These high energy iron prompt capture peaks are responsible for the large continuum flux up to $7.5 \mathrm{MeV}$. Above this energy the flux decreases abruptly. The distribution between 8.5 and $9 \mathrm{MeV}$ is composed of gamma-rays from $\mathrm{Cr}^{54}$ at $8.881 \mathrm{MeV}$, plus a possible weak contribution of gamma-rays from $\mathrm{Fe}^{57}$ at $8.872 \mathrm{MeV}$. Above $9 \mathrm{MeV}$, the major contribution comes from $\mathrm{Cr}^{54}$ at $9.716 \mathrm{MeV}$ and from $\mathrm{Fe}^{58}$ at $10.16 \mathrm{MeV}$. Below $5 \mathrm{MeV}$ the spectrum in Figure 6-2 is apparently contributed by gamma-rays that constitute the expected cuntinua of prompt fission and fission product gamma-rays plus a contribution by neutron capture and inelastic scattering in sodium, chromium, uranium, iron, etc.

The first bump at $1.2 \mathrm{MeV}$ in Figure $6-2$ is due to the high background gamma-ray from $\mathrm{Ar}^{41}$ at. $1.294 \mathrm{MeV}$. The second bump at $7.3 \mathrm{MeV}$ is due to the most distinct peak of iron prompt capture, the $7.64 \mathrm{MeV}$ doublet. The third bump at $8.5 \mathrm{MeV}$ is due to the prompt capture gamma-ray from $\mathrm{Cr}^{54}$ at $8.882 \mathrm{MeV}$. The analys is has been done in a very straight forward manner, neglecting the finite size problem. This finite size effect should not 
be appreciable in this measurement. Most errors introduced in unfolding the spectrum are in the high energy region, and are caused mainly by neglecting the finite-size problem. 
Chapter VII

CONCLUSIONS AND RECOMMENDATIONS

7-1 Conclusions

The present work was a study of the application of gamma-ray spectroscopy to the investigation of fast reactor blankets. This AEC sponsored work was carried out on Blanket No. 2 of the M.I.T. Blanket Test Facility. As a result of this study the following conclusions were reached:

1. Ge(Ii) detectors can be used to determine simultaneously the fast neutron spectrum $\left(E_{n}>.8 \mathrm{MeV}\right)$ and the high energy gamma-ray spectrum. This information can be obtained from a single spectrum from a Ge(Li) detector. The neutron spectrum is obtained from an analysis of broadened Ge lines and the gamma-ray spectrum from an analysis of the Compton distribution. This information about the high energy portion of each spectrum would be particularly useful in shielding studies. 
2. The hydrogen content of materials in a fast system can be accurately determined by prompt activation analysis using the hydrogen capture gamma-ray. This information is particularly. useful since small hydrogen impurities can have important effects on the neutron spectrum in fast facilities.

3. The determination of the reaction rates in various components of the fast facility can in principle be determined by analysis of a combination of capture inelas, tic, and decay gamma-rays. In this study it was found that this method did not give a high degree of accuracy. The accuracy was limited by lack of knowledge of the gamma-ray.yield as a function of neutron energy. Another serious Jjmitation discovered was that no: capture lines from U-238 could be identified in the fast neutron spectrum even though a number can be measured in a thermal neutron spectrum. 
This means that capture gamma-rays from the dominant capture mode in the blanket cannot be detected in measurements of the type carried out in this study. For this reason this methed does not appcar promising for applications of the type investigated. It was shown that the reaction rate in $U-238$ could be determined from the decay gamma-rays of Np-239. However the low energy of these gamma-rays (100-300 $\mathrm{KeV})$ led to vary large self absorption corrections, which are difficult to determine accurately. This comment also applies to the gammarays from inelastic neutron scattering. 7-2 Recommendations.

Based upon the results of this investigation, a number of both specific and general recommendations are suggested:

1. It is suggested that in future work at M.I.T. the possibility of using a long-nose dewar to hold the Ge(Li) crystal closer to the blanket be investigated. This will increase the counting rate of the detecting system and reduce the statistical error. 
2. It is recommended that a study of prompt capture yiejd changes of U-238, sodium, chromium and other IMFBR constituents be made as a function of neutron energy if further development of prompt gamma analys is methods is attempted. It should be noted, however, that this will be a very tedious and expensive task. Similarly, further study of the relationship between the gamma-. ray production and inelastic scattering cross-section would be necessary. In any event it is recommended that successful extraction of U-238 prompt capture gamma lines be deomonstrated before embarking upon the preceding work.

3. Analysis and unfolding of the broadened inelastic scattering spectrum of $\mathrm{Ge}^{74}$ at $595 \mathrm{KeV}$, and comparison with the spectrum unfolded using tie $691.4 \mathrm{KeV}$ spectral line would appear useful. 
4. Development of external target methods to reduce background (especially Fe prompt capture gamma-rays, which cause great problems in all spectra) is suggested.

5. Further study of the energy shift method using low $-Z$ materials for measurement of neutron spectra is probably not warranted based upon its demonstrable low efficiency.

6. Finite size effects of the Ge(Li) crystal (GI) should be investigated in more detail in regard to the Compton recoil continuum analysis. 


\section{REFERENCES}

Bl E. Barrington, A. Pope and J. Story, "The data in the Winfrith Nuclear Data Library", AEEW-R, 351, $5(1964)$

B2 R. E. Bell and L. G. Elliot, "Gamma-Rays from the Reaction $H(n, \gamma)$ D and the Binding Energy of the Deuteron," Phys. Rev., 74, 1552 (1948)

B3 R. E. Bell and L. G. Elliot, "Gamma-Rays from the Reaction $H(n, \gamma)$ D and the Binding Energy of the Deuteron", Phys. Rev., 79, 282 (1950).

B4 T. W. Bonner, "Measurements of Neutron Spectra. from Fission", Nucl. Phys., 23116 (1961)

Cl E. L. Chupp, R. W. Jewell, Jr. and W. John, "Binding Energy of the Deuteron Measured with a Bent Crystal Spectrometer," Phys. Rev., 12l, 234 (1961)

C2 C. Chaseman, K. Jones and R. Rinstein, "Measurement of the Energy Loss of Germanium Atoms to Electrons in Germanium at Energies below $100 \mathrm{KeV}, "$ Phys. Rev. Lettcro, 15, 245 (1965)

C3 C. Chaseman, $K$ Jones, "Band-Gap Effects in the Stopping of Ge 72 Atoms in Germanium, "Phys. Rev. Letters, 21, 1430 (1968)

C4 H. Conde and G. During, "Fission Neutron Spectra", Arkiv for Fysik, 29, 313 (1965)

Dl J. Donohew, "Inelastic Scattering in Fast Reactor Materials", SCD Thes is, MIP Nucl. Eng. Dept. (1970)

El R. D. Evans, "The Atomic Nucleus", McGraw-Hill Book Co. (1955) (Chapt. 21, Sec. 3)

E2 R. D. Evans, "The Atomic Nucleus, " McGraw-Hill Book Co. (1955) (Chapt. 10, Sec. 4)

E3 R. D. Evans, "The Atomic Nucleus," McGraw-Hill Book Co. (1955) (Chapt. 12, Sec. 2)

E4 R. D. Evans, "The Atomic Nucleus", McGraw-Hill Book Co. (1955) (Chapt. 6, Sec. 5) 
E5 R. D. Evans, "The Atomic Nucleus", McGraw-Hill Book Co. (1955) (Chapt. 6, Sec. 4)

E6 R. D. Evans, "The Atomic Nucleus", McGraw-Hill Book Co. (1955) (Chapt. 23, Sec. 2)

E7 R. D. Evans, "The Atomic Nucleus", McGraw-Hill Book Co. (1955) (Chapt. 12, Sec. 2)

Fl I. A. Forbes, J. J. Driscoll, T. J. Thompson,

I. Kaplan and D. D. Lanning, "Design, Construction and Evaluation of a Facility for the Simulation of Fast Reactor Blankets", MIT-4105-2, MITNE-110 (February, 1970)

F2 H. Feshbach and J. Schwinger, "On a Phenomenological Neutron-Proton Interaction", Phys. Rev., 84, 194 (1951)

F3 C. W. Forsberg, "Determination of Neutron Spectra by Prompt Gamma-Ray Spectroscopy", M. S. Thesis, MIT Nucl. Eng. Dept. (June, 1971)

GI R. Gold, "Compton Recoil Continuum Measurements for In-Core Gamma-Ray Spectroscopy", Reactor Phys. Div. Annua 1. Report (July 1, 1967 - June 30, 1968), ANL-7410 PP. 373-377

G2 L. Groshev, et al., "Compendium of Thermal Neutron Capture Gamma-Ray Measurements", Part III for $Z=68$ to $Z=94$, Nucl. Data, Section $A$, Vol. 5, No. 3-4 February 1969)

G3 G. Gordon, J. Harvey and H. Nakahara, "Measuring Fission Spectra with Semiconductor Detectors", Nucleonics, No. 12, 62 (December 1966)

G4 R. Gold and E. Bennett, "Effects of Finite Size in

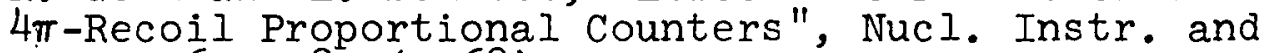
Meth., 63, 285 (1968)

G5. R. Gold, "Compton Recoil Gamma-Ray Spectroscopy", Nucl. Instr. and Meth., 84, 176 (1970)

G6 R. C. Greenwood and W. W. Black, "The Binding Energy of the Deuteron Determined from Measurement of the Hydrogen Neutron Capture Gamma-Ray Energy", Phys. Letters, 21, 702 (1966) 
G7 R. Gold, "An Iterative Unfolding Method for Response Matrix," ANL-6984 (December 1964)

G8 L. Green, "Transmission Measurement of the Cf-252 Fission Neutron Spectrum", Nucl. Sci. and Eng., 37 232-242 (1969)

G9 T. N. E. Greville, "Spline Functions, Interpolation, and Numerical Quadrature," Mathematics Research Center, U. S. Army

HI R. Heath, et al., "Instrumental Requirements for High Resolution Gamma-Ray spectroscopy Using Ge(Li) Detectors", IEEE Trans. Nuc1. Sc., NS-13, 445 (June, 1966)

H2 J. Hamawi and N. Rasmussen, "Investigation of Elemental Analysis Using Neutron-Capture Gamma-Ray Spectra", MITNE-107 (1969)

H3 Y. Hukai, N. Rasmussen and M. Driscoll, "Some Applications of Ge(Li) Gamma-Ray Spectroscopy to Fuel Element Assay", MIT-3944-5, MITNE-113 (April 1970)

H4 'T'. Harper, Jr. and N. Rasmussen, "Determination of Thermal Neutron Capture Gamma Yields", MITNE-104 (July 1969)

H5 R. Heath, "The Potential of High Resolution Gamma Ray Spectrometry for the Assay of Irradiated Reactor Fuel", WASH-1076, 115 (1967)

H6 A. Horsley, "Neutron Cross Sections of the Proton in the Energy Range 0.0001 eV-20 MeV", AWRE-0-23/65 (1965)

H7 F. Hildebrand, "Methods of Applied Mathematics," 2nd Edition Prentice Hall (Chapt. 3, sec. 15)

H8 F. Hildebrand, "Introduction to Numerical Analysis", McGraw Hill (Chapt. 7, sec. 2)

Kl A. H. Kazi, N. Rasmussen and H. Mark, "Measurement of the Deuteron Binding Energy using a Bent-Crystal Spectrograph", Phys. Rev., 123, 1310 (1961) 
K2 J. W. Knowles, "Diffraction Angle Measurements Bearing on the Deuteron Binding Evergy and on the $\mathrm{X}$-Unit-to-Milliangstrom Cnnversion Constant", Can. J. Phys., 40, 257 (1962)

LI "LMFBR Blanket Physics Project Progress Report No. I", MITNE-116, MIT-4105-3 (June 1970)

L2 C. Lederer, J. Hollander and I. Perman, "Table of Isotopes", 6th Ed., John Wiley and Sons, Inc. (1967)

L3 J. Lindhard, V. Nielsen, M. Scharff and P. Thomson, "Integra.1 Equations Governing Radiation Effects", $\mathrm{Kgl}$. Danske Videnskab. Selskab., 33, No. 10 (1963)

L4 M. M. Leving, "Report from Atomic Energy Establishment, Winfrith," Issued by B.N.L. (February 1970)

L5 T. C. Leung; M. J. Driscoll, I. Kaplan and D. D. Lanning, "Neutronics of a LMFBR Blanket Mock-Up", COO-3060-1 MITNE-127, to be issued

MI J. E. Monahan, S. Raboy and C. C. Trail, "Measurement of the Energy of the Gammia. Radiation from Neutron Capture by Hydrogen", Nuc 1. Phys., 24, 400 (1961)

M2 H. T. Motz, R. E. Carter and P.. C. Fisher, "GammaRay Energy Measurements with a Compton Spectrometer", Bu.l.1. Amer. Phys. Soc., 4, 477 (1959)

M3 J. Meadows, "Cf-252 Fission Neutron Spectrum from 0.003 to $15.0 \mathrm{MeV}^{\prime \prime}$, Phys. Rev., 157, 1076 (1967)

N1 A. Nelms, "Graphs of the Compton Energy-Angle Relationship and the Klein-Nishina Formula from $10 \mathrm{KeV}$ to $500 \mathrm{KeV}$," NBS Circular 542 (1953)

Ol V. Orphan and N. Rasmussen, "Study of Thermal Neutron Capture Gamma-Rays Using a Ge(Li) Spectrometer", MITNE-80 (January 1967)

Q2 G. Odette, "Measurement of the Fast Neutron Radiation Hardening Energy Response Function in Iron", ScD Thesis, MIT Nucl. Eng. Dept. (September 1970)

03 N. Ortiz, I. C. Rickard, M. J. Driscoll and N. C. Rasmussen, "Instrumental Methods for Neutron Spectroscopy in LMFBR Applications", COO-3060-3, MITNE-129, to be issued. 
Pl P. Palmedo, H. Ludewig and A. Aronson, "Effect

of Hydrogen Content of Graphite in Fast Critical.

Experiments", ANS Trans., Vol. 13, No.'1, P.. 253 (June 1.970)

RI. V. Rogers, "Neutron Inelastic Scattering Crosssections", PhD Thesis, MIT Nucl. Eng. Dept.

(February 1969).

R2 N. Rasmussen, T. Harper, Jr. and 'T. Inouye, "GAMANL, A Computer Program Applying Fourier Transforms to the Analysis of Gamma Spectra", MIT-3944-2, MITNE-97 (August 1968)

R3 N. Rasmussen, Y. Hukai, T. Inouye and V. Orphan, "Thermal Neutron Capture Gamma-Ray spectra of the Elements", MITNE-85 (January 1969)

R4. "Reactor Physics Constants," ANL-5800, 2nd Ed., U. S. Government Printing Office (July 1963)

Sl J. A. Sovka and N. C. Rasmussen, "Nondestructive Analysis of Irradiated MITR Fuel by Gamma-Ray Spectroscopy", AFCRL-65-787, MITNE-64 (October 1965)

S2 J. Stehn, M. Goldberg, B. Magurno and R. Wienerchasman, BNL-325 (3rd Ed., Supplement No. 2) Vol. I (1964)

S3 A. B. Smith, P. R. Fields and J. H. Roberts, "Spontaneous Fission Neutron Spectrum of Cf-252," Phys. Rev., 108, 411 (1957)

S4 E. G. Silver and J. Lewin, "Safeguard Report for a Stainless-Steel Reactor for the BSF (BSR-I)", ORNI-2470 (July 1958)

TI M. Trammel and $W$. Henninger, "Nuclear Data Library for the Fission Product Program", Astronuclear Lab., Westinghouse, WANL-TME-574 (November 1966)

WI V. Weisskopf, "Statistics and Nuclear Reactions", Phys. Rev. 52, 295 (1937)

Yl K. Yost, J. White and C. Fu, "Neutron Energy Dependent Capture Gamma-Ray Yields for U-238 and Ta-18I," ANS Trans. (Winter 1970) 866, Vol. 13, No..2

Y2́ T. J. Yule and E. F. Bennett, "Measured Neutron Spectra in a Number of Uranium and Plutonium-Fueled Reactor Assemblies", Nucl. Science and Eng. 46, 236-243 (1971) 
Appendix A

CALCULATION OF BLANKET AXIAL NEUTRON FLUX

Application of I-group diffusion theory gives the following equation:

$$
D \nabla^{2} \phi-\left(\Sigma_{a}-\nu \Sigma_{f}\right) \varnothing=0
$$

or

$$
\nabla^{2} \phi-\mathrm{K}^{2} \phi=0
$$

where

$$
\mathrm{K}^{2}=\frac{\Sigma_{a}-\nu \Sigma_{f}}{D}
$$

Reference $L 5$ shows that the neutron flux in the blanket is well represented by the solution of the diffusion equation:

$$
\varnothing(x, y, z, E)=\cos \left(\frac{\pi z}{A}\right) \cos \left(\frac{\pi y}{B}\right) \varnothing(E, x) \cdot(A-4)
$$

Assuming the separability of energy and position, $\phi(E, X)=\varnothing(E) \psi(x)$; the l-group axial neutron flux is further assumed to be in the form:

$$
\psi(x) \sim e^{-\Gamma x}
$$

(than effectively infinite medium is assumed) A semi-log plot of the total neutron flux in Blanket No. 2 calculated by the ANISN code in the S8 option using 26-groups is shows in Fig. A-1. As can be seen, the simple one-group 


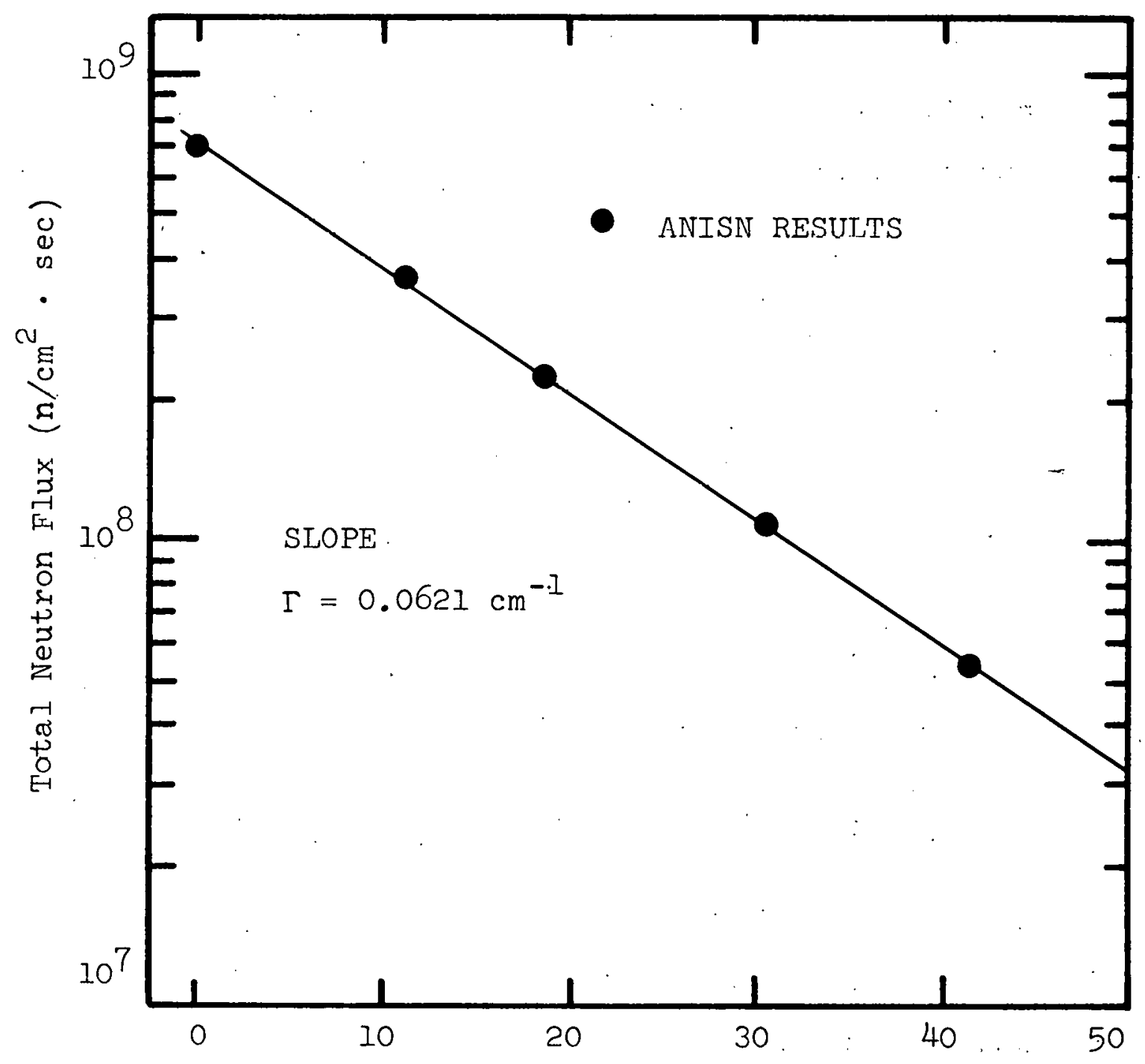

Fig. A-l Total Axial Neutron Flux of Blanket No. 2 Calculated by S8, 26-Group ANISN vs. Distance into Blanket 
model is a quite good representation of the multigroup results Therefore the one dimensional neutron flux across Blanket No. 2 is given by the following equation:

$$
\psi(x)=40 e^{-\Gamma x}
$$

where

$$
\psi_{0}=7.06049 \times 10^{8} \mathrm{n} / \mathrm{sec} \cdot \mathrm{CM}^{2},
$$

and

$$
\Gamma=0.0621 \cdot \mathrm{CM}^{-1}
$$

The average neutron flux over the blanket is:

$$
\begin{aligned}
\overline{4} & =\int_{0}^{x_{B}} \psi_{0} e^{-\Gamma x} d x / x_{B} \\
& =2.72 \times 10^{8} \mathrm{n} / \mathrm{sec} . \mathrm{CM}^{2}
\end{aligned}
$$

The normalized neutron flux is:

$$
\begin{aligned}
\Psi(x) & =4(x) / \int_{0}^{x_{B}} 4(x) d x \\
& =0.0662 e^{-0.0621 x}
\end{aligned}
$$

The normalized flux distribution $\psi(x)$ of Eq. A-Il across Blanket No. 2 is used in the text whenever the axial flux variation needs to be taken into account. 
Appendix B

MEASUREMENTS OF GAMMA-RAY LINEAR

ATTENUATION COEFFICIENTS

At the outset it should be noted that the gamma ray attenuation data experimentally determined here did not agree with the established data ( $R 4)$. In particular, the attenuation coefficients of uranium for low energy gamma-rays are not well resolved yet. Table B-I shows the attenuation coefficients of uranium for some discrete gamma-ray energies.

Three gamma-ray sources were used in these measurements. For high energy gamma-rays ( $1-1.5 \mathrm{MeV}$ ) $\mathrm{Co}^{60}$ and $\mathrm{Na}^{22}$ standard sources were used and for low energy gamma-rays highly depleted uranium foils (18 ppm $\mathrm{U}-235$ ) having a total weight of $0.61 \mathrm{gms}$, were irradiated to produce a 2.35 day half-life $\mathrm{Np}-239$ gamma-ray source. Varying the thickness of uranium attenuators, a series of measurements were done with each gamma source. The thickness of uranium attenuators was varied by combination of .005 inch thick and .02 inch thick uranium foils. The same $17 \mathrm{c} . \mathrm{c}$. crystal was used as a Ge(Li) detector for this purpose. Output data for each gamma-source were fitted by the linear least square method in semilog rectangular coordinates using a subroutine LILSQ 
TABLE B-1

\section{GAMMA-RAY LINEAR ATTENUATION \\ COEFFICIENTS OF URANIUM}

\begin{tabular}{cccc}
\hline \multirow{2}{*}{$\begin{array}{c}\text { Gamma } \\
\text { Sources }\end{array}$} & $\begin{array}{c}\text { Energy } \\
(\mathrm{Kev})\end{array}$ & $\begin{array}{c}\text { Linear Attenuation Coeff. } \\
\left(\mathrm{cm}^{-1}\right)\end{array}$ \\
\cline { 3 - 4 } Np-239 & 209.8 & 25.5 & Referent \\
Work & & $20.6-$ \\
& 228.2 & 20.6 & 18.1 \\
& 277.6 & 13.0 & 8.75 \\
$\mathrm{Co-60}$ & 315.9 & 10.4 & 7.88 \\
& 334.3 & 8.51 & 7.21 \\
$\mathrm{Na}-22$ & 1173.0 & 1.29 & 1.23 \\
$\mathrm{Na}-24$ & 1332.5 & 1.04 & 1.11 \\
\hline
\end{tabular}

* Reference: "Reactor Physics Constants", ANL-5800, Table 8-10

* Extrapolated by three measured values from $\mathrm{Co}^{60}$ and $\mathrm{Na}^{22}$. 
(Appendix D). Table B-2 shows the measured gamma-ray linear attenuation data for varying thicknesses of $\mathrm{Na}_{2} \mathrm{CrO}_{4}$, a major constituent of Blanket No. 2 , and the attenuation data of iron from the literature. 
TABLE B-2

GAMMA-RAY LINEAR ATTENUATION COEFFICIENTS

OF $\mathrm{Na}_{2} \mathrm{Cr} \mathrm{O}_{4}$ and $\mathrm{Fe}$

\begin{tabular}{ccc}
\hline ENERGY(KeV) & LINEAR ATTENUATION & COEFFICIENTS $\left(\mathrm{cm}^{-1}\right)$ \\
\hline $\begin{array}{c}\mathrm{Na}_{2} \mathrm{CrO}_{4} \\
(\text { Measured })\end{array}$ & $\begin{array}{c}\text { Fe } \\
\text { (Reference)* }\end{array}$ \\
209.8 & 0.207 & 1.060 \\
228.2 & 0.204 & 1.014 \\
277.6 & 0.202 & 0.890 \\
315.9 & 0.199 & 0.815 \\
334.3 & 0.197 & 0.795 \\
1173.0 & 0.126 & 0.433 \\
1332.5 & 0.121 & 0.414 \\
1274.5 & 0.122 & 0.405 \\
1368.5 & $0.120 * *$ & 0.401
\end{tabular}

* Reference: "Reactor Physics Constants", ANL-5800, Table 8-10

* Extrapolated by three measured values from $\mathrm{Co}^{60}$ and $\mathrm{Na}^{22}$. 


\section{Appendix C}

CALCULATION OF FAST NEUTRON CAPTURE RATE OF HYDROGEN

The fast neutron capture rate of hydrogen is calculated in this appendix, and compared with that of lower-energy neutron capture. In the reaction with fast incident neutrons the probability of incident neutrons undergoing capture by a sample is

$$
\mathrm{Pi}=\frac{\Sigma_{a}^{\circ}}{\Sigma_{a}^{0}+\Sigma_{s}^{0}},
$$

or

$$
\mathrm{Pi} \approx \frac{\Sigma_{\mathrm{a}}^{\circ}}{\Sigma_{\mathrm{s}}^{0}},
$$

since $\Sigma_{\mathrm{a}}^{0} \ll \Sigma_{\mathrm{s}}^{0}$ for fast neutrons. The probability of a scattered neutron undergoing capture on its first flight out of the sample is.

$$
\mathrm{Pi}=\Sigma_{\mathrm{a}}^{*} \bar{l}
$$

where $\Sigma_{a}^{*}$ is the average absorption cross-section of hydrogen for scattered neutrons, and $\bar{l}$ is the mean chord length of escaping neutrons; which is $\bar{l}=4 / 3 \mathrm{R}$ for an infinite cylindrical rod with radius $\mathrm{R}$ for $a$ uniform internal scattering source. Hence the ratio of the probability of capture reactions by scattered lower 
energy neutrons to that of the incident higher energy neutrons is :

$$
R=\frac{\Sigma_{a}^{*} l}{\Sigma_{a}^{0} / \Sigma_{s}^{0}}
$$

or

$$
\mathrm{R}=\left(\Sigma_{\mathrm{s}}^{\circ} \bar{\ell}\right)\left(\frac{\Sigma_{\mathrm{a}}^{*}}{\Sigma_{\mathrm{a}}^{\circ}}\right)
$$

Now the average absorption cross - section can be expressed by

$$
\Sigma_{a}^{*}=\int_{E=0}^{E_{0}} \Sigma_{a}(E) P(E) \cdot d E
$$

where

$E_{O}$ is the maximum incident energy,

$$
\begin{aligned}
& \Sigma_{a}(E)=\Sigma_{a}^{\circ} \frac{1}{\sqrt{E}}, \text { and } \\
& P(E) d E=\frac{d E}{E} \text { for hydrogen. }
\end{aligned}
$$

Integration of Eq. C-5 shows that the ratio of the average absorption cross-section for scattered neutrons to the average for the initial incident neutrons is:

$$
\frac{\Sigma_{a}^{*}}{\Sigma_{a}^{0}}=2 .
$$


Therefore Eq. C-4 can be rewritten

$$
R=2 \Sigma_{s}^{\circ} \bar{l}
$$

Thus since $\Sigma_{\mathrm{S}}^{0} \approx 2 \mathrm{CM}^{-1}$ for scattering by a hydrogeneous substance and $\bar{l} \approx 1 \mathrm{CM}$ for a reasonably sized sample, the ratio: of the probability of capture reactions by scattered lower energy neutrons to that for the incident higher energy neutrons is:

$$
\mathrm{R}=4
$$

This shows that the hydrogeneous sample target should be very thin to overcome the capture contribution by the scattered neutrons. 
Appendix D

COMPUTER PROGRAM INTEF

INTEF calculates the intrinsic efficiency of a Ge(Li) detection system using a serles of runs with calibrated gamma-ray sources. All data are fitted by the Iinear - least square method. Typical input and output data follow immediately after the program. Input data consists of the energy of the peak, the half-life of the standard source, the initial activity of the source, the yield of the gamma-ray, the cooling time interval, the gamma-attenuation coefficient of the lucite-source-holder, the number of input peaks and the distance and peak area array. Output data give the two coefficients of the least-square fitted linear equation, which will calculate the intrinsic efficiency as a function of the distance from the detector. 
INTRINSTC EFFICIFNCY CALCULATION

REAL. A (21), EFF(21),R(21), EFC(21), EROR(21), CONST(21), COEFF(21), IENERG (21)

READ $(5,3) \mathrm{N}$

3 FORMAT (I 5$)$

DO $200 \quad J=1, N$

READ $(5,1)$ EN, AO, TH,Y,TC, SL, M

1 FORMAT (F10.4,F5.1,4E10.3, I5)

EN - ENERGY OF THE PEAK IN KEV

TH - HALF-LIFE DF THE RADIOACTIVE MATERIAL IN DAYS

AO - INITIAL ACTIVITY IN MICRO-CURIES

$Y$ - YIELD OF GAMMA-RAY

TC - COOLING TIME IN DAYS

SL - GAMMA-ATTENUATION CEFFICIENT OF LUCITE

$N$ - NO. OF DIFFERENT ENERGY GROUPS

$M$ - NO. OF INPUT PEAKS

A - PEAK AREA ARRAY

EFF- EFFICIENCY ARRAY

$R$ - DISTANCE ARRAY IN INCHES

INITIAL ACTIVITY

$A C=A O *(3.7 E+04)$

WRITE $(6,10)$ EN

WRITE $(7,10)$ EN

10 FORMAT $1: 1,14 X$, 'ANALYSIS OF, F10.4, KEV')

WRITE $(6,11) A O$

WRITE $(7,11) A O$

C

11 FORMAT $(10,14 X$, THE INITIAL ACTIVITY (DIS/SEC) ,E10.5)

C DECAY CORRECTION FACTOR CALCULATION

$C D=0.693 / T H$

$C D=C D * T C$

$C D=E X P(-C D)$

WRITE $(6,12) \quad C D$

WRITE $(7,12) \quad C D$ 
C GAMMA-RAY YIELD $Y=Y / 10 \%$

WR ITE $(6,13), Y$

WRITE $(7,13) Y$

C

13 FORMAT $115 X$, THE GAMMA-RAY Y IELD

$\cdot E 10.51$

C LUUCITE ATTENUATION CORRECTION

$C L=1.995$

C CL IS THE THICKNESS OF LUCITE IN C:M

$C L=C L * S L$

$C L=(1-E X P(-C L)) / C L$

WRITE $(6,14) \quad \mathrm{CL}$

WRITE $(7,14) \mathrm{CL}$

14 FOPMAT (I5X, 'THE LUCITE ATtENUATION FACTOR. ', E 10.5)

C

C TOTAL COUNTING TIME

$T D=16$.

C TD IS THE TOTAL COUNTING TIME IN MINUTES

$T D=T O * 6 \%$.

WRITE 16,151 TD

WRITE $(7,15)$ TD

15 FORMAT (15X, TOTAL COUNT ING TIME. (SEC)

WRITE $(6,16)$

WRITE $(7,16)$

c

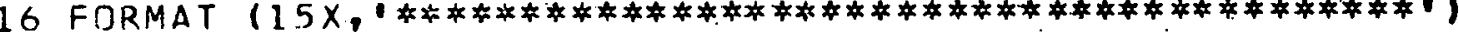

C CONSTANT TERM CALCULATION

$C=A C * C D * Y * C L * T D$

WRITE $(6,17) C$

WRITE $(7,17) \mathrm{C}$

17 FORMAT $(15 X$, 'CONSTANT TERM

$\cdot, E(0.5)$

EFFICIENCY CALCULATION

DO $100 \quad I=1, M$ 
READ $(5,2) \quad R(I), A(I)$

2 FORMAT $(10 \times, F 5.2, E 12.4)$

$E A=A(I) / C$

$E A=S Q R T(E A)$

$E F F(I)=1 / E A$

100 CONTINUE

C

C

C

C

CALCULATION OF COEFFICIENTS OF LINFAR EQUATION. SUBRDUTINE

LILSQ DOES THE LINEAR LEAST SQUARE FIT.

CALL LILSQ (R,EFF, M, CO,CI)

WRITE $(6,18)$

WRITE $(17,18)$

18 FORMAT ('O',6X,'DISTI IN)',6X,'AREA',8X,'1/SQRT(E)',5X,

1'CALC. LSQ',5X,'RMS ERROR')

DO $101 \quad I=1, M$

$E F C(I)=C l * R(I)+C O$

$E R=E F C(I)-E F F(I)$

$E R=E R * E R$

$E R O R(I)=S Q R T(E R) / E F F(I)$

WRITE $(6,19)$ I, R(I), A(I), EFF(I), EFC (I), EROR(I)

WRI TF $(7,19)$ I,R(I), A(I),EFF(I),EFC (I), EROR(I)

19 FORMAT $(1.5,4 X, F 5.2,4 X, E 10.5,4 X, E 10.5,4 X, E 10.5,4 X, E 10.5)$

101 CONTINUE

WRITE $(6,20)$ EN

WR ITE $(7,20)$ EN

20 FORMAT $110 \%, 14 X$, 'THE COEFFICIENTS OF LINEAR EQUATION FOR ' IF10.5, 'KEV')

WRITE $(6,21) \cdot C O$

WRITE $(7,21) \quad C$,

21 FORMAT $\left(15 X,{ }^{\circ} C O=1, E 12.5\right)$

WRITE $(6,22) \quad C 1$

WRITE $(7,22) \mathrm{CI}$

22 FORMAT $\left(15 \mathrm{X},{ }^{\circ} \mathrm{Cl}=1, E 12.5\right)$

ENERG $(J)=E N$ 
$\operatorname{CONST}(J)=\mathrm{CO}$

$\operatorname{COEFF}(J)=C 1$

200 CONTINUE

$\mathrm{C}$

C

DUTPUT ARGUEMENT. THE DISTANCE BETWEEN DETECTOR AND BLANKET IS 82 INCHES: THE DIAMETER OF DETECTDR IS ONF INCH.

$P, D=82$.

WP ITF $(6,23)$

WRITE $(7,23)$

23 FORMAT $111,14 \times$, THF COEFFICIENTS OF THE LINEAR EQUATIONS')

WRITE $(6,24)$

WR ITE $(7,24)$

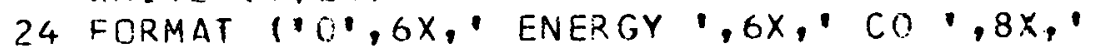

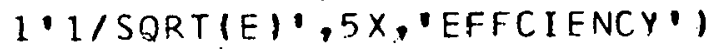

DO $102 \mathrm{~J}=1, \mathrm{~N}$

EFF $(J)=\operatorname{CONST}(J)+R D * \operatorname{COEFF}(J)$

$A(J)=16 * R D * P D /(E F F(J) * E F F(J))$

WRITE $(6,25) \mathrm{J}$, ENERG(J), $\operatorname{CONST}(\mathrm{J}), \operatorname{COEFF}(\mathrm{J}), \operatorname{EFF}(\mathrm{J}), A(\mathrm{~J})$

WRITE $(7,25) \mathrm{J}$, ENERG (J), CONST (J), COEFF (J),EFF(J),A J

25 FORMAT $(I 5,4 X, F 5.0,4 X, E 10.5,4 X, E 10.5,4 X, E 10.5,4 X, E 10.5)$

IC2 COONT INUE

CALL EXIT

END 
SUBROUTINE LILSQ (R, EFF, M, CO,CI)

REAL R(20), EFF(20)

C CO AND CI ARE OUTPUT ARGUEMENTS

$C 11=M$

$\mathrm{C} 12=0$.

$\mathrm{C} 13=\mathrm{C}$.

$\mathrm{C} 22=0$.

C23 $=0$

C

AUGMENTED MATRIX CALCULATION

DO $1 \quad I=1, M$

$\mathrm{C} 12=\mathrm{C} 12+\mathrm{R}(\mathrm{I})$

C.13 $=$ C $13+E F F(I)$

$C 22=C 22+P(I) * R(I)$

$C 23=(23+R(I) * E F F(I)$

1 CONT INUE

$\stackrel{c}{c}$

\section{SOLUTION DF LINEAR EQUATIONS}

DEL TA $=\mathrm{C} 11 * \mathrm{C} 22-\mathrm{C} 12 * \mathrm{C} 12$

$\mathrm{CO}=\mathrm{C} 13 * \mathrm{C}_{2} 2-\mathrm{C} 12 * \mathrm{C}_{2} 3$

$\mathrm{CO}=\mathrm{C} \cap / D E L T A$

$\mathrm{Cl}=\mathrm{C} 11 * \mathrm{C} 23-\mathrm{C} 13 * \mathrm{C}_{12}$

$C 1=C 1 / D E L T A$

RETURN

END 
INPUT DATA

8

\begin{tabular}{|c|c|c|c|c|}
\hline 122.046 & 10.7 & $2.727 E+92$ 8.7.9E+C1 & $8.39: E+32$ & $2.629 F-01$ \\
\hline 1 & 0.00 & $2.5583 E+05$ & & \\
\hline 2 & 1.00 & $1.2781 E+05$ & & \\
\hline 3 & 2.00 & $7.5504 E+04$ & & \\
\hline 4 & 3.00 & $4.8634 F+04$ & & \\
\hline 5 & 4.0 & $3.4280 \mathrm{E}+\mathrm{C}$ & & \\
\hline 6 & 5.00 & $2.5014 E+04$ & & \\
\hline 7 & 6.05 & 1.9.) $85 E+04$ & & \\
\hline 8 & 7.00 & $1.5594 E+04$ & & 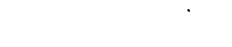 \\
\hline 9 & 8.00 & $1.2346 F+04$ & & \\
\hline 10 & 9.00 & $1.0497 E+94$ & & \\
\hline 11 & 10.00 & $8.6703 E+0.3$ & & \\
\hline 12 & 11.00 & $7.3452 E+03$ & & \\
\hline 136.465 & 10.7 & $2.720 E+021.050 E+01$ & $8.390 E+02$ & $2.501 E-01$ \\
\hline 1 & 0.110 & $3.0386 E+04$ & & \\
\hline 2 & 1.00 & $1.5524 E+04$ & & \\
\hline 3 & 2.00 & $9.3160 \mathrm{E}+\mathrm{U} 3$ & & \\
\hline 4 & 3.00 & $5.4785 E+03$ & & \\
\hline 5 & 4.00 & $3.6650 \mathrm{~F}+03$ & • & \\
\hline 6 & 5.70 & $2.8875 E+13$ & & \\
\hline 7 & 6.00 & $2.4576 E+03$ & & \\
\hline 8 & $7.0 n$ & $1.5808 E+03$ & & \\
\hline 9 & $8.5 n$ & $1.3944 E+1.3$ & & \\
\hline 10 & 9.00 & $1.0046 E+03$ & & \\
\hline 11 & 10.00 & 7. $9760 E+C ?$ & & \\
\hline 12 & 11.10 & $8.971 \mathrm{UE}+02$ & & \\
\hline 511.003 & 9.4 & $9.500 E+021.300 E+02$ & $8.400 E+02$ & $1.452 E-01$ \\
\hline 1 & 0.00 & $2.6354 E+C 5$ & & \\
\hline 2 & 1.00 & $1.3212 \mathrm{E}+05$ & . & \\
\hline 3 & 2.00 & $7.8911 E+04$ & & \\
\hline 4 & 3.00 & $5.2503 E+04$ & & \\
\hline 5 & 4.00 & $3.6728 E+04$ & & \\
\hline
\end{tabular}

12

CO-57

C0-57

CO-57

$\mathrm{CO}-57$

$\mathrm{CO}-57$

$\mathrm{CO}-57$

CO-57

CO-57

$\mathrm{CO}-57$

C. $0-57$

$\mathrm{CO}-57$

CO-57

CO-57

$\mathrm{CO}-57$

CO-57

$\mathrm{CO}-57$

CO-57

$\mathrm{CO}-57$

CO-57

$\mathrm{CO}-57$

CO-57

CO-57

CO-57

CO-57

$\mathrm{CO}-57$

CO-57

NA-22

NA- 22

NA-22

NA- 22

NA- 22

NA-22

PAGE 


\begin{tabular}{|c|c|c|c|c|}
\hline 6 & 5.00 & $2.7353 E+14$ & & \\
\hline 7 & 6.00 & $2.180 C E+C .4$ & & \\
\hline 8 & 7.00 & $1.6735 E+4$ & & \\
\hline 9 & 3.00 & $1.4317 E+04$ & & \\
\hline 10 & 9.00 & $1.1215 E+04$ & & \\
\hline 11 & 10.00 & $9.1933 E+03$ & & \\
\hline 12 & 11.00 & $8.0320 F+03$ & & \\
\hline 661.632 & $12 \cdot 4$ & $1.095 E+04 \quad 3.500 E+01$ & $6.530 E+02$ & $1.287 E-01$ \\
\hline 1 & 0.0 & $2.1378 E+05$ & & \\
\hline 2 & 1.0 & $1.0501 E+35$ & & \\
\hline 3 & 2.00 & $6.3264 E+04$ & & \\
\hline 4 & 4.00 & $2.8375 E+04$ & & \\
\hline 5 & 8.00 & $1.0415 E+04$ & & \\
\hline 6 & 12.00 & $5.6206 E+03$ & . & \\
\hline 7 & 16.00 & $3.5152 E+03$ & & \\
\hline 8 & 20.00 & $2.3264 E+0.3$ & & \\
\hline 9 & 24.00 & $1.6302 \mathrm{E}+03$ & & \\
\hline 834.861 & 10.5 & $3.140 E+021.000 E+C 2$ & $8.400 E+02$ & $1.186 E-01$ \\
\hline 1 & 0.00 & $2.3517 E+C 4$ & & \\
\hline 2 & 1.00 & $1.1173 E+04$ & & \\
\hline 3 & 2.00 & $7.0034 E+03$ & : & \\
\hline 4 & 3.00 & $4.3610 E+C .3$ & & \\
\hline 5 & 4.00 & $3.1072 \mathrm{E}+03$ & & \\
\hline 6 & 5.00 & $2.3204 E+03$ & & \\
\hline 7. & 6.00 & $1.8562 E+03$ & & \\
\hline 8 & 7.00 & $1.4476 E+03$ & & \\
\hline 9 & 3.00 & $1.192 .1 E+03$ & & \\
\hline 10 & 9.00 & $9.8490 \mathrm{E}+02$ & & \\
\hline 11 & 10.20 & $8.0070 E+02$ & n & \\
\hline 12 & 11.00 & $6.7400 E+02$ & & . \\
\hline 1173.231 & 7.5 & $1.920 E+031.000 E+02$ & $6.560 F+02$ & $1.056 E-01$ \\
\hline 1 & 0.20 & $5.7156 E+04$ & & \\
\hline 2 & 1.00 & $2.8621 E+04$ & & \\
\hline 3 & 2.00 & $1.6171 E+04$ & & \\
\hline 4 & 3.00 & $1.1242 E+04$ & & \\
\hline 5 & 4.00 & $8.2430 E+C 3$ & & \\
\hline
\end{tabular}




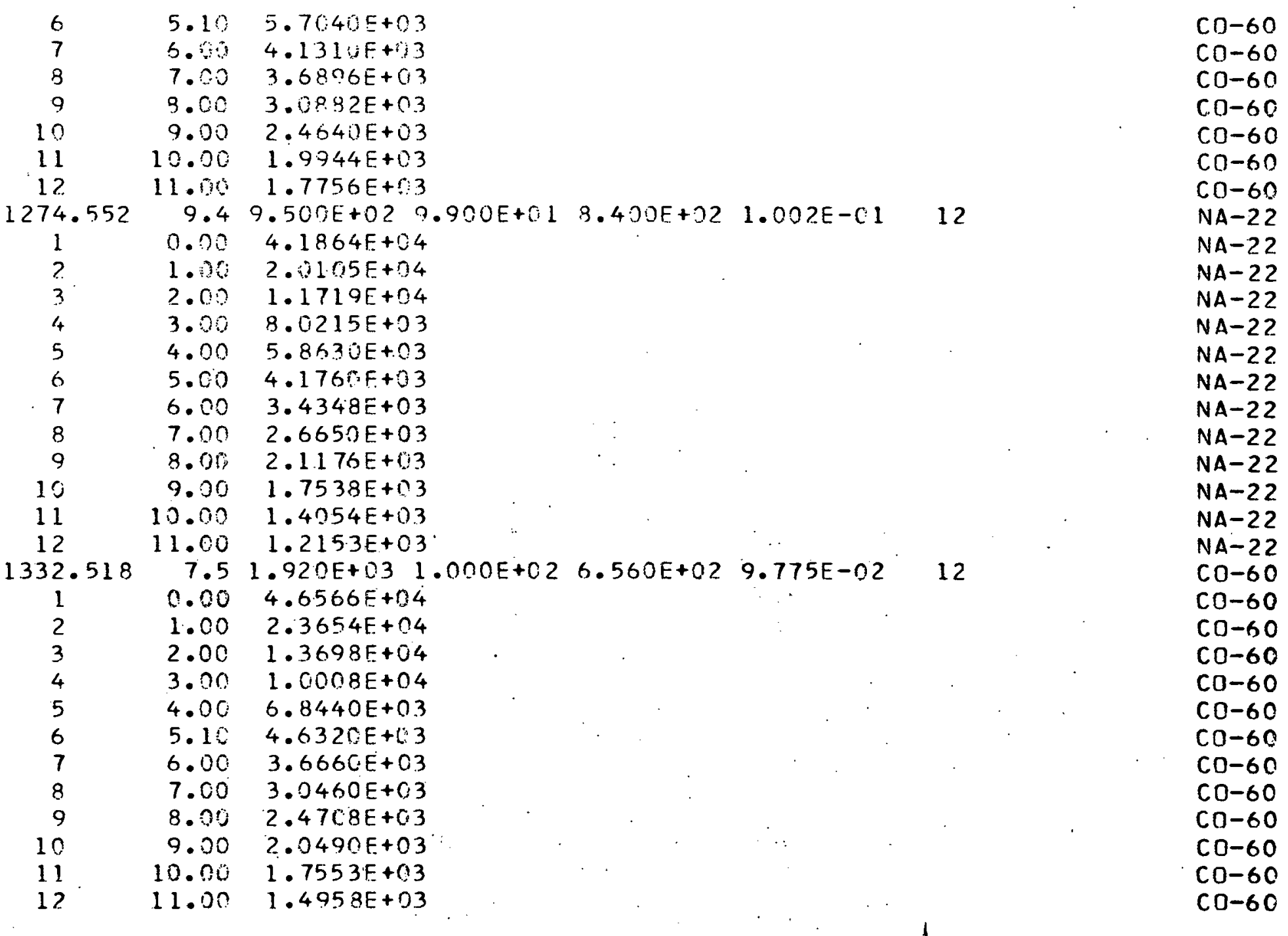


ANALYSIS OF $122.0460 \mathrm{KEV}$

THE INITIAL ACTIVITY (DIS/SEC) .39590F O6 TECAY C.OPRECTICN FACTOR THE GAMMA-RAY YIELD THF LUCITE ATTENUATION FACTOR .78664 OOO TOTAL COUNTING TIME (SFC)

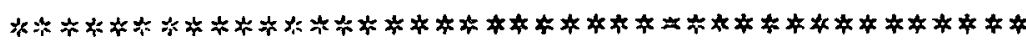
CONSTANT TERP

$.30676 \mathrm{E} 08$

\begin{tabular}{|c|c|c|}
\hline IST(IN) & AREA & \\
\hline 0.0 & $.25583 E$ & 2 \\
\hline 1.00 & $.12731 \mathrm{E}$ & \\
\hline 2.00 & $5504 E$ & \\
\hline $3 . r$ & $.49634 \mathrm{~F}$ & 8 \\
\hline 4.00 & $.34280 E$ & 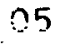 \\
\hline 5.00 & $.25014 F$ & 0 \\
\hline 6.00 & $.19095 \mathrm{~F}$ & \\
\hline 7.00 & $.15594 \mathrm{E}$ & \\
\hline .00 & $.12346 \mathrm{E}$ & \\
\hline 00 & $.10497 E$ & \\
\hline 00 & .867 & \\
\hline & & \\
\hline
\end{tabular}

CALC. LSQ $.15465 E \quad 02$ $.20345 E \quad 02$ $.25225 E \quad 02$ $.30105 E 02$ $34985 \mathrm{E} \quad 02$ $.39866 E$ O2 $.44746 \mathrm{~F} \mathrm{O2}$ $.49626 \mathrm{E} \mathrm{O2}$ $.54505 E 02$ $.59386 E$ O2 $.64266 \mathrm{~F} \mathrm{O2}$ .10585 E 02
RMS ERROR $.33348 E-01$ $.17583 \mathrm{E}-\mathrm{C} 2$ $.93597 \mathrm{E}-02$ $.43945 E-02$ $.63801 \mathrm{E}-\mathrm{C} 2$ $.97471 E-03$

$.56465 E-C 2$ $.88491 \mathrm{~F}-02$

$.44420 E-02$ . $82584 E-02$

$.16182 \mathrm{E}-02$

$.55586 E-02$

THE COEFFICIENTS DF LINEAR EQUATION

$C O=0.10585 \mathrm{E} 02$

$C_{1}=0.48801 \mathrm{E} 01$ 
ANALYSIS DF $136.4650 \mathrm{KEV}$

THE (NITIAL ACTIVITY (DIS/SEC) .39590E O6 THE DECAY CORRECTION FACTOR .11794E DO THE GAMMA-RAY YIELD .1050OE OC THE LUCITE ATTENUATIDN FACTUR .79550E DO TOTAL COUNTING TIME (SEC) .95000E 03

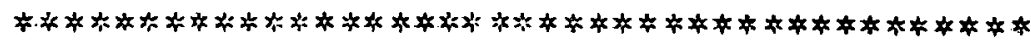
CONSTANT TERM

$.37440 \mathrm{~F} 07$

$\begin{array}{cc} & \text { OISTIINI } \\ 1 & 0.0 \\ 2 & 1.00 \\ 3 & 2.00 \\ 4 & 3.00 \\ 5 & 4.00 \\ 6 & 5.00 \\ 7 & 6.00 \\ 8 & 7.00 \\ 9 & 8.50 \\ 10 & 9.00 \\ 11 & 10.00 \\ 12 & 11.00\end{array}$

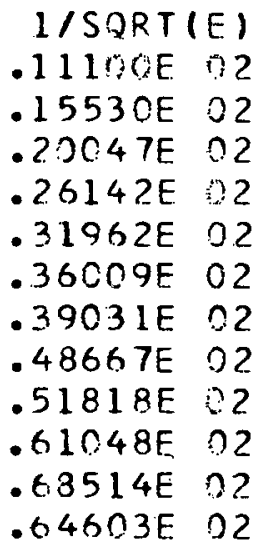

CALC. LSQ $.10041 E \quad 02$

$.15404 E 02$

$.20768 \mathrm{E} 02$

$.26131 E 02$

$.31494 \mathrm{E} \quad 02$

.36857E O2

$.42221 \mathrm{E} \mathrm{O2}$

.47584 E C2

$.52947 E$ Q2

$.58311 \mathrm{E} 02$

$.63674 \mathrm{E} \quad 02$

.69037 E 02
RMS ERROR

. 95426E-01

. 80869E-02

$.35932 \mathrm{E}-01$

$.42609 E-03$

$.14634 \mathrm{E}-01$

$.23568 \mathrm{E}-01$

- $81712 \mathrm{E}-01$

. $22245 \mathrm{E}-01$

- $21805 \mathrm{E}-01$

$.44844 \mathrm{E}-01$

$.70639 E-01$

$.68646 \mathrm{E}-01$

THE COEFFICIENTS OF LINEAR EQUJATION

$C O=0.10041 E 02$

$C 1=0.53633 \mathrm{E} \mathrm{Cl}$ 
ANALYSIS OF 511.0029KFV -

THE INITIAL ACTIVITY (OIS/SEC) .3478CE O6 THE DECAY CORRECTICIN FACTIR . $54185 E$ CO THE GAHMA-RAY YIELD $\quad .18000$ OI THE LUCITE ATTENUATION FACTOR .87361E OO TOTAL COUNTING TIME (SEC) .960COE 0.3

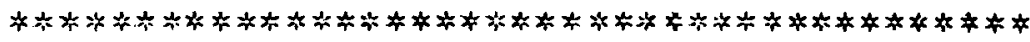
CONSTANT TFRM

.28450 O O9

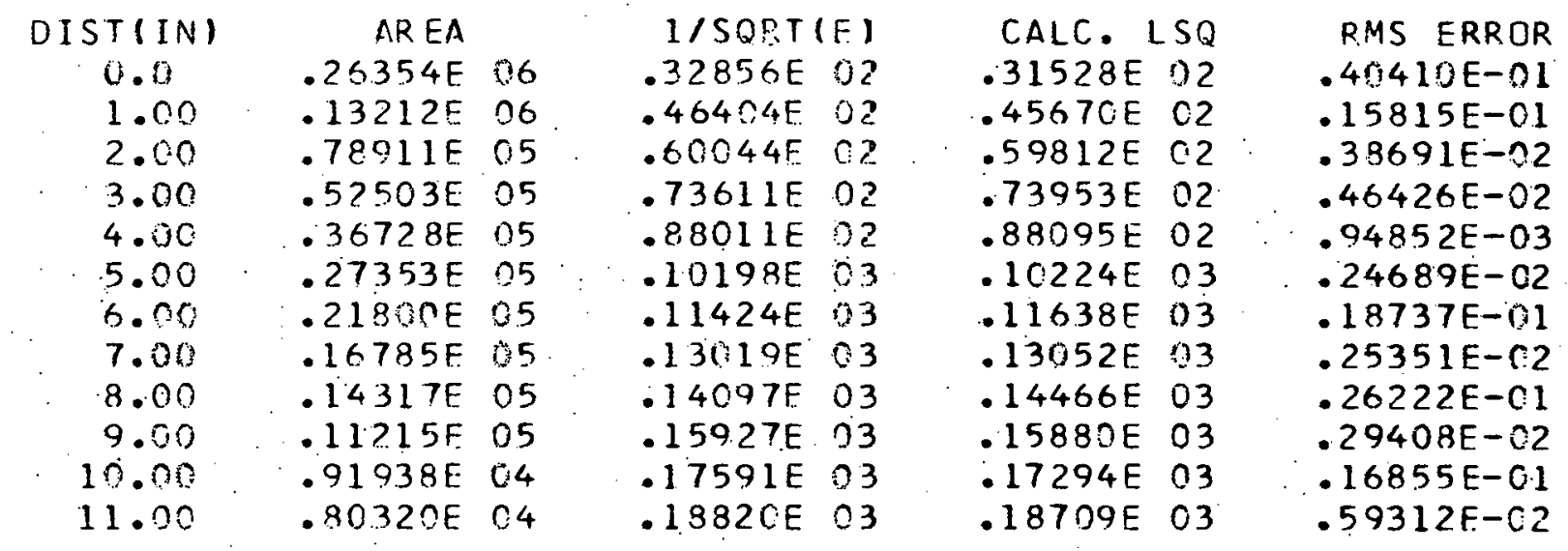

THE COEFFICIENTS CF LINEAR EQUATION

$\mathrm{CO}=0.31528 \mathrm{E} \mathrm{O2}$

$C 1=0.14142 \mathrm{~F} 02$ 


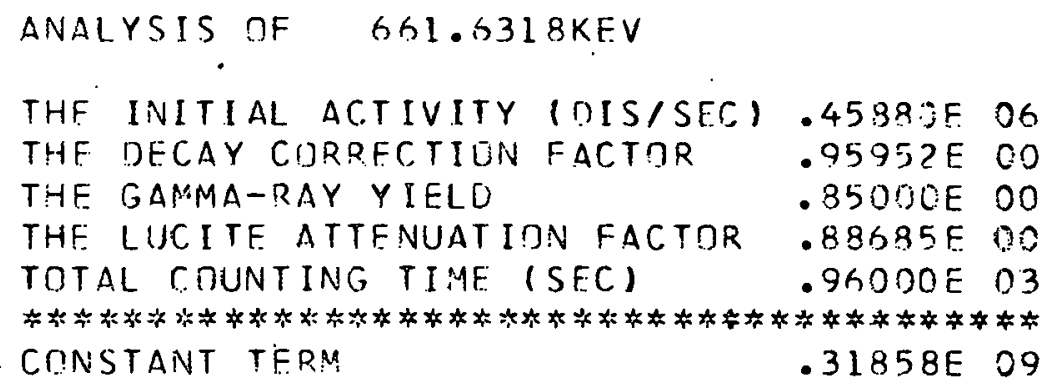

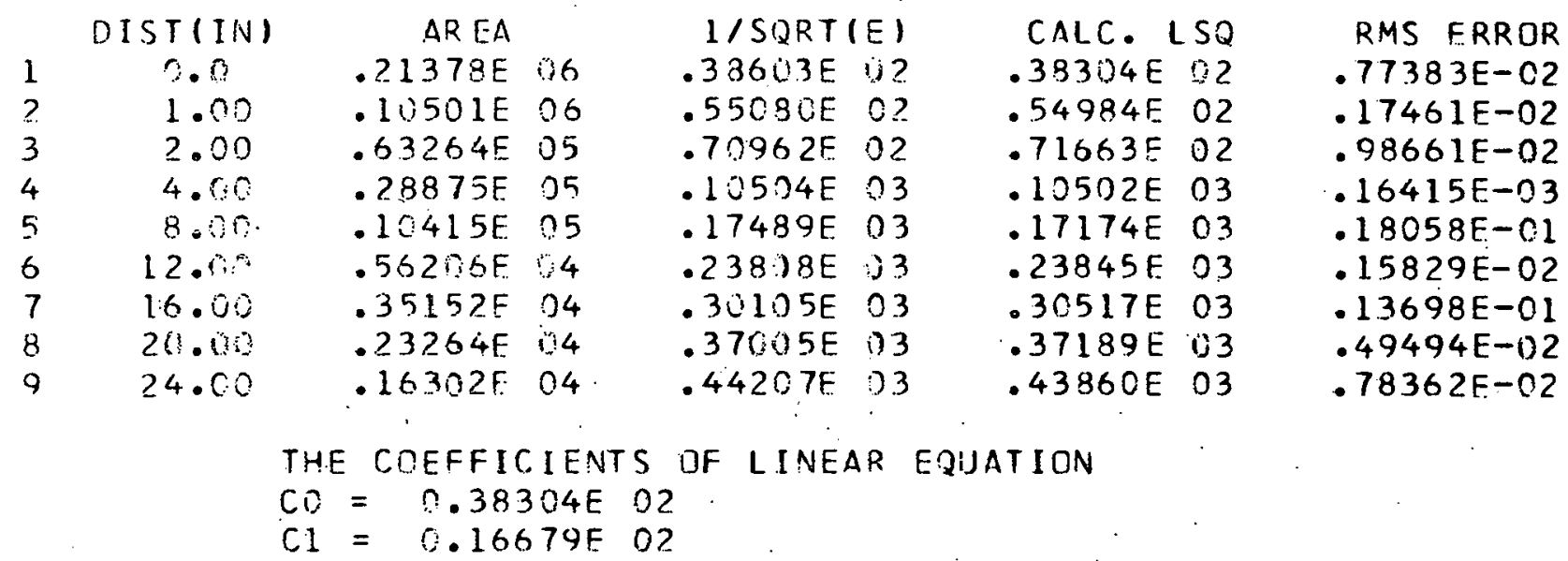


ANALYSIS OF 8.34.860.3KEV

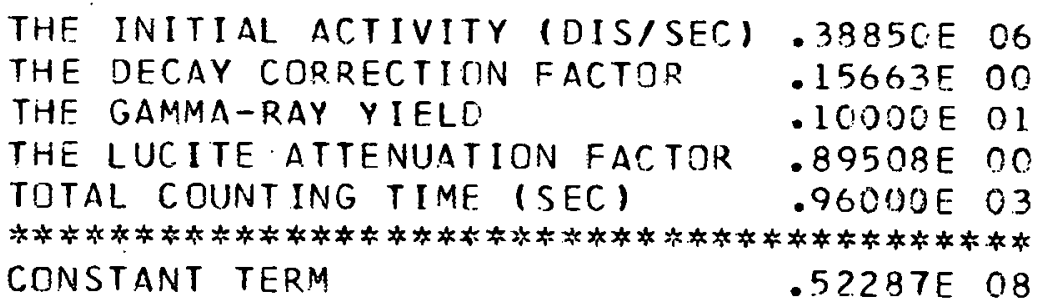

RMS ERROR $.22122 E-O 1$ $.22585 \mathrm{E}-\mathrm{Cl}$ $.14030 E-01$ $.10276 E-01$ $.45877 E-02$ $.15410 E-C 2$

$.16678 \mathrm{E}-01$

$.70350 E-.02$

$.12952 \mathrm{E}-01$

$.10797 \mathrm{E}-\mathrm{Cl}$

$.73960 \mathrm{E}-02$

$.14794 \mathrm{E}-01$

THE COEFFICIENTS OF LINEAR EQUATION

CO $=0.46109 E \quad 2$

$C_{1}=0.20754 E \quad 2$ 
DUTPUT DATA

ANALYSIS OF $1173.2310 \mathrm{KEV}$

THE INITIAL ACTIVITY (DIS/SEC)

THE DECAY CORRECTION FACTDR

THE GAMIMA-RAY YIELD

THE LUCITE ATTENUATION FACTDR

TOTAL COUNTING TIMF (SEC)

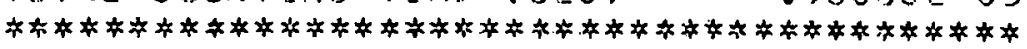

CONSTANT TERM

$$
.19044 E \quad 09
$$

$\begin{array}{cc} & \text { DIST(IN) } \\ 1 & 0.0 \\ 2 & 1.00 \\ 3 & 2.00 \\ 4 & 3.00 \\ 5 & 4.00 \\ 6 & 5.10 \\ 7 & 6.09 \\ 8 & 7.00 \\ 9 & 8.00 \\ 10 & 9.00 \\ 11 & 10.00 \\ 12 & 11.00\end{array}$

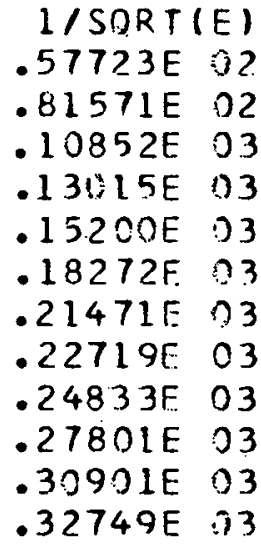

CALC. LSQ .57333 E 02 .81959E C2 .10662 E 03 $.13127 \mathrm{E} 03$ $.15593 \mathrm{E} \mathrm{O3}$ $.18305 \mathrm{~F} 03$ $.20524 E \cap 3$ $.22990 E 03$ $.25455 E \quad 03$ .27921 .03 .30386E 03 .32852 E 03
RMS ERROR $.72640 E-02$ $.47660 \mathrm{E}-02$

$.17545 E-01$

$.85948 E-02$

$.25865 E-01$

$.18031 \mathrm{E}-02$

$.44097 \mathrm{E}-01$

$.11918 \mathrm{E}-01$

$.25071 \mathrm{E}-01$

$.43224 E-02$

$.16645 E-01$

$.31347 \mathrm{E}-02$

THE COEFFICIENTS OF LINEAR EQUATION

$\mathrm{CO}=0.57303 \mathrm{E} \mathrm{O2}$

$C 1=0 . ? 4656 E$ O? 
ANALYSIS OF $1274.5513 \mathrm{KEV}$

THE INITIAL ACTIVITY (DIS/SEC) • 34780E 06 THE DECAY CORRECTION FACTOR .

THE GAMMA-RAY YIELD

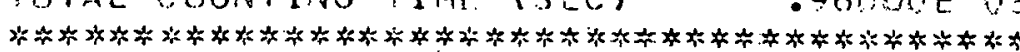

CONSTANT TERM

$.16305 E 09$

$\begin{array}{cc} & \text { DISTIIN) } \\ 1 & 0.0 \\ 2 & 1.00 \\ 3 & 2.00 \\ 4 & 3.00 \\ 5 & 4.00 \\ 6 & 5.00 \\ 7 & 6.00 \\ 8 & 7.00 \\ 9 & 8.00 \\ 10 & 9.00 \\ 11 & 10.00 \\ 12 & 11.00\end{array}$

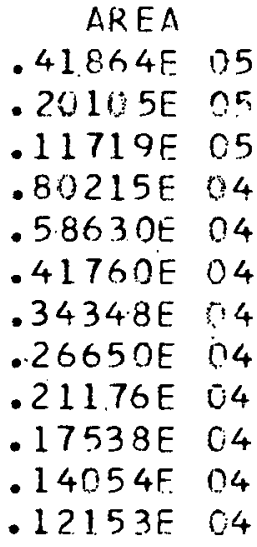

$-1 / S Q R T$ (E) $.62408 \mathrm{~F} 02$ $.90056 \mathrm{E} .22$

$.11796 \mathrm{G} 03$

$.14257 \mathrm{E} \ 3$

$.16676 E 03$

.19760 E 03

$.21788 E \quad 03$

$.24735 E \quad 03$

.27 .749 D D3

$.30491 E$ J3

$.34062 E \quad 03$

$.366 .29 \mathrm{E} \quad 03$
CALC. LSQ $.60164 E 02$ .87587 E 02

$.11501 E 03$

$.14243 E \quad 3$

$\therefore 16986 \mathrm{E} \mathrm{O3}$

.19728 E O3

.2247 CE 03 $.25213 \mathrm{~F} 03$ $.27955 \mathrm{~F} 03$ $.30697 \mathrm{E} \mathrm{O3}$ $.33439 \mathrm{E} \mathrm{O} 3$ $.36182 \mathrm{E} 03$
RMS ERRDR .35969E-01 $.27417 E-01$ $.24974 \mathrm{E}-\mathrm{O}$ . $98024 E-03$

$.18537 \mathrm{E}-01$

. $16168 \mathrm{E}-02$

$.31321 \mathrm{E}-01$

. $19297 \mathrm{E}-01$

$.74293 E-02$

$.67546 \mathrm{E}-02$

. $18265 \mathrm{E}-01$

$.12205 \mathrm{E}-\mathrm{Cl}$

THE COFFFICIFNTS OF LINNEAR EQUATION

$\mathrm{CO}=0.60164 \mathrm{E} \quad 02$

$C 1=0.27423 E 02$ 
ANALYSIS OF $1332.5178 \mathrm{KEV}$

THE INITIAL ACTIVITY IDIS/SFCI .27750E 06 THE DECAY CORRECTION FACTOR

THE GAMMA-RAY YIELD

HOCOE 01

THE LUCITE ATTENUATIION FACTOR. $.91241 \mathrm{E} 00$

TOTAL COUNTING TIME (SFC)

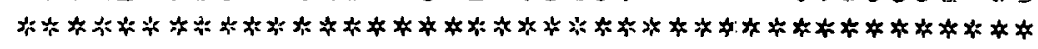

CONSTANT TFRM

$.19182 \mathrm{E} .09$

$\begin{array}{cc} & \text { DISTIIN) } \\ 1 & 0.0 \\ 2 & 1.00 \\ 3 & 2.00 \\ 4 & 3.00 \\ 5 & 4.00 \\ 6 & 5.10 \\ 7 & 5.00 \\ 8 & 7.00 \\ 9 & 8.00 \\ 10 & 9.00 \\ 11 & 10.00 \\ 12 & 11.00\end{array}$

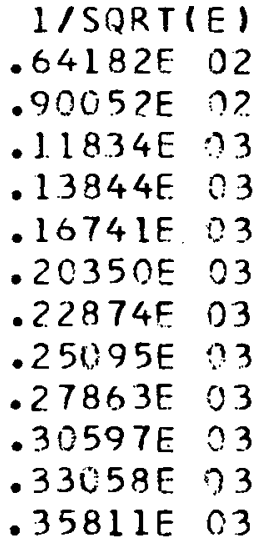

CALC. LSQ

. $63106 E 02$ $89999 \mathrm{E} \quad 2$

$.11689 \mathrm{E} 03$

$14378 E \quad 03$

.17068 E 03

$.20026 E 03$

$.22446 E 03$

$.25136 E 03$

$.27825 E \quad 03$

$.30514 E 03$

$.33204 E 03$

$.35893 E 03$
RMS ERROR

.16766E-01

$.59407 E-03$

$.12208 \mathrm{E}-01$

$.38579 E-01$

. 19494E-01

- $15918 \mathrm{E}-01$

. $18715 \mathrm{E}-01$

- $16314 E-02$

$.13660 E-02$

$.26978 E-02$

$.44149 \mathrm{E}-02$

$.22989 E-02$

THE CDEFFICIENTS DF LINEAR EQUATION

$C O=C .63106 \mathrm{E} \mathrm{O2}$

$C 1=0.26893 \mathrm{E} 02$ 
THE COFFFICIENTS OF THE LINEAR EQUATIONS

\begin{tabular}{|c|c|c|c|c|c|c|c|}
\hline ENERGY & \multicolumn{2}{|l|}{$\mathrm{CO}$} & \multicolumn{2}{|l|}{$C 1$} & \multicolumn{2}{|c|}{$1 / \operatorname{SQR} T(E)$} & EFFCI ENCY \\
\hline 122 & $.10585 E$ & 02 & $.48801 \mathrm{E}$ & $D 1$ & $.41075 \mathrm{~F}$ & 33 & $.63766 \mathrm{E} \mathrm{OO}$ \\
\hline 136. & $10041 \mathrm{~F}$ & 02 & $.53633 \mathrm{E}$ & 81 & $.44983 E$ & 03 & $.53168 E 00$ \\
\hline 511 . & $1528 \mathrm{E}$ & 02 & $.14142 \mathrm{E}$ & 02 & $.11911 \mathrm{E}$ & 04 & $.75826 \mathrm{E}-01$ \\
\hline 662. & $.38304 \mathrm{E}$ & 02 & $.16679 \mathrm{E}$ & 22 & $.14060 \mathrm{E}$ & 04 & $.54423 E-01$ \\
\hline 835. & $.46109 E$ & 02 & $.20754 E$ & 02 & $.17480 E$ & 04 & $.35212 \mathrm{E}-01$ \\
\hline 1173. & $.57303 E$ & 02 & $.24656 E$ & $0 ?$ & .207915 & 04 & $.24888 E-01$ \\
\hline 1275 . & $.60164 \mathrm{~F}$ & 02 & $.27423 E$ & 92 & $.23089 \mathrm{E}$ & 04. & $.20182 E-01$ \\
\hline 133 & $.63106 E$ & 02 & $.26893 \mathrm{E}$ & 02 & $.22683 E$ & 04 & $309 \mathrm{E}$ \\
\hline
\end{tabular}




\section{Appendix $\mathrm{E}$ \\ CALCULATION OF GAMMA-RAY SELF-ABSORPTION CORRECTION FACTORS FOR BLANKET NO. 2}

\section{E-1 Theory}

The gamma-ray self-absorption correction factor is defined as the ratio of the total number of gamma-rays of interest with energy $E \gamma$ appearing at the outside surface of the blanket per second to the total number of gamma-rays of interest with the same energy Er produced throughout the blanket per second. Hence this factor is mainly dependent on the structure of the blanket and the origin of the gamma-rays of interest, for example, suppose that the gamma-ray of interest is one of the decay gamma-rays from $\mathrm{Np}-239$, then the gammaray originates from the fuel rod and goes through Region A of the blanket, which is shown in Fig. E-I. However if the gamma is one of the decay gamma-rays from $\mathrm{Na}^{24}$, the gamma-ray originates from $\mathrm{Na}_{2} \mathrm{CrO}_{4}$ and goes through Region A or Region B of the blanket. In other words, the gamma-ray self-absorption in Blanket No.? differs according to the energy of gamma-rays and the place of origin of the gamma-rays.

Figure E-l shows the detail of the blanket structure seen by the detector, looking down the 2-inch diameter 


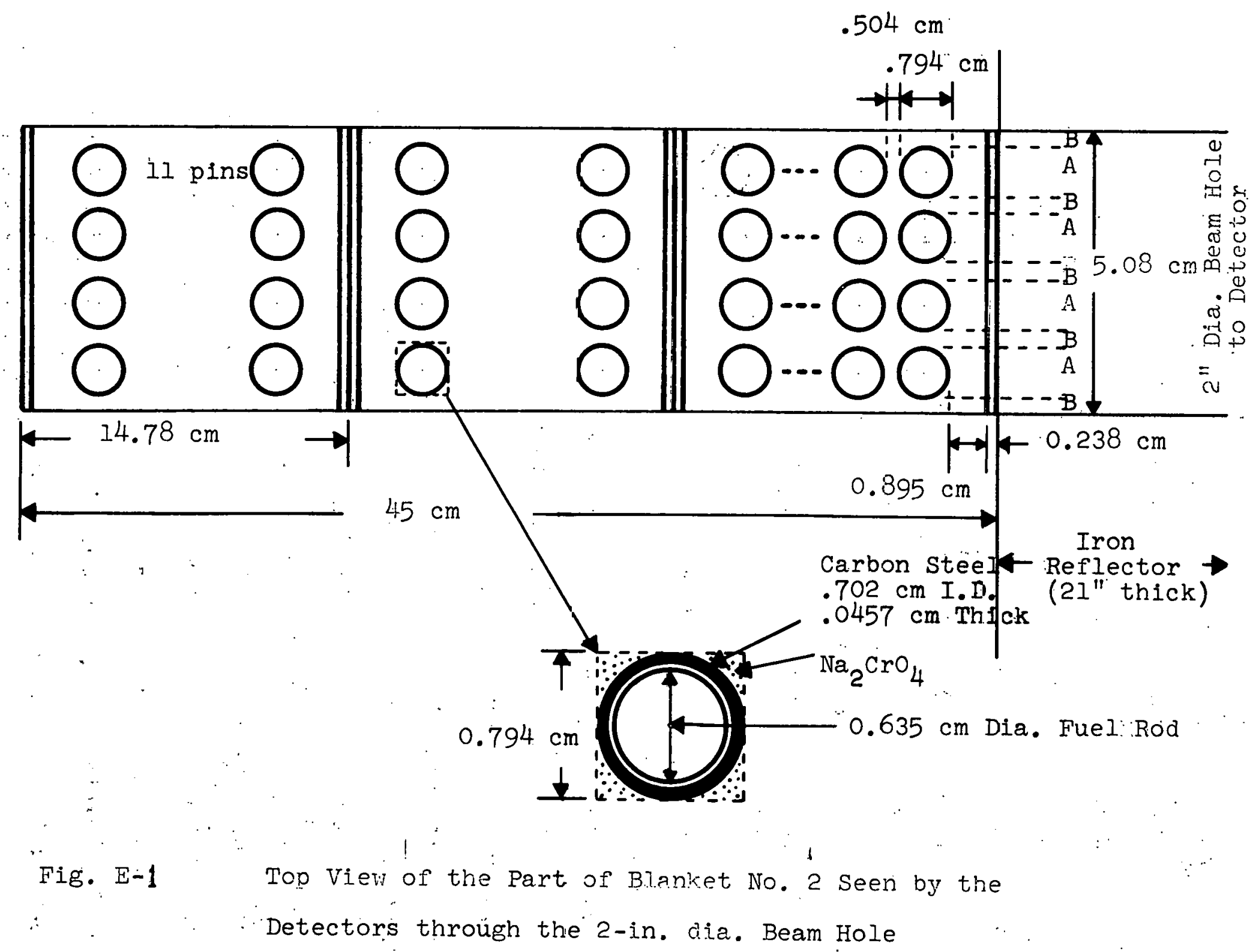


beam hole penetrating the reflector. The structure is divided into two regions, $A$ and $B$, where Region $A$ contains the fuel pins and $\mathrm{Na}_{2} \mathrm{CrO}_{4}$, and Region $\mathrm{B}$ contains $\mathrm{Na}_{2} \mathrm{CrO}_{4}$ only.

Suppose the gamma-ray of interest with energy Er originates from $\mathrm{Na}_{2} \mathrm{CrO}_{4}$ in the blanket; the selfabsorption correction factor is given by

$$
S \gamma=\int_{\Delta V} \operatorname{dv} \psi(\underline{r}) n(\underline{r}) S \gamma(\underline{r})
$$

or

$$
S \gamma=\int_{V_{A}} d V \psi(\underline{r}) n(\underline{r}) S \gamma(\underline{r})+\int_{V_{B}} d V \psi(\underline{r}) n(\underline{r}) S \gamma(\underline{r})
$$

where $\mathrm{V}_{\mathrm{A}}$ and $\mathrm{V}_{\mathrm{B}}$ are volume fractions of $\mathrm{Na}_{2} \mathrm{CrO}_{4}$ in Region $A$ and Region $B$, respectively.

Region $B$ is a completely homogeneous medium of $\mathrm{Na}_{2} \mathrm{CrO}_{4}$, and so the self-absorption correction factor for Region $B$ only is simply:

$$
\begin{aligned}
& (S \gamma)_{B}=\frac{v_{B}}{3} \cdot\left(\int_{0}^{I} \psi(x) S \gamma(x) d x\right) \cdot e^{-\mu} \mu_{\gamma}^{F e} \cdot d \\
& +\frac{V_{B}}{3} \cdot\left(\int_{0}^{l} \psi(x) S \gamma(x) d x\right) \cdot e^{-3 \mu_{\gamma}^{F e}}: d \cdot e^{-\mu_{\gamma}^{N} \cdot 1} \\
& +\frac{V_{A}}{3} \cdot\left(\int_{0}^{I} \psi(x) s \gamma(x) d x\right) \cdot e^{-5 \mu_{\gamma}^{F e} \cdot d} \cdot e^{-2 \mu \mu_{\gamma}^{N} \cdot I}
\end{aligned}
$$

where $\mu_{\gamma}^{\mathrm{Fe}}$ and $\mu_{\gamma}^{\mathrm{N}}$ are the linear attenuation coefficients of gamma-rays with energy $\mathrm{E} \gamma$ for iron and $\mathrm{Na}_{2} \mathrm{CrO}_{4}$, respectively, 
and $d, 1$ - are the thicknesses of the steel plate of the subassembly wall and the subassembly box less the thickness of its two steel walls. The first term of Eq. E-2 is the contribution from the first subassembly; the second term from the second subassembly; and the third term from the third sub-assembly. The neutron flux shape, $\psi(x)$, is adequately approximated by an exponential function (see Appendix A):

$$
\Psi(x)=a e^{-\Gamma x}
$$

where

$$
\begin{aligned}
& a=0.662 \text { and } \\
& \Gamma=0.0621 \mathrm{~cm}^{-1} .
\end{aligned}
$$

And the self-absorption function, $s \gamma(x)$, is:

$$
S \gamma(x)=e^{-\mu_{\gamma}^{N}\left(x_{B}-x\right)}
$$

where $\mathrm{X}_{B}$ is the thickness of the blanket, as usual.

Region. A is somewhat more complicated. As one can see in Fig. E-2, Region A is divided into three parts, the steel plate (d), the end piece of $\mathrm{Na}_{2} \mathrm{CrO}_{4}$ (In) at both ends of the subassembly, and successive eleven unit cells of fuel pin plus $\mathrm{Na}_{2} \mathrm{CrO}_{4}\left(2 \mathrm{R}_{\mathrm{N}}\right)$. The geometry of the plate and the $\mathrm{Na}_{2} \mathrm{CrO}_{4}$ at both ends of the subassembly depends only on the axial $x$ direction. However, that of the unit cell, which consists of a fuel 


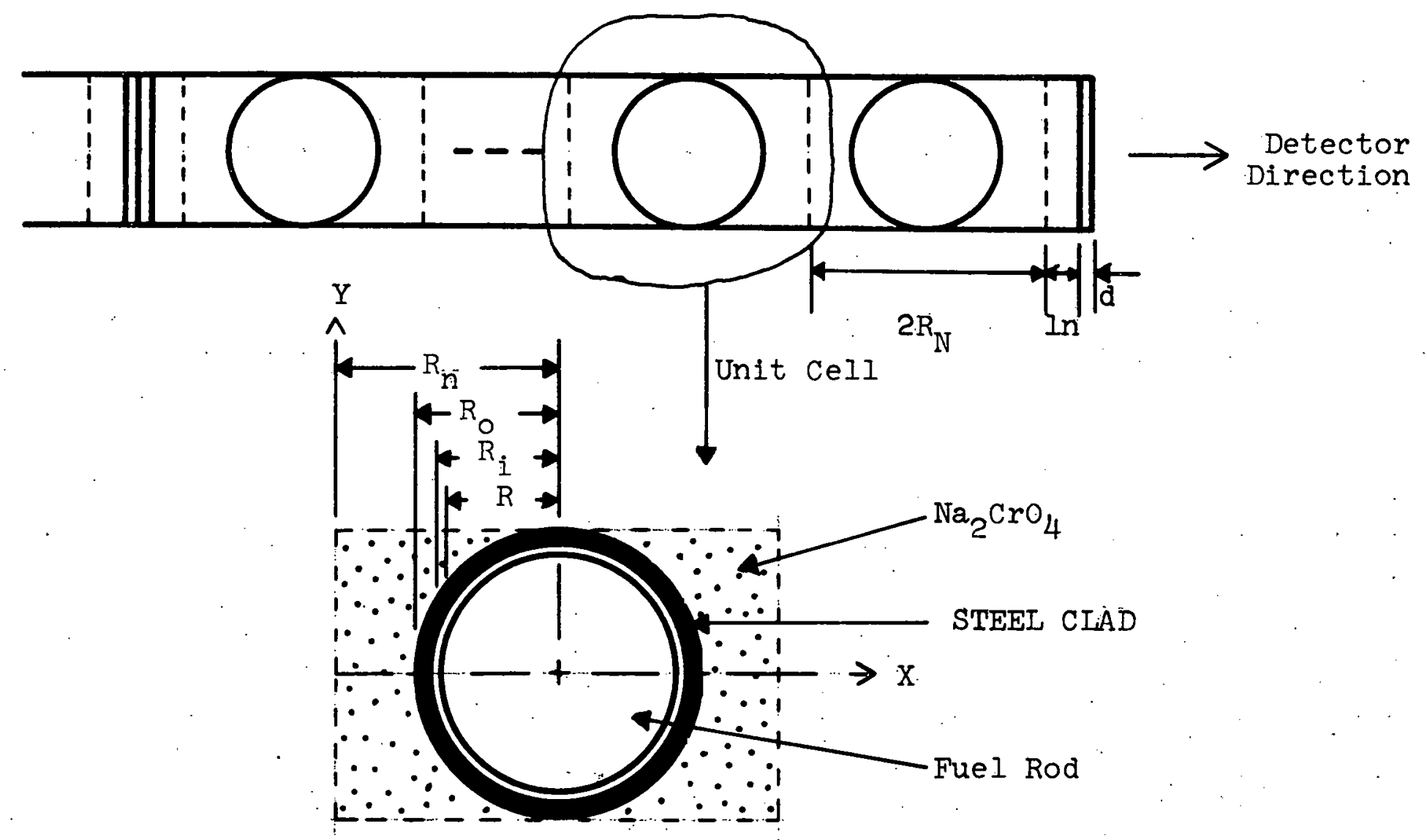

Fig. E-2

Top View of Region A of Blanket No. 2 Seen

by the Detector, and a Unit Cell 
pin and $\mathrm{Na}_{2} \mathrm{CrO}_{4}$, depends upon two directions, $x$ and $y$. In the unit cell of Fig. E-2,

the thickness of $\mathrm{Na}_{2} \mathrm{CrO}_{4}$ at $\mathrm{y}: 2\left(\mathrm{R}_{\mathrm{N}}-\sqrt{\mathrm{R}_{\mathrm{O}}^{2}-\mathrm{y}^{2}}\right)=2 \mathrm{X}_{\mathrm{N}}(\mathrm{y})$

the thickness of Fe clad at $y: 2\left(\sqrt{R_{0}^{2}-y^{2}}-\sqrt{R_{i}^{2}-y^{2}}\right)$

$$
=2 \mathrm{X}_{\mathrm{Fe}}(\mathrm{y})
$$

the thickness of Fuel at $y: \quad 2 \sqrt{R^{2}-y^{2}}=2 x_{U}(y)$.

Hence the self-absorption correction factor of the unit cell for the gamma-rays which originate from $\mathrm{Na}_{2} \mathrm{CrO}_{4}$. at the position $\mathrm{y}$ is:

$$
\begin{aligned}
s \gamma(y) & =x_{N}(y) \int_{0}^{X_{N}(y)} \psi(x) s \gamma(x, y) d x \\
& +x_{N}(y)\left(\int_{0}^{\dddot{X}_{N}(y)} \psi(x) s \gamma(x, y) d x\right) \\
& : e^{-\left(\mu_{\gamma}^{F e} \cdot 2 x_{F e}(y)+\mu_{\gamma} \cdot 2 x_{U}(y)\right.} \\
& \left.+\mu_{\gamma}^{N} \cdot x_{N}(y)\right)
\end{aligned}
$$

where

$$
S \gamma(x, y)=e^{-\mu_{\gamma}^{N}}\left(x_{N}(y)-x\right) .
$$

The average self-absorption correction factor for the unit cell, $\mathrm{S} \gamma^{\mathrm{U}}$, can be obtained by integration of Eq. $\mathrm{E}-5$ 
with respect to $\mathrm{y}$. This resultant double integration can be performed numerically.

The attenuation factor of the unit cell for gammarays of energy Er at the position $\mathrm{y}$ is:

$$
\begin{aligned}
A_{\gamma}^{U}(y) & =e^{-2\left(\mu_{\gamma}^{F e}\right.} x_{F e}(y)+\mu_{\gamma}^{U} \cdot X_{U}(y)+\mu_{\gamma}^{N} \quad(E-6) \\
& \left.\cdot X_{N}(y)\right)
\end{aligned}
$$

Similarly the average attenuation factor for the unit cell, $\mathrm{A}_{\gamma}^{\mathrm{U}}$ is obtained by integration of Eq. E-6.

Hence the total self-absorption correction factor for Region $A$ is given by:

$$
\begin{aligned}
(S \gamma)_{A} & =S_{\gamma}^{l n} \cdot A_{\gamma}^{d} \cdot V_{l_{1}} \\
& +S_{\gamma}^{U} \cdot A_{\gamma}^{l n} \cdot A_{\gamma}^{d} \cdot V_{I_{2}} \\
& +S_{\gamma}^{U} \cdot A_{\gamma}^{U} \cdot A_{\gamma}^{2 l n} \cdot A_{\gamma}^{d} \cdot V_{I_{2}}
\end{aligned}
$$

where

$$
+----
$$

$$
\begin{aligned}
& S_{\gamma}^{\ln }=\int_{0}^{\ln } 4(x) e^{-\mu_{N}^{\gamma}(\ln -x) d x} \\
& A_{\gamma}^{d}=e^{-\mu_{\gamma}^{F e}} \cdot d \\
& A_{\gamma}^{l n}=e^{-\mu_{\gamma}^{F e}} \cdot \ln , \quad \text { and }
\end{aligned}
$$


$\mathrm{V}_{1}, \mathrm{~V}_{1_{2}}$, are the volume fractions of $\mathrm{Na}_{2} \mathrm{CrO}_{4}$ in the first remaining $\mathrm{Na}_{2} \mathrm{CrO}_{4}$ part and the unit cell, respectively. Hence the total self-absorption correction factor for gamma-rays having energy $E \gamma$ which originate from $\mathrm{Na}_{2} \mathrm{CrO}_{4}$ is:

$$
S \gamma=(S \gamma)_{A}+(S \gamma)_{B}
$$

This calculation is done by a computer program SELFN. The self-absorption correction factor for gamma = rays which originate from the $U$-fuel rods should be treated differently since Region $A$ is our only concern in this case. As for the decay gamma-rays of Np-239, which is formed in U-fuel rods, the self-abosorption correction factor of the unit cell (see Fig. E-2) for these gamma-rays of energy $E \gamma$ is:. .

$$
s_{\gamma}^{U}=2 \int_{0}^{R} s_{\gamma}^{U}(y) d y
$$

where

$$
\begin{aligned}
S_{\gamma}^{U}(y)= & 2 x_{U}(y)\left(\int_{0}^{X_{U}(y)} \psi(x) \cdot S_{\gamma}(x, y) d x\right) \cdot \\
& \left.: e^{-\left(\mu_{\gamma}^{F e}\right.} \cdot x_{F e}(y)+\mu_{\gamma}^{N} \cdot x_{N}(y)\right)
\end{aligned}
$$

and

$$
S_{\gamma}(x, y)=e^{-\mu_{\gamma}^{U}}\left(x_{N}(y)-x\right)
$$


Hence the total self-absorption correction factor for gamma-rays having energy $\mathrm{E} \gamma$ which originate from U-fuel rods is:

$$
\begin{aligned}
S^{\gamma} & =S_{\gamma}^{U} \cdot A_{\gamma}^{I n} \cdot A_{\gamma}^{d} \cdot V \\
& +S_{\gamma}^{U} \cdot A_{\gamma}^{U} \cdot A_{\gamma}^{l n} \cdot A_{\gamma}^{d} \cdot V \\
& +S_{\gamma}^{U} \cdot\left(A_{\gamma}^{U}\right)^{2} \cdot A_{\gamma}^{I n} \cdot A_{\gamma}^{d} \cdot V \\
& +\cdots
\end{aligned}
$$

where $V=1 / 132$; volume fraction of a single $U$-fuel rod. A computer program SELFU calculates the selfabsorption factor for these gamma-rays.

E-2 Experimental Correction for Fuel Heterogenity

A mock-up experiment was performed to measured the actual attenuation factor of a single fuel rod. The calculated attenuation factor for the unit cell is inexact due to contributions by the statistical misalignment of fuel rod rows and streaming in the gap between fuel and clad. Hence the experimental determination was considered essential.

The test box was built from $6 " \times 6 " \times 21-1 / 2 "$ by 1/16" thick steel plate. This box holds 44 fixed fuel 
rods, 10 movable fuel rods and one irradiated: rod (see Fig. E-3). This mocks up the five center rows of a real subassembly of Blanket No. 2. Sodium chromate fills the space between the rods. The irradiated" rod contains a 1 inch long fuel rod at the center of an aluminum rod. This rod was irradiated in the $2 \mathrm{CHI}$ irradiation facility of the MITR to produce a low energy gamma-ray source of $\mathrm{Np}-239$. A series of measurements were carried out, changing the position of the irradiated rod along the center row of the test box. This was carried out in the isolated accelerator vault with a 35-c.c Ge(Li) crystal. Figure E-4 shows the gamma-ray transmission factors of a unit cell according to the gamma-ray energy. Here the transmission factor of a unit cell is defined by the ratio of transmitted gamma-rays through the unit cell to total incoming gamma-rays to the unit cell. These experimental data are fitted in the linear least-square sense. E-3 Computer Programs SELFN and SELFU

Two computer programs, SELFN and SELFU, are listed together with typical input and output data. This program mainly performs numerical double integrations. Between two mesh points Simpson's rule is applied to perform a simple integration. The calculated values 

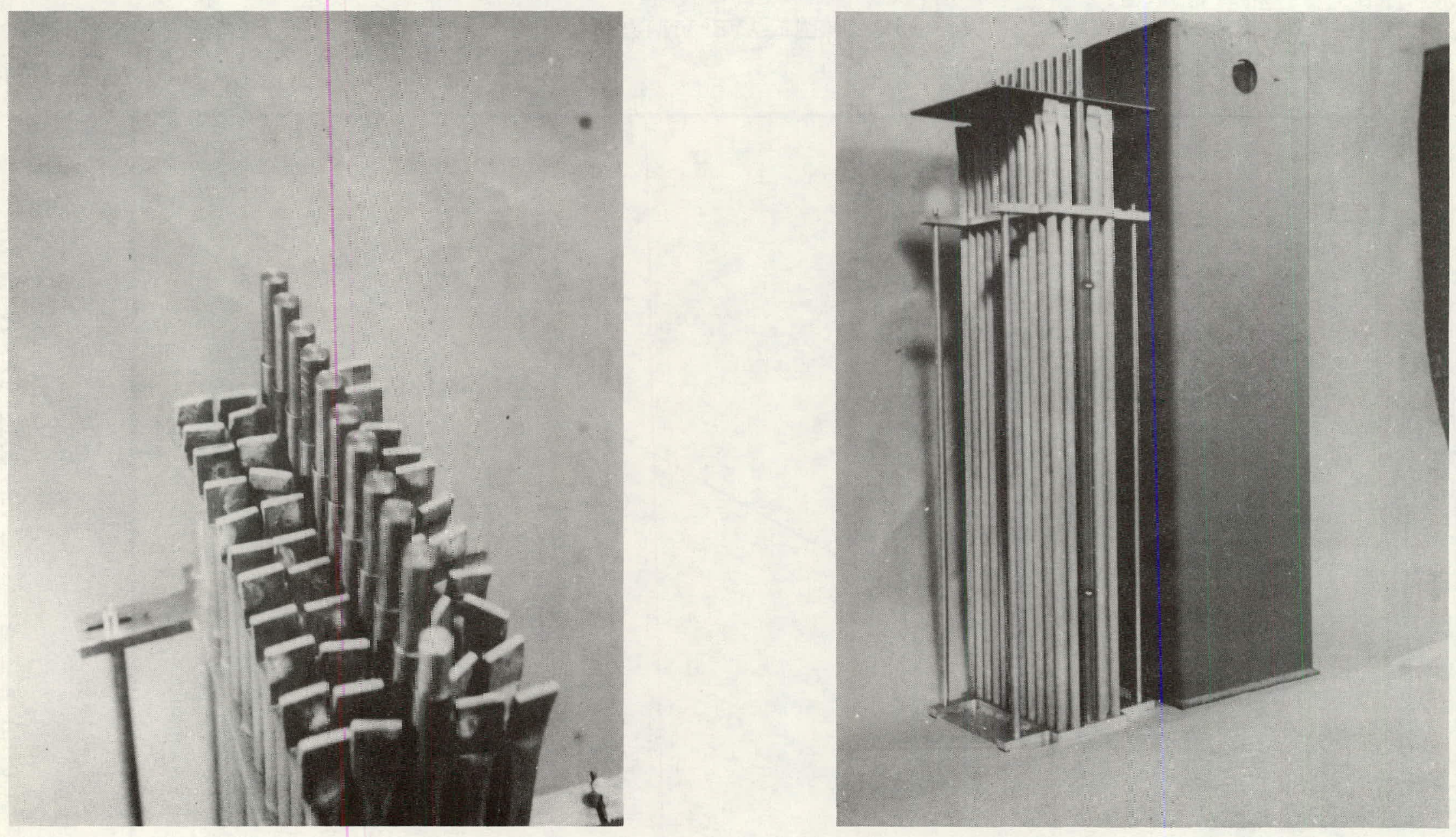

F-g. E-3 Views of Test Box and Its Fuel Rod Array 


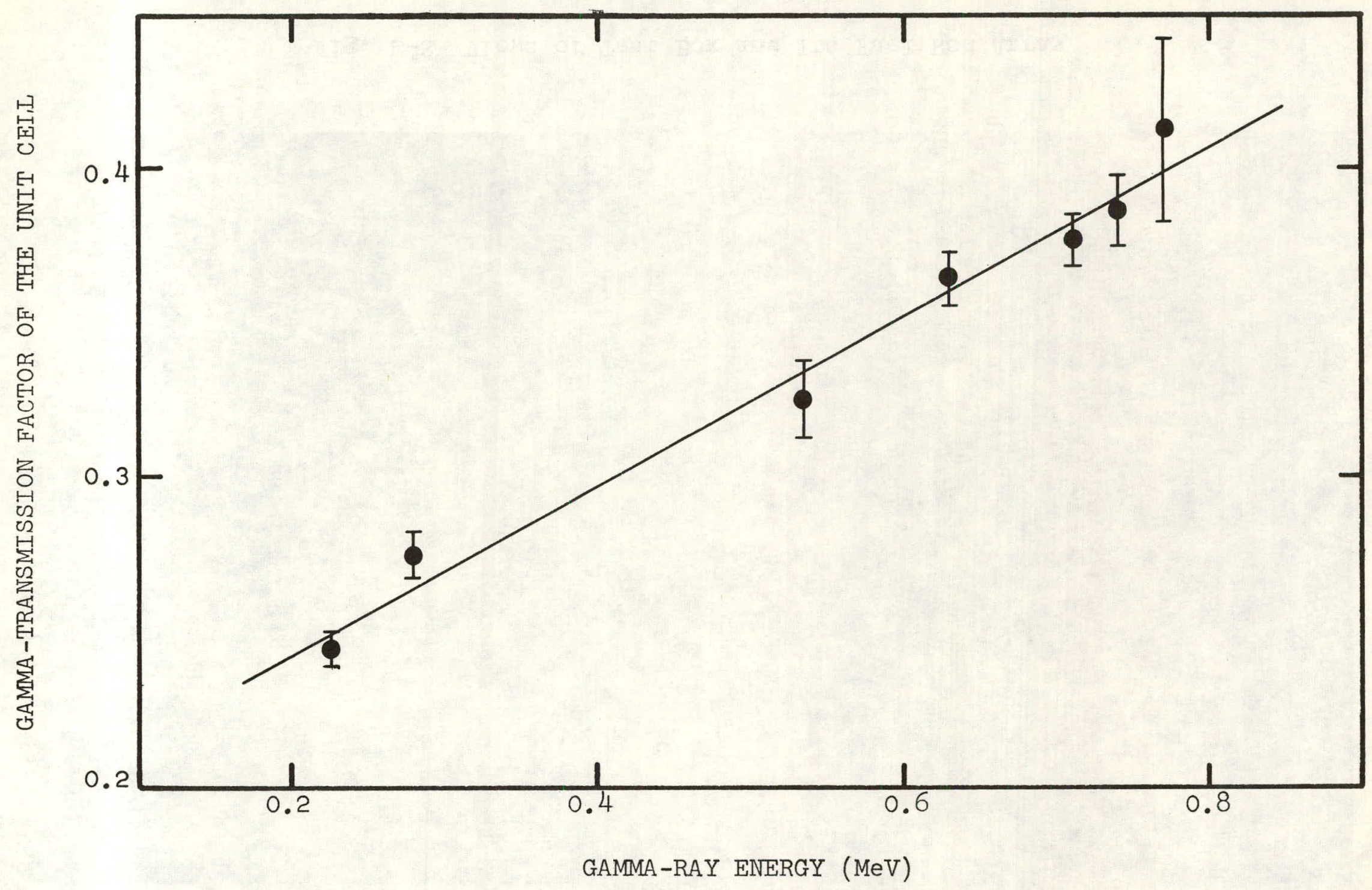

Fig. E-4 Gamma-Transmission Factor of a Unit Cell of Fuel Rods 
are compared with the measured values in the program SELFU. Input data of SELFN consists of the energy of the peak and the measured gamma-ray linear attenuation coefficients of sodium chromate, iron cladding and uranium. Input data of SELFU consists of not only the energy peak and the Iinear attenuation coefficients, but also the measured total transmission factor of the unit cell. Output data includes the self-attenuation factor of each region and the total averaged attenuation factor of the blanket, which is compared with the measured value in SELFU. 
C CALCULATION DF SELF-ATTENUATION FACTOR FOR SODIUM

C. CHROMATE IN THE BLANKET NO.2 AT MITR.

1000 CONTINUE

READ $(5,1)$ EN, XNA, XC, XU, MO

1 FORMAT $(4 \mathrm{~F}: 0.5,15)$

INPUT ARGUEMENTS

C EN - ENERGY OF THE PEAK OF INTEREST (MEV)

C XNA, XC, XU - LINEAR ATTENUATION COEFFICIENTS OF SODIUM

C CHROMATE. IRON, AND URANIUM AT. THE GAMMA-RAY ENERGY

C EN $(\Omega / C M)$, RESPECTIVELY.

WRITE $(6,90)$ EN

IO FORMAT $(\cdot 1 \cdot 14 \mathrm{X}$, 'SELF-ATTENUATION ANALYSIS FOR',F10.5, 1. MEV GAMMA').

WRITE $(6,12)$

11 FORMAT $(10 \cdot, 14 \mathrm{X}$, 'THE LINEAR ATTENUATION COEFFICIENTS $(1 / \mathrm{CM}) \cdot)$

WRITE $(6,12)$ XNA

$\$ 2$ FORMAT (\$5X, SODIUM CHROMATE ', E\$0.5)

WRITE $(6,13) \times C$

13 FORMAT (I5X, IIP.ON CLADDING , EI0.5)

WRITE $(6,94) \times U$

$c$

14 FORMAT $(15 X$, UPANIUM , E10.5)

$X I=14.304$

$\times 2=1.90 t$

$P I E=3.141592$

R.C $=0.397$

$R I=0.351$

$R=0.3175$

$R N=0.649$

$X ? A=0.643$

$\times 3=0.238$

$E L=14.79$

GAMMA $=0.0621$

$V T=(15 \cdot 24 * \times 1)-132 * P I E * R O * R O$

PAGE 1 


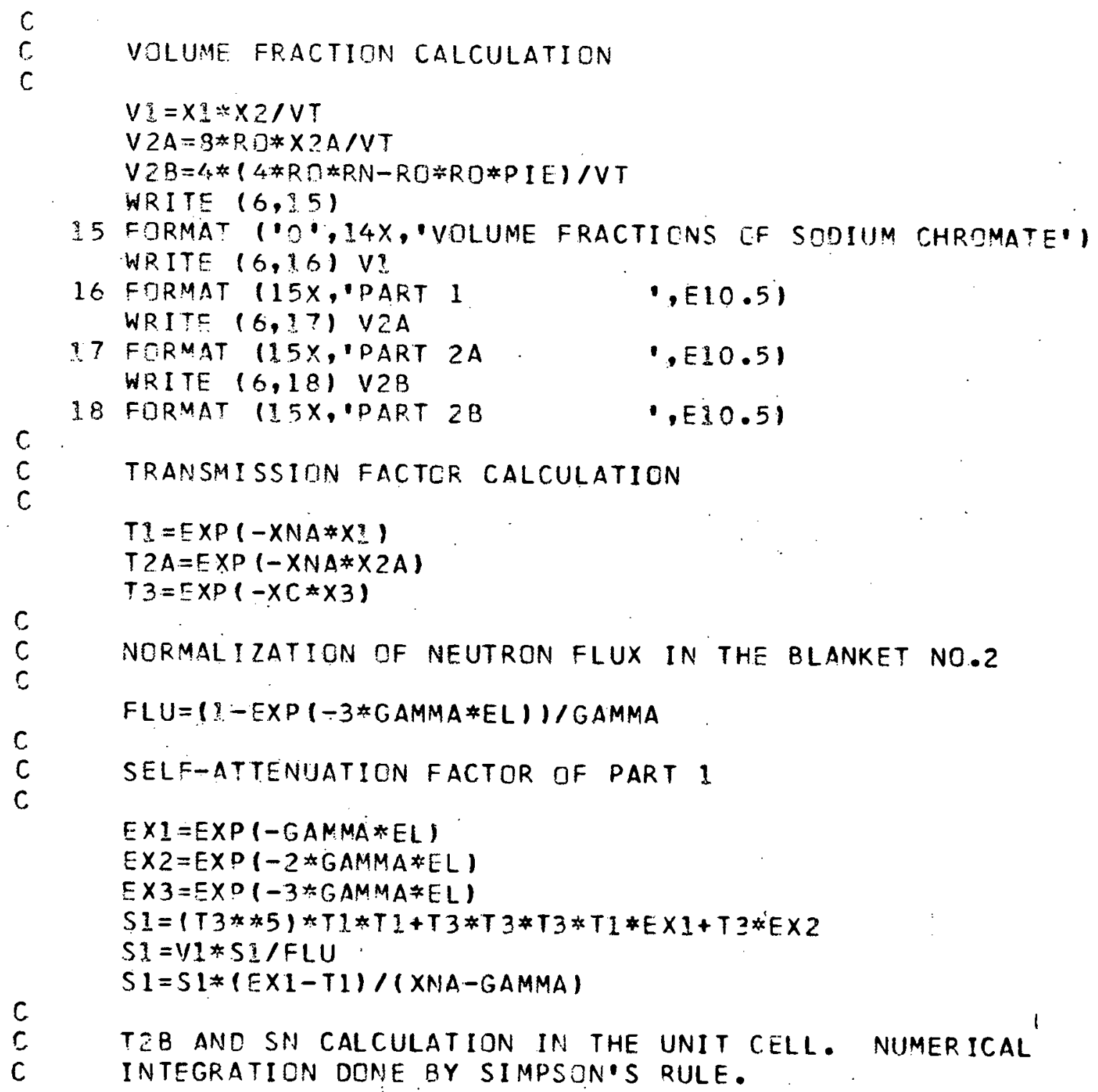

c

TEB AND SN CALCULATION IN THE UNIT CELL. NUMERICAL INTEGRATION DONE BY SIMPSON'S RULE. 
$c$

$N=41$

$Y D=R D /(N-1)$

$T 2 B=(E X P(-2 *(X N A *(R N-R O)+X C *(R C-R I)+X U * R))) * Y D / 3$

$S N=(R N-R O) *(1 .+E X P(-2 *(X C *(R O-R I)+X J * R)-X N A *(R N-R O))) * Y D /(3 * F L U)$

$11=2$

DO $200 \quad I=2, N$

$Y=Y D * F L O A T(I-1)$

$Y N D=R O * R O-Y * Y$

$Y N I=R I * R I-Y * Y$

$Y N R=R * R-Y * Y$

IF (YNO.LE.O. I YNO $=0$.

IF (YNI.LE.0.) YNI $=0$.

IF (YNR .LE.0.) YNR $=0$.

$Y N=R N-S Q R T(Y N O)$

$Y C=S Q R T(Y N O)-S Q R T(Y N I)$

$Y U=S Q R T(Y N R)$

$L=i$

DO $101 \mathrm{~J}=1, \mathrm{I}$

$203 L=(-1) * L$

$I l=I 1+2 * L$

IF $(I . E O \cdot N) \quad I I=1$

$T=1) * E X P(-2 *(X N A * Y N+X C * Y C+X U * Y U)) * Y D / 3$

$S=I 1 * Y N *(1,+E X P(-2 *(X C * Y C+X U * Y U)-X N A * Y N)) * Y D /(3 * F(U)$

$T 2 B=T 2 B+T$

$S N=S N+S$

C

100 CONTINUE

$T 2 B=T 2 B / R O$

$S N=S N / R O$

$T T=(T 2 B * * 1) * T 2 A * T 2 A * T 3 * T 3$

$E X L=(E X P(-G A M M A * X 2 A)-E X P(-X N A * \times 2 A)) /(X N A-G A M M A)$

$c$

SELF-ATTENUATION FACTOR OF PART $2 A$

$S 2 A=V 2 A * \times 2 A *(T T * T T * T T /(T 2 A * T 3)+E \times 1 * T T * T T *(T 3+1 /(T 2 A+T 3))$ 


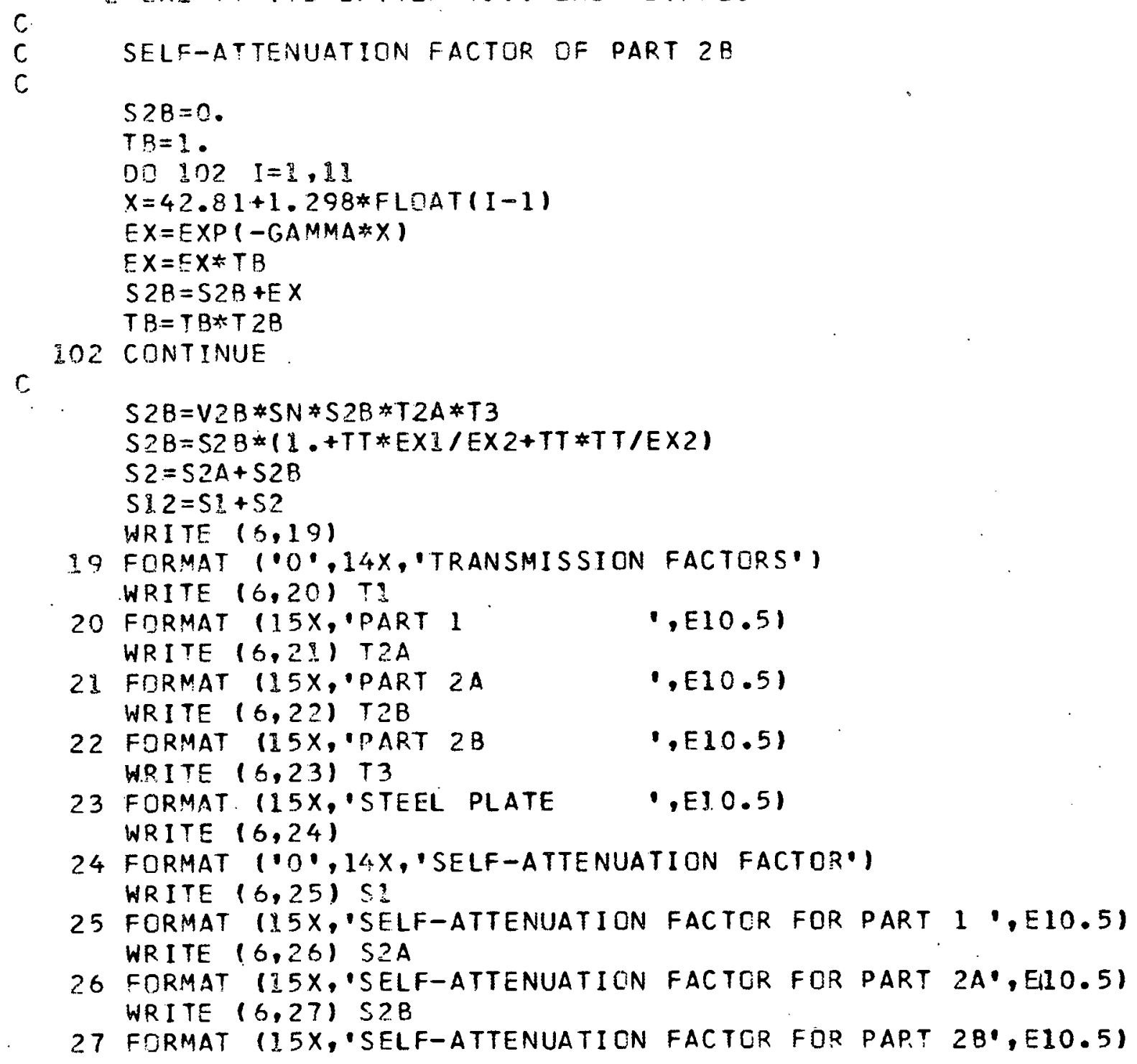


WRITE $(6,28)$ S12

28 FORMAT $(15 X$, TOTAL SELF-ATTENUATION FACTOR IF (MO.GT.O) GO TO 1000

CALL EXIT

END
,$E 10.51$ 
INPUT DATA

$\begin{array}{rrrrr}0.20980 & 0.20700 & 1.06000 & 25.50000 & 1 \\ 0.22820 & 0.20400 & 1.01400 & 20.60000 & 1 \\ 0.27760 & 0.20200 & 0.89000 & 13.00000 & 1 \\ 0.31590 & 0.19900 & 0.81500 & 20.40000 & 1 \\ 0.33430 & 0.13700 & 0.79500 & 8.51000 & 1 \\ 0.43800 & 0.18800 & 0.69500 & 4.25300 & 1 \\ 0.51500 & 0.18300 & 0.64300 & 3.17900 & 1 \\ 0.83510 & 0.15800 & 0.51200 & 1.71800 & 1 \\ 1.17300 & 0.12600 & 0.43300 & 1.29000 & 1 \\ 1.27400 & 0.12200 & 0.41400 & 1.13000 & 1 \\ 1.33250 & 0.12100 & 0.40500 & 1.04000 & 1 \\ 1.36850 & 0.12000 & 0.40100 & 1.00000 & -1\end{array}$


SELF-ATTENUATION ANALYSIS FOR 0.20980 MEV GAMMA

THE LINEAR ATTENUATION CCEFFICIENTS $(1 / \mathrm{CM})$

SODIUM CHROMATE . 20700E DO

IRCN CLADDING .10600E 31

URANIUM

$.25500 E \quad 02$

VOLUME FRACTIONS OF SCDIUM CHROMATE

PART ? $\quad .17862 E$ DC

PART 2A $.13379 E-01$

PART 2B $.14033 E-01$

TRANSMISSION FACTORS

PART $1 \quad .51771 E-01$

PART 2A $\quad .875385$ DC

PART 2B .13402E OC

STEEL PLATE $\quad .77703 E$ CO

SELF-ATTENUATION FACTOR

SELF-ATTENUATION FACTOR FGR PART $1.38197 E-02$

SELF-ATTENUATION FACTOR FOR PART 2A.28244E-04

SELF-ATTENUATION FACTOR FOR PART 2B.20712E-04

TOTAL SELF-ATTENUATION.FACTDR

$38687 E-02$ 
DUTPUT DATA

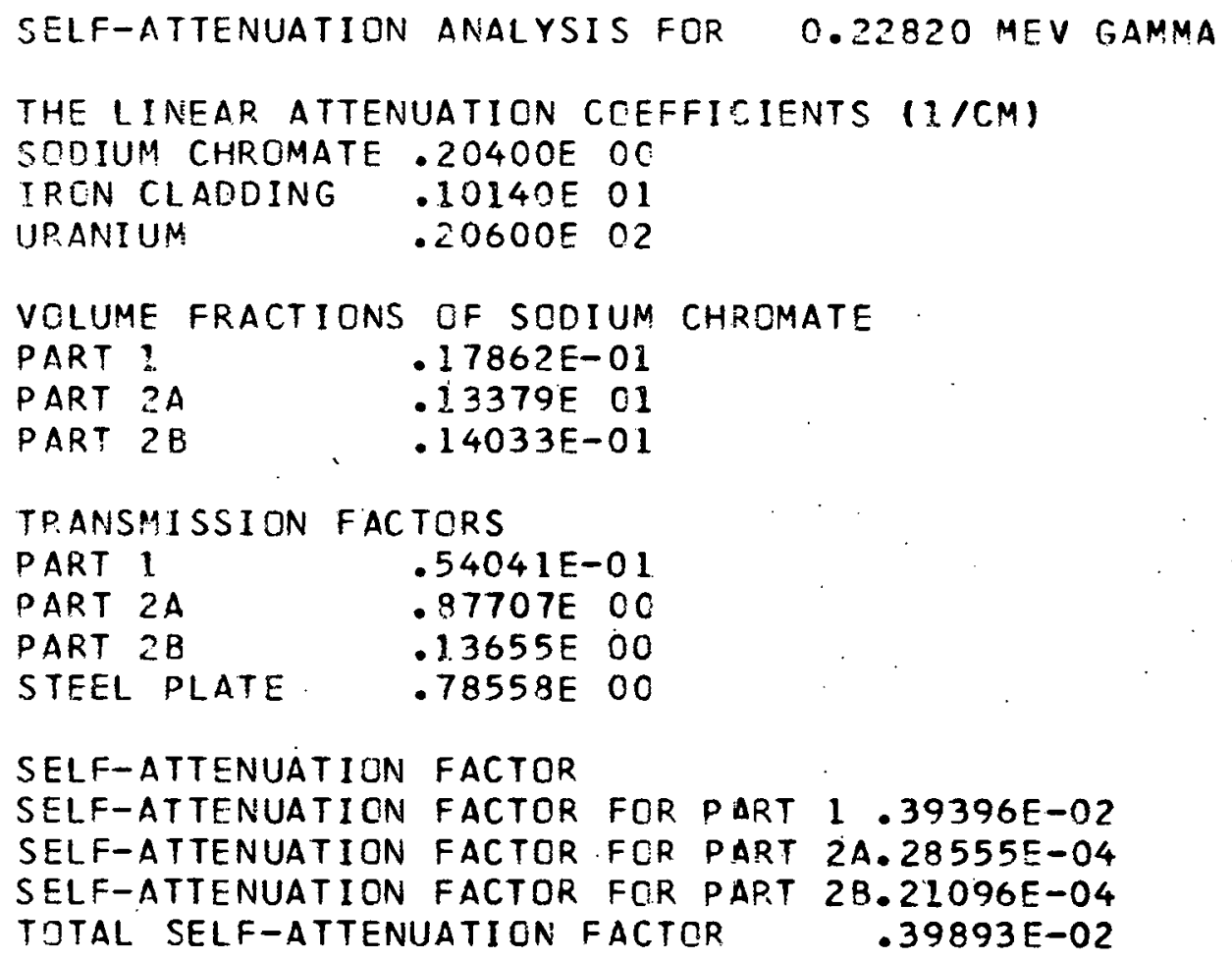


SELF-ATTENUATION ANALYSIS FOR 0.27760 MEV GAMMA

THE LINEAR ATTENUATION CDEFFICIENTS $(1 / C M)$

SODIUM CHROMATE . $20200 E$ OO

IRON CLADDING .8900OE OC

URANIUM $\quad 13000 E 02$

VOLUME FRACTIONS OF SODIUN CHROMATE

PART $1 \quad .17862 E$ OO

PART 2A $\quad .13379 E-01$

$P A R T 2 B \quad .14033 E-01$

TPANSMISSION FACTCRS

PART I $\quad .55609 E-01$

PART 2A $\quad .87820 E 00$

PART 28. $\quad .14539 E$ OO

STEEL PLATE $.80911 E$ DO

SELF-ATTENUATION FACTOR

SELF-ATTENUATION FACTOR FCR P.ART $1.41307 E-02$

SELF-ATTENUATION FACTOR. FCR PART 2A.29410E-04

SELF-ATTENUATION FACTOR FOR PART 2B.22176E-04

TOTAL SELF-ATTENUATION FACTOR $.41823 E-02$ 
SELF-ATTENUATION ANALYSIS FOR 0.31590 MEV GAMMA

THE LINEAR ATTENUATION COEFFICIENTS (I/CM)

SOOIUM CHROMATE . 19900 E 00

IRON CLADDING .8150OE OO

URANIUM $\quad .10400 E 02$

VOLUME FRACTIONS OF SODIUM CHROMATE

PART 2 .17862E 00

PART 2A $\quad .13379 E-01$

PART 2B $\quad .14033 E-01$

TRANSMISSION FACTORS

PART $1 \quad .58047 E-01$

DART 2A $.87989 E 00$

PART 2B .15375E 00

STEEL PLATE $\quad .82368 E \quad 00$

SELF-ATTENUATION FACTOR

SELF-ATTENUATION FACTOR FOR PART $1.43102 E-02$

SELF-ATTENUATION FACTOR. FCR PART 2A.29940E-04

SELF-ATTENUATION FACTOR FCR PART 2B.23016E-04

TOTAL SELF-ATTENUATION FACTOR $.43541 \mathrm{E}-02$ 
SELF-ATTENUATION ANALYSIS FOR 0.33430 MEV GAMMA

THE LINEAR ATTENUATION COEFFICIENTS (I/CM)

SODIUM CHROMATE . I9700E OO

IRON CLADDING .79500E OO

URANIUM $\quad .85100 E$ Ol

VOLUME FRACTIONS OF SODIUN CHRCMATE

PART? $\quad .27862 E$ OC

PART 2A $\quad .13379 E-01$

PART 2B .14033E-01

TRANSMISSION FACTORS

PART 1

PART $2 A$

PART $2 B$

SELF-ATTENUATION FACTOR

SELF-ATTENUATION FACTOR FCR PART $1.43823 E-02$

SELF-ATTENUATION FACTOR FCR PART 2A.30083E-04

SELF-ATTENUATICN FACTDR FCR PART 2B.2.3581E-04

TOTAL SELF-ATTENUATION FACTOR.

$.44359 E-02$ 


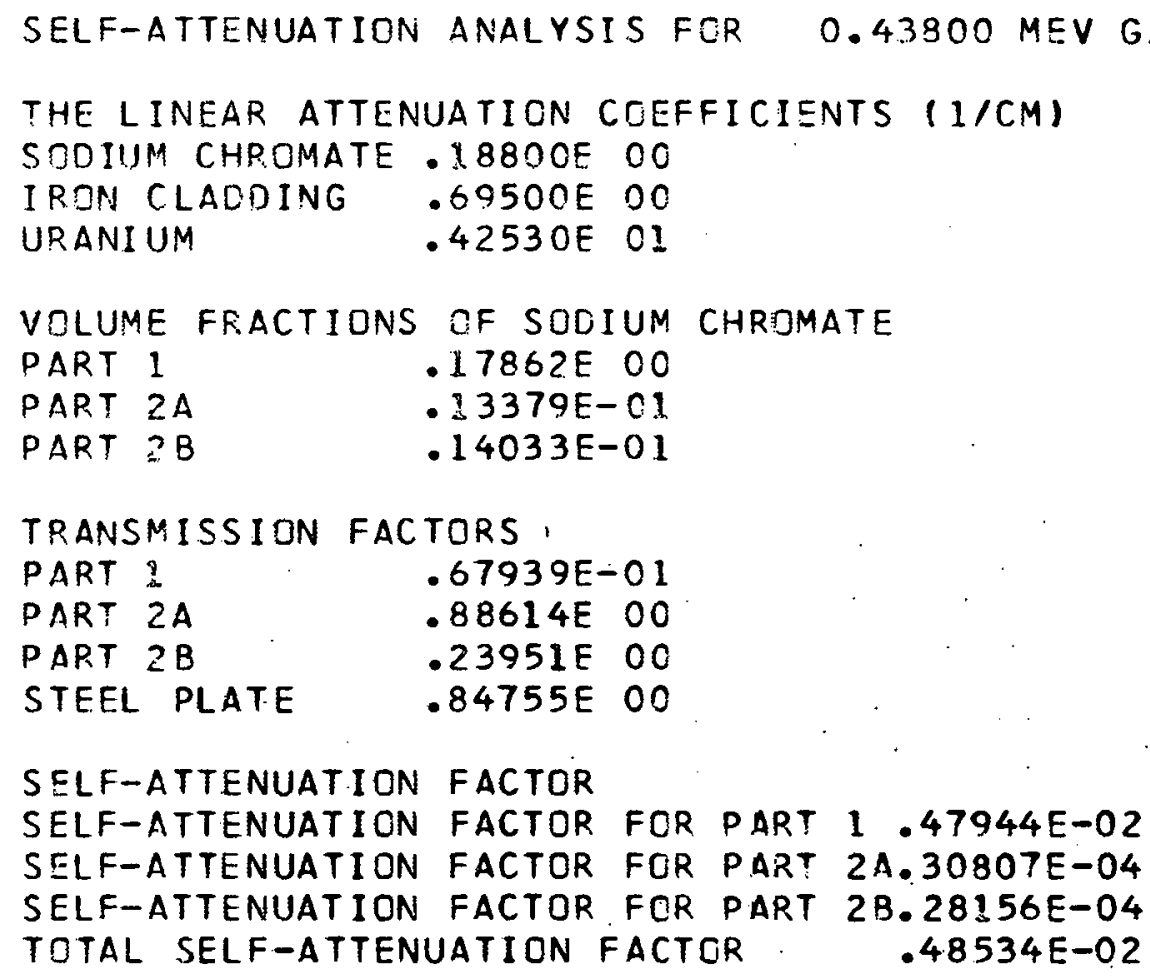


THE LINEAR ATTENUATION COEFFICIENTS $(1 / \mathrm{CM})$

SOCIUM CHROMATE . $18300 E$ OO

IRON CLADDING .64300E CO

URANIUM .31790501

VOLUME FRACTIONS OF SCDIUM CHROMATE

PART 1

PART 2A

$.17862 \mathrm{E}$ OO

PART $2 B$

$.13379 \mathrm{E}-01$

$.14033 E-01$

TRANSMISSION FACTORS

PART 2

PART $2 A$

$.72976 E-01$

PART 2B

$.88899 \mathrm{E} \quad 00$

STEEL PLATE .85810E OO

SELF-ATTENUATION FACTOR

SELF-ATTENUATION FACTCR FCR PART 1. $50460 E-02$

SELF-ATTENUATION FACTOR, FOR PART 2A.31191E-04

SELF-ATTENUATION FACTOR FCR PART 2B.31945E-04

TOTAL SELF-ATTENUATION FACTOR

$.51092 \mathrm{E}-02$ 
OUTPUT DATA

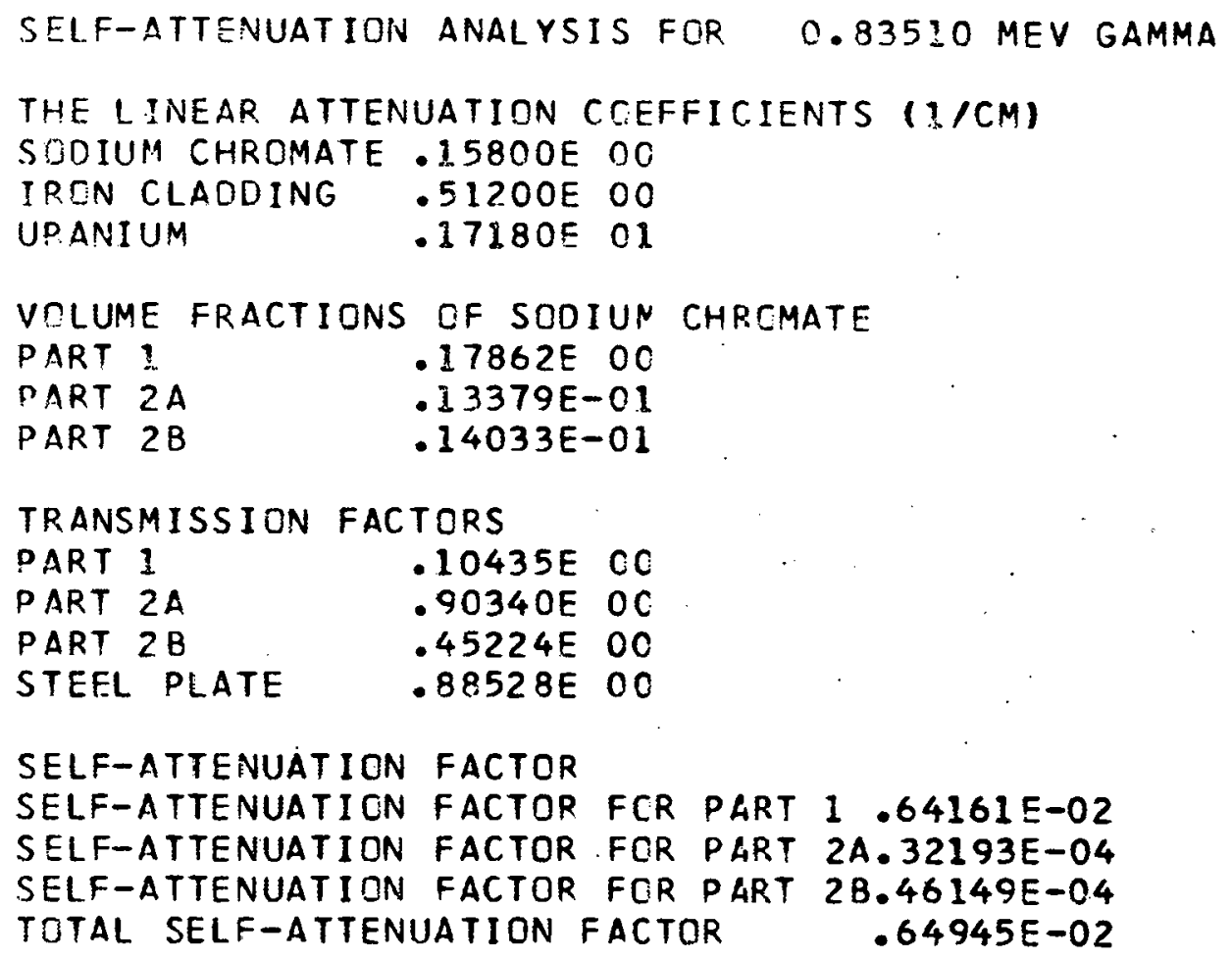




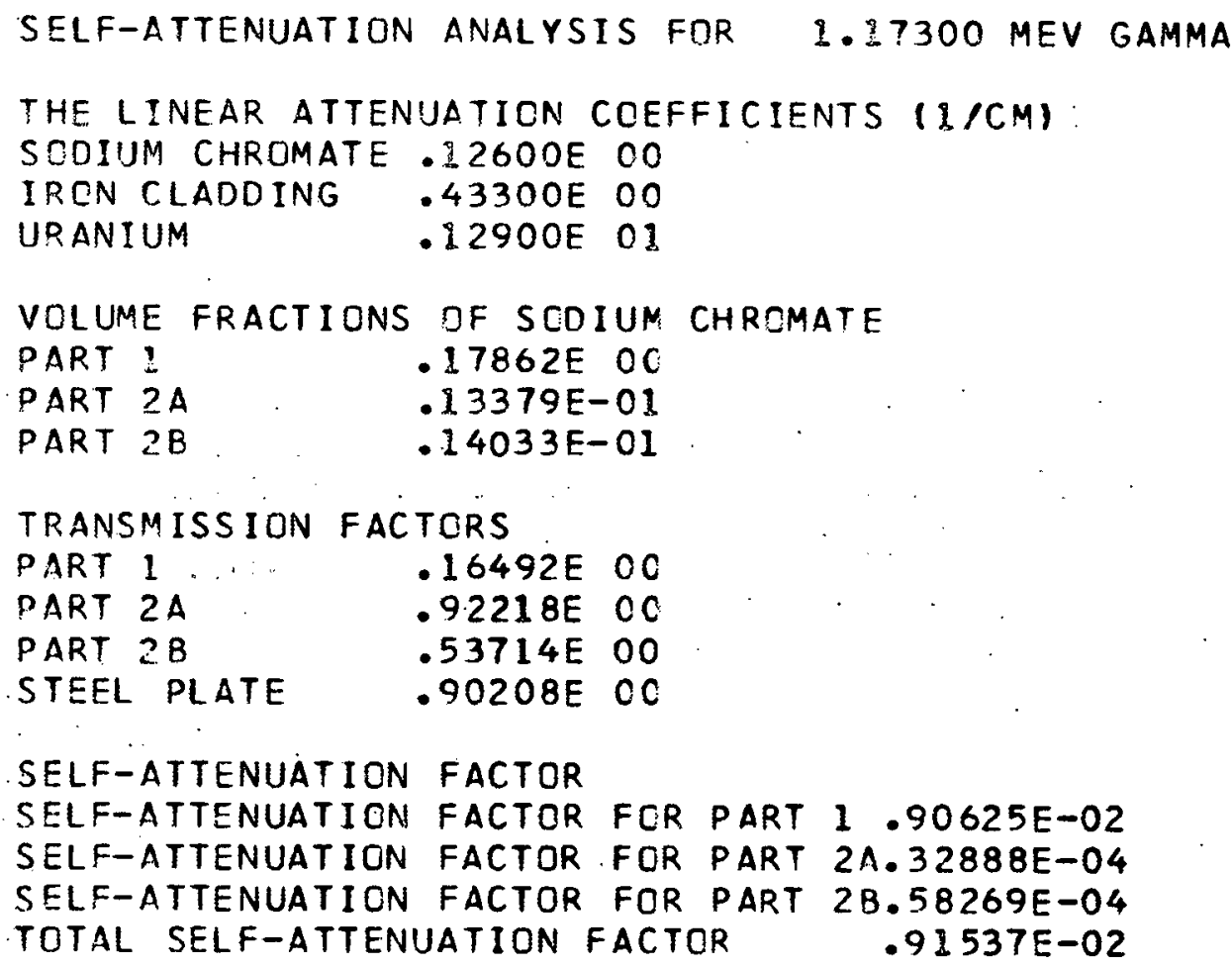


THE LINEAR ATTENUATION CCEFFICIENTS (I/CM)

SODIUM CHROMATE .I $2200 E$ OO

IRON CLADDING .414DOE OC

URANIUM

$.11300 \mathrm{E}$ O1

VOLUME FRACTIONS OF SODIUM CHROMATE

PART 1 .17862E OC

PAPT 2A $\quad .13379 E-01$

PART 2B $\quad .14033 E-01$

TRANSMISSION FACTORS

PART ? $17463 E 00$

PART 2A .92455E OC

PART 2B . .57010E OC

STEEL PLATE $\quad .90617 E 00$

SELF-ATTENUATION FACTOR

SELF-ATTENUATION FACTOR FCR PART $1.95600 E-02$

SELF-ATTENUATION FACTOR FCR PART 2A.33131E-04

SELF-ATTENUATION FACTOR FOR PART 2B.63686E-0.4

TOTAL SELF-ATTENUATION FACTOR $.96568 \mathrm{E}-02$ 
THE LINEAR ATTENUATION COEFFICIENTS $(1 /$ CM $)$

SODIUM CHROMATE .1210OE OO

IRON CLADOING .405COE CO

URANIUM $\quad .10400 E D 1$

VOLUME FRACTIONS OF SODIUM CHRDMATE

PART $1 \quad .17862 \mathrm{E}$ DO

PART 2A $\quad .13379 E-01$

PART 2B $\quad .14033 E-01$

TRANSMISSION FACTCRS

PART 1 .17715E CO

PART 2A .92515E 00

PART 2B .58923E CO

STEEL PLATE .9081lE CO

SELF-ATTENUATION FACTOR

SELF-ATTENUATION FACTOR FOR PART $1.97093 E-02$

SELF-ATTENUATION FACTOR FCR PART 2A.33288E-04

SELF-ATTENUATION FACTOR FOR PART 2B.67117E-04

TOTAL SELF-ATTENUATION FACTOR $.98097 E-02$ 
DUTPUT DATA

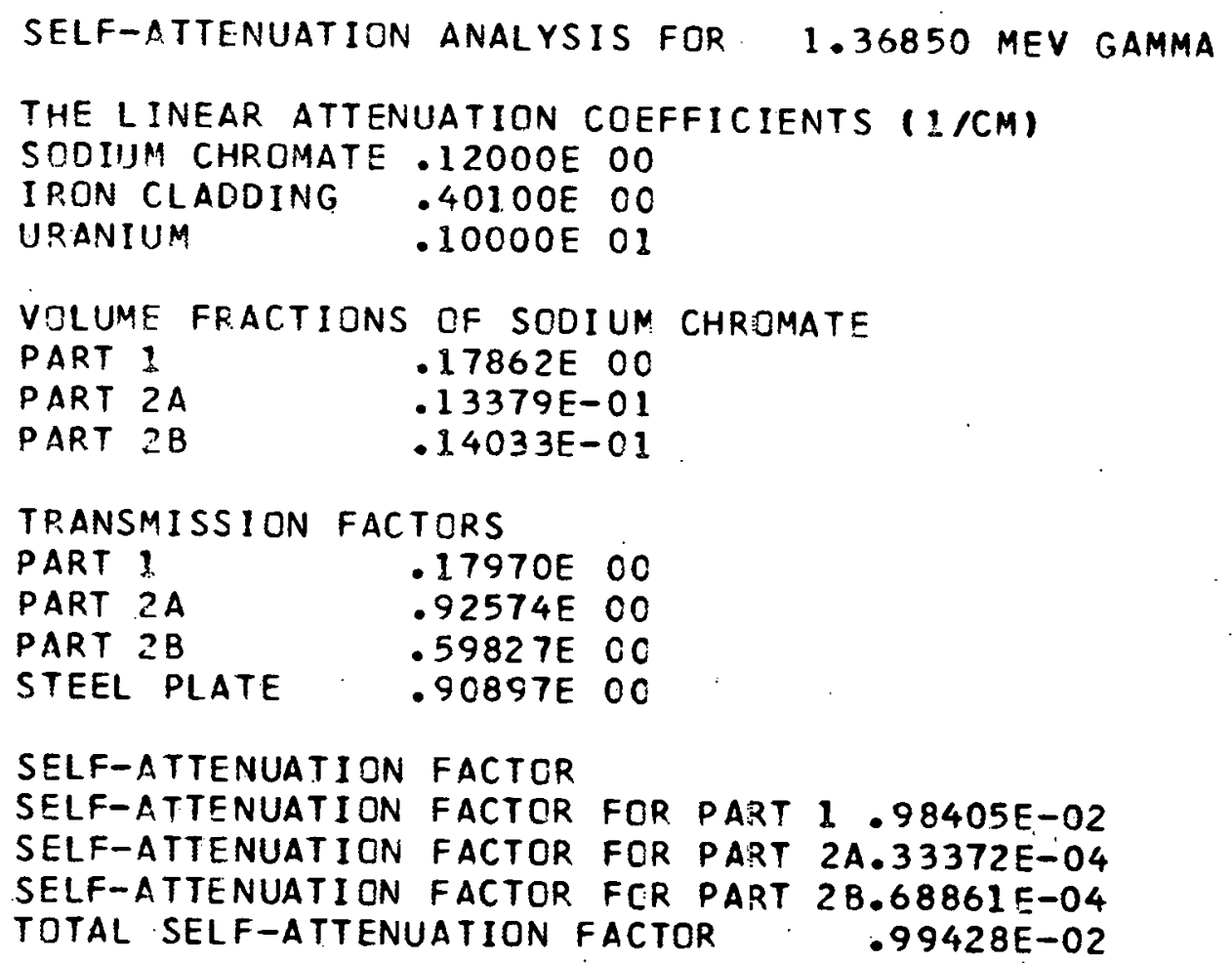


C CALCULATION OF SELF-ATTENUATION FACTOR FOR URANIUM

C

1000 CONTINUE

READ $(5,1)$ EN, XNA, XC, XU,T $2 C$, MO

1 FORMAT (5F10.5, I5)

C INPUT ARGUEMENTS

C EN - ENERGY OF THE. PEAK OF INTEREST (MEV)

C XNA, XC, XU - LINEAR ATTENUATION COEFFICIENTS OF SODIUM

C CHROMATE, IRON, AND URANIUM AT THE GAMMA-RAY ENERGY

C EN $(1 / C M)$, RESPECTIVELY.

C. T2C - MEASURED ATTENUATION COEFFICIENT OF THE CELL

$\mathrm{C}$

WRITE $(6,10) \cdot E N$

10 FORMAT $111,14 X, '$ SELF-ATTENUATION ANALYSIS. FOR', F10.5, 1. MEV GAMMA'!

WRITE $(6,11)$

11 FORMAT $(10 \cdot, 14 \dot{X}$, "THE LINEAR ATTENUATION COEFFICIENTS $(1 / C M) 1)$ WRITE $(6,12)$ XNA

12 FORMAT (15X,'SODIUM CHROMATE ,EI0.5)

WRITE $(6,13) \times C$

13 FORMAT $(15 X$, 'IRON CLADDING , E10.5) WRITE $(6,14) \quad X U$

C

14 FORMAT $115 \mathrm{X}$, UURANIUM

$\because E 10.51$

$X 1=14.304$

$P I E=3.141592$

$R O=0.397$

$R I=0.351$

$R=0.3175$

$\mathrm{RN}=0.649$

$\times 2 A=0.643$

$\times 3=0.238$

$E L=14.78$

C

GAMMA $=0.0621$ 


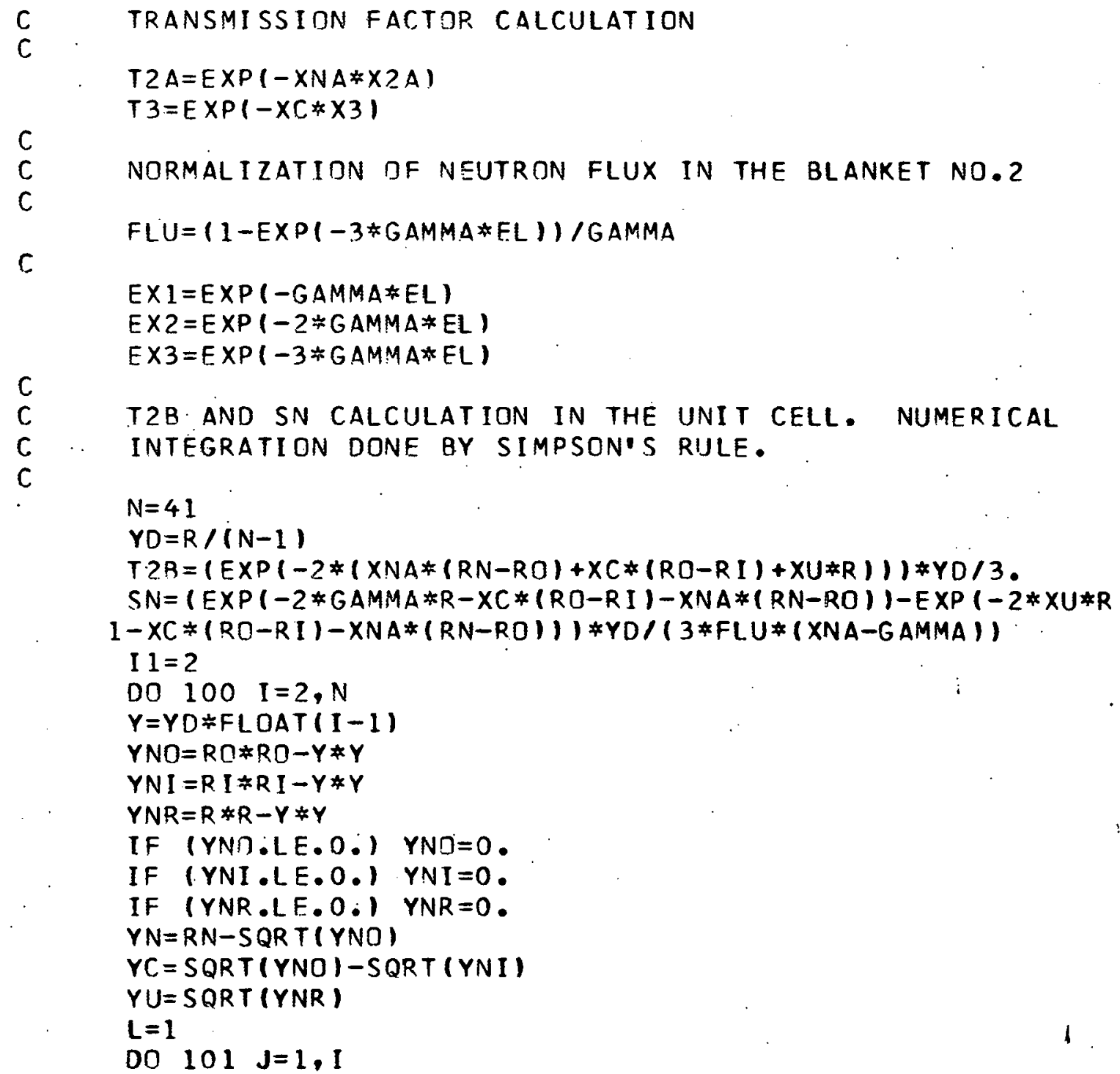
T2B AND SN CALCULATION IN THE UNIT CELL. NUMERICAL

$101 \mathrm{~L}=(-1) * \mathrm{~L}$ 
$I 1=I 1+2 * 1$

IF $(I \cdot E Q \cdot N) \quad I 1=1$

$T=11 * E X P(-2 *(X N A * Y N+X C * Y C+X U * Y U)) * Y D / 3$.

$S=(E X P(-2 * G A M M A * Y U)-X C * Y C-X N A * Y N)-E X P(-2 * X U * Y U-X C * Y C$

$1-X N A * Y N)) * Y D /(3 * F L U *(X N A-G A M M A))$

$T 2 B=T 2 B+T$

$S N=S N+S$

c

100 CONT INUE

$T 2 B=T 2 B / R$

$S N=S N / R$

$T T B=(T 2 B * 11) * T 2 A * T 2 A * T 3 * T 3$

$T T C=(T 2 C * 11) * T 2 A * T 2 A * T 3 * T 3$

$E X L=(E X P(-G A M M A * X 2 A)-E X P(-X N A * X 2 A)) /(X N A-G A M M A)$

C

SELF-ATTENUATION FACTOR OF PART $2 B$

C

$\$ 2 B=0$.

$T B=1$.

$T C=1$.

-DO $102 \quad I=1,11$

$X=42.81+1.298 *$ FLOAT $(I-1)$

$E X=E X P(-G$ AMMA*X)

$E X B=E X * T B$

$E X C=E X * T C$

$S 2 B=S 2 B+E X B$

$S 2 C=S 2 C+E X C$

$T B=T B * T 2 B$

$T C=T C * T 2 C$

c

102 . CDNTINUE

$S 2 B=S N * S 2 B * T 2 A * T 3 / 33$.

$S 2 C=S N * S 2 C * T 2 A * T 3 / 33$.

$S 2 B=S 2 B *(1+T T B * E \times 1 / E \times 2+T T B * T T B / E \times 2)$

$S 2 C=S 2 C *(1+T T C * E \times 1 / E \times 2+T T C * T T C / E \times 2)$ 
WRITE $(6,19)$

19 FORMAT $1:, 14 X$, 'TRANSMISSION FACTORS'

WRITE $(6,21)$ T2A

21 FORMAT $(15 X, 1$ PART $2 A$

WRI TE $(6,22)$ T2B

22 FORMAT (15X, 'PART 2B (CALCULATED)', E10.5)

WRITE $(6,25)$ T2C

25 FDRMAT (15X,'PART 28 (MEASURED) ', E10.5)

WR.ITE $(6,23)$ T3

23 FORMAT $(15 x$, STFEL PLATE, E10.5)

WRITE $(6,24)$

24 FORMAT ('0', 14X, 'SELF-ATTENUATICN FACTOR')

WRITE $(6,28)$ S2B

28 FORMAT $(15 X, '$ 'SELF-ATTENUATION FACTOR (CALCULATED)', E10.5) WRITE $(6,26)$ S?C

26 FORMAT (15X,' SELF-ATTENUATION FACTOR (MEASURED) ',E10.5)

IF $(M O . G T .0)$ GO TO 1000

CALL EXIT

END 
INPUT DATA

$\begin{array}{rrrrrr}0.20980 & 0.20700 & 1.06000 & 25.50000 & 0.24600 & 1 \\ 0.22820 & 0.20400 & 1.01400 & 20.60000 & 0.25100 & 1 \\ 0.27760 & 0.20200 & 0.89000 & 13.00000 & 0.26450 & 1 \\ 0.31590 & 0.19900 & 0.81500 & 10.40000 & 0.27490 & 1 \\ 0.33430 & 0.19700 & 0.79500 & 8.51000 & 0.27990 & 1 \\ 0.43800 & 0.18800 & 0.69500 & 4.25300 & 0.30810 & 1 \\ 0.51500 & 0.18300 & 0.64300 & 3.17900 & 0.32910 & 1 \\ 0.83510 & 0.15800 & 0.51200 & 1.71800 & 0.41620 & -1\end{array}$


DUTPUT DATA

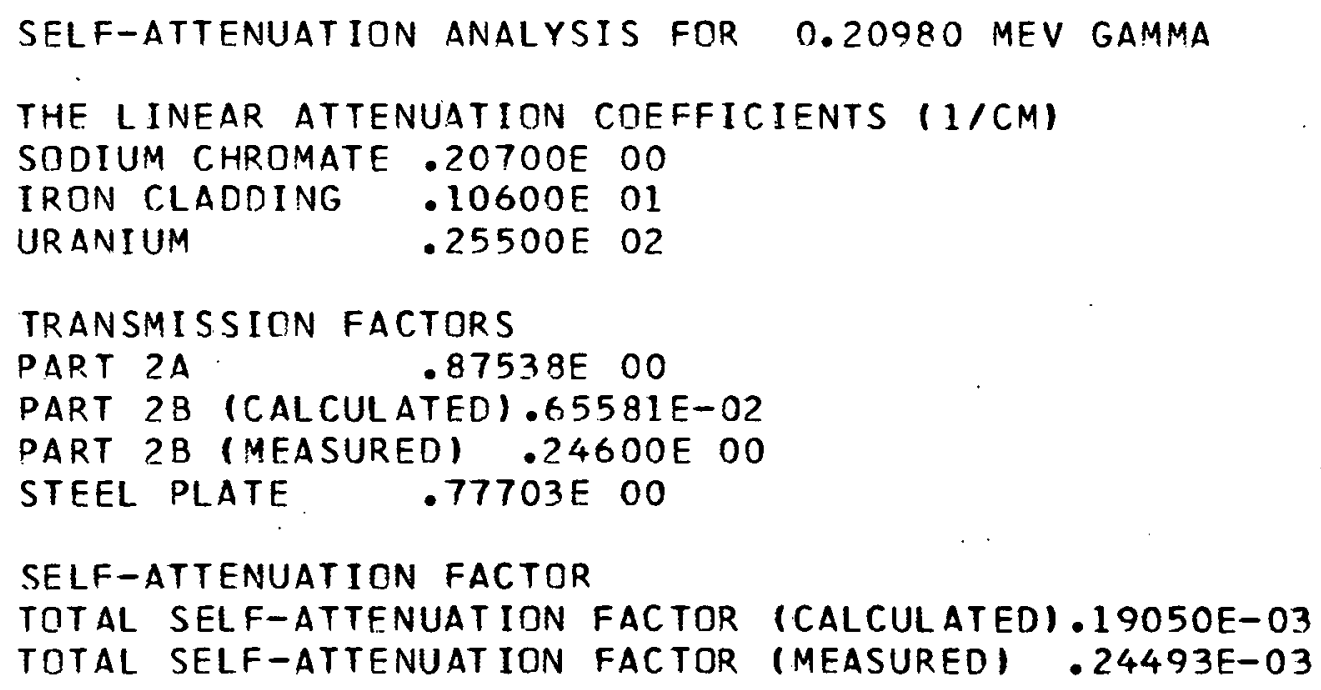


DUTPUT DATA

SELF-ATTENUATION ANALYSIS FOR 0.22820 MEV GAMMA

THE LINEAR ATTENUATION COEFFICIENTS $(1 / \mathrm{CM})$

SODIUM CHROMATE .20400E OO

IRON CLADDING . $10140 E$ OI

URANIUM $\quad .20600 E 02$

TRANSMISSION FACTORS

PART 2A $\quad .87707$ OO

PART 2B (CALCULATED) .76489E-02

PART 28 (MEASURED) .25100E 00

STEEL PLATE $\quad .78558 E$ O0

SELF-ATTENUATION FACTOR

TOTAL SELF-ATTENUATION FACTOR (CALCULATED).19766E-03

TOTAL SELF-ATTENUATION FACTOR (MEASURED) .25541E-03 
SELF-ATTENUATION ANALYSIS FOR $0.27760 \mathrm{MEV}$ GAMMA

THE LINEAR. ATTENUATION COEFFICIENTS $(1 / \mathrm{CM})$

SODIUM CHROMATE .20200E OO

IRON CLADDING $.89000 E$ OO

URANIUM $\quad .13000 E$ OO

TRANSMISSION FACTORS

PART 2A $\quad .87820$ OE DO

PART 2B (CALCULATED).13941E-01

PART 2B (MEASURED). $.26450 E$ O0

STEEL PLATE $\quad .80911 \mathrm{E} 00$

SELF-ATTENUATION FACTOR

TOTAL SELF-ATTENUATION FACTOR (CALCULATED).20791E-03

TOTAL SELF-ATTENUATION FACTOR (MEASURED) .27148E-03 
SELF-ATTENUATION ANALYSIS FOR 0.31590 MEV GAMMA

THE LINEAR ATTENUATION COEFFICIENTS $(1 / \mathrm{CM})$

SODIUM CHROMATE . $19900 E$ OO

IRON CLADDING $.81500 E$ OO

URANIUM $\quad .10400 E$ OO

TRANSMISSION FACTORS

PART 2A $\quad .87989 E \quad 00$

PART 2B (CALCULATED).21088E-01

PART 2B (MEASUREO) .27490E OO

STEEL PLATE $\quad .82368 E \quad 00$

SELF-ATTENUATIDN FACTOR

TOTAL SELF-ATTENUATION FACTOR (CALCULATEO).21732E-03

TOTAL SELF-ATTENUATION FACTOR (MEASURED) $.28549 E-03$ 
SELF-ATTENUATION ANALYSIS FOR 0.33430 MEV GAMMA

THE LINEAR ATTENUATION COEFFICIENTS $(1 / C M)$

SODIUM CHROMATE . $19700 E$ OO

IRON CLADDING $.79500 E$ OO

URANIUM $\quad .85100 F 01$

TRANSMISSION FACTORS

PART 2A $\quad 88102 \mathrm{E} 00$

PART 2B (CALCULATED).31573E-01

PART 2B (MEASURED) .27990E OO

STEEL PLATE $\quad .82761$ E 00

SELF-ATTENUATION FACTOR

TOTAL SELF-ATTENUATION FACTOR (CALCULATED).22146E-03

TOTAL SELF-ATTENUATION F.ACTOR (MEASURED) .28986E-03 
DUTPUT DATA

SELF-ATTENUATION ANALYSIS FOR 0.43800 MEV GAMMA

THE LINEAR ATTENUATION CJEFFICIENTS $(1 / C M)$

SODIUM CHROMATE . 18800 DO

IRON CLADDING .6950OE DO

URANIUM $\quad .42530$ JI

TRANSMISSION FACTORS

PART 2A $\quad .88614 E$ DO

PART 2B (CALCULATED).122DOE OO

PART 2B (MEASURED) .30810E 00

STEEL PLATE $\quad 84755 E$ DO

SELF-ATTENUATION FACTOR

TOTAL SELF-ATTENUATION FACTOR (CALCULATED).23827E-03

TOTAL SELF-ATTENUATION FACTOR (MEASURED) .29542E-03 
SELF-ATTENUATION ANALYSIS FOR 0.51500 MEV GAMMA

THE LINEAR ATTENUATION COEFFICIENTS $(1 / \mathrm{CM})$

SODIUM C.HROMATE . $18300 E$ OO

IRON CLADDING :64300E 00

URANIUM $\quad .31790 E$ OI

TRANSMISSION FACTORS

PART 2A $\quad .88899 E \cdot 00$

PART 2B (CALCULATED).19028E 00

PART 2B (MEASURED) .32.910E OO

STEEL PLATE $\quad .85810$ O. O0

SFLF-ATTENUATION FACTOR

TOTAL SELF-ATTENUAT ION FACTOR (CALCUL ATED) $.24585 E-03$

TOTAL SELF-ATTENUATION FACTOR (MEASURED) .29106E-03 
OUTPUT DATA

SELF-ATTENUATION ANALYSIS FOR 0.83510 MEV GAMMA

THE LINEAR ATTENUATION COEFFICIENTS $(1 /$ CM)

SODIUM CHROMATE . $15800 E$ OO

IRON CLADDING . .51200E DO

URANIUM $\quad .17180 E$ OI

TRANSMISSION FACTORS

PART 2A $90340 E$ OO

PART 2B (CALCULATED).37496E 00

PART $2 B$ (MEASURED) $.41620 E$ OO

STEEL PLATE $.88528 E 00$.

SELF-ATTENUATION FACTOR

TOTAL SELF-ATTENUATION FACTOR (CALCULATED).29920E-03

TOTAL SELF-ATTENUAT ION FACTOR (MEASURED) .31770E-03 


\section{Appendix F \\ Computer Program SPECT}

The computer code SPECT calculates the neutron energy spectra from Blanket. Nn. 2 given the broadened Ge 691.4 $\mathrm{KeV}$ spectral line as input. The subroutine SMOOTH smooths the input data and the subroutine SPLINE interpolates among the given data using the cubic spline function (G9). The standard broadened spectrum $691.4 \mathrm{KeV}$ for $\mathrm{Cf}-252$ (Run No. 91) and the broadened $\overline{6} 91.4 \mathrm{KeV}$ spectrum from Blanket No. 2 (Run No. 105) were used to unfold the blanket neutron spectrum. Typical input and output data immediately follow the code listing. Input data consist of the number of runs, the number of channels the minimum channel at which the analysis begins, the degree of fredom of smoothing, the energy per channel and the spectral data for the standard source run and the same input data for the blanket run follow. 
C NEUTRON ENERGY SPECTRA ANALYSIS FROM 691.4 KEV INTERNAL

C. CONVERSION LINE.

C

REAL DATA $(8200), E(100), W(100)$, FLUX(100), CFLUX(100),

$1 \times(500), Y(500), T(500), D E L Y(500), S 2(500), S 3(500), S S(500), B(500)$

WR ITE $(6,25)$

25 FORMAT $1 ' 1 \cdot, 14 \mathrm{X}$, 'THE ANALYSIS OF STANDARD NEUTRON SOURCE (F-252')

1000 CONT INUE

READ $(5,1)$ NRUN, NCHAN, IMAX,LT, IDIST,EDELT, LIM

1 FORMAT $(515, F 10.5,15)$

READ $(5,2)$ (DATA(I), I=1, NCHAN)

C

2 FORMAT $(7 X, 7(F 6.0,1 X) /(8(F 6.0,1 X)))$

BACKGROUND SUBTRACTION

DO $200 \quad I=1$, NCHAN

DATA (I) = DATA (I)-DATA (NCHAN)

IF (DATAI I ).LE.O.) NCHAN = I

C

200. CONTINUE

C FORMING THE VECTOR FOR THE MEASURED 691.4 KEV SPECTRA.

C.

QVALUE $=691.4$

GEMASS $=72.0$

EMIN $=$ QVALUE $/(1.0+$ GEMASS $)$

EMIN $=-1.77196+1.16293 * A L$ OG (EMIN)

$E M I N=\operatorname{EXP}(E M I N)$

C

$E M I N=E M I N+I D I S T * E D E L T$

WRITE $(6,10)$ NRUN, NCHAN

10 FORMAT $1 \cdot 0 \cdot, 14 X, '$ PARAMETERS AND INPUT DATA FOR RUN', I5,

$11 X, I 5, '$ CHANNELS'I

WRITE $(6,11)$ IMAX

11 FORMAT $(10,14 \times$, 'LOW CHANNEL NUMBER WHICH BEGINS ANALYSIS', I5) WRITE $(6,13)$. EDELT

13 FORMAT (15X, ENERGY PER CHANNEL NUMBER (KEV),;F7.5)

CALL SMOOTH (IMAX,NCHAN, IDIST, OATA, W, FLUX, NOUT) 
$N=2 * I D I S T *(N O U T-1)+1$

DO $204 I=1, N$

C

$204 \mathrm{~T}(\mathrm{I})=$ FLOAT (IDIST $+I-1)$

CALL SPLINE (FLUX,W,T,DATA,NOUT,N)

C

NCHAN $=$ IMAX $+(2 * N O U T-1) *$ I D IST

IMAX = IMAX + IDIST

DO $109 \quad I=1, N$

$109 W(I)=D A T A(I) \div 100$

DO $205 I=1, N$

$K=I+I M A X-1$

DATA $(K)=W(I)$

205 CONT INUE

NOUT $=($ NCHAN-IMAX) /IOIST

WRITE $(6,12)$ NOUT

12 FORMAT (15X,'DIMENSION OF.MATRIX', I5)

C

DO $100 \quad I=1$, NOUT

$I U=I M A X+I D I S T * I$

$I L=I M A X+I D I S T *(I-1)$

$I P=I U-I D I S T / 2$

$E(I)=E M I N+($ I P-I MAX) *EDELT

$E(I)=1.52370+0.85990 * A L O G(E(I)$

$E(I)=E X P(E(I) / / 1000$.

$W T=D A T A(I L)$

$I L=I L+1$

$I 1=2$

$I K=2$

DO $101 \mathrm{~J}=1 \mathrm{~L}, \mathrm{IU}$

$\mathrm{L}=1$

DD $102 \mathrm{~K}=1, \mathrm{IK}$

$102 \mathrm{~L}=(-1) * \mathrm{~L}$

$I 1=I 1+2 * L$

$=I K+1$

IF $(J \cdot E Q \cdot I U) \cdot I I=1$ 
$W T=W T+D A T A(J) * I 1$

101 CONT INUE

$W(I)=W T /(3 *$ IDIST)

$c$

100 CONTINUE

C

FORMING THE RESPONSE MATRIX

I $T=1$

DO $103 \mathrm{I}=1$, NOUT

DO $104 \mathrm{~J}=\mathrm{I}$, NOUT

I $P=$ IMAX+IDIST*J-IDIST/ 2

$E R=E M I N+(I P-I M A X) * E D E L T$

$E P=1.52370+0.85990 * A L O G(E R)$

$E P=E \times P(E P) / 1000$

$E P=(E P *(1 .+G E M A S S)+Q V A L U E / 1000) *.(E P *(1 .+G E M A S S)+Q V A L U E / 1000.1 /$

$1(4 * G E M A S S * E P)$

DELTA $=S Q R T(1 .-1$ GEMASS+1.)*QVALUE/(SEMASS*EP*10OC) )

$E L=G E M A S S *(D E L T A+1) *.(D E L T A+1) /.(($ IEMASS +1.$) *(G E M A S S+1)$.

$E L=E R / E L$

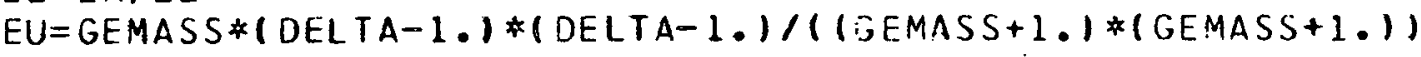

$E U=E R / E U$

EDIST $=(E U-E L) / N O U T$

$\mathrm{NI}=\mathrm{NOU} \cdot \mathrm{T}+1$

$C T=($ GENASS $+1.1 *($ GEMASS +1.$) /(4 *$ GEMAS $S * E L * D E L T A)$

$I \mathrm{I}=2$

DO $105 K=2, N I$

$E M=E L+E D I S T *(K-1)$

$L=1$

DO $106 \mathrm{KI}=1, \mathrm{~K}$

$106 \mathrm{~L}=(-1) * \mathrm{~L}$

$I I=I l+2 * L$

IF (K.EQ.NI) $I 1=1$

$C=($ GEMASS + 1.$) *($ GEMASS + 1.$) /(4 * G E M A S S * E M * D E L T A)$

$C T=C T+C$

105 CONTINUE

DATAI I T $=C T * E D I S T / 3$ 
$\mathrm{I} T=\mathrm{IT}+1$

104 C.ONTINUE

$E(I)=(1000 * E(I) *(1 .+G E M A S S)+Q V A L U E) *(1004 * E(I) *(1 .+G E M A S S)$

$1+Q$ VALUE $/ /(4000 *$ GEMASS*E(I))

FLUX (I) $=W(I)$

C

WRITE $(6,18)$

18 FORMAT ('O',14X, 'THE ENERGY ARPAY OF NEUTPON IN KEV')

WR ITE $(6,17)(E(I), I=1$, NOUT)

17 FORMAT $(1 \times, 6 E 12.5)$

IF ILIM.EQ.1) GO TO 2000

C

C SOLVING. THE MATRIX EQLATION BY THE ITERATIVE UNFOLOING METHOD

$\mathrm{C}$

DO $108 \quad I=1$, NOUT

c

108 FLUX(I) $=$ W (I)/DATA(I)

WR ITE $(6,21)$

21 FORMAT $(: 0,14 X$, 'THE MEASURED NEUTREN FLUX OF CF-252')

WRITE $(6,17) \quad(F L U X(I), I=1$, NOUT)

C

C CORRECTION FUNCTION FOR RESPONSE MATRIX

C

DO $111 \mathrm{I}=1$, NOUT

$W(I)=S Q R T(E(I)) * E X P(-E(I) / 1390$.

CFLUXI I $=$ FLUX(I)/W(I)

$X(I)=E(I)$

111 CONTINUE

WRITE $(6.23)$

23 Format $(' 1,14 X$, the Calculated NeUtron flux of CF-252')

WRITE $(6,17)$ (WII), I=1, NOUT)

WRITE. $(6,24)$

24 FORMAT $1: 0^{\prime}, 14 X$, 'THE CORRECTION DIAGCNAL MATRIX FORI THE SYSTEM') WRITE $(6,17)$ (CFLUX(I), I =1, NOUT)

C

PAGE 
ECMIN=E(1)

ECMAX $=E$ (NOUT)

C

NCF $=$ NOUT

WRITE $(6,26)$

C

26 FORMAT $111,14 X$, 'THE ANALYSIS OF UNKNOWN NEUTRON ENERGY SPECTRA')

$\mathrm{C}$ $\mathrm{C}^{200}$
GO TO 1000

2000 CONTINUE

C

CALL SPLINE (X,CFLUX,E,SS,NCF,NOUT)

DO $116 \quad I=1$, NOUT

DATA $(I)=$ DATA $(I) * S S(I)$

FLUX(I) $=W(I) / D A T A(I)$

116 CONTINUE

WRITE $(6,19)$

19 FORMAT $(10,14 \mathrm{X}$, 'THE CORRECTED DIAGCNAL RESPUNSE MATRIX') WRITE $(6,17)$ (DATAII), I=1, NOUT)

WRITE $(6,27)$

27 FORMAT $1 ' 1 ', 14 x$, 'THE NEUTRON ENERGY SPECTRA FROM BLANKET'' WRITE $(6,17)$ ( $F L U X(I), I=1$, NOUT)

CALL EXIT

END 
REAL DATA $(500), W(500), \times(500)$

NOUT $=(N C H A N-I M A X) /(? *$ ID IST $)$

DC: 3. I $=1$, NOUT

IU $=$ IMAX $+2 * I D I S T * I$.

$I L=I M A X+2 * I D I S T *(I-1)$

WT $=$ DATAR ILI/1T/O

$I L=I L+1$

I $1=2$

$\mathrm{I} K=2$

DO $2 \mathrm{~J}=\mathrm{IL}, \mathrm{IU}$

$\mathrm{L}=1$

DO $3 \mathrm{~K}=1, \mathrm{IK}$

$3 L=(-1) * L$

$I \mathrm{I}=\mathrm{I} 1+2 * \mathrm{~L}$

$I K=I K+1$

IF (J.EO. IU) I $1=1$

$W T=W T+D A T A(J) * I 1 / 100$.

2. CONTINUE

$W(I)=W T /(6 *$ IDIST)

$X(I)=F L O A T((2 * I-1) *$ IOIST)

1 CONTINUE

RETURN

END 
SUBRCUTINE SPLINF $(X, Y, T, S S, N, M)$

C NUMERICAL INTERPOLATION GY USING SPLINE FUMCTIONS

REAL X(500),Y(500),T(500), )ELY(500),S2(500), S3(50), I.SS $(500), 8 .(500)$

$N$

$M$

EPSLN

$X(I), Y(I)$

2. $\mathrm{NI}=\mathrm{N}-1$

3 DO $51 \quad I=1, N 1$

$S S(I)=X(I+I)-X(I)$

51 DELY $(I)=(Y(I+I)-Y(I)) / S S(I)$

4 DO $52 \quad \mathrm{I}=2, \mathrm{~N} 1$

$S S 1=S S(I-1)+S S(I)$

$S 3(I)=.5 * S S(I-1) / S S I$

DEL SQY $=(D E L Y(I)-D E L Y(I-1)) / S S 1$

$S 2(I)=2 . * D E L S Q Y$

$52 B(I)=3$. *DEL SQY

$S 2(1)=0$.

$S 2(N)=0$.

OMEGA $=1.0717968$

5 ETA $=0$.

6 DO $10 \quad I=2, N 1$

$7 W=(B(I)-S 3(I) * S 2(I-1)-(.5-S 3(I)) * S ?(I+1)-S 2(I)) * O A E G A$

8 IF (ABS(W)-ETA) $10,10,9$

$9 E T A=A B S(W)$

$10 S 2(I)=\$ 2(I)+W$

EPSLN=0.0001

13 IF (ETA-EPSLN) $14,5,5$ 


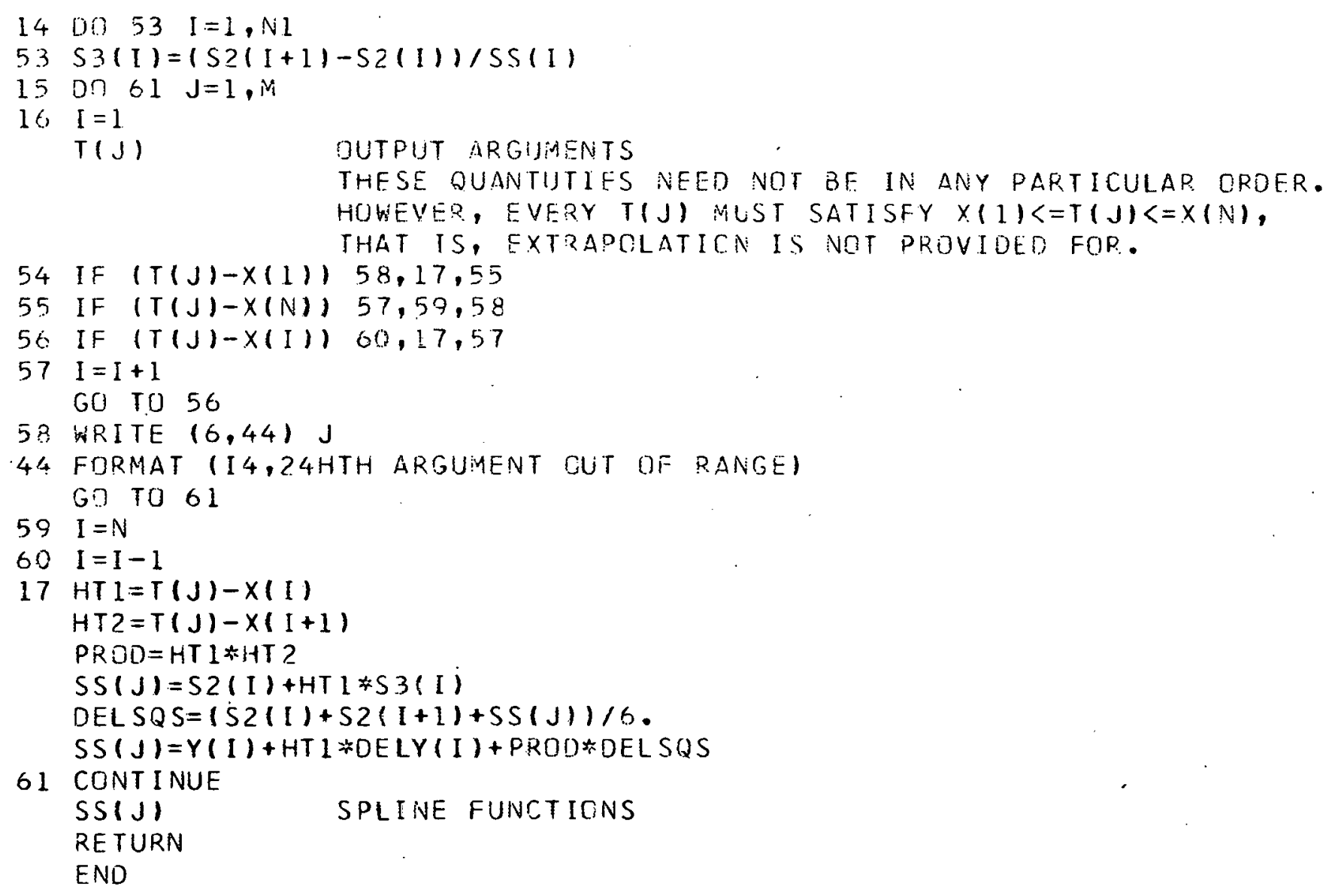


INPUT DATA OF CF-252 SPECTRA

$\begin{array}{lllllll}91 & 215 & 2 & 200 & 4 & 0.42470 & 0\end{array}$

$\begin{array}{lllllllll}002236 & 002261 & 002357 & 002228 & 002112 & 002133 & 002077 & 002011 & 001599\end{array}$ $002046 \quad 001990 \quad 001927 \quad 001928 \quad 001755 \quad 001785 \quad 001896001875001607$ $001716 \quad 001718 \quad 001744 \quad 001722 \quad 001725 \quad 001501001637001514001615$ $001568 \quad 0016 C 8 \quad 001682 \quad 001520 \quad 001491 \quad 001499001550001539001623$ $\begin{array}{llllllllll}001423 & 001526 & 001483 & 001482 & 001495 & 0 & 014 \overline{2} & 001384 & 001431 & 001631\end{array}$ $\begin{array}{llllllllll}001317 & 001341 & 001318 & 001365 & 001290 & 001259 & 001303 & 001308 & 001639\end{array}$ $001239001274 \quad 001294 \quad 001208001194001262 \quad 001237001241001647$ $\begin{array}{llllllllll}001187 & 001288 & 001261 & 001181 & 001138 & 001170 & 001184 & 001196 & 001655\end{array}$ $001137: 001179001143001130001144001120001121001125001663$ $001174 \quad 001102 \quad 001172001143.001129001126 \quad 001159001185001671$ 001064001143001107001059001100001048001066001158001679 $001086001079 \cdot 001133001031001051 \quad 001082 \quad 001054 \quad 001028001687$ 001013001046001034001071001014001065001018001034001695 $001027 \quad 001075001047001088 \quad 000989001026 \quad 001085001057001703$ $001050 \quad 001049001068001032001013000999001109001004001711$ 001009001027001079001047000974000939001025001023001719 $001022001097 \quad 001018000999001011001010 \quad 001014000949001727$ 000967000964001010000943000967000983000993000990001735 $000993 \quad 000938001008 \quad 000984000992000967001025000980001743$ $00097400099300104300095400096600099 \% 000910001033001751$ $000969000936000990 \quad 000981000968000955001021001069001759$ $000960 \quad 000994000952000962000971000973 \quad 000938000976001767$ $000962000990 \quad 001013001045001011000957000982001028001775$ $001020 \quad 001041000914.000934000956 \quad 000924000946000931001783$

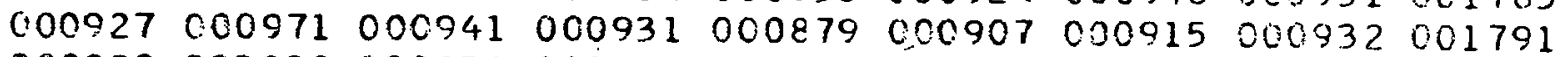
$000890 \quad 000928000959000930000881000919000955000907001799$

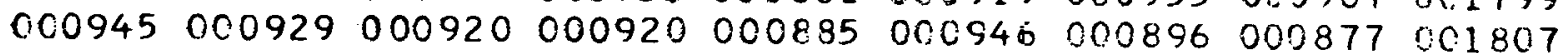


INPUT DATA OF BLANKET SPECTRA

$\begin{array}{llllllllll}106 & 100 & 7 & 100 & 2 & 0.90200 & 1 & & \\ 003920 & 004106 & 004033 & 004281 & 004098 & 004545 & 004728 & 005073 & 000719 \\ 004935 & 005060 & 004907 & 004742 & 004598 & 004600 & 004660 & 004580 & 000727 \\ 004389 & 004531 & 004366 & 004474 & 004224 & 004343 & 004327 & 004306 & 000735 \\ 004183 & 004334 & 004284 & 004064 & 003932 & 004063 & 0041.12 & 003918 & 000743 \\ 003895 & 004028 & 004022 & 003734 & 003797 & 003851 & 003867 & 003955 & 000751 \\ 003806 & 003824 & 003886 & 003899 & 003636 & 003730 & 003791 & 03320 & 00759 \\ 003627 & 003799 & 003852 & 003724 & 003653 & 003737 & 003741 & 003751 & 000767 \\ 003658 & 003784 & 003769 & 003595 & 003528 & 003600 & 003604 & 003673 & 000775 \\ 003592 & 003660 & 003679 & 003643 & 003465 & 03675 & 003538 & 003770 & 000783 \\ 003681 & 003572 & 003675 & 003513 & 003597 & 003600 & 003548 & 003693 & 000791 \\ 003618 & 003768 & 003709 & 003590 & 003544 & 003523 & 003533 & 003720 & 000799 \\ 003725 & 003636 & 003731 & 003469 & 003550 & 003630 & 003591 & 003637 & 000807 \\ 003538 & 003642 & 003510 & 003528 & 003409 & 003606 & 003425 & 003472 & 000815\end{array}$


THE ANALYSIS OF STANDARD NEUTRON SOURCE CF-25?

\section{PARAMETERS AND INPUT DATA FOR RUN 91215 CHANNELS}

LOW CHANNEL NUMBER WHICH BEGINS ANALYSIS 2

ENERGY PER CHANNEL NUMBER (KEV) 0.42470

DIMENSION OF MATRIX 50

THE ENERGY ARRAY OF NEUTRON IN KEV

$\begin{array}{lllllllllllllll}0.77452 E & 03 & 0.85070 E & 03 & 0.93155 E & 03 & C .10139 E & 04 & 0.10964 E & 04 & 0.11736 E & 04\end{array}$ $\begin{array}{llllllllllllll}0.12604 E & 04 & 0.13415 E & 04 & 0.14219 E & 04 & 0.15017 E & 04 & 0.15808 E & 04 & 0.16593 E & 04\end{array}$ $\begin{array}{lllllllllllll}0.17372 E & 04 & 0.18145 E & 04 & 0.18913 E & 04 & 0.19675 E & 04 & 0.20433 E & 04 & 0.21185 E & 04\end{array}$ $\begin{array}{llllllllllllll}0.21933 E & 04 & 0.22676 E & 04 & 0.23415 E & 04 & 0.24150 E & 04 & 0.24881 E & 04 & 0.25609 E & 04\end{array}$ $\begin{array}{llllllllllllll}0.26332 E & 04 & 0.27053 E & 04 & 0.27769 E & 04 & 0.28483 E & 04 & 0.29194 E & 04 & 0.29901 E & 04\end{array}$

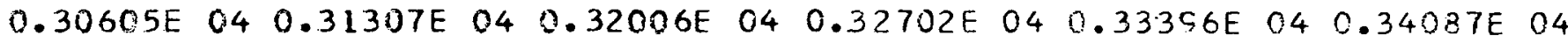
$\begin{array}{lllllllllllllll}0.34776 \mathrm{E} & 04 & 0.35462 \mathrm{E} & 04 & 0.36146 \mathrm{E} & 04 & 0.36828 \mathrm{E} & 04 & 0.37507 \mathrm{~F} & 04 & 0.38185 \mathrm{E} & 04\end{array}$

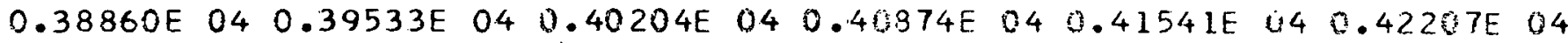
$0.42870 E \quad 04 \quad 0.43532 E \quad 04$

THE MEASURED NEUTRON FLUX CF CF-252

$\begin{array}{llllllllllllll}0.43678 E & 02 & 0.36996 E & 02 & 0.31381 E & 02 & 0.26994 E & 02 & 0.23743 E & 02 & 0.21285 E & 02\end{array}$

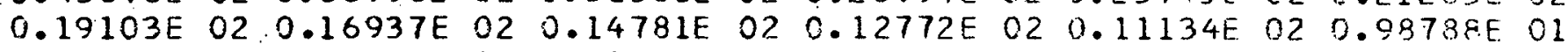

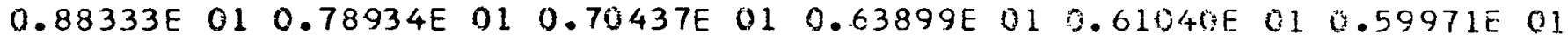
$\begin{array}{llllllllllllll}0.55959 E & 01 & 0.48554 E & 01 & 0.41208 E & 01 & 0.35967 E & 01 & 0.33232 E & 01 & 0.32544 E & 01\end{array}$ $\begin{array}{llllllllllllll}0.32764 E & 01 & 0.32312 E & 01 & 0.29545 E & 01 & 0.25623 E & 01 & 0.23864 E & 0.21 & 0.23944 E & 01\end{array}$

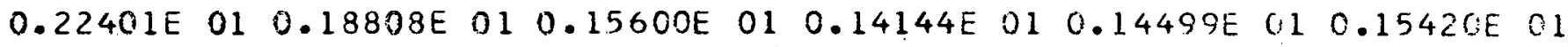

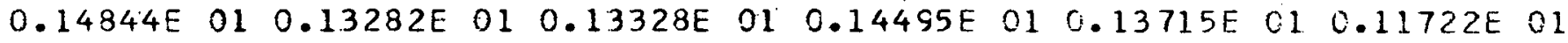
$0.12417 \mathrm{~F} \quad 010.1481 \mathrm{EE} \quad 010.13757 \mathrm{E} \quad 010.71384 \mathrm{E} \quad 00 \quad 0.55184 \mathrm{~F} \quad 00 \quad 0.44518 \mathrm{E} \quad 00$ $0.4454 C E$ OO $0.46150 E$ OO 


\section{OUTPUT DATA}

THE CALCULATED NEUTRON FLUX OF CF-252

$\begin{array}{llllllllllll}0.15941 E & 02 & 0.15816 E & 02 & 0.15615 E & 02 & 0.15354 E & 02 & 0.15046 E & 02 & 0.14704 E & 02\end{array}$

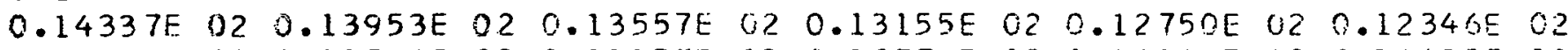

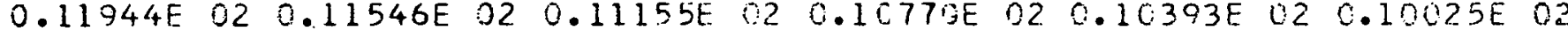

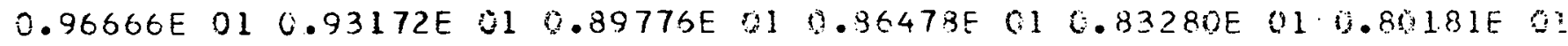

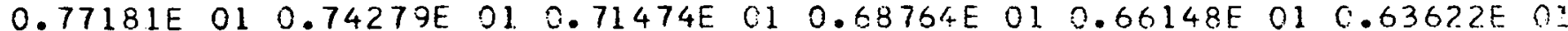

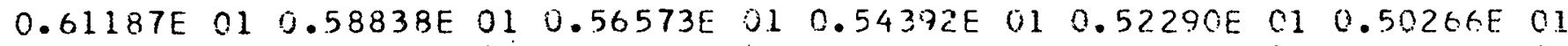

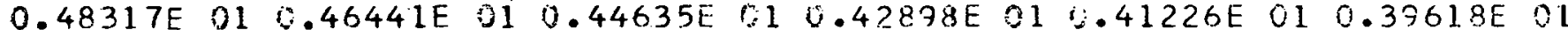
$\begin{array}{llllllllllllllll}0.38072 E & 01 & 0.36585 E & 01 & 0.35154 E & 01 & 0.33780 E & 01 & 0.32458 E & 0 & 0.31187 E & 01\end{array}$ $0.29966 E \quad 010.28792 E \quad 01$

THE CORRECTION DIAGONAL MATRIX FOR THE SYSTEM

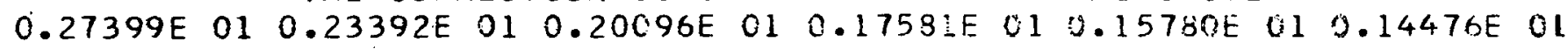

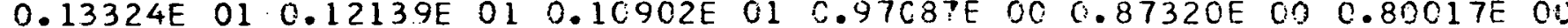
$0.73957 E$ OO $0.68363 E$ DO $0.63146 E$ OO 0.5933 OE OC $0.58729 E$ OC $0.59819 E$ OU

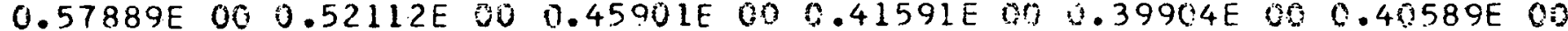
$\begin{array}{llllllllllllll}0.42451 E & O O & 0.43501 E & 00 & 0.41337 E & 00 & 0.37262 E & 00 & 0.36077 E & 00 & 0.37635 E & 0.3\end{array}$

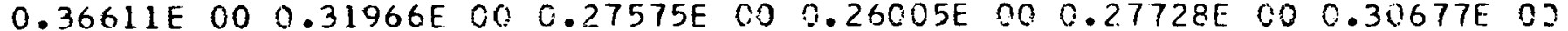
$0.30722 E$ CO $0.28599 E$ DO $0.29859 E$ DO $0.33789 E$ OO $0.33269 E$ OO $0.29587 E$ OJ

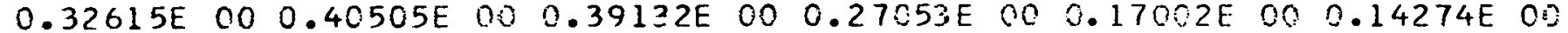
$0.14863 E \quad 00 \quad 0.16029 E$ DO 
THE ANALYSIS OF UNKNOWN MEUTRON ENERGY SPFCTPA

PARAMETERS AND INPUT DATA FOR RUN $106 \quad 100$ CHANNELS

LOW CHANNEL NUMBER WHICH BEGINS ANALYSIS 7 ENERGY PER CHANNEL NUMBEF: IKEVI0.90200

DIMENSION OF MATRIX 44

THE ENERGY ARRAY OF NEUTRON IN KEV

$\begin{array}{llllllllllllllll}0.78122 E & 03 & 0.86303 E & 03 & 0.94932 E & 03 & 0.10369 E & 0.4 & 0.11244 E & 04 & 0.12116 E & 04\end{array}$ $\begin{array}{llllllllllllll}0.12981 \mathrm{E} & 04 & 0.13839 \mathrm{E} & 04 & 0.14689 \mathrm{E} & 04 & 0.15532 \mathrm{E} & 04 & 0.16368 \mathrm{E} & 04 & 0.17197 \mathrm{E} & 04\end{array}$ $\begin{array}{lllllllllllll}0.18019 E & 04 & 0.18835 E & 04 & 0.19645 E & 04 & 0.20449 E & 04 & 0.21248 E & 04 & 0.22041 E & 04\end{array}$ $\begin{array}{lllllllllllllll}0.22830 E & 04 & 0.23614 E & 04 & 0.24393 E & 04 & 0.25168 E & 04 & 0.25939 E & 04 & 0.26705 E & 04\end{array}$ $\begin{array}{lllllllllllllll}0.27468 E & 04 & 0.28227 E & 04 & 0.28983 E & 04 & 0.25735 E & 04 & 0.3 C 484 E & 04 & C .31229 E & C 4\end{array}$ $\begin{array}{llllllllllllll}0.31972 \mathrm{~F} & 04 & 0.32711 \mathrm{E} & 04 & 0.33448 \mathrm{~F} & 04 & 0.34181 \mathrm{E} & 04 & 0.34912 \mathrm{E} & 04 & 0.3564 & 0.3 \mathrm{E} & 04\end{array}$ $\begin{array}{llllllllllll}0.36366 E & 04 & 0.37089 E & 04 & 0.3781 O E & 04 & 0.38528 E & 04 & 0.39244 E & 04 & 0.39958 E & 0.4\end{array}$ $0.40669 \mathrm{E} \quad 040.41378 \mathrm{E} \quad 04$

THE CORRECTED DIAGONAL RESPCNSE MATRIX

$\begin{array}{llllllllllllll}0.71213 E & 02 & 0.62625 E & 02 & 0.55688 E & 02 & 0.50539 E & 02 & 0.47178 E & 02 & 0.44786 E & 02\end{array}$ $\begin{array}{lllllllllllll}0.42318 \mathrm{E} & 02 & 0.39365 \mathrm{E} & 02 & \mathrm{U} & 0.36072 \mathrm{E} & 02 & 0.33114 \mathrm{E} & 02 & 0.31002 \mathrm{E} & 02 & 0.29420 \mathrm{E} & 02\end{array}$ $0.27960 E$ O2 $0.26526 E$ O2 $0.25571 E$ O2 $0.26 C 99 E$ 02 $0.27431 E$ C2 $0.27079 E \quad 02$ $\begin{array}{llllllllllllll}0.24780 E & 02 & 0.22419 E & 02 & 0.21170 E & 02 & 0.21409 E & 02 & 0.22886 E & 02 & 0.2468 .7 E & 02\end{array}$

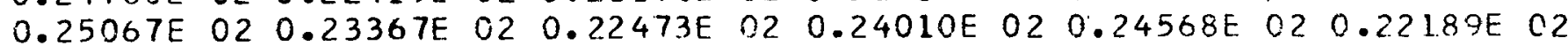

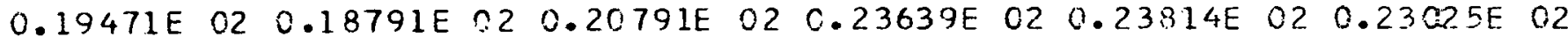

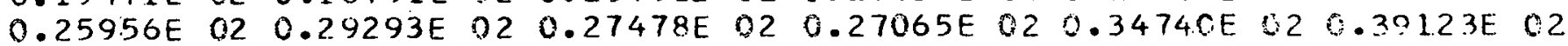
$0.30299 E \quad 02 \quad 0.18782 E \quad 02$ 
OUTPUT DATA

THE NEUTRON ENERGY SPECTPA FRDM BLANKET

$0.20349 E \quad 02 \quad 0.20619 E \quad 02 \quad 0.20854 E$ V.2 $0.21007 E$ O2 $0.20790 E$ C. $0.20509 E \quad 02$

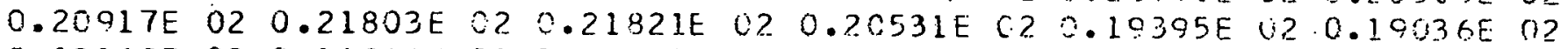

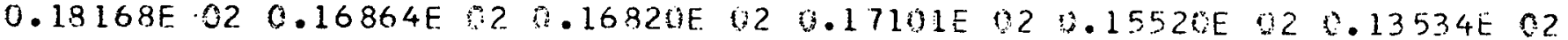
$0.13538 E$ O2 $0.15280 F \quad 020.15983 E$ O2 $0.14780 E 020.13729 E$ C? $0.12959 E$ O?

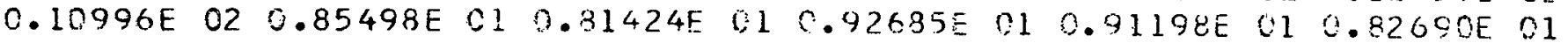

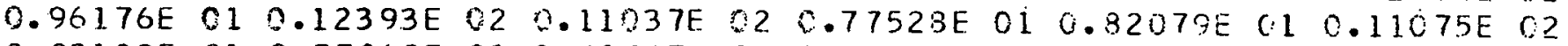

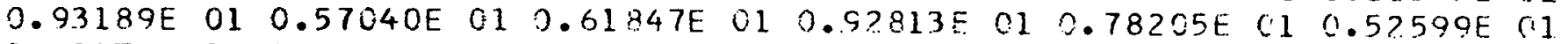
$0.49173 \mathrm{E}$ OL $0.74507 \mathrm{E} 01$ 
250

Appendix G

COMPUTER PROGRAM COMPS

The computer code COMPT performs the analys is of the Compton continuum gamma-ray spectrum from Blanket No. 2 . The integration is done by an iterative unfolding method. Input data are the smoothed background output data from GAMANL. Output data consist of the upper triangular response matrix as well as the photon flux spectrum from Blanket No. 2 


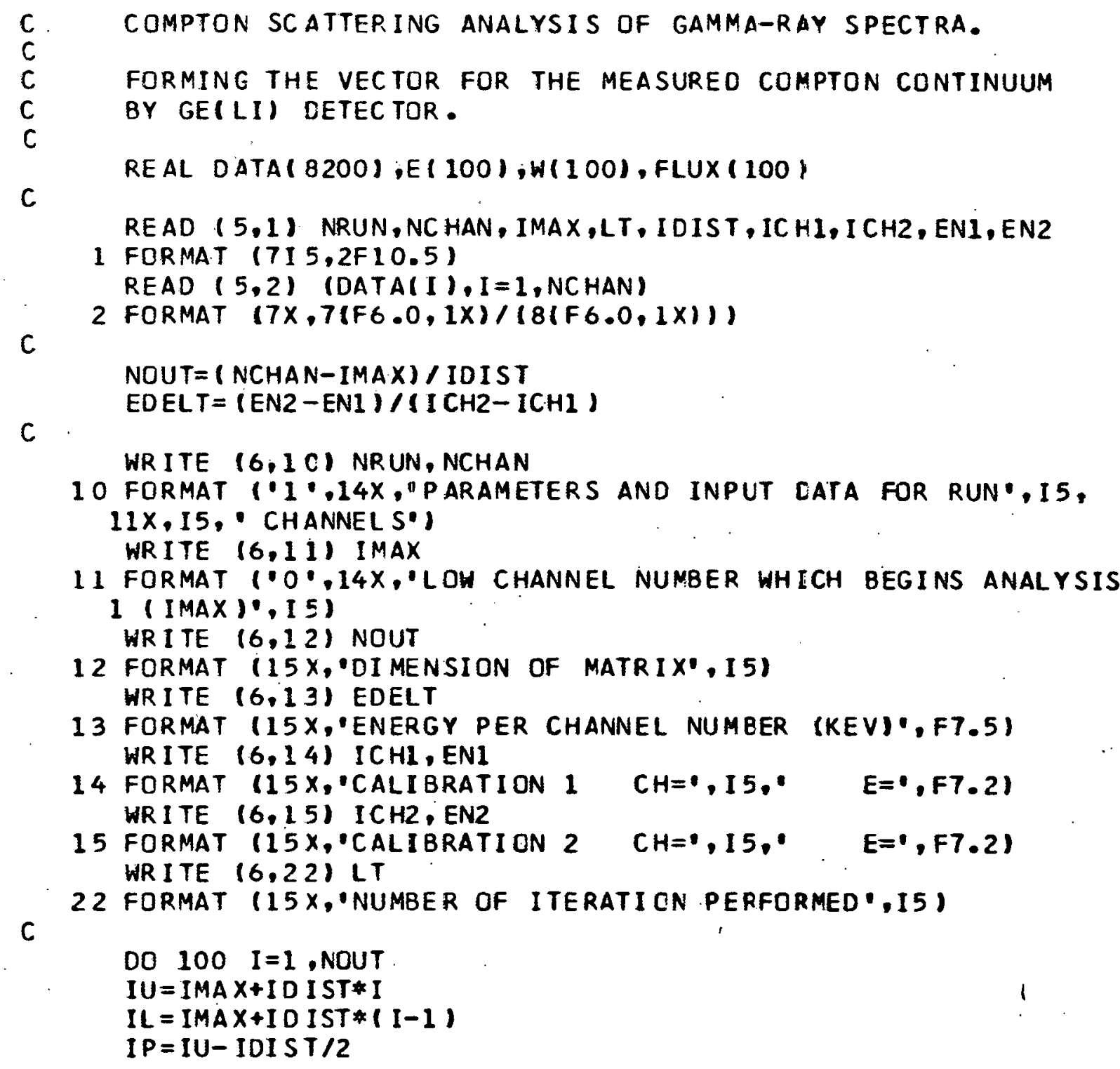


$E(I)=(E N 1+(I P-I C H I) * E D E L T) / 511$. $W T=D A T A(I L)$

$\mathrm{IL}=\mathrm{IL}+\mathrm{I}$

$I I=2$

IK $=2$

DO $101 \mathrm{~J}=\mathrm{IL}, I U$

$\mathrm{L}=1$

DO $102 \mathrm{~K}=1$, IK

$102 \mathrm{~L}=(-1) * \mathrm{~L}$

$\therefore I 1=I 1+2 * L$

$I K=I K+1$

IF (J.EQ.IU) $I 1=1$

$W T=W T+D A T A(J) * I 1$

101 CONTINUE

$W(I)=W T /(3$ *IDIST)

C

100 CONTINUE

WR I TE $(6,16)$

16 FORMAT $(\cdot 0 \cdot, 14 X, \cdot$ THE MEASURED COMPTCN CONTINUUM VECTOR $)$

WRITE $(6,17)(W(I), I=1$, NOUT)

$\mathrm{c}$

17 FORMAT (GE 12.5)

C FORMING THE RESPONSE MATRIX

C IT $=1$

DO. $103 \quad I=1$, NOUT

DO $104 \mathrm{~J}=\mathrm{I}$, NOUT

$I U=I M A X+I D I S T * J$

$E U=(E N I+(I U-I C H I) * E D E L T) / 511$.

$E U=E U *(1 \bullet+S Q R T(1 \bullet+2 \cdot / E U)) / 2$.

$I L=I M A X+I D I S T *(J-1)$

$E L=(E N 1+(I L-I C H L) * E D E L T) / 511$

$E L=E L * 11 .+\operatorname{SORT}(1 .+2 . / E L)) / 2$.

$E D I S T=(E U-E L) / I D I S T$

$N I=I D I S T+1$

$C T=E L-E(I)$ 
$C T=E(I) * E(I) /(E L * E L * E L * E L * C T * C T)+2 . /(E L * E L)$

$1+((E(I)-1) *(E(I)-1)-1) /(E L * E L * E L * C T)$

$11=2$

DO $105 \mathrm{~K}=2$, NI

$E M=E L+E D I S T *(K-1)$

$L=1$

DO $106 \mathrm{Kl}=1, \mathrm{~K}$

$106 L=(-1) * L$

$I I=I 1+2 * L$

IF (K.EQ.N I) II $=1$

$C=E M-E(I)$

$C=E(I) * E(I) /(E M * E M * E M * E M * C * C)+2 . /(E M * E M)$

$1+((E(I)-1) *(E(I)-1)-1) /(E M * E M * E M * C)$

$\mathrm{CT}=\mathrm{CT}+\mathrm{C}$

105 CONTINUE

DATA $(I T)=C T \# E D I S T / 3$.

$I T=I T+1$

104 CONTINUE

$E(I)=511 . * E(I) *(1 .+\operatorname{SQR} T(1+2 . / E(I))) / 2$

103 CONTINUE

WR ITE $(6,18)$

18 FORMAT $(1,14 \times$, 'THE ENERGY ARRAY OF PHOTON IN KEV')

WRITE $(6,17)$ (E(I), I=1, NOUT)

WRITE $(6,19)$

1.9 FORMAT $1.0 \% 14 \times$, "THE UPPER TRI ANGULAR RESPONSE MATRIX')

$J 1=1$

$\mathrm{J} 2=\mathrm{NOU} T$

DO $107 \mathrm{I}=1$, NOUT

WR ITE $(6,20)$ I

20 FORMAT $(15 \mathrm{X}, 11=1,15)$

WRITE $(6,17)$ (DATA (J), J $=\mathrm{J} 1, \mathrm{~J} 2$ )

$\mathrm{J1}=\mathrm{J} 2+1$

$J 2=J 1+($ NOU T $T-I)-1$

$F(U X(I)=1$.

107 CONTINUE 


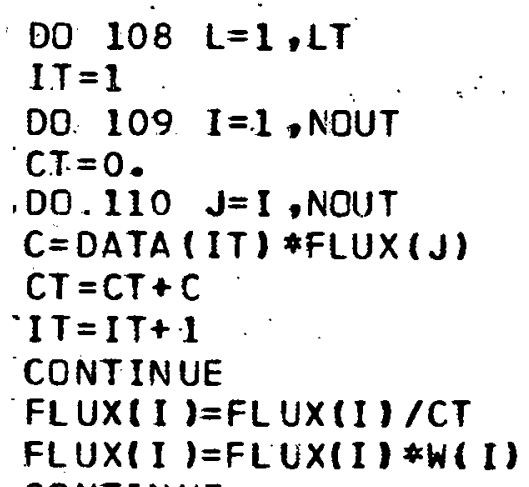


INPUT DATA

$\begin{array}{cccccccc}1054095 & 81 & 128 & 50 & 184 & 522 & 511.000001293 .60000 \\ & 0 . & 0 . & 0 . & 0 . & 0 . & 0 . & 0 . \\ 0 . & 0 . & 0 . & 0 . & 0 . & 0 . & 0 . & 0 . \\ 0 . & 0 . & 0 . & 0 . & 0 . & 0 . & 0 . & 0 . \\ 0 . & 0 . & 0 . & 0 . & 0 . & 0 . & 0 . & 0 . \\ 0 . & 0 . & 0 . & 0 . & 0 . & 0 . & 0 . & 0 . \\ 0 . & 0 . & 0 . & 0 . & 0 . & 0 . & 0 . & 0 . \\ 0 . & 0 . & 0 . & 0 . & 0 . & 0 . & 0 . & 0 . \\ 0 . & 0 . & 0 . & 0 . & 0 . & 0 . & 0 . & 0 . \\ 0 . & 0 . & 0 . & 0 . & 0 . & 0 . & 0 . & 0 . \\ 0 . & 0 . & 0 . & 0 . & 0 . & 0 . & 0 . & 0 .\end{array}$

0. 93902 . 91291.88386 • 85751. 83845 • 82715.81988. 81143.79830.78034. 76045. 7427t. 73039.72363. 71963. 71408. 70405.68998. 67506. 66260.65361.64667.63978. 63220.62462. $61801.61255 .6077 \mathrm{C} .60274$. 59710. 59006. 58069.56851.55431.54035.52916.52194.51780. 51436. 50939. 50206. 49321. 48461.4778 . 47374. 47166.47022. 46773.46318.45677. 44989. 44423.44082. 43940. 43874. 43729.43399.42875. 42263.41740.41583.41293.41003. 40713. 40423 . 40133 . 39842. 39552. 39262 • 38972. 38791. 38610 . 38429 • 38248 . 38067. 37886. 37705. 37523. 37342. 37161 . 36980. 36799 . 36618. 36437. 36256. 36107. 35958. 35808. 35659. 35510. 35360. 35211. 35061. 34912. 34437. 33962. 33486, 33011. 32536. 32171. 31806.31441. 31076. .30711 .30345 . 29980. 29615. 29250.28885 . 28882. 28879. 28876. 28872 . 28869 . 28866. 28863. 28860.28857. 28854.

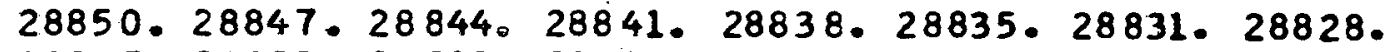
28825. 28822 . $28819 \cdot 28816$. 28813.28809 • 28806. 28803. 28717. 28631 . 28544 . 28458. 28372. 28285. 28199 . 28113. 28027. 27940 • 27854 . 27768. 27682. 27595. 27509. 27423. 27336. 27250.27164. 27078. 26991. 26905• 26819. 26698. 26578. 26457. 26337. 26217. 26096. 25976. 25878. 25780. 25683. 25585 • 25487. 25389.25292.25253.25214. 25175. 
$25137.25098 \cdot 25059 \cdot 24989 \cdot 24918 \cdot 24848 \cdot 24778$.24708. 24637. 24567. 24497. 24427. 24356.24286.24216. 24146. 24075 . 24005 . 23935. 23865. 23794. 23724. 23654. 23584 . 23513.23443. 23373. 23303. 23232. 23150 • 23067. 22984. $22901 \cdot 22818 \cdot 22735.22652$. 22569. 22486. 22437. 22387. 22337. 22287. 22237. 22187. 22137. 22087. 22057. 22026. 21995. 21964.-21934. 21903. 21872. 21802. 21731. 21660. 21589. $21519 \cdot 21448$ • 21377. 21306. 21252 . 21198 . 21144. 21090. 21036. 20982. 20928. 20874. 20819. 20765. 20711 . 20657. 20603. 20549. 20495. 20441. 20387. 20376. 20366. 20356. 20346.20336. 20326. 20316.20305. 20296. 20286. 20276. 20266. 20256. 20247. 20237. 20229. 20221. 20214. 20206. 20198 • 20190. 20183. 20175. 20167.20097. 20026. 19955. 19885. 19814. 19743. 19673. 19671. 19669.'19667. $19665.19664 .19662 .19660 .1965 \varepsilon_{0}$. 19647. 19635. 19623. 19612.19600. 19589. 19577. 1956t. 19554. 19522. 19490. 19458.19426.19294. 19362. 19330. 19298. 19279. 19260. 19241.19222 . 19204. 19185. 1916 . . 19080. 18994. 18907. 18821.18735.18648.18562. 18476.18389.18303. 18217. 18130.18044. 17958. 17871. 17785.17699. 17612. 17526. 17440.17353.17267. 17181. 17094.17008. 16922. 16835. 16749.16663 .16576 .16490 .16404 .16317 .16231 .16145$. 16059.15972 . 15886.15800 .15713 .15627 .15542 .15458$. 15374. 15289. 152C5. 15120. 15036. 14952. 14867. 14786. 14704. 14623.14541. 14460.14378. 14297. 14208. 14120 . 14031. 13942. 13853. 13765. 13676.13557. 13584. 13612. 13588. 13515. 13496. 13404. 13312.13220.13128. 13036. 12944. 12852 . 12760. 12668. 12576. 12484. 12446. 12407 . 12369. 12330. 12292. 12253. 12215. 12176. 12138. 12099. 12060.12022 . 11983. 11945. 11906.11868.11829.11801. 11774. 11746. 11718. 11690.11662.11634. 11606. 11578. 11550. 11508 . 11466 . 11424. 11382. 11339. 11297. 11255. 11052. 10850. 10647. 10444. 10242. 10039.9952. 9865. 9778.9691 .9604 .9517 .9430 .9343 .9256 .9169$.

9169. 9169.9169.9168.9168.9168.9168.998.

9168.9168.9168.9168.9168.9168.9168.9167. 


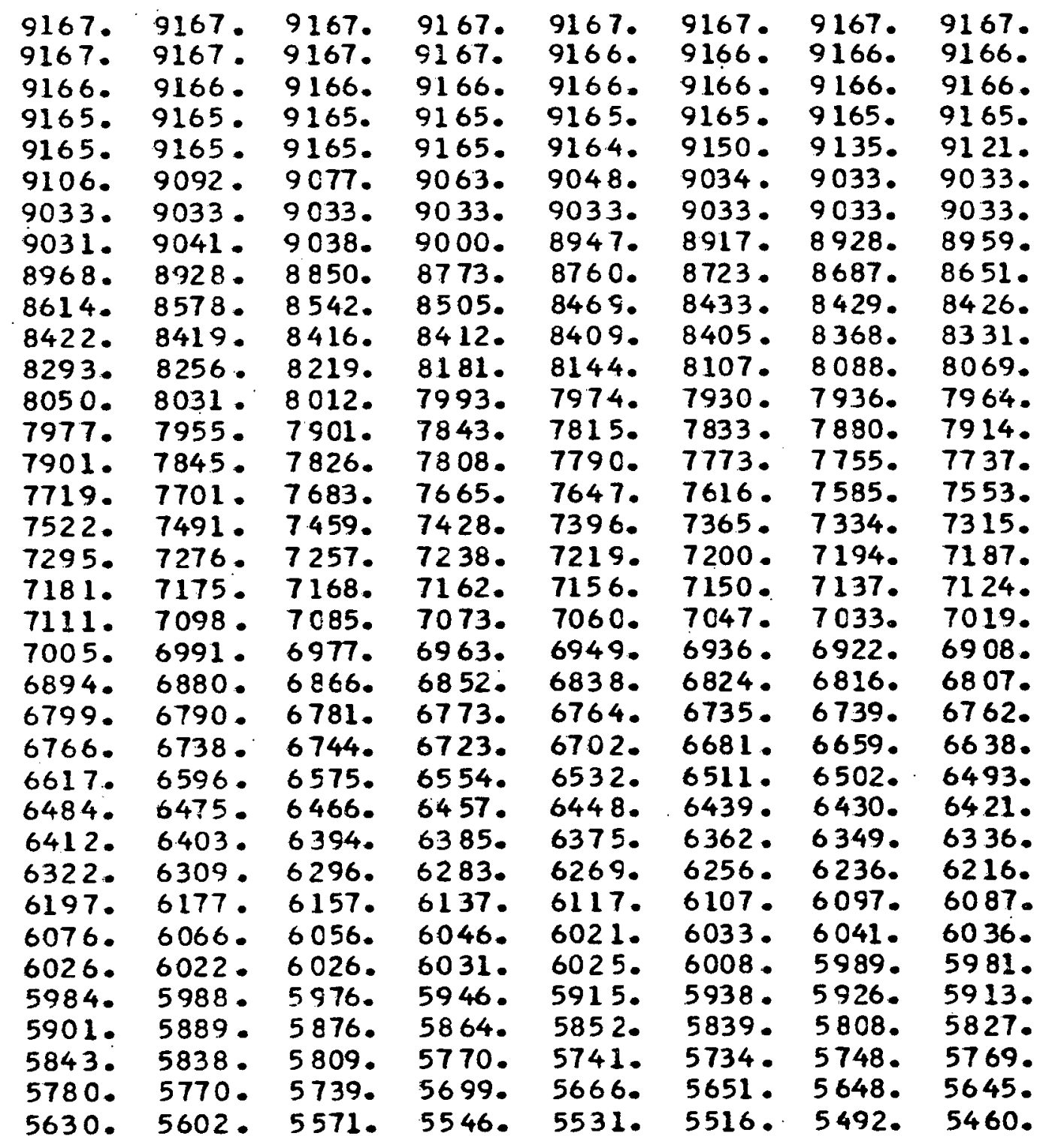




\begin{tabular}{|c|c|c|c|c|c|c|c|}
\hline $\begin{array}{l}5431 . \\
5262 . \\
5126 . \\
5042 . \\
4890^{\circ} \\
4781 . \\
405 . \\
4656 . \\
4577 \\
4995 \\
4421 . \\
4363 . \\
4307 . \\
4232 . \\
4179 . \\
4112 . \\
4068 . \\
4036 .\end{array}$ & 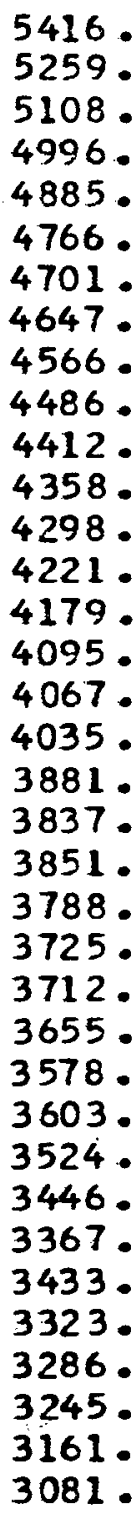 & 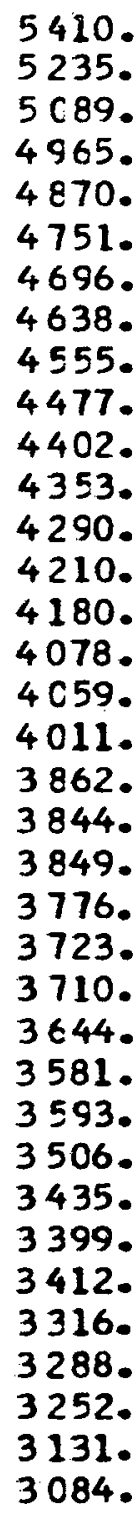 & $\begin{array}{l}519 \\
507 \\
497 \\
485 \\
473 \\
469 \\
462 \\
454 \\
446 \\
439 \\
434 \\
428 \\
419 \\
418 \\
406 \\
404 \\
397 \\
384 \\
385 \\
384 \\
376 \\
372 \\
370 \\
363 \\
357 \\
356 \\
351 \\
342 \\
341 \\
337 \\
330 \\
327 \\
324 \\
308 \\
308\end{array}$ & $\begin{array}{l}514 \\
501 \\
495 \\
484 \\
472 \\
468 \\
461 \\
453 \\
445 \\
438 \\
434 \\
427 \\
418 \\
418 \\
401 \\
402 \\
393 \\
382 \\
385 \\
384 \\
375 \\
372 \\
370 \\
362 \\
355 \\
353 \\
350 \\
341 \\
341 \\
333 \\
330 \\
326 \\
322 \\
305 \\
309\end{array}$ & $\begin{array}{l}0 \\
0 \\
0 \\
0 \\
0 \\
0 \\
0 \\
0 \\
0 \\
0 \\
0\end{array}$ & $\begin{array}{l}3717 \\
3686 \\
3603 \\
3540 \\
3529 \\
3480 \\
3391 \\
3389 \\
3344 \\
3287 \\
3230 \\
3180 \\
3073 \\
3087\end{array}$ & 30 \\
\hline
\end{tabular}




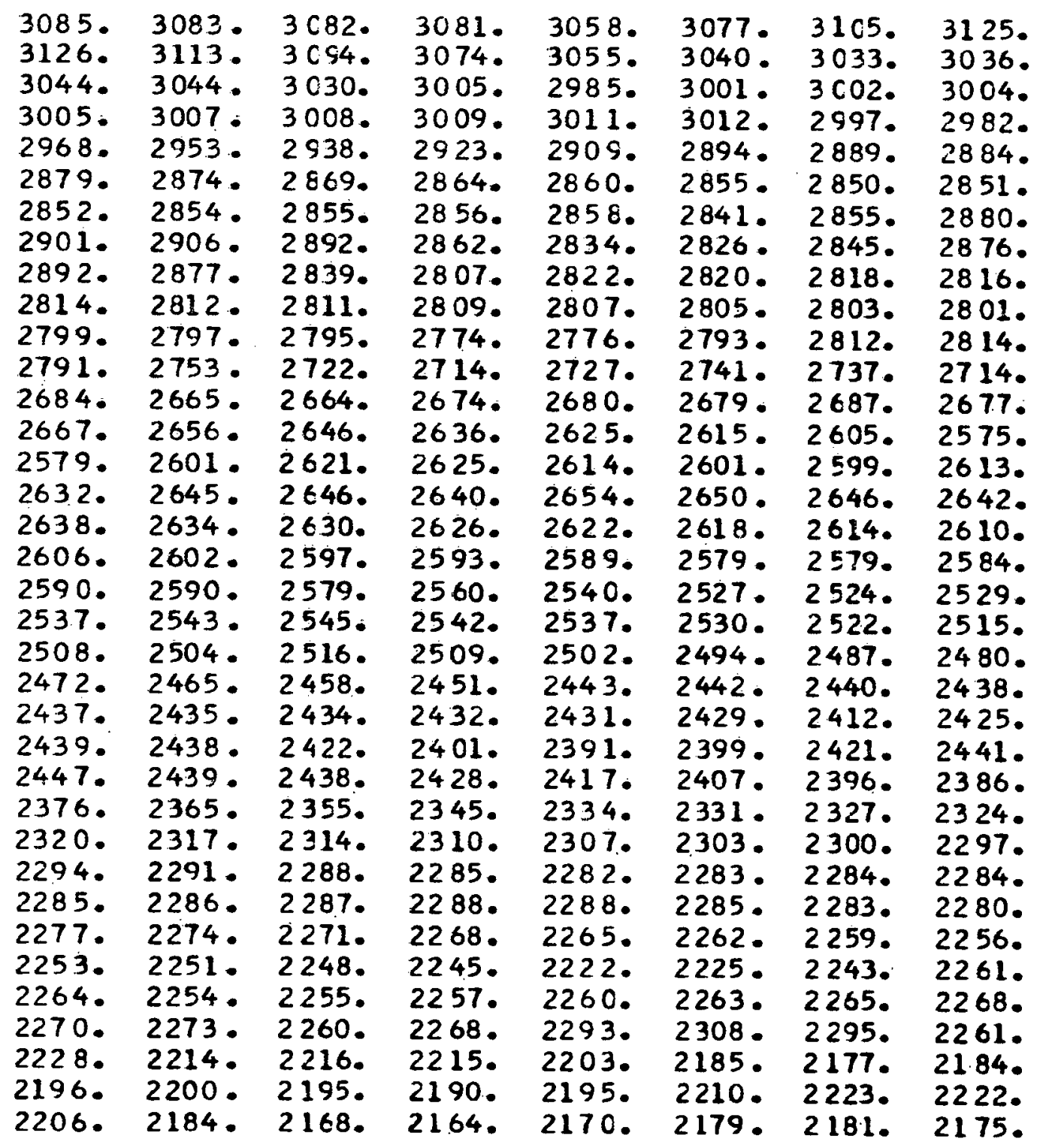




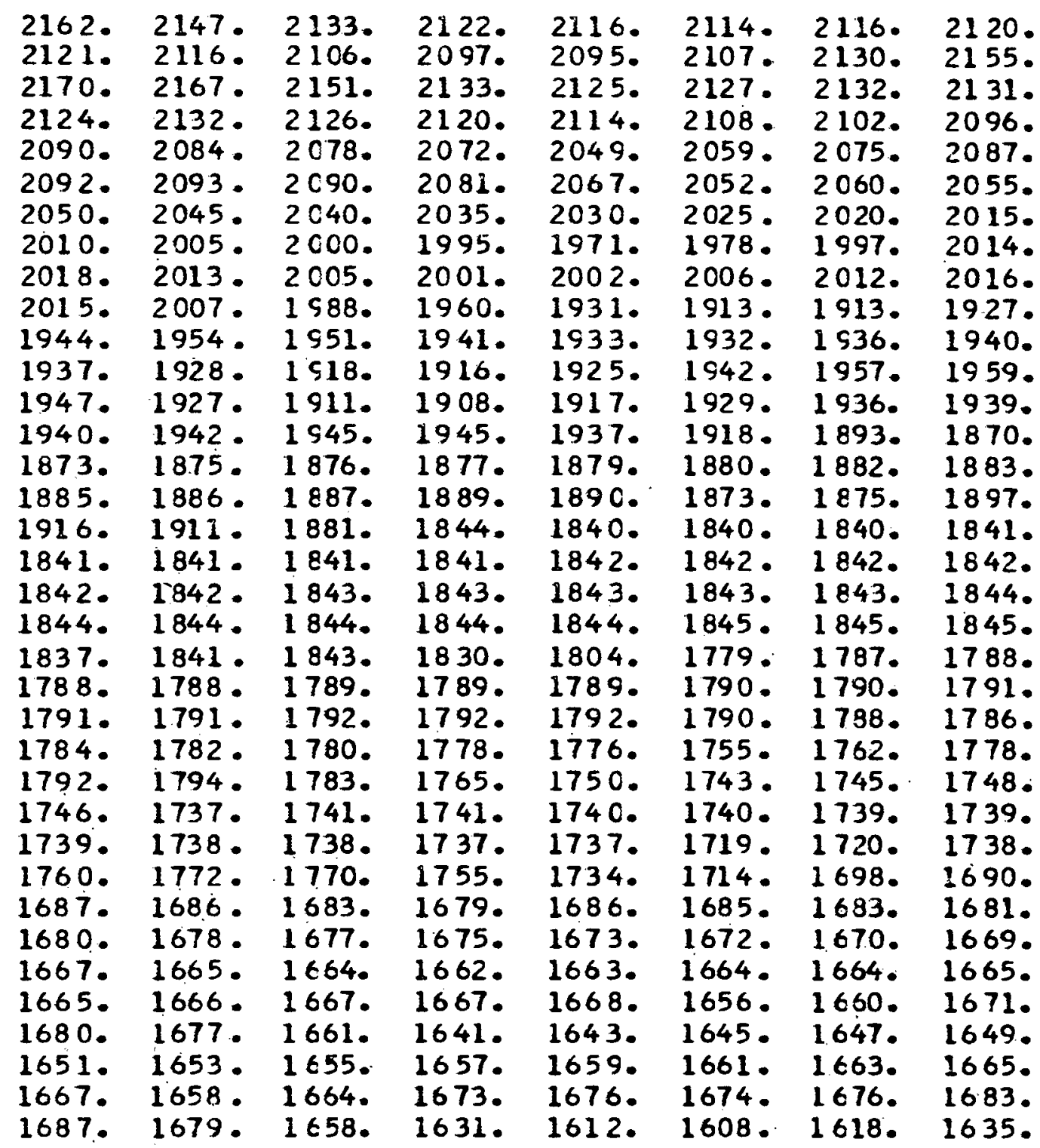




\begin{tabular}{|c|c|c|c|c|c|c|c|}
\hline $\begin{array}{l}586 . \\
602 . \\
555 . \\
556 . \\
523 . \\
491 . \\
483 .\end{array}$ & 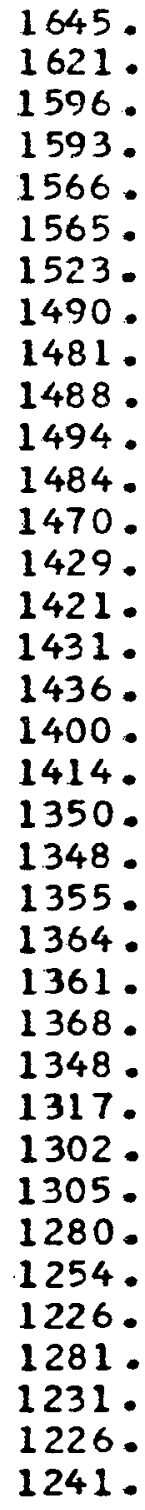 & 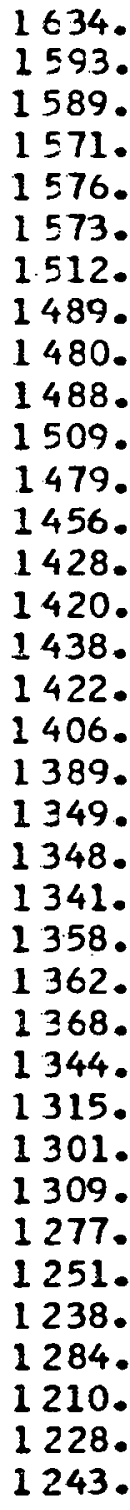 & 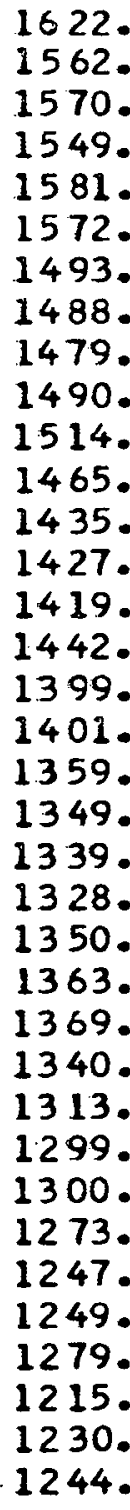 & $\begin{array}{l}1536 \\
1579 \\
1556 \\
1496 \\
1487 \\
1463 \\
149 C \\
1508 \\
1448 \\
1434 \\
1426 \\
1418 \\
1444 \\
1375 \\
1391 \\
1339 \\
1349 \\
1342 \\
1324 \\
1358 \\
1364 \\
1370 \\
1336 \\
1311 \\
1297 \\
1284 \\
127 C \\
1244 \\
1255 \\
1275 \\
1217 \\
1232 \\
1246\end{array}$ & $\begin{array}{l}1530 \\
1532 \\
1571 \\
1534 \\
1495 \\
1486 \\
1469 \\
1485 \\
1494 \\
1438 \\
1433 \\
1425 \\
1407 \\
1442 \\
1358 \\
1388 \\
1350 \\
1349 \\
1348 \\
1331 \\
1358 \\
1364 \\
1365 \\
1331 \\
1310 \\
1295 \\
1293 \\
1267 \\
1241 \\
1254 \\
1276 \\
1219 \\
1234 \\
1241\end{array}$ & 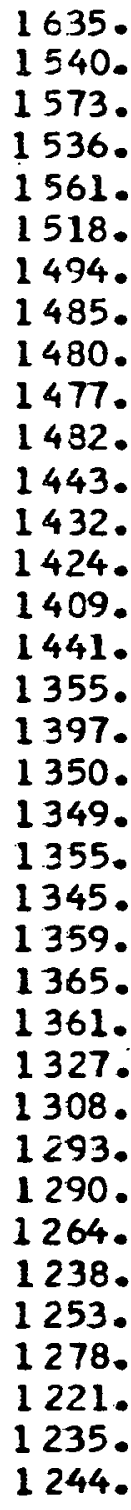 & $\begin{array}{l}12 \\
12 \\
12 \\
12\end{array}$ \\
\hline
\end{tabular}

PAGE 11 


\begin{tabular}{|c|c|c|c|c|c|c|c|}
\hline $\begin{array}{l}260^{\circ} \\
227^{\circ} \\
224{ }^{\circ} \\
242^{\circ} \\
246^{\circ}\end{array}$ & 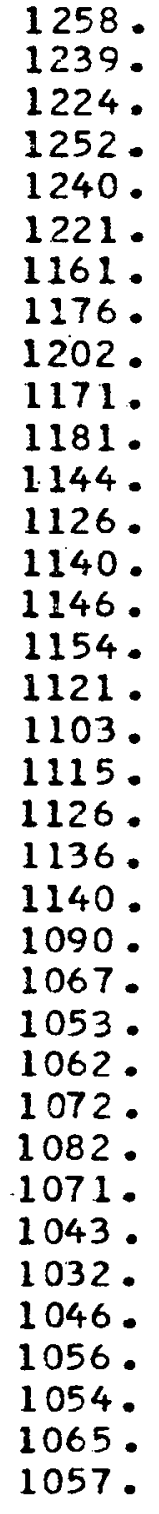 & 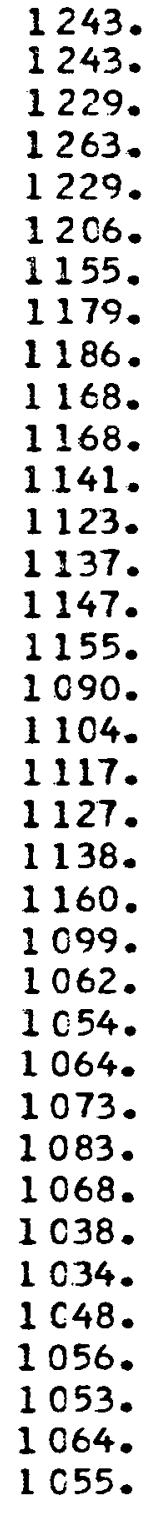 & 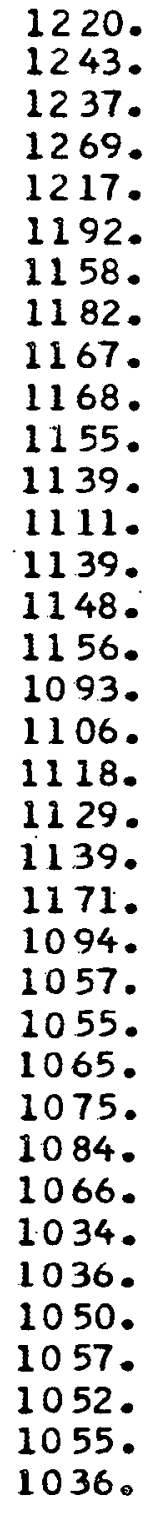 & $\begin{array}{l}11 \\
11 \\
11 \\
11 \\
10 \\
11 \\
11 \\
11 \\
10 \\
11 \\
11 \\
11 \\
11 \\
11 \\
10 \\
10 \\
10 \\
10 \\
10 \\
10 \\
10 \\
10 \\
10 \\
10 \\
10 \\
10 \\
10 \\
10\end{array}$ & 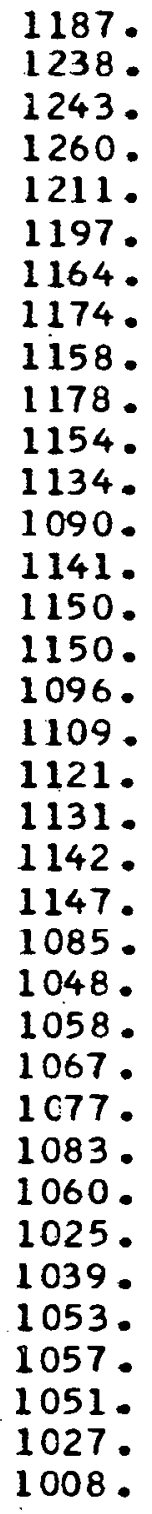 & 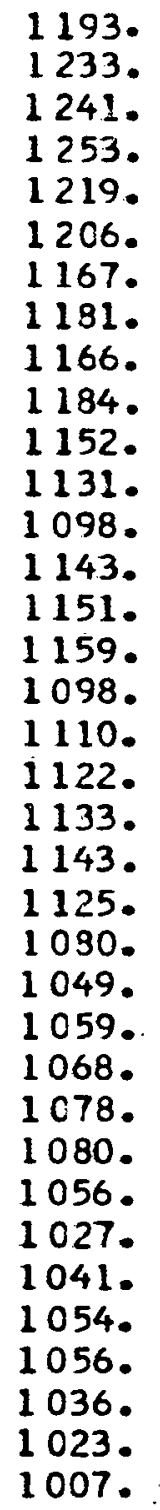 & $\begin{array}{l}11 \\
11 \\
11 \\
11 \\
11 \\
10 \\
10 \\
10 \\
10 \\
10 \\
10 \\
10 \\
10 \\
10 \\
10 \\
10 \\
10 \\
10 \\
10\end{array}$ \\
\hline
\end{tabular}




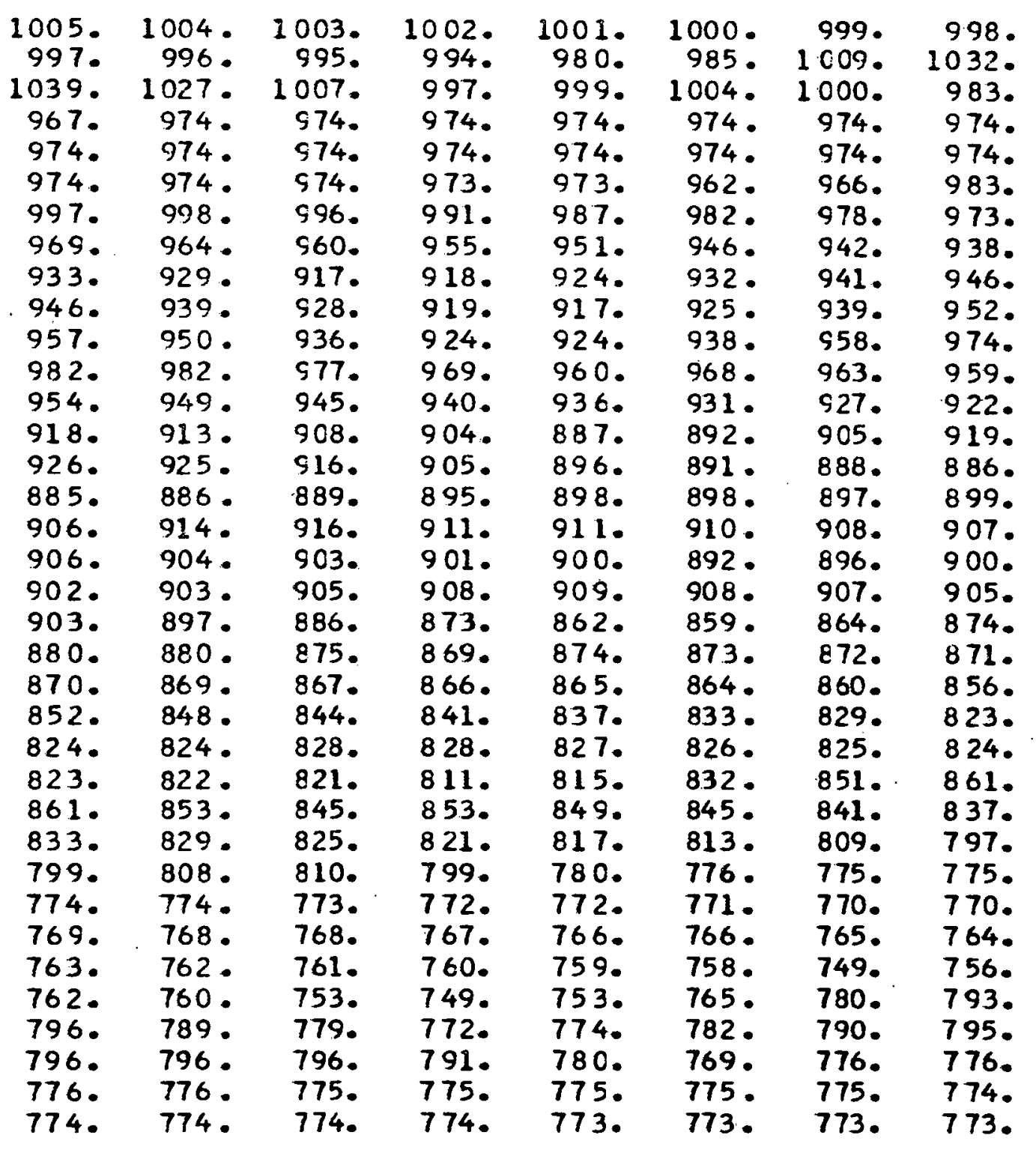




\begin{tabular}{|c|c|c|c|c|c|c|c|}
\hline 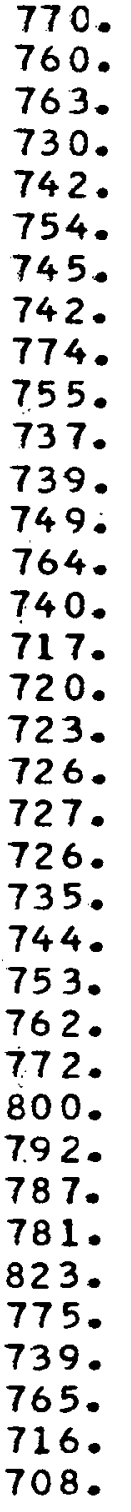 & 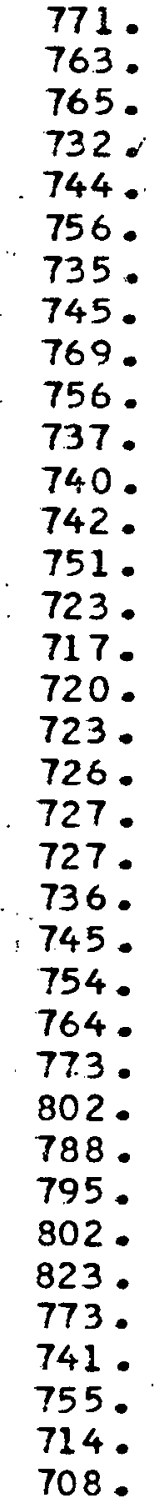 & $\begin{array}{l}769 . \\
759^{\circ} \\
756 . \\
73{ }^{\circ} \\
745 . \\
757 . \\
734 . \\
750^{\circ} \\
765 . \\
754 . \\
738 . \\
740 . \\
737 . \\
733 . \\
708 . \\
717 . \\
720 . \\
723 . \\
726 . \\
727 . \\
728 . \\
737 . \\
746 . \\
755 . \\
765 . \\
774 . \\
796 . \\
777 . \\
805 . \\
818 . \\
809 . \\
772 . \\
745 . \\
743 . \\
713 . \\
709 .\end{array}$ & 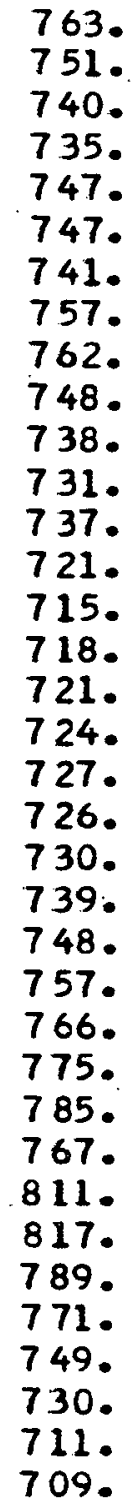 & 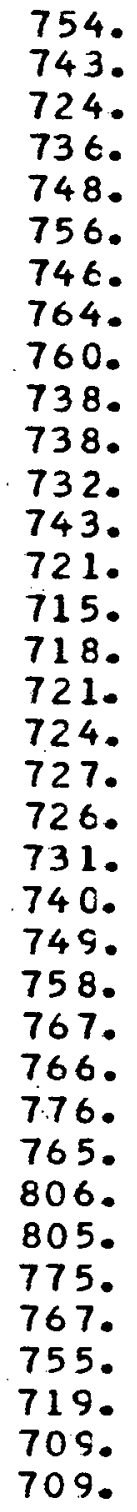 & 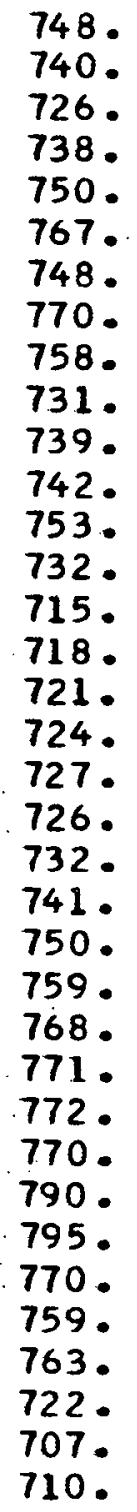 & 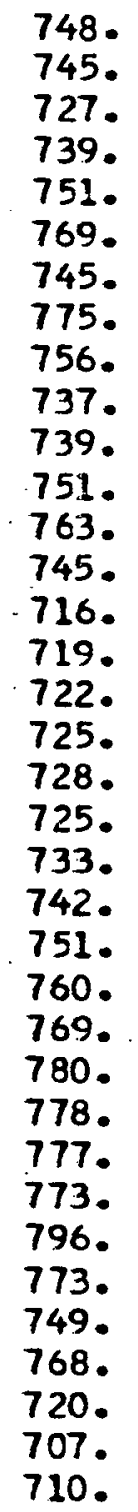 & 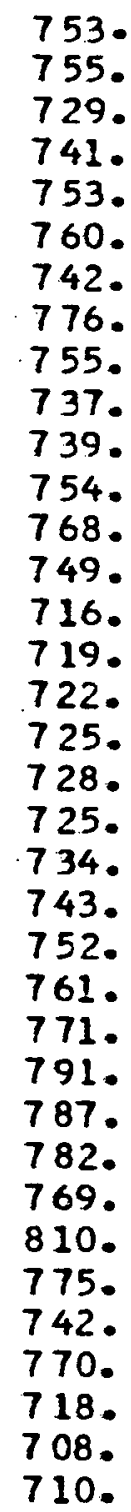 \\
\hline
\end{tabular}

PAGE 14 


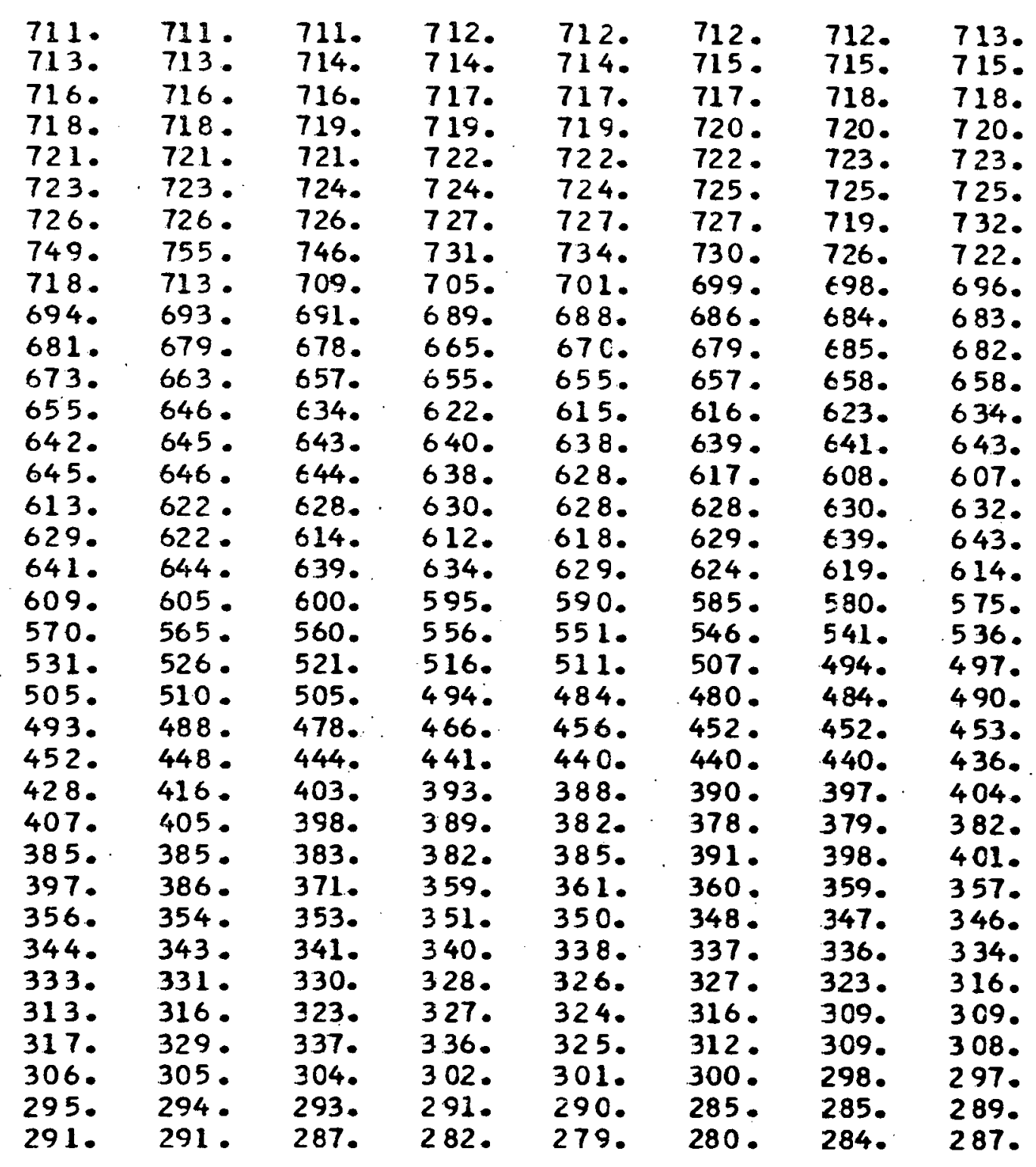

PAGE 15 


\begin{tabular}{|c|c|c|c|c|c|c|c|}
\hline 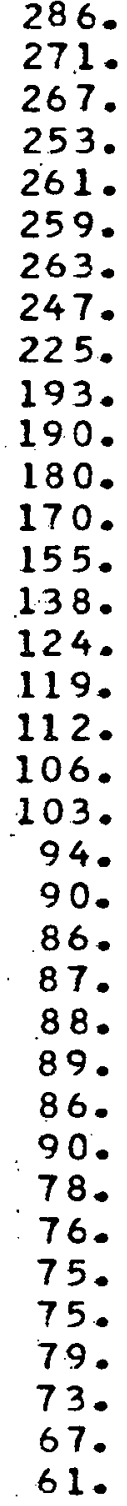 & 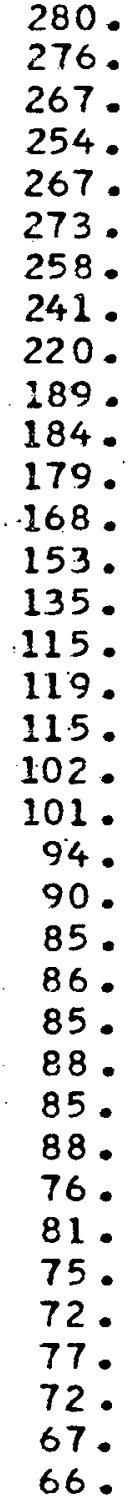 & 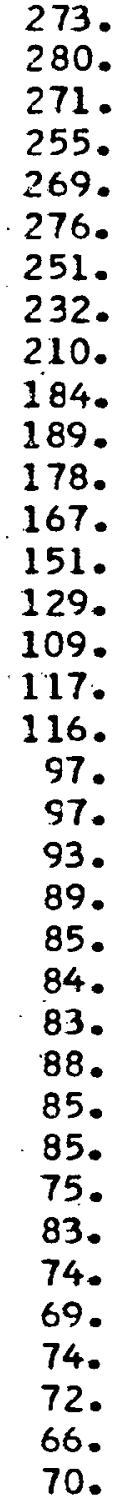 & 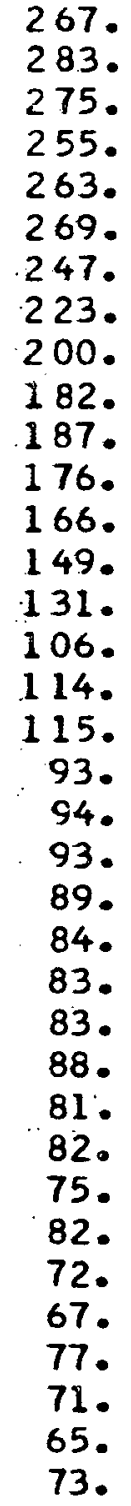 & 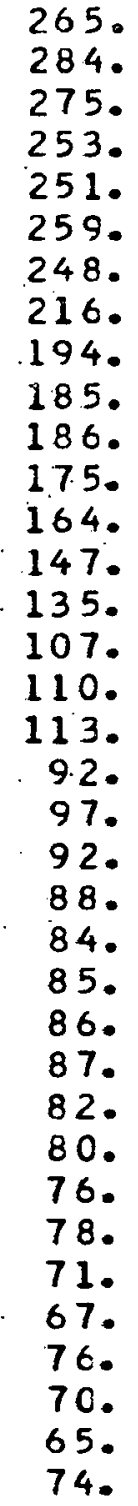 & 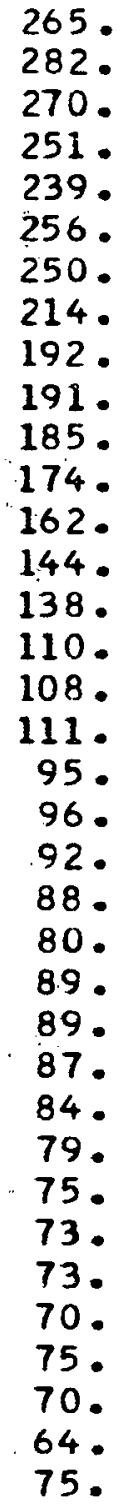 & 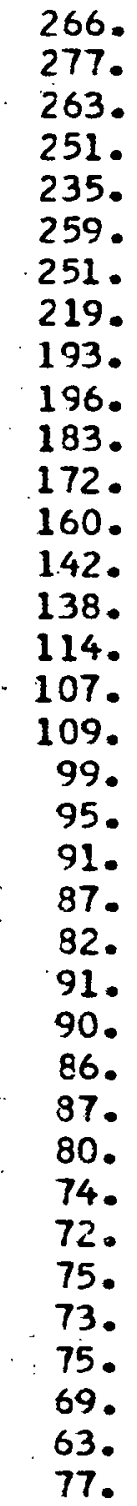 & $\begin{array}{l}268 . \\
271 . \\
256 . \\
255^{\circ} \\
243 . \\
264 . \\
250 . \\
224 . \\
194 . \\
195 . \\
182 . \\
171 . \\
158 . \\
140 . \\
132 . \\
117 . \\
109 . \\
108 . \\
103 . \\
95 . \\
91 . \\
86 . \\
85 . \\
91 . \\
89 . \\
86 . \\
89 . \\
80 . \\
74 . \\
73 . \\
76 . \\
77 . \\
74 . \\
68 . \\
59 . \\
79 .\end{array}$ \\
\hline
\end{tabular}

PAGE 16 


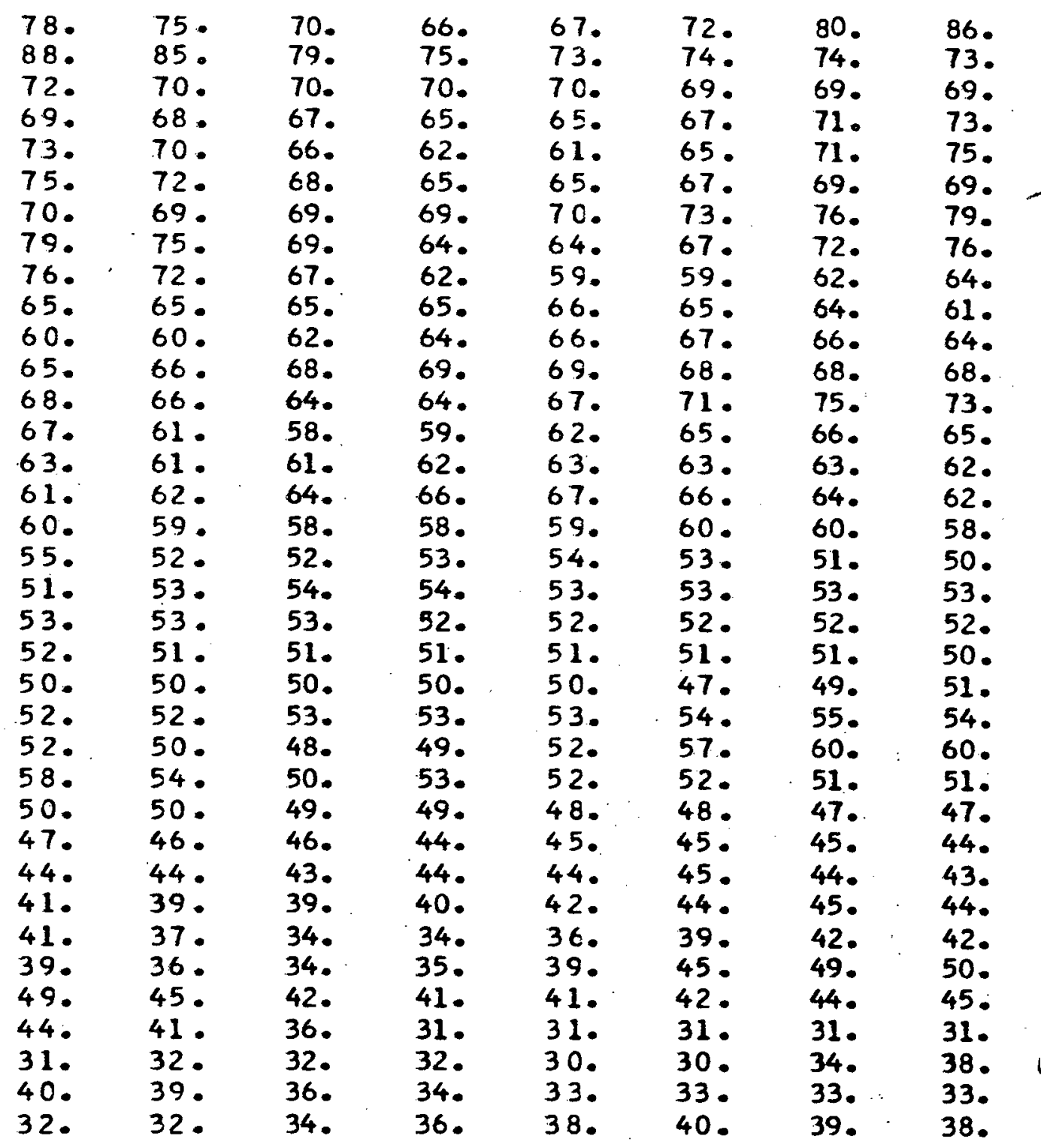

PAGE 17 


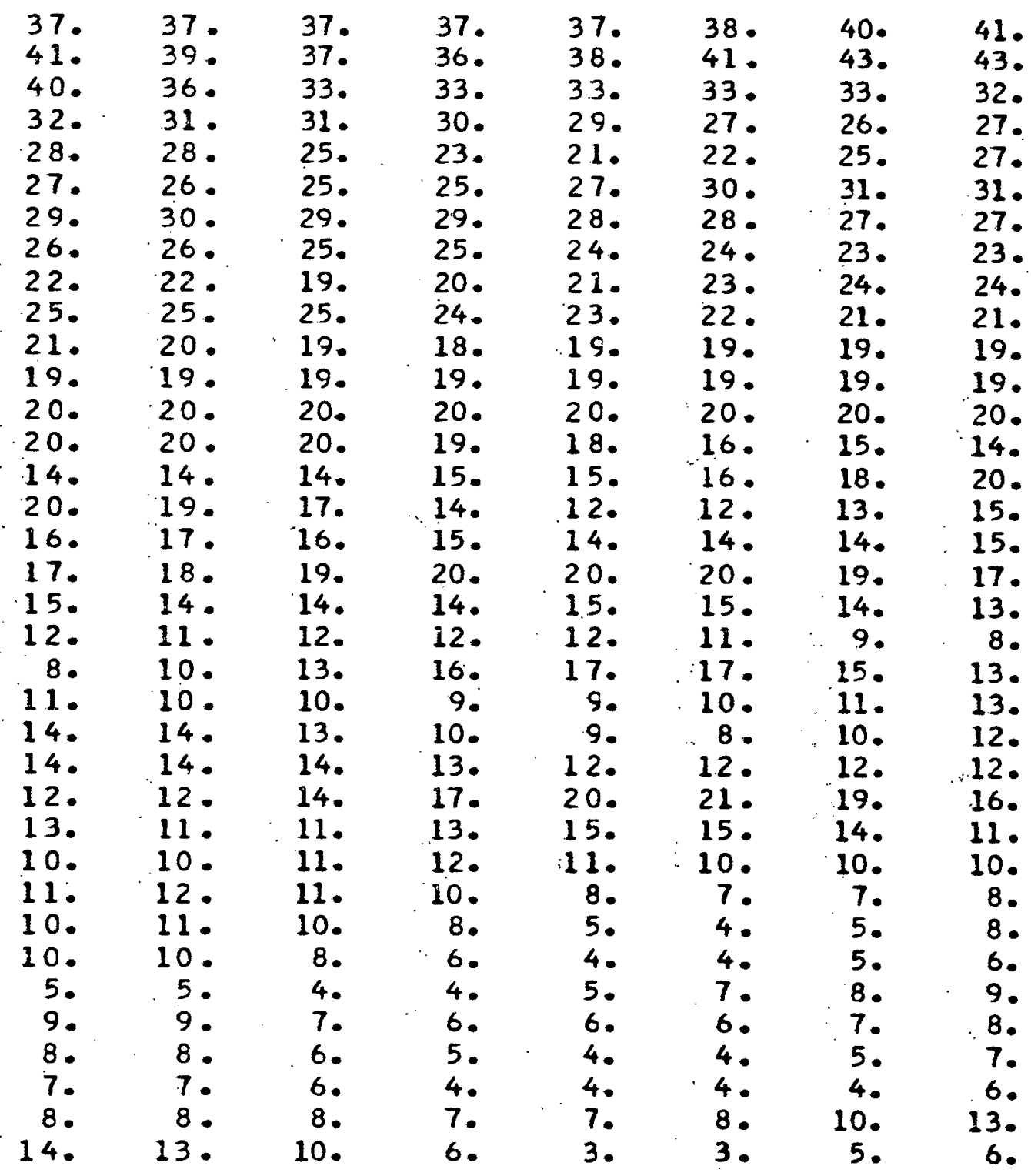


269

$\stackrel{2}{2}$

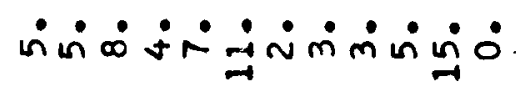

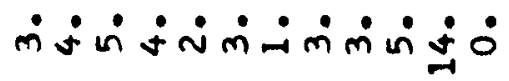

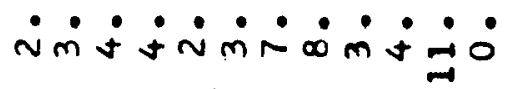

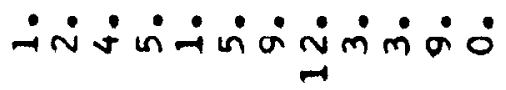

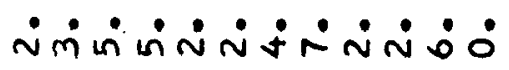

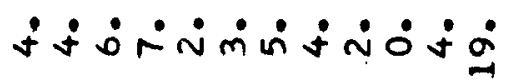

$\dot{0} \dot{\sim} \dot{0} \dot{m} \dot{m} \dot{m} \dot{\sim} \dot{\sim} \dot{s}$

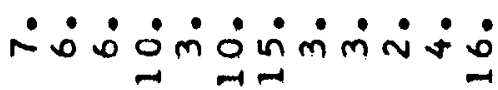




\section{PARAMETERS AND INPUT DATA FOR RUN 1054095 CHANNELS}

LOW CHANNEL NUMBER WHICH BEGINS ANALYSIS

(IMAX)

DIMENSION OF MATR IX 80

ENERGY PER CHANNEL NUMBER (KEV) 2.31538
CALIBP.ATION 1
$\mathrm{CH}=184$
$E=511.00$
CALIBRATI ON 2
$\mathrm{CH}=522$
$E=1293.60$
NUMBER OF ITERATICN PERFORMED 128

THE MEASURED COMPTON CONTINUUM VECTOR

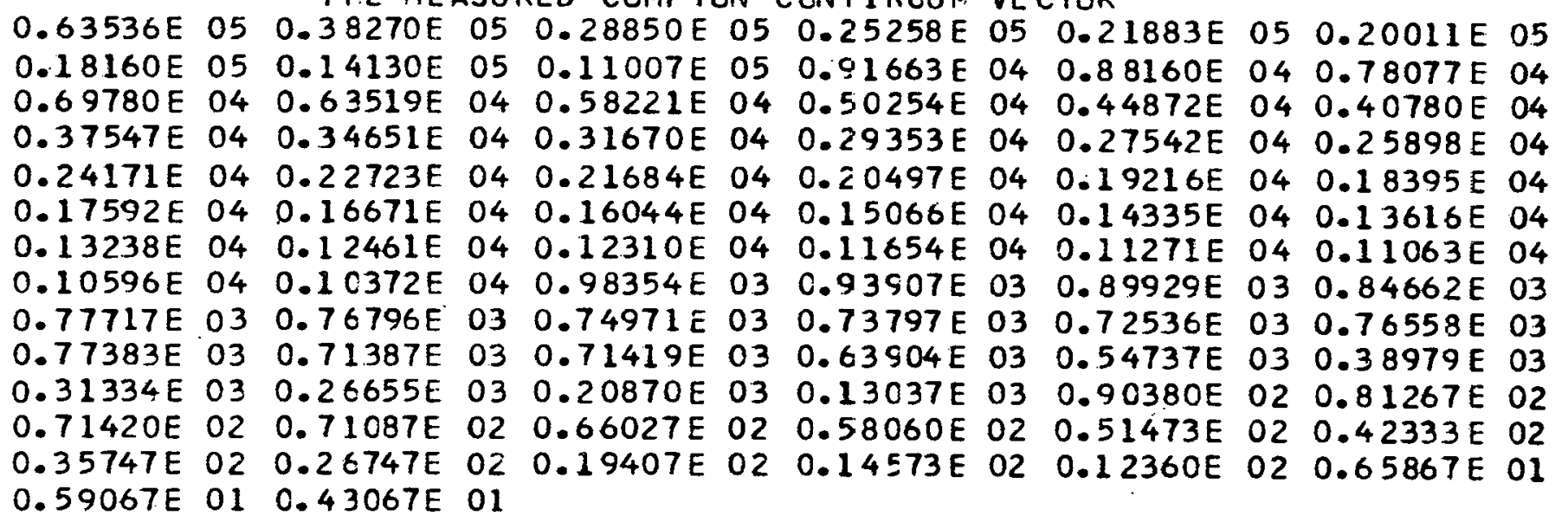


THE ENERGY ARRAY GF PHOTON IN KEV $\begin{array}{lllllllllllll}0.49943 E & 03 & 0.62776 E & 03 & 0.75269 E & 03 & 0.87549 E & 03 & 0.99685 E & 03 & 0.11172 E & 04\end{array}$ $\begin{array}{llllllllllllll}0.12368 E & 04 & 0.12558 E & 04 & 0.14743 E & 04 & 0.15925 E & 04 & 0.17104 E & 04 & 0.18280 E & 04\end{array}$ $\begin{array}{lllllllllllllll}0.19455 E & 04 & 0.20627 E & 04 & 0.21799 E & 04 & 0.22969 E & 04 & 0.24137 E & 04 & 0.25305 E & 04\end{array}$ $\begin{array}{llllllllllllll}0.26473 E & 04 & 0.27639 E & 04 & 0.28805 E & 04 & 0.29970 E & 04 & 0.31134 E & 04 & 0.32299 E & 04\end{array}$ $\begin{array}{llllllllllll}0.33462 E & 04 & 0.34626 E & 04 & 0.35789 E & 04 & 0.36951 E & 04 & 0.38114 E & 04 & 0.39276 E & 04\end{array}$ $\begin{array}{lllllllllllllll}0.40438 E & 04 & 0.41600 E & 04 & 0.42761 E & 04 & 0.43922 E & 04 & 0.45083 E & 04 & 0.46244 E & 04\end{array}$ $\begin{array}{lllllllllllll}0.47405 E & 04 & 0.48566 E & 04 & 0.49726 E & 04 & 0.50 \varepsilon 87 E & 04 & 0.52047 E & 04 & 0.53207 E & 04\end{array}$ $\begin{array}{llllllllllllll}0.54367 E & 04 & 0.55527 E & 04 & 0.56687 E & 04 & 0.57847 E & 04 & 0.59007 E & 04 & 0.60166 E & 04\end{array}$ $\begin{array}{llllllllllllll}0.61326 E & 04 & 0.62485 E & 04 & 0.63645 E & 04 & 0.64804 E & 04 & 0.65964 E & 04 & 0.67123 E & 04\end{array}$ $\begin{array}{lllllllllllllll}0.68282 E & 04 & 0.69441 E & 04 & 0.70600 E & 04 & 0.71760 E & 04 & 0.72919 E & 04 & 0.74078 E & 04\end{array}$ $\begin{array}{llllllllllllll}0.75237 E & 04 & 0.76395 E & 04 & 0.77554 E & 04 & 0.78713 E & 04 & 0.79872 E & 04 & 0.81031 E & 04\end{array}$ $\begin{array}{llllllllllllll}0.82190 E & 04 & 0.83348 E & 04 & 0.84507 E & 04 & 0.85666 E & 04 & 0.86824 E & 04 & 0.87983 E & 04\end{array}$ $\begin{array}{lllllllllllll}0.89142 \mathrm{E} & 04 & 0.90300 \mathrm{E} & 04 & 0.91459 \mathrm{E} & 04 & 0.92617 \mathrm{E} & 04 & 0.93776 \mathrm{E} & 04 & 0.94934 \mathrm{E} & 04\end{array}$ $0.96093 E \quad 04 \quad 0.97251 E \quad 04$

\section{THE UPPER TRI ANGULAR RESPONSE MATRIX}

$I=$

$0.43571 E$ E $00 \quad 0.93011 E-01 \quad 0.59835 E-01 \quad 0.45710 E-01 \quad 0.36446 E-01 \quad 0.29734 E-01$ $0.24689 E-01 \quad 0.20806 E-01 \quad 0.17759 E-01 \quad 0.15329 E-01 \quad 0.13361 E-010.11747 E-01$ $0.10407 E-01 \quad 0.92826 E-02 \quad 0.83308 E-02 \quad 0.75178 E-02 \quad 0.68180 E-02 \quad 0.62114 E-02$ $0.56823 E-02 \quad 0.52178 E-02 \quad 0.48081 E-02 \quad 0.44448 E-02 \quad 0.41212 E-02 \quad 0.38315 E-02$ $0.35715 E-02 \quad 0.33371 E-02 \quad 0.31248 E-02 \quad 0.29324 E-02 \quad 0.27571 E-02 \quad 0.25971 E-02$ $0.24506 E-02 \quad 0.23163 E-02$. $0.21926 E-02 \quad 0.20786 E-02 \quad 0.19732 E-02 \quad 0.18758 E-02$ $0.17853 E-02 \quad 0.17012 E-02 \quad 0.16229 E-02 \quad 0.15500 E-02 \quad 0.14818 E-02 \quad 0.14180 E-02$ $0.13583 E-02 \quad 0.13022 E-02 \quad 0.12496 E-02 \quad 0.12 C 01 E-02 \quad 0.11534 E-02 \quad 0.11095 E-02$ 0.10680 E-02 $0.10288 E-02 \quad 0.99171 E-03 \quad 0.95661 E-03 \quad 0.92331 E-03 \quad 0.89175 E-03$ $\begin{array}{lllllll}0.86178 E-03 & 0.83329 E-03 & 0.80616 E-03 & 0.78033 E-03 & 0.75578 E-03 & 0.73234 E-03\end{array}$ $0.70998 E-03 \quad 0.68862 E-03 \quad 0.66821 E-03 \quad 0.64 E 72 E-03 \quad 0.63004 E-03 \quad 0.61214 E-03$ $0.59503 E-03 \quad 0.57859 E-03 \quad 0.56286 E-03 \quad 0.54773$ E-03 $0.53330 E-03 \quad 0.51926 E-03$ $0.50594 E-03 \quad 0.49299 E-03 \quad 0.48063 E-03 \quad 0.46867 E-03 \quad 0.45720 E-03 \quad 0.44607 E-03$ $0.43542 E-03 \quad 0.42506 E-03$ 
0.26677 E $00 \quad 0.73779 E-01 \quad 0.47049 E-01 \quad 0.36148 E-01 \quad 0.29287 E-01 \quad 0.24320 E-01$ $0.20530 E-01 \quad 0.17556 E-01 \quad 0.15179 E-01 \quad 0.13249 E-01 \quad 0.11662 E-01 \quad 0.10341 E-01$ $0.92315 E-02 \quad 0.82904 E-02 \quad 0.74855 E-02 \quad 0.67919 E-02 \quad 0.61901 E-02 \quad 0.56647 E-02$ $0.52032 E-02 \quad 0.47959 E-02 \quad 0.44345 E-02 \quad 0.41124 E-02 \quad 0.38241 E-02 \quad 0.35650 E-02$ $0.33315 E-02 \quad 0.31200 E-02 \quad 0.29282 E-02 \quad 0.27534 E-02 \quad 0.25939 E-02$ $0.23137 E-02 \quad 0.21904 E-02 \quad 0.20766 E-02 \quad 0.19714 E-02 \quad 0.18742 E-02 \quad 0.17838 E-02$ $0.16999 \mathrm{E}-02 \quad 0.16217 \mathrm{E}-02 \quad 0.15489 \mathrm{E}-02$ ( $0.13015 E-02 \quad 0.12489 E-02 \quad 0.11994 E-02 \quad 0.11528 E-02$ $0.10284 E-02 \quad 0.99129 E-03 \quad 0.95622 E-03 \quad 0.92295 E-03$ $0.83299 \mathrm{E}-03 \quad 0.80588 \mathrm{E}-03 \quad 0.78008 \mathrm{E}-03 \quad 0.75554 \mathrm{E}-03$ $0.68842 E-03 \quad 0.66802 E-03 \quad 0.64854 E-03 \quad 0.62988 E-03$ $0.57846 E-03 \quad 0.56273 E-03 \quad 0.54760 E-03 \quad 0.53318 E-03$ $0.49289 E-03$ $0.4249 .9 E-03$

$0.48054 E-03 \quad 0.46858 E-03$

$0.45712 E-03$

$0.11090 E-02 \quad 0.10675 E-02$

$0.89141 E-030.86146 E-03$

$0.73212 E-03 \quad 0.70976 E-03$

$0.61199 E-030.59489 E-03$

$0.51915 E-030.50584 E-03$

$0.44599 E-03 \cdot 0.43534 E-03$

$I=$

$0.19113 \mathrm{E} 00$

$0.17560 E-01$

$0.82804 E-02$

$0.47928 E-02$

$0.31189 \mathrm{E}-02$

$0.21899 E-02$

$0.16215 \mathrm{E}-02$

$0.12488 \mathrm{E}-02$

$0.99121 E-03$

$0.80584 E-03$

$0.66799 E-03$

$0.56271 E-03$

$0.48052 E-03$

0.

0.14869 E 00 $0.15356 E-01$ $0.74990 E-02$ $0.44382 E-02$ $0.29298 E-02$ $0.20774 E-02$ $0.15494 E-02$
$0.618745-01$

5165E-01

$0.74773 E-02$

$0.44319 E-02$

$0.29272 E-02$

$0.20761 E-02$

$0.15487 \mathrm{E}-02$

$0.1 .1993 E-02$

$0.95615 E-03$

$0.78003 E-03$

$0.64851 E-03$

$0.54758 E-03$

$0.4 € 856 E-03$

$0.52625 E-01$

$0.13348 E-01$

$0.68022 \dot{E}-02$

$0.41155 E-02$

$0.27549 E-02$

$0.1 S 721 E-02$

$0.14813 E-02$
$0.13231 \mathrm{E}-01$

$0.67852 E-02$

$0.41102 E-02$

$0.27526 E-02$

0.19710 E-02

$0.14806 E-02$

$0.11527 E-02$

$0.92288 E-03$

$0.75550 E-0.3$

$0.62985 E-03$

$0.53317 \mathrm{E}-03$

$0.45711 E-03$

$0.34133 E-01$

$0.11722 \mathrm{E}-01$

$0.61982 E-02$

0.38267 E-02

$0.25951 E-02$

$0.18748 E-02$

$0.14175 \mathrm{E}-02$
$0.30141 E-01$

$0.11645 E-01$

$0.61846 \mathrm{E}-02$

$0.38222 \mathrm{E}-02$

$0.25931 E-02$

$0.18738 \mathrm{E}-02$

$0.14169 E-02$

$0.11 C 89 E-02$

C. $89135 E-03$

$0.73208 \mathrm{E}-03$

$0.61196 E-03$

$0.51914 \mathrm{E}-03$

$0.44597 E-03$

$0.26044 E-01$

$0.10380 E-01$

$0.56712 \mathrm{E}-02$

$0.35674 E-02$

$0.2448 .9 E-02$

$0.17845 E-02$

$0.13578 \mathrm{E}-02$
$0.24566 E-010.20592 E-01$

$0.10327 E-010.92193 E-02$

$0.56602 E-02 \quad 0.51995 E-02$

$0.35634 E-02 \quad 0.33302 E-02$

$0.24471 E-02 \quad 0.23132 E-02$

$0.17835 E-02 \quad 0.16996 E-02$

$0.13573 E-02 \quad 0.13013 E-02$

$0.10674 E-02 \quad 0.10283 E-02$

$0.86140 E-03 \quad 0.83294 E-03$

$0.70973 E-03 \quad 0.68839 E-03$

$0.59486 E-03 \quad 0.57843 E-03$

$0.50582 E-030.49288 E-03$

$0.43533 E-03 \quad 0.42498 E-03$

$0.21251 E-010.17897 E-01$

$0.92575 E-020.83088$ E-02

$0.52085 E-02 \quad 0.48003 E-02$

$0.33336 E-02 \quad 0.31218 E-02$

$0.23148 E-02 \quad 0.21913 E-02$

$0.17005 E-02 \quad 0.16223 E-02$

$0.13018 E-02,0.12493 E-02$ 


\begin{tabular}{|c|c|c|c|c|c|}
\hline $\begin{array}{l}.11998 E-02 \\
.95642 E-03 \\
.78021 E-03 \\
.64864 E-03 \\
.54767 E-03 \\
.46863 E-03\end{array}$ & $\begin{array}{c}0.11531 E-02 \\
0.92313 E-03 \\
0.75567 E-03 \\
0.62997 E-03 \\
0.53325 E-03 \\
0.45717 E-03 \\
I=\quad 5\end{array}$ & $\begin{array}{l}0.11093 E-02 \\
0.89158 E-03 \\
0.73224 E-03 \\
0.61207 E-03 \\
0.51922 E-03 \\
0.44603 E-03\end{array}$ & $\begin{array}{l}0.10677 E-02 \\
0.86162 E-03 \\
0.70588 E-03 \\
0.59497 E-03 \\
0.50590 E-03 \\
0.43539 E-03\end{array}$ & $\begin{array}{l}0.83315 E-03 \\
0.68853 E-0.3 \\
0.57853 E-03 \\
0.49295 E-03 \\
0.42503 E-03\end{array}$ & $\begin{array}{l}0.80603 E-03 \\
0.66813 E-03 \\
0.56281 E-03 \\
0.48059 E-03\end{array}$ \\
\hline $\begin{array}{l}0.12165 E-00 \\
0.13666 E-01 \\
0.68485 E-02 \\
0.41296 E-02 \\
0.27607 E-02 \\
0.19750 E-02 \\
0.14829 E-02 \\
0.11541 E-02 \\
0.92372 E-03 \\
0.75606 E-03 \\
0.63024 E-03 \\
0.53344 E-03 \\
0.45731 E-03\end{array}$ & $\begin{array}{l}0.47496 E-01 \\
0.11931 E-01 \\
0.62349 E-02 \\
0.38387 E-02 \\
0.26002 E-02 \\
0.18774 E-02 \\
0.14189 E-02 \\
0.11101 E-02 \\
0.89213 E-03 \\
0.73260 E-03 \\
0.61233 E-03 \\
0.51940 E-03 \\
0.44617 E-03\end{array}$ & $\begin{array}{l}0.30356 E-01 \\
0.10525 E-01 \\
0.57008 E-02 \\
0.35776 E-02 \\
0.24534 E-02 \\
0.17868 E-02 \\
0.13592 E-02 \\
0.10685 E-02 \\
0.86213 E-03 \\
0.71022 E-03 \\
0.59521 E-03 \\
0.50607 E-03 \\
0.43552 E-03\end{array}$ & $\begin{array}{l}0.23070 E-01 \\
0.93619 E-02 \\
0.52327 E-02 \\
0.33424 E-02 \\
0.23187 E-02 \\
0.17 C 25 E-02 \\
0.13030 E-02 \\
0.10293 E-02 \\
0.83362 E-03 \\
0.68885 E-03 \\
0.57876 E-03 \\
0.49312 E-03 \\
0.42516 E-03\end{array}$ & $\begin{array}{l}0.18808 E-01 \\
0.83865 E-02 \\
0.48203 E-02 \\
0.31294 E-02 \\
0.21948 E-02 \\
0.16242 E-02 \\
0.12504 E-02 \\
0.99218 E-03 \\
0.80647 E-03 \\
0.66843 E-03 \\
0.56302 E-03 \\
0.48075 E-03\end{array}$ & $\begin{array}{l}0.15871 E-01 \\
0.75583 E-02 \\
0.44549 E-02 \\
0.29364 E-02 \\
0.20806 E-02 \\
0.15511 E-02 \\
0.12008 E-02 \\
0.95705 E-03 \\
0.78063 E-03 \\
0.64892 E-03 \\
0.54788 E-03 \\
0.46878 E-03\end{array}$ \\
\hline $\begin{array}{l}0.10294 E \quad 00 \\
0.12334 E-01 \\
0.63003 E-02 \\
0.38592 E-02 \\
0.26088 E-02 \\
0.18816 E-02 \\
0.14213 E-02 \\
0.11115 E-02 \\
0.89301 E-03 \\
0.73319 E-03 \\
0.61273 E-03 \\
0.51969 E-03 \\
0.44638 E-03\end{array}$ & $\begin{array}{l}0.42728 E-01 \\
0.10798 E-01 \\
0.57531 E-02 \\
0.35950 E-02 \\
0.24609 E-02 \\
0.17906 E-02 \\
0.13613 E-02 \\
0.10698 E-02 \\
0.86296 E-03 \\
0.73 .077 E-03 \\
0.59559 E-03 \\
0.50634 E-03 \\
0.43572 E-03\end{array}$ & $\begin{array}{l}0.27459 E-01 \\
0.95560 E-02 \\
0.52751 E-02 \\
0.33573 E-02 \\
0.23254 E-02 \\
0.17060 E-02 \\
0.13050 E-02 \\
0.10305 E-02 \\
0.83439 E-03 \\
0.68937 E-03 \\
0.57912 E-03 \\
0.49337 E-03 \\
0.42535 E-03\end{array}$ & $\begin{array}{l}0.20805 E-01 \\
0.85290 E-02 \\
0.48551 E-02 \\
0.31423 E-02 \\
0.22008 E-02 \\
0.16273 E-02 \\
0.12522 E-02 \\
0.99328 E-03 \\
0.80719 E-03 \\
0.66891 E-03 \\
0.56336 E-03 \\
0.48 C 99 E-03\end{array}$ & $\begin{array}{l}0.16934 E-01 \\
0.76658 E-02 \\
0.44838 E-02 \\
0.29476 E-02 \\
0.20859 E-02 \\
0.15539 E-02 \\
0.12024 E-02 \\
0.95807 E-03 \\
0.78130 E-03 \\
0.64938 E-03 \\
0.54820 E-03 \\
0.46901 E-03\end{array}$ & $\begin{array}{l}0.14296 E-01 \\
0.69315 E-02 \\
0.41539 E-02 \\
0.27704 E-02 \\
0.19797 E-02 \\
0.14854 E-02 \\
0.11556 E-02 \\
0.92467 E-03 \\
0.75668 E-03 \\
0.63067 E-03 \\
0.53375 E-03 \\
0.45753 E-03\end{array}$ \\
\hline
\end{tabular}


$0.11260 E-01$ $0.58334 \mathrm{E}-02$ $0.36208 E-02$ $0.24718 E-02$ $0.17960 \mathrm{E}-02$ $0.13643 E-02$ $0.10716 E-02$ $0.86410 E-03$ $0.71154 E-03$ $0.59612 E-03$ $0.50672 E-03$ $0.43599 E-03$

$0.78783 E-01$ $0.10377 \mathrm{E}-01$ $0.5431 .7 E-02$ $0.34095 E-02$ $0.23480 E-02$ $0.17174 E-02$ $0.13113 E-02$ $0.10344 E-02$ $0.83685 E-03$ $0.69102 E-03$ $0.58027 \mathrm{E}-03$ $0.49420 E-03$ $0.42595 E-03$

$0.70532 E-01$ $0.96369 E-02$ $0.50832 E-02$ $0.32210 E-02$ $0.22354 E-02$ $0.16450 E-02$ $0.12622 E-02$ $0.99937 E-03$ $0.81110 E-0.3$
$0.98754 E-02 \quad 0.87588 E-02$

$0.53398 E-02 \quad 0.49078 E-02$

$0.33793 E-02$

$0.23350 E-02$

$0.17109 E-02$

$0.31613 E-02$

$0.22093 E-02$

$0.16317 \mathrm{E}-02$

$0.13077 E-02 \quad 0.12547 E-02$

$0.10322 E-02 \quad 0.99483 E-03$

$0.83546 E-03$
$0.80819 E-03$

$0.69009 E-03 \quad 0.66959 E-03$

$0.57962 E-03 \quad 0.56383 E-03$

$0.45373 E-03 \quad 0.48133 E-03$

$0.42561 E-03$

$1=$ 8

$0.35723 E-01 \quad 0.23247 E-01$

$0.91100 E-02$

$0.4 \$ 822 E-02$

$0.31871 E-02$

$0.22207 E-02$

$0.16375 E-02$

$0.12580 E-02$

$0.99684 E-03$

$0.80949 E-03$

$0.67046 E-03$

$0.56444 E-03$

$0.48177 E-03$

$0.80921 E-02$

$0.45881 . E-02$

$0.29862 \mathrm{E}-02$

$0.21036 \mathrm{E}-02$

$0.15632 \mathrm{E}-02$

$0.12078 E-02$

$0.96137 E-03$

$0.78344 E-03$

$0.65084 E-03$

$0.54922 E-03$

$0.46975 E-03$

I $=$ 9

$0.33060 E-01 \quad 0.21649 E-01 \quad 0.16340 E-01$

$0.84654 E-02$

$0.46702 E-02$

$0.30153 E-02$

$0.75273 E-02$

$0.43079 \mathrm{E}-02$

$0.28290 E-02$

$0.21166 E-02 \quad 0.20070 E-02$

$0.15699 E-02$

$0.14999 E-02$

$0.12116 E-02 \quad 0.11640 E-02$

$0.96371 E-03$

$0.92989 E-03$

$0.78495 E-03$
$0.78365 \mathrm{E}-02$ $0.45273 E-02$ $0.29640 E-02$ $0.20934 E-02$ $0.15579 E-02$ $0.12 C 47 E-02$ $0.95950 E-03$ $0.78223 E-03$ $0.65 \mathrm{CO1E}-03$ $0.54864 E-03$ $0.46 \$ 33 E-03$

$0.70616 E-02 \quad 0.64016 E-02$ $0.41901 E-02 \quad 0.38896 E-02$ $0.27847 E-02 \quad 0.26212 E-02$ $0.19864 E-02 \quad 0.18877 E-02$ $0.14890 \mathrm{E}-02 \quad 0.14246 \mathrm{E}-02$ $0.11577 E-02 \quad 0.11135 E-02$ $0.92600 E-03 \quad 0.89425 E-03$ $0.75756 E-03 \quad 0.73401 E-03$ $0.631 .26 E-03 \quad 0.61329 E-03$ $0.53417 E-03 \quad 0.52009 E-03$ $0.45784 E-03 \quad 0.44667 E-03$

$0.17556 E-01$ $0.72532 E-02$ $0.42405 E-02$ $0.28040 E-02$ $0.19 .955 . E-02$ $0.14938 \mathrm{E}-02$ $0.11605 E-02$ $0.92773 E-03$ $0.75869 E-03$ $0.63204 E-03$ $0.53471 E-03$ $0.45823 E-03$

$0.14241 E-01 \quad 0.12012 E-01$ $0.65488 E-020.59487 E-02$ $0.39318 E-02 \quad 0.36564 E-02$ $0.26381 E-02 \quad 0.24866 E-02$ $0.18957 E-02 \quad 0.18032 E-02$ $0.14289 E-02 \quad 0.13683 E-02$ $0.11160 E-02 \quad 0.10740 E-02$ $0.89586 E-03 \quad 0.86560 E-03$ $0.73507 E-03 \quad 0.71253 E-03$ $0.61402 E-030.59681 E-03$ $0.52060 E-03 \quad 0.50721 E-03$ $0.44705 E-03 \quad 0.43635 E-03$
$0.13236 E-010.11155 E-01$ $0.61087 E-020.55578 E-02$ $0.37034 E-02 \quad 0.34493 E-02$ $0.25056 E-02.0 .23647 E-02$ $0.18124 E-020.17256 E-02$ $0.13733 E-02 \quad 0.13159 E-02$ $0.10770 E+02 \quad 0.10371 E-02$ $0.86746 E-03 \quad 0.83859 E-03$ $0.71376 E-03 \quad 0.69217 E-03$ 
$0.67155 E-03$ $0.56520 E-03$ $0.48231 E-03$

$0.63859 E-01$

$0.90081 E-02$

$0.47782 \mathrm{E}-02$

$0.30522 E-02$

$0.21328 E-02$

$0.15781 \mathrm{E}-02$

$0.12162 \mathrm{E}-02$

$0.96654 E-03$

$0.78678 E-03$

$0.65308 \mathrm{E}-03$

$0.55079 E-03$

$0.47088 E-03$

$0.58348 E-01$

$0.84667 \mathrm{E}-02$

$0.45094 \mathrm{E}-02$

$0.29001 E-02$

$0.20388 E-02$

$0.15164 \mathrm{E}-02$

$0.11733 E-02$

$0.93563 E-03$

$0.76381 E-03$

0.63550 E-03

$0.53715 E-03$

$0.45999 E-03$

$0.53721 E-01$

$0.79953 E-02$

$0.42708 \mathrm{E}-02$

0.27627 E-02

$0.19529 E-02$

$0.14589 E-02$
$0.6 \leqq 185 E-03$

$0.54993 E-03$

$0.63300 E-03$

$0.53539 E-03$

$0.61492 E-03$

$0.52124 E-03$

$0.47027 \mathrm{E}-03$
$\mathrm{I}=10$

$0.30783 E-0 I$

$0.79153 E-02$

$0.43960 E-02$

$0.28608 E-02$

$0.20213 E-02$

$0.15074 E-02$

$0.11682 \mathrm{E}-02$

$0.93252 E-03$

$0.76180 E-03$

$0.63415 E-03$

$0.53620 E-03$

$0.45931 E-03$ $\mathrm{I}=$

$0.20281 E-01$

$0.70428 \mathrm{E}-02$

$0.40604 \mathrm{E}-02$

$0.26873 E-02$

$0.19188 \mathrm{E}-02$

$0.14412 E-02$

$0.11232 \mathrm{E}-02$

$0.90030 E-03$

$0.73797 E-03$

$0.61601 E-03$

$0.52200 E-03$

$0.44806 E-03$ 11

$0.28812 E-01 \quad 0.19092 E-01$

$0.74401 E-02$

$0.41532 E-02$

$0.27212 E-02$

$0.19343 E-02$

$0.14493 E-02$

$0.11278 E-02$

$0.90318 E-03$

$0.73985 E-03$

$0.66228 E-02$

$0.38407 E-02$

$0.25589 E-02$

$0.18376 \mathrm{E}-02$

$0.13868 E-02$

$0.10849 \mathrm{E}-02$

$0.87240 E-03$

$0.71701 E-03$

$0.61728 E-030.59988 E-03$

$0.52290 E-03 \quad 0.50938 E-03$

$0.44871 E-03 \quad 0.43794 E-03$ $I=$ 12

$0.27 C 87 E-01 \quad 0.18047 E-01$

$0.7 C 255 E-02$

$0.39371 E-02$

$0.25946 E-02$

$0.18541 E-02$

$0.13955 E-02$
$0.62551 E-02$

$0.24423 E-02$

$0.17629 E-02$

$0.13361 E-02$
$0.36444 E-02$
$0.15307 \mathrm{E}-01$

$0.63269 E-02$

$0.37639 E-02$

$0.25294 E-02$

$0.18238 \mathrm{E}-02$

$0.13794 E-02$

$0.10806 \mathrm{E}-02$

$0.86972 \mathrm{E}-03$

$0.71525 E-03$

$0.59868 E-03$

$0.50 \& 54 E-03$

$0.43732 E-03$

$0.14415 E-01$

$0.59536 E-02$

$0.35644 \mathrm{E}-02$

$0.24112 E-02$

$0.17482 \mathrm{E}-02$

$0.13282 E-02$

$0.10444 E-02$

$0.84317 \mathrm{E}-03$

$0.69521 E-03$

$0.58316 E-03$

$0.49625 E-03$

0.13635 E-01

$0.56258 \mathrm{E}-02$

$0.33 E 53 E-02$

$0.23034 E-02$

$0.16784 \mathrm{E}-02$

$0.12806 E-02$
$0.42746 E-03$
$0.59766 E-03 \quad 0.58107 E-03$

$0.50781 E-03 \quad 0.45477 E-03$

$0.43679 E-03 \quad 0.42637 E-03$

$0.12385 E-01 \quad 0.10430 E-01$

$0.57274 E-02 \quad 0.52175 E-02$

$0.35002 E-02 \quad 0.32642 E-02$

$0.23855 E-02 \quad 0.22538 E-02$

$0.17358 E-02 \quad 0.16541 E-02$

$0.13215 E-02 \quad 0.12673 E-02$

$0.10404 E-02 \quad 0.10024 E-02$

$0.84069 E-030.81306 E-03$

$0.69357 E-03 \quad 0.67286 E-03$

$0.58203 E-03 \quad 0.56610 E-03$

$0.49545 E-03 \quad 0.48296 E-03$

$0.42687 E-03$

$0.11654 E-01 \quad 0.98067 E-02$

$0.53941 E-02 \quad 0.49187 E-02$

$0.33183 E-02 \quad 0.30982 E-02$

$0.22762 E-02 \quad 0.21525 E-02$

$0.16652 E-02 \quad 0.15881 \mathrm{E}-02$

$0.12734 E-02 \quad 0.12218 E-02$

$0.10061 E-02 \quad 0.96991 E-03$

$0.81537 E-030.78893 E-03$

$0.67440 E-0.30 .65453 E-03$

$0.56717 E-03 \quad 0.55180 E-03$

$0.48372 E-030.47160 E-03$.

$0.11017 E-01 \quad 0.92642$ E-02

$0.51004 E-02 \quad 0.46545 E-02$

$0.31549 E-02 \quad 0.29485 E-02$

$0.21764 E+020.20598 E-02$

$0.15999 E-02 \quad 0.15270 E-02$

$0.12284 E-02 \quad 0.11793 E-02$ 
$0.11333 E-02$ $0.90655 E-03$ $0.74204 E-03$ $0.61876 E-03$ $0.52394 E-03$ $0.44946 E-03$

$0.4977 .9 E-01$ $0.75806 E-02$ $0.40577 E-02$ $0.26378 \mathrm{E}-02$ $0.18737 \mathrm{E}-02$ $0.14057 E-02$ 0.10957 E-02 $0.87913 \mathrm{E}-03$ $0.72139 E-03$ $0.60286 E-03$ $0.51148 E-03$ $0.43946 E-03$.

$0.46381 E-01$ $0.72124 \mathrm{E}-02$ $0.38665 E-02$ $0.25241 E-02$ $0.18006 \mathrm{E}-02$ $0.13560 E-02$ $0.10605 E-02$ $0.85323 \mathrm{E}-0.3$ $0.70180 E-03$ $0.58766 E-03$ $0.49943 E-03$ $0.42977 E-03$ $I=15$

$0.43421 E-010.22992 E-01 \quad 0.15542$ E-01 $0.11777 E-01$

$0.68833 E-02 \quad 0.6 C 453 E-02 \quad 0.53832 E-02 \quad 0.48447 E-02$ $0.36936 E-02$

C. 1 C899E-02 $0.10490 E-02$ $0.87553 E-03 \quad 0.84608 E-03$ $0.71905 E-03$ $0.60127 E-03$ $0.51036 E-03$ $0.69712 E-0.3$ $0.58447 E-03$ $0.49718 E-03$ $1=13$

$0.25564 E-010.17120 E-01$

$0.66601 E-02 \quad 0.59306 E-02$

$0.37437 E-02$

$0.24796 E-02 \quad 0.23358 E-02$

$0.17802 E-02 \quad 0.16939 E-02$

$0.13453 E-02 \quad 0.12889 E-02$

$0.10544 E-02 \quad 0.10153 E-02$

$0.84942 E-03 \quad 0.82118 E-03$

$0.65931 E-03 \quad 0.67824 E-03$

$0.58596 E-03 \quad 0.56981 E-03$

$0.49824 E-03 \quad 0.48560 E-03$

$0.42890 E-03$

$I=14$

$0.24208 E-010.16290 E-01$

$0.63357 E-02 \quad 0.56419 E-02$

$0.35693 E-02$

$0.23744 E-02$

$0.17120 E-02$

$0.12986 E-02$

$0.10210 E-02$

$0.82472 E-03$

$0.68056 E-03$

$0.57141 E-03$

$0.33088 E-02$

$0.22384 E-02$

$0.16300 E-02$

$0.12448 E-02$

$0.98362 E-03$

$0.79762 E-03$

$0.66031 E-03$

$0.55579 E-03$

$0.47445 E-03$

$0.12330 E-01$

$0.50769 E-02$

$0.30784 E-02$

$0.21142 E-02$

$0.15540 E-02$

$0.11943 E-02$

$0.94829 E-03$

$0.77191 \mathrm{E}-03$

$0.64 C 93 E-03$

$0.54 C 91 \mathrm{E}-03$

$0.46269 E-03$

$0.34116 E-02$
$0.31644 E-02$
$0.29458 E-02$
$0.97385 E-03 \quad 0.93927 E-0.3$

$0.79144 E-03 \quad 0.76615 E-03$

$0.65620 E-03 \quad 0.63708 E-0.3$

$0.55296 E-03 \quad 0.53824 E-03$

$0.47243 E-03 \quad 0.46078 E-03$

$0.10455 E-01 \quad 0.87871 E-02$

$0.48397 E-02 \quad 0.44191$ E-02

$0.30072 E-02 \quad 0.28128 E-02$

$0.20848 E-02 \quad 0.19749 E-02$

$0.15395 E-02 \quad 0.14702 E-02$

$0.11863 E-02 \quad 0.11397 E-02$

$0.94348 E-03 \quad 0.91044 E-03$

$0.76884 E-03 \quad 0.74455 E-03$

$0.63888 E-03 \quad 0.62045 E-03$

$0.53950 E-03 \quad 0.52512 E-03$

$0.46168 E-03 \quad 0.45032 E-03$

$0.99559 E-02 \quad 0.83636 E-02$

$0.46066 E-02 \quad 0.42084 E-02$

$0.28732 E-02 \quad 0.26895 E-02$

$0.20008 E-02 \quad 0.18966 E-02$

$0.14833 E-020.14175 E-02$

$0.11471 E-02 \quad 0.11025 E-02$

$0.91489 E-03 \quad 0.88324 E-03$

$0.74740 E-030.72406 E-03$

$0.62237 E-03 \quad 0.60466 E-03$

$0.52646 E-030.51275 E-03$

$0.45128 E-03 \quad 0.44037 E-03$

$0.95082 E-02 \quad 0.79844 E-02$ $0.43972 E-02 \quad 0.40186 E-02$ $0.27512 E-020.25770 E-02$ 
$0.24200 E-02$ $0.17330 E-02$ $0.13097 \mathrm{E}-02$ $0.10274 E-02$ $0.82872 E-03$ $0.68319 E-03$ $0.57320 E-03$ $0.48800 E-03$

$0.40819 E-01$ $0.65867 E-02$ $0.35369 E-02$ $0.23245 E-02$ $0.16704 \mathrm{E}-02$ $0.12664 \mathrm{E}-02$ $0.99624 E-03$ $0.80552 E-03$ $0.66550 E-03$ $0.55935 E-03$ $0.47698 E-03$

$0.38514 \mathrm{E}-01$ 0.63180 E-02 $0.33941 E-02$ $0.22364 E-02$ $0.16122 \mathrm{E}-02$ $0.12258 \mathrm{E}-02$ $0.96684 E-03$ $0.783585-03$ $0.64865 E-03$ $0.54624 E-03$ $0.46648 \mathrm{E}-03$

0.36457 E-OI $0.60729 E-02$ $0.32633 E-02$
$0.22780 E-02$ $0.16488 E-02$ $0.12549 E-02$ $0.98955 E-03$ $0.80134 E-03$ $0.6 \leqslant 276 E-0.3$ $0.55748 E-03$ $0.47565 E-03$ $1=$

$0.21896 E-010.14864 E-010.11275 E-01$ $0.57838 \mathrm{E}-02$ $0.32683 E-02$ $0.21892 E-02$ $0.15901 E-02$ $0.12140 E-02$ $0.95992 E-0.3$ $0.77925 E-03$

$0.64579 E-03$

$0.54427 E-03$ $0.45509 E-0$ $\mathrm{I}=$ 17

0.2 C901E-01 $0.14246 E-01 \quad 0.10819 E-01$ $0.55467 E-02 \quad 0.49385 E-02$ $0.31374 E-02 \quad 0.29125 E-02$ $0.21076 E-02 \quad 0.19903 E-02$ $0.15354 E-02 \quad 0.14643 E-02$ $0.11757 E-02 \quad 0.11286 E-02$ $0.93199 E-03 \quad 0.89904 E-03$ $0.75827 E-03 \quad 0.73418 E-03$ $0.62961 E-03 \quad 0.61146 E-03$ $0.53147 E-03 \quad 0.51748 E-03$ $0.45486 E-03 \quad 0.44377 E-03$ $1=$ 18

$0.19995 E-01 \quad 0.13679 E-01$

$0.53308 E-02 \quad 0.47460 E-02$ $0.30173 E-02$
$0.20311 E-02$ $0.14983 E-02$ $0.11554 \mathrm{E}-02$ $0.91593 \mathrm{E}-03$ $0.75062 E-03$ $0.62453 E-03$ $0.52796 E-03$ $0.45235 E-03$ $0.46352 E-02$ $0.28247 E-02$ $0.19543 E-02$ $0.14466 E-02$ $0.11188 \mathrm{E}-02$ $0.89316 E-03$ $0.73643 E-03$ $0.60895 E-03$ $0.51574 E-03$ $0.44252 E-03$ $0.27140 E-02$ $0.18 \varepsilon 33 \mathrm{E}-02$ $0.13582 E-02$ $0.10845 \mathrm{E}-02$ $0.86785 E-03$ $0.71125 \mathrm{E}-03$ $0.59404 \mathrm{E}-03$ $0.50391 E-03$ $0.43300 E-03$

$0.10401 E-01$ $0.42717 \mathrm{E}-02$ $0.26121 E-02$ $0.10 \notin 19 E-01$
$0.19233 E-02 \quad 0.18242 E-02$ $0.14310 E-02 \quad 0.13683 E-02$ $0.11101 E-02 \quad 0.10676 E-02$ $0.88791 E-03 \quad 0.85755 E-03$ $0.72706 E-03 \quad 0.70461 E-03$ $0.60669 E-03 \quad 0.58956 E-03$ $0.51416 E-03 \quad 0.50077 E-03$ $0.44139 E-03 \quad 0.43074 E-03$

C. $91038 E-02 \quad 0.76427 E-02$ $0.4207 .9 E-02 \quad 0.38466 E-02$ $0.26397 E-02 \quad 0.24739 E-02$ $0.18516 E-02 \quad 0.17573 E-02$ $0.13823 E-020.13224 E-02$ $0.10755 E-02 \quad 0.10347 E-02$ $0.86241 E-03 \quad 0.83322 E-03$ $0.70775 E-03 \quad 0.68612 E-03$ $0.59169 E-03 \quad 0.57520 E-03$ $0.50226 E-03 \quad 0.48941 E-03$ $0.43181 E-03$

\section{$0.87363 E-02 \quad 0.73327 E-02$} $0.40357 E-02 \quad 0.36902 E-02$ $0.25373 E-020.23790 E-02$ $0.17853 E-02 \quad 0.16952 E-02$ $0.13368 E-02 \quad 0.12794 E-02$ $0.10429 E-02 \quad 0.10038 E-02$ $\begin{array}{lll}0.83826 E-03 & 0.81019 E-03\end{array}$ $0.68939 E-030.66856 E-03$ $0.57741 E-03 \quad 0.56144 E-03$ $0.49096 E-030.47845 E-03$

$0.84005 E-02 \quad 0.70499 E-02$ $0.38788 E-02 \quad 0.35472 E-02$ $0.24430 E-020.22915 E-02$ 
$0.21553 E-02$ $0.15579 E-02$ $0.11878 E-02$ $0.93911 E-03$ $0.76274 E-03$

$0.63257 E-03$

0.53351 E-03

$0.45631 E-03$

$0.34610 \mathrm{E}-01$

$0.58486 E-02$

$0.31432 E-02$

$0.20801 E-02$

$0.15073 E-02$

$0.11520 E-02$

$0.91291 E-03$

$0.74295 E-03$

$0.61728 E-03$

$0.52151 E-03$

$0.44665 E-03$

$0.32943 E-01$

$0.56423 E-02$

$0.30325 E-02$

$0.20102 E-02$

$0.14599 E-02$

$0.11183 E-02$

$0.88810 E-03$

$0.72413 E-03$

$0.60265 E-03$

$0.50988 E-03$

$0.43727 \mathrm{E}-03$

$I=.21$

$0.31430 E-01 \quad 0.17701 E-01$

$0.54514 E-02 \quad 0.47834 E-02$

$0.29301 E-02$

$0.20319 E-02$

$0.14844 E-02$

$0.11396 \mathrm{E}-02$

$0.90561 E-03$

$0.73835 E-03$

$0.51940 E-03$

$0.44514 E-03$

$0.19166 E-01$

$0.51332 E-02$

$0.29071 E-02$

$0.19617 E-02$

$0.11058 E-02$

$0.88064 \mathrm{E}-03$

$0.71941 E-03$

$0.59951 E-03$

$0.5 C 771 E-03$

$0.43572 E-03$

$1=$

$0.18404 \mathrm{E}-01$

$0.49512 E-02$

$0.28052 \mathrm{E}-02$

$0.85697 E-03$

$0.27109 E-02$
$0.61423 E-03$

$0.14368 \mathrm{E}-02$

$0.19197 E-02$

$0.14163 E-02$

$0.10945 E-02$

$0.87391 E-03$

$0.71513 E-03$

$0.59664 E-03$

$0.50572 E-03$

$0.43430 E-03$

$0.13157 \mathrm{E}-01$

$0.45694 \mathrm{E}-02$

$0.27005 E-02$

$0.18542 \mathrm{E}-02$

$0.13715 \mathrm{E}-02$

$0.10624 E-02$

$0.85009 E-03$

$0.69700 \mathrm{E}-03$

$0.58254 E-03$

$0.49455 \mathrm{E}-03$

$0.18174 E-02$

$0.13531 \mathrm{E}-02$

$0.10521 E-02$

$0.84386 \mathrm{E}-03$

$0.69301 \mathrm{E}-03$

$0.57985 E-03$

$0.49267 E-03$

$0.10016 E-01$

$0.41129 E-02$

$0.25181 \mathrm{E}-02$

$0.17561 \mathrm{E}-02$

$0.13107 \mathrm{E}-02$

$0.10216 E-02$

$0.82114 E-03$

$0.67567 \mathrm{E}-03$

$0.56626 \mathrm{E}-03$

$0.48184 E-03$

$0.17236 E-02 \quad 0.16374 E-02$

$0.12941 E-02 \quad 0.12391 E-02$

$0.10122 E-02 \quad 0.97458 E-03$

$0.81538 E-03 \quad 0.78840 E-03$

$0.67194 E-03 \quad 0.65181 E-03$

$0.56374 E-03 \quad 0.54840 E-03$

$0.48007 E-03 \quad 0.46801 E-03$

$0.80922 E-020.67905 E-02$

$0.37348 E-02 \quad 0.34160 E-02$

$0.23558 E-02 \quad 0.22108 E-02$

$0.16662 \mathrm{E}-020.15835 \mathrm{E}-02$

$0.12541 E-02 \quad 0.12014 E-02$

$0.98321 E-030.94705 E-03$

$0.79374 E-03 \quad 0.76770 E-03$

$0.65530 E-03 \quad 0.63583 E-03$

$0.55077 E-03 \quad 0.53575 E-03$

$0.46969 E-030.45790 E-03$

$0.12676 \mathrm{E}-01$

$0.44073 E-02$

$0.96611 \mathrm{E}-02$

$0.39668 E-02$

$0.26065 E-02$

$0.24310 E-02$

$0.16990 E-02$

(1) $0.17933 E-02$

$0.13294 E-02$

$0.12709 E-02$

$0.99281 \mathrm{E}-03$

$0.10321 \mathrm{E}-02$

$0.79563 E-03$

$0.65913 E-03$

$0.67977 E-03$

$0.55337 E-03$

$0.47152 \mathrm{E}-03$

$0.78076 E-02 \quad 0.65516 E-02$

$0.36022 E-02 \quad 0.32950 E-02$

$0.22753 E-02 \quad 0.21358 E-02$

$0.16126 E-02 \quad 0.15332 E-02$

$0.12166 E-02 \quad 0.11658 E-02$

$0.95585 E-03 \quad 0.92101 E-03$

$\begin{array}{lll}0.77316 E-03 & 0.74803 E-03\end{array}$

$0.63941 E-03 \quad 0.62063 E-03$

$0.53819 E-03 \quad 0.52381 E-03$

$0.45963 E-030.44828 E-03$

$0.48378 E-03$

$0.75442 E \leftarrow 02 \quad 0.63309$ E-02

$0.12229 E-01 \quad 0.93317 E-02$

$0.42575 E-02$

$0.38319 \mathrm{E}-02$

$0.25192 E-02$

$0.23505 E-02$

$0.34797 E-02 \quad 0.31834$ E-02

$0.22003 E-02 \quad 0.20660 E-02$

PAGE 28 
$0.19451 E-02$

$0.14156 E-02$

$0.10866 E-02$

$0.86457 E-03$

$0.70623 E-03$

$0.58871 E-03$

$0.49883 E-03$

$0.30051 E-01$

$0.52746 \mathrm{E}-02$

$0.28350 E-02$

$0.18844 \mathrm{E}-02$

$0.13738 E-02$

$0.10566 E-02$

$0.84225 E-03$

$0.68919 E-03$

$0.57534 \mathrm{E}-03$

$0.48817 \mathrm{E}-03$

$0.28789 E-01$

$0.28789 E-01$
$0.51101 E-02$

$0.27463 E-02$

$0.18277 E-02$

$0.13346 E-02$

$0.10283 E-02$

$0.82110 E-03$

$0.67292 E-03$

$0.56266 E-03$

$0.47800 E-03$

$0.27628 E-01$

$0.49565 E-02$

$0.26639 E-02$

$0.17744 \mathrm{E}-02$

$0.12978 E-02$

$0.10015 E-02$
$0.18358 E-02$

$0.13503 E-02$

$0.10437 E-02$

$0.83452 E-03$

$0.68427 E-03$

$0.57205 E-03$

$0.48588 E-03$
$1=22$

$0.17051 E-01 \cdot 0.11814$ E-01 0.90255 E-02

$0.46278 E-02 \quad 0.41187 E-02 \quad 0.37 C 68 E-02$

$0.26232 E-02 \quad 0.24384 E-02 \quad 0.22754 E-02$

$0.17790 E-02$

$0.13109 E-02$

$0.10152 E-02$

$0.1 .6831 \mathrm{E}-02$

$0.12527 \mathrm{E}-02$

0.15957 E-02

$0.11985 \mathrm{E}-02$

$0.97640 E-03 \quad 0.93987 E-03$

$0.81326 E-03 \quad 0.78579 E-03$

$0.66791 E-03$

$0.55930 E=03$ $0.47566 E-03$
$.1=23$

$0.64762 E-030.62830 E-03$

$0.54376 E-03 \quad 0.52506 E-03$

$0.46355 E-03$

$0.45199 E-03$

$0.87400 \mathrm{E}-02$

$\begin{array}{lll}0.16447 E-01 & 0.11428 E-01 & 0.87400 E-02 \\ 0.44831 E-02 & 0.39896 E-02 & 0.35907 E-02\end{array}$

$\begin{array}{llll}0.25418 E-02 & 0.23629 E-02 & 0.22053 E-02\end{array}$

$0.17258 E-02 \quad 0.16333 E-02 \quad 0.15488 E-02$

$0.12740 E-02$

$0.12176 \mathrm{E}-02$

$0.11653 \mathrm{E}-02$

$0.98833 E-03$

$0.79303 E-03$

$0.95079 E-03$

$0.76644 \mathrm{E}-03$

$0.91547 E-03$

$0.74123 E-03$

$0.65229 E-03$

$0.63266 E-03$

$0.53202 E-03$

$0.61390 \mathrm{E}-03$

$0.51761 E-03$

$0.46576 E-03 \quad 0.45408 E-03 \quad 0.44276 E-03$ $I=24$

$0.15885 E-01 \quad 0.11066 E-01 \quad 0.84725 E-02$

$0.43481 E-02 \quad 0.38695 E-02 \quad 0.34823$ E-02

$0.24656 E-02 \quad 0.22924 E-02 \quad 0.21398 E-02$

$0.16759 E-02 \quad 0.15864 E-02 \quad 0.15 C 48$ E-02

$0.12390 E-02 \quad 0.11846 E-02 \quad 0.11340 E-02$

$0.96282 E-03$

$0.92650 E-03$

$0.89229 E-03$

$0.68581 E-02 \quad 0.57567 E-02$

$0.31624 E-02 \quad 0.28933 E-02$

$0.20044 E-020.18834 E-02$

$0.14300 E+020.13611 E-02$

$0.10868 E-02 \quad 0.10427 E-02$

$0.86005 E-030.82968 E-03$ 
$0.80096 E-03$ $0.65737 E-03$

$0.55033 E-03$

$0.46814 E-03$

$0.26559 E-01$

0.48127 E-02

$0.25866 E-02$

$0.17244 \mathrm{E}-02$

$0.12629 E-02$

$0.97608 E-03$

$0.7817 .9 E-03$

$0.64256 E-03$

$0.53870 E-03$

$0.45876 E-03$

$0.25570 E-01$ 0.46780 E-02

$0.25141 E-02$

$0.16773 E-02$

0.12300 E-O2

$0.95195 E-03$

$0.76352 E-03$

$0.62835 E-03$

$0.52740 E-03$ $0.44965 E-03$

$0.24651 E-01$

$0.45512 E-02$

$0.24460 E-02$

0.16331 E-02

$0.11988 \mathrm{E}-02$

$0.92899 E-03$

$0.74610 E-03$

$0.61480 E-03$

$0.51667 \mathrm{E}-03$ $\begin{array}{lll}0.77378 E-03 & 0.74802 E-03\end{array}$

$0.63741 E-03 \quad 0.61833 E-03$

$0.53523 E-03 \quad 0.52062 E-03$

$0.45634 E-03 \quad 0.44489 E-03$ $\mathrm{I}=$ 25

$0.15362 E-01 \quad 0.10727 E-01$

$0.42220 E-02 \quad 0.37570 E-02$

$0.23942 E-02 \quad 0.22263 E-02$

$0.16290 E-02 \quad 0.15424 E-02$

$0.12061 E-020.11534 E-02$

$0.93862 E-03 \quad 0.90340 E-03$

$0.75544 E-03 \quad 0.73047 E-03$

$0.62314 E-03 \quad 0.60467 E-03$

$0.52389 E-03 \quad 0.50979 E-03$

$0.44718 E-03$

$$
I=26
$$

$0.14871 E-01,0.10409 E-01$

$0.41037 E-02 \quad 0.36515 E-02$

$0.23273 E-02 \quad 0.21643 E-02$

$0.15849 E-02 \quad 0.15009 E-02$

$0.11749 E-02 \quad 0.11238 E-02$

$0.91561 E-03 \quad 0.88146 E-03$

$0.73796 E-03 \quad 0.71375 E-03$

$0.6 C 954 E-03 \quad 0.59155 E-03$

$0.51310 E-03 \quad 0.49933 E-03$

$0.72360 E-03$

$0.60018 E-03$

$0.50672 E-03$

$0.70042 E-03 \quad 0.67837 E-03$

$0.58280 E-03 \quad 0.56630 E-03$

$0.49333 E-03 \quad 0.48053 E-03$

$0.82220 E-02$

$0.33 \varepsilon 20 E-02$

$0.20785 E-02$

$0.14633 E-02$

$0.11044 E-02$

$0.87 C 27 \mathrm{E}-03$

$0.70681 E-03$

$0.58700 E-03$

$0.49622 E-03$

$0.66582 E-02 \quad 0.55896 E-02$ $0.30703 E-02 \quad 0.28094 E-02$

$0.19473 E-02 \quad 0.18299 E-02$

$0.13908 E-02 \quad 0.13243 E-02$

$0.10587 E-02 \quad 0.10160$ E-02

$0.83909 E-03 \quad 0.80963 E-03$

$0.68431 E-03.0 .66289 E-03$

$0.57024 E-03 \quad 0.55403 E-03$

$0.48325 E-03 \quad 0.47071 E-03$

$0.79 \varepsilon 64 E-02$
$0.32859 E-02$

$0.32859 . E-02$

$0.20209 E-02$
$0.14242 E-02$

$0.10763 \mathrm{E}-02$

$0.84938 . E-03$

$0.69075 E-03$

$0.57450 E-03$

$0.48619 E-03$

$0.64702 E-02 \quad 0.54327 E-02$

$0.29842 E-02 \quad 0.27305 E-02$

$0.18935 E-02 \quad 0.17797 E-02$

$0.13541 E-020.12895 E-02$

$0.10320 E-02 \quad 0.99067 E-03$

$0.81911 E-03 \quad 0.79053 E-03$

$0.66889 E-03 \quad 0.64814 E-03$

$0.55802 E-030.54245 E-03$

$0.47348 E-03 \quad 0.46138 E-03$

$$
I=27
$$

$0.14412 E-010.10110 E-01$

$0.39924 E-02 \quad 0.35523 E-02$

$0.22644 E-02 \quad 0.21061 E-02$

$0.15432 E-02 \quad 0.14616 E-02$

$0.11454 \mathrm{E}-02 \quad 0.10958 \mathrm{E}-02$

$0.89372 E-030.86063 E-03$

$0.72129 E-03 \quad 0.69776 E-03$

$0.59647 E-03 \quad 0.57910 E-03$

$0.50268 E-03 \quad 0.48935 E-03$

$0.77644 E-02$

$0.31969 \mathrm{E}-02$

$0.19666 E-02$

$0.13873 E-02$

$0.10497 \mathrm{E}-02$

$0.82546 E-03$

$0.67540 \mathrm{E}-03$

$0.56233 E-03$

$0.47646 E-03$ 
$I=$ 28

$0.23798 E-01 \quad 0.13980 E-0$ $0.44316 E-02$ $0.23819 E-02$ $0.15911 E-02$ $0.11692 \mathrm{E}-02$ $0.90715 E-03$ $0.72949 E-03$ $0.60178 E-03$ $0.50629 E-03$

$0.23001 E-01$

$0.43184 E-02$

$0.23214 E-02$

$0.15515 \mathrm{E}-02$

$0.11412 \mathrm{E}-02$

$0.88641 E-03$

$0.71360 E-03$

$0.58942 E-03$

$0.49638 E-03$

$0.22257 E-01$

0.42118 E-02

$0.22640 E-02$

0.15140 E-02

$0.11145 E-02$

$0.86660 E-03$

0.69837 E-03

$0.57737 E-03$

$0.48675 E-03$

$0.13573 E-01$

$0.13190 E-01$

$0.41104 E-02 \quad 0.36061 E-0.2$

$0.22099 E-02$

$0.14784 \mathrm{E}-02$

$0.10892 \mathrm{E}-02$
$0.38873 E-02$

$0.22052 E-02$

$0.15038 E-02$

$0.11173 E-02$

$0.87294 E-03$

$0.70535 E-03$

$0.58407 E-03$

$0.49274 E-03$

$$
\mathrm{I}=
$$$$
29
$$

$0.37886 E-02$

$0.21492 E-02$

$0.14667 E-02$

$0.10907 E-02$

$0.85311 E-03$

$0.69009 E-03$

$0.57198 E-03$

$0.48308 E-03$

$$
\text { I }=
$$$$
30
$$

$0.36949 E-02$

$0.20963 E-02$

$0.14313 E-02$

$0.10654 \mathrm{E}-02$

$0.83419 E-03$

$0.67553 E-03$

$0.56058 E-03$

$0.47388 E-03$

$$
\mathrm{I}=
$$$$
31
$$

$0.98273 E-02$

$0.34592 E-02$

$0.20510 E-02$

$0.14247 \mathrm{E}-02$

$0.10692 E-02$

0.84077 E-03

$0.68245 E-03$

$0.56698 E-03$

$0.47965 E-03$

$0.95604 E-02$

0.33711 E-02

$0.19992 E-02$

$0.13895 \mathrm{E}-02$

$0.10439 E-02$

$0.82182 E-03$

$0.66785 E-03$

$0.55553 E-03$

$0.47042 E-03$

$0.93084 E-02$

0.32877 E-02

$0.19500 E-02$

$0.13563 E-02$

$0.10199 E-02$

$0.80374 E-03$

$0.65383 E-03$

$0.54440 E-03$

$0.46145 E-03$

$0.90693 E-02$
$0.32088 E-02$

$0.19036 E-02$

$0.20461 E-02$

$0.13978 E-02$

$0.10414 \mathrm{E}-02$

$0.13247 E-02$

$0.99697 E-03$
0.75548 E-02 $0.61264 E-02 \quad 0.51461 E-02$

$0.31128 E-02 \quad 0.28268 E-02 \quad 0.25866 E-02$

$0.19 .154 E-02 \quad 0.17951 E-02 \quad 0.16877 E-02$

$0.13523 E-02 \quad 0.12862 E-02 \quad 0.12253 E-02$

$0.10244 E-02 \quad 0.98270 E-03 \quad 0.94368 E-03$

$0.81048 E-03 \quad 0.78192 E-03 \quad 0.75496 E-03$

$0.66 C 76 E-03 \quad 0.64010 E-03 \quad 0.62049 E-03$

$0.55084 E-03 \quad 0.53528 E-03 \quad 0.52050 E-03$

$0.46719 E-03 \quad 0.45514 E-03$

$0.73570 E-02 \quad 0.59685 E-02 \quad 0.50144 E-02$

$0.30335 E-02 \quad 0.27549 E-02 \quad 0.25208 E-02$

$0.18671 E-02 \quad 0.17501 E-02 \quad 0.16455 E-02$

$0.13193 E-02 \quad 0.12549 E-02 \quad 0.11958 E-02$

$0.10 C 04 E-02 \quad 0.95981 E-03 \quad 0.92187 E-03$

$0.79237 E-03 \quad 0.76460 E-03 \quad 0.73840 E-03$

0.64670 E-03 $0.62664 E-03 \quad 0.60752 E-03$

$0.53567 E-03 \quad 0.52463 E-03 \quad 0.51016 E-03$

$0.45819 E-03$

$\begin{array}{llll}0.71694 E-02 & 0.58189 E-02 & 0.48896 E-02\end{array}$

$0.29586 E-02 \quad 0.26868 E-02 \quad 0.24587 E-02$

$0.18214 E-02 \quad 0.17073 E-02 \quad 0.16054 E-02$

$0.12879 E-02 \quad 0.12252 E-02 \quad 0.11676 E-02$

$0.97751 E-03 \quad 0.93800 E-03 \quad 0.90114 E-03$

$\begin{array}{llll}0.77507 E-03 & 0.74807 E-03 & 0.72254 E-03\end{array}$

$0.63328 E-03 \quad 0.61370 E-03 \quad 0.59520 E-03$

$0.52505 E-03 \quad 0.51432 E-03 \quad 0.50029$ E-03.

$\begin{array}{lll}0.69914 E-02 & 0.56767 E-02 & 0.47718 E-02\end{array}$

$0.28876 E-02 \quad 0.26224 E-02 \quad 0.23996 E-02$

0.17781 E-02 $0.16668 E-02 \quad 0.15676 E-02$

$0.12580 E-02 \quad 0.11970 E-02 \quad 0.11409 E-02$

$0.95568 E-03 \quad 0.91726 E-03 \quad 0.88133 E-03$ 
$0.84769 E-03$ $0.68384 E-03$

$0.56600 E-03$

$0.47759 E-03$

$0.20904 E-01$

$0.40141 E-02$

$0.21584 \mathrm{E}-02$

$0.14446 \mathrm{E}-02$

$0.10650 E-02$

$0.82962 E-03$

$0.66987 . E-0.3$

$0.55492 E-03$
$0.46866 E-03$

$0.20288 E-01$
$0.39226 E-02$

$0.39226 E-02$
$0.21096 E-02$

$0.14123 E-02$

$0.10420 E-02$

$0.81234 \mathrm{E}-03$

$0.65652 E-03$

$0.54438 E-03$

0.19707 E-01

$0.38354 \mathrm{E}-02$

$0.20630 E-02$

$0.13817 \mathrm{E}-02$

$0.10199 E-02$

$0.79582 \mathrm{E}-03$

$0.64367 E-03$

$0.53418 E-03$

0.19158 E-O1

$0.37524 E-02$

$0.20187 E-02$
$0.81613 E-03$

$0.66154 E-03$

$0.54947 E-03 \quad 0.53381 E-03$

$.46494 \mathrm{E}-03$

$1=32$

$0.12486 E-01 \quad 0.88422 E-02 \quad 0.68220 E-02$

$0.35219 E-02 \quad 0.31339 E-02 \quad 0.28203 E-02$

$0.15987 E-02 \quad 0.18594 E-02 \quad 0.17369 E-02$

$0.13660 E-02 \quad 0.12947 E-02 \quad 0.12296 E-02$

$0.10184 E-02 \quad 0.97510 E-03 \quad 0.93492 E-03$

$0.79886 E-03 \quad 0.76997 E-03 \quad 0.74274 E-03$

$0.64818 E-03 \quad 0.62756 E-03 \quad 0.60811 E-03$

$0.53891 E-03 \quad 0.52356 E-03 \quad 0.50896 E-03$

$1=$

33

$0.12161 E-01 \quad 0.86261 E-02 \quad 0.66616 E-02$

$0.34418 E-02 \quad 0.30628 E-02 \quad 0.27562 E-02$

$0.19534 \mathrm{E}-02 \quad 0.18174 \mathrm{E}-02$

$0.13357 E-02 \quad 0.12660 E-02$

$0.99644 E-03 \quad 0.95429 E-03$

$0.78236 E-03 \quad 0.75415 E-03$

$0.63531 E-03 \quad 0.61532 E-03$

$0.52868 E-030.51376 E-03$ $\mathrm{I}=$ 34

$\begin{array}{lll}0.11853 E-01 & 0.84214 E-02 & 0.65 C 82 E-02 \\ 0.32656 E-02 & 0.29949 E-02 & 0.26953 E-02\end{array}$ $0.32656 \mathrm{E}-02 \quad 0.29949 \mathrm{E}-02$

$0.19104 E-02$

$0.17775 \mathrm{E}-02$

$0.13067 E-02 \quad 0.12388 \mathrm{E}-02$

$0.97555 E-03 \quad 0.93437 E-03$

$0.76653 \mathrm{E}-03 \quad 0.73897 \mathrm{E}-03$

$0.62309 E-03 \quad 0.60336 E-03$

$0.51891 E-03 \quad 0.50424 E-03$ $\mathrm{I}=$ 35

$0.11561 E-01 \quad 0.82258 E-02$

$0.32927 E-02 \quad 0.29304 E-02$

$0.18695 E-02$

$0.17394 E-02$
$0.73223 E-03 \quad 0.70734 E-03$

$0.60141 E-03 \quad 0.58318 E-03$

$0.50447 E-03 \quad 0.49069 E-03$

$0.55423 E-02 \quad 0.46595 E-02$

$0.25612 E-02 \quad 0.23437 E-02$

$0.16285 E-02 \quad 0.15315 E-02$

$0.11701 E-02 \quad 0.11154 E-02$

$0.89744 E-03 \quad 0.86241 E-03$

$0.71705 E-03 \quad 0.69283 E-03$

$0.58942 E-03 \quad 0.57183 E-03$

$0.49490 E-03 \quad 0.48155 E-03$

$0.54140 E-02 \quad 0.45526 E-02$

$0.25031 E-020.22906 E-02$

$0.15918 E-02 \quad 0.14973 E-02$

$\begin{array}{llll}0.12026 E-02 & 0.11445 E-02 & 0.10912 E-02 \\ 0.91506 E-03 & 0.87849 E-03 & 0.84433 E-03\end{array}$

$0.72757 E-03 \quad 0.70255 E-03 \quad 0.67887 E-03$

$0.59614 E-03 \quad 0.57810 E-03 \quad 0.56077 E-03$

$0.49941 E-03 \quad 0.48579 E-03 \quad 0.47265 E-03$

$0.16606 \mathrm{E}-02$

$0.11768 \mathrm{E}-02$

$0.89607 \mathrm{E}-03$

$0.71307 \mathrm{E}-03$

$0.58483 E-03$

$0.49032 \mathrm{E}-03$

$0.63619 \mathrm{E}-02$

$0.26 \geq 72 E-02$

$0.16252 \mathrm{E}-02$

$0.52916 E-02 \quad 0.44509 E-02$

$0.24478 E-02 \quad 0.22401 E-02$

$0.15571 E-02 \quad 0.14646 E-02$

$0.11201 E-020.10680 E-02$

$0.86038 E-03 \quad 0.82703 E-03$

$0.68860 E-03 \quad 0.66553 E-03$

$0.56706 E-03 \quad 0.55026 E-03$

$0.47691 E-03$

$0.51750 E-02 \quad 0.43538 E-02$

$0.23952 E-02 \quad 0.21919 E-02$

$0.15239 E-020.14335 E-02$ 


\begin{tabular}{|c|c|c|c|c|c|}
\hline $\begin{array}{l}.13524 E-02 \\
.99893 E-03 \\
.77997 E-03 \\
.63145 E-03 \\
.52442 E-03\end{array}$ & $\begin{array}{l}0.12792 E-02 \\
0.95555 E-03 \\
0.75133 E-03 \\
0.61113 E-03 \\
0.5 C 940 E-03\end{array}$ & $\begin{array}{l}0.12128 E-02 \\
0.91531 E-03 \\
0.72446 E-03 \\
0.59207 E-03 \\
0.49516 E-03\end{array}$ & $\begin{array}{l}0.11522 E-02 \\
0.87790 E-03 \\
0.69912 E-03 \\
0.57380 E-03 \\
0.48145 E-03\end{array}$ & $\begin{array}{l}0.10968 E-02 \\
0.84303 E-03 \\
0.67526 E-03 \\
0.55655 E-03\end{array}$ & $\begin{array}{l}0.10458 E-02 \\
0.81048 E-03 \\
0.65268 E-03 \\
0.54006 E-03\end{array}$ \\
\hline $\begin{array}{l}0.18641 E-01 \\
0.36728 E-02 \\
0.19765 E-02 \\
0.13245 E-02 \\
0.97881 E-03 \\
0.76475 E-03 \\
0.61950 E-03 \\
0.51492 E-03\end{array}$ & $\begin{array}{l}0.11283 E-01 \\
0.32234 E-02 \\
0.18303 E-02 \\
0.12529 E-02 \\
0.93640 E-03 \\
0.73681 E-03 \\
0.59985 E-03 \\
0.5 C 033 E-03 \\
I=\end{array}$ & $\begin{array}{l}0.80390 E-02 \\
0.28687 E-02 \\
0.17031 E-02 \\
0.11879 E-02 \\
0.89706 E-03 \\
0.71049 E-03 \\
0.58105 E-03 \\
0.48631 E-03\end{array}$ & $\begin{array}{l}E-02 \\
E-02 \\
E-02 \\
E-02 \\
E-03 \\
E-03 \\
E-03\end{array}$ & $\begin{array}{l}0.50636 \\
0.23449 \\
0.14922 \\
0.10744 \\
0.82644 \\
0.66240\end{array}$ & $\begin{array}{l}E-02 \\
B E-02 \\
E-02 \\
E-02 \\
E-03 \\
E-03 \\
E-03\end{array}$ \\
\hline $\begin{array}{l}0.18149 E-01 \\
0.35969 E-02 \\
0.19360 E-02 \\
0.12978 E-02 \\
0.95954 E-03 \\
0.75019 E-03 \\
0.60821 E-03 \\
0.50586 E-03\end{array}$ & $\begin{array}{l}0.11017 E-01 \\
0.31569 E-02 \\
0.17930 E-02 \\
0.12277 E-02 \\
0.91805 E-03 \\
0.72281 E-03 \\
0.58882 E=03 \\
0.49149 E-03 \\
1=38\end{array}$ & $\begin{array}{l}0.78609 E-02 \\
0.28099 E-02 \\
0.16684 E-02 \\
0.11641 E-02 \\
0.87958 E-03 \\
0.69712 E-03 \\
0.57055 E-03\end{array}$ & $\begin{array}{l}9 E-02 \\
8 E-02 \\
0 E-02 \\
1 E-02 \\
3 E-03 \\
0 E-03 \\
3 E-03\end{array}$ & $\begin{array}{l}E-02 \\
E-02 \\
E-02 \\
E-02 \\
E-03 \\
E-03 \\
-03\end{array}$ & $\begin{array}{l}02 \\
02 \\
02 \\
03 \\
03 \\
03\end{array}$ \\
\hline $\begin{array}{l}0.17683 E-01 \\
0.35242 E-02 \\
0.18974 E-02 \\
0.12722 E-02 \\
0.94106 E-03 \\
0.73615 E-03 \\
0.59718 E-03 \\
0.49703 E-03\end{array}$ & $\begin{array}{l}0.10764 E-01 \\
0.30935 E-02 \\
0.17573 E-02 \\
0.12035 E-02 \\
0.90045 E-03 \\
0.7 C 941 E-03 \\
0.57833 E-03\end{array}$ & $\begin{array}{l}0.76905 E-02 \\
0.27534 E-02 \\
0.16353 E-02 \\
0.11412 E-02 \\
0.86283 E-03 \\
0.68423 E-03 \\
0.56037 E-03\end{array}$ & $\begin{array}{l}0.59613 E-02 \\
0.24781 E-02 \\
0.15280 E-02 \\
0.10846 E-02 \\
0.82782 E-03 \\
C .66 C 65 E-03 \\
0.54339 E-03\end{array}$ & $\begin{array}{l}0.48548 E-02 \\
0.22510 E-02 \\
0.14330 E-02 \\
0.10327 E-02 \\
0.79516 E-03 \\
0.63820 E-03 \\
0.52714 E-03\end{array}$ & $\begin{array}{l}75 E-02 \\
00 E-02 \\
83 E-02 \\
99 E-03 \\
71 E-03 \\
20 E-03 \\
78 E-03\end{array}$ \\
\hline $0.34546 E-02$ & $\begin{array}{c}I=\quad 39 \\
0.10523 E-01 \\
0.30326 E-02 \\
0.17231 E-02\end{array}$ & $\begin{array}{l}0.75276 E-02 \\
0.26993 E-02 \\
0.16034 E-02\end{array}$ & $\begin{array}{l}0.58386 E-02 \\
0.24297 E-02 \\
0.14984 E-02\end{array}$ & $\begin{array}{l}0.47571 E-02 \\
0.22069 E-02 \\
0.14053 E-02\end{array}$ & $\begin{array}{l}0.40060 E-02 \\
0.20198 E-02 \\
0.13222 E-02\end{array}$ \\
\hline
\end{tabular}


$0.12477 E-02$ $0.92334 E-03$ $0.72271 E-03$ $0.58668 E-03$

$0.16820 E-01$ $0.33877 \mathrm{E}-02$

$0.18250 E-02$

$0.12242 E-02$

$0.90634 E-03$

$0.70972 E-03$

$0.57648 E-03$

$0.16420 E-01$

$0.33234 \mathrm{E}-02$

$0.17909 E-02$

$0.12017 E-02$

$0.88997 E-03$

$0.69737 E-03$

$0.56672 E-03$

$0.16037 E-01$

0.32620 E-02

$0.17583 E-02$

0.11800 E-02

$0.87420 E-03$

$0.68523 E-03$

$0.55718 E-03$

$0.15673 E-01$

$0.32025 E-02$

$0.17269 E-02$

$0.11591 E-02$

$0.85907 E-03$

$0.67375 E-03$

$0.54809 E-03$

$0.15996 E-02$ $0.88 .359 E-03$ $0.69647 E-03$ $0.56814 E-03$ $I=$

$0.11195 E-02$ $0.84672 E-03$ $0.67196 E-03$ $0.55064 E-03$ 40

$0.1 C 292 E-01$

$0.29741 E-02$

$0.16903 E-02$

$0.11583 E-02$

$0.86737 E-03$

$0.68417 \mathrm{E}-03$

$0.55840 E-03$ $I=$

0.7371 OE-02

$0.26477 E-02$

$0.15730 E-02$

$0.10985 E-02$

$0.83121 E-03$

$0.65992 E-03$

$0.54114 E-03$ 41

$0.10071 E-01$

$0.29182 E-02$

$0.16588 E-02$

$0.11370 E-02$

$0.85174 E-03$

$0.67209 E-03$

$0.54888 E-03$ $I=$

$0.72213 E-0.2$

$0.25978 E-02$

$0.15438 E-02$

$0.10784 \mathrm{E}-02$

$0.81635 E-03$

$0.64855 E-03$

$0.53208 E-03$ 42

$0.98594 E-02$

$0.28642 E-02$

$0.16286 E-02$

$0.11166 E-02$

$0.70774 E-02$

$0.25501 E-02$

$0.15157 \mathrm{E}-02$

$0.10590 E-02$

$0.83676 E-03$

$0.80200 E-03$

$0.63741 E-03$

$0.53981 E-03 \quad 0.52322 E-03$

$1=$ 43

$0.96564 E-0.2$

$0.28125 E-02$

$0.1096 \circ E-02$

$0.82228 E-03$

$0.64948 E-03$

$0.69395 E-02$

$0.25041 E-02$

$0.14887 \mathrm{E}-02$

$0.10404 E-02$

$0.78823 E-03$

$0.62680 E-03$

$0.53094 E-03$
$0.10639 E-02 \quad 0.10130 E-02 \quad 0.96636 E-03$

$0.81241 E-03 \quad 0.78049 E-03 \quad 0.75062 E-03$

$0.64865 E-03 \quad 0.62689 E-03 \quad 0.60616 E-03$

$0.53390 E-03 \quad 0.51809 E-0.3 \quad 0.50294 E-03$

$0.57213 E-02$

$0.23831 E-02$

$0.14700 E-02$

$0.10440 E-02$

$0.79765 E-03$

$0.63731 E-03$

$0.52485 E-03$

$0.46632 E-02 \quad 0.39281 E-02$

$0.21647 E-02 \quad 0.19812 E-02$

$0.13787 E-020.12972 E-02$

$0.99422 E-03 \quad 0.94847 E-03$

$0.76633 E-03 \quad 0.73712 E-03$

$0.61582 E-03 \quad 0.59564 E-03$

$0.50926 E-03$

C. $56085 E-02$

$0.23385 E-02$

$0.14427 E-02$

$0.10250 E-02$

$0.78341 E-03$

$0.62622 E-03$

$0.45733 E-02 \quad 0.38530 E-02$

$0.21242 E-020.19443 E-02$

$0.13531 E-02 \quad 0.12732 E-02$

$0.97613 E-03 \quad 0.93130 E-03$

$0.75275 E-03 \quad 0.72407 E-03$

$0.60528 E-03 \quad 0.58542 E-03$

$0.51601 \mathrm{E}-0.3$

$0.55 C 05 E-02$

$0.22956 E-02$

$0.14165 E-02$

$0.44866 E-02 \quad 0.37808 E-02$

$0.20854 E-02 \quad 0.19087 E-02$

$0.13286 E-02 \quad 0.12503 E-02$

$0.10 C 67 E-02 \quad 0.95877 E-03 \quad 0.91477 E-03$

$0.76974 E-03 \quad 0.73963 E-03 \quad 0.71165 E-03$

$0.61564 E-03 \quad 0.59503 E-03 \quad 0.57564 E-03$

C. $53962 \mathrm{E}-02$

$0.22544 E-02$

$0.13913 \mathrm{E}-02$

$0.98906 E-03$

$0.75653 E-03$

$0.60536 \mathrm{E}-03$

$0.44031 E-02 \quad 0.37117 E-02$

$0.20479 E-02 \quad 0.18746 E-02$

$0.13051 E-02 \quad 0.12281 E-02$

$0.94203 E-03 \quad 0.89882 E-03$

$0.72714 \mathrm{E}-03 \quad 0.69945 \mathrm{E}-03$

$0.58522 E-03 \quad 0.56608 E-03$ 


$$
I=44
$$

$0.15325 E-01$ $0.31455 E-02$ $0.16967 E-02$ $0.11391 \mathrm{E}-02$ $0.84444 E-03$ $0.66251 E-03$ $0.53920 \mathrm{E}-03$

$0.14992 \mathrm{E}-01$ $0.30904 E-02$ $0.16675 E-02$ $0.11198 E-02$ $0.83039 E-03$

$0.65178 E-03$

$0.14673 E-01$

$0.30374 E-02$

$0.16394 E-02$

$0.11012 \mathrm{E}-02$

$0.81678 E-03$

$0.64133 E-03$

0.14367 E-01

$0.29861 E-02$

$0.16124 E-02$

$0.10832 E-02$

$0.80381 E-03$

$0.63132 E-03$

$0.14075 E-01$

$0.29367 E-02$

$0.15863 E-02$

$0.10658 E-02$

$0.79 .100 \mathrm{E}-03$

$0.62151 E-03$
$0.94622 E-02$

0.68065 E-02

$\begin{array}{ll}7626 E-02 & 0.24599 E-02\end{array}$

$0.15716 E-02 \quad 0.14627 E-02$

$0.10780 E-02 \quad 0.10226 E-02$

$0.80838 E-03 \quad 0.77490 E-0.3$

$0.63882 E-03 \quad 0.61647 E-03$

$\mathrm{I}=$ 45 .

$0.92750 E-02 \quad 0.66786 E-02$

$0.27146 E-02 \quad 0.24173 E-02$

$0.15446 E-02 \quad 0.14377 E-02$

0.1 C598E-02 $0.10053 E-02$

$\begin{array}{lll}0.79 .493 E-03 & 0.76221 E-03\end{array}$

$0.62843 E-03 \quad 0.60657 E-03$ $I=$ 46

0.9 C952E-02 0.65560 E-02

$0.26682 E-02 \quad 0.23762 E-02$

$0.15188 E-02 \quad 0.14136 E-02$

$0.1 C 422 E-02 \quad 0.98864 E-03$

$0.78210 E-03 \quad 0.74970 E-03$

$0.61849 E-03 \quad 0.59689 E-03$ $I=$ 47

$0.89230 E-02 \quad 0.64372 E-02$

$0.26235 E-02 \quad 0.23366 E-02$

$0.14937 E-02 \quad 0.13904 E-02$

$0.10252 E-02 \quad 0.97262 E-03$

$0.76945 E-03 \quad 0.73786 E-03$

$0.6 C 875 E-03 \quad 0.58765 E-03$ $1=$ 48

$0.87564 E-02 \quad 0.63230 E \div 02$

$0.25804 E-02 \quad 0.22984 E-02$

$0.14696 \mathrm{E}-02 \quad 0.13679 \mathrm{E}-02$

$0.1 C 088 E-02 \quad 0.95709 E-03$

$0.75749 E-03$

$0.59 \$ 45 E-03$

$0.72623 E-03$
$0.57859 E-03$
$0.52959 E-02 \quad 0.43233 E-02 \quad 0.36448 E-02$ $0.22146 E-02 \quad 0.20120 E-02 \quad 0.18417 E-02$ $0.13671 E-02 \quad 0.12824 E-02 \quad 0.12069 E-02$ $0.97208 E-03 \quad 0.92588 E-03 \quad 0.88352 E-03$ $0.74394 E-03 \quad 0.71484 E-03 \quad 0.68790 E-0.3$ $0.59551 E-03 \quad 0.57563 E-0.3 \quad 0.55696 E-03$

$0.51998 E-02 \quad 0.42458 E-02 \quad 0.35806 E-02$ $0.21764 E-02 \quad 0.19774 E-02 \quad 0.18101 E-02$ $0.13438 \mathrm{E}-02 \quad 0.12605 \mathrm{E}-02 \quad 0.11863 \mathrm{E}-02$ $0.95569 E-03 \quad 0.91038 E-03 \quad 0.86872 E-03$ $\begin{array}{lll}0.73155 E-03 & 0.70322 E-03 & 0.67658 E-03\end{array}$ $0.58588 E-03 \quad 0.56648 E-03 \quad 0.54804 E-03$ $0.51066 \mathrm{E}-02 \quad 0.41715 \mathrm{E}-02 \quad 0.35186 \mathrm{E}-02$ $0.21396 E-02 \quad 0.19440 E-02 \quad 0.17795 E-02$ $0.13213 E-02 \quad 0.12395 E-02 \quad 0.11666 E-02$ $0.93995 E-03 \quad 0.89536 E-03 \quad 0.85449 E-03$ $0.71983 E-03 \quad 0.69181 E-03 \quad 0.66578 E-03$ $0.57669 E-03 \quad 0.55752 E-03$

C.50171E-02 $0.40996 E-02 \quad 0.34588 E-02$ 0.21040 E-02 $0.19117 E-02 \quad 0.17500 E-02$ $0.12996 E-02 \quad 0.12192 E-02 \quad 0.11475 E-02$ $0.92470 E-03 \quad 0.88093 E-03 \quad 0.84070 E-03$ $0.70832 E-03 \quad 0.68093 E-03 \quad 0.65526 E-03$ $0.56768 E-03$

0.49306 E-02 $0.40303 E-02 \quad 0.34009 E-02$ $0.20697 E-02 \quad 0.18805 E-02 \quad 0.17217 E-02$ $0.12786 E-02 \quad 0.11996 E-02 \quad 0.11291 E-02$ $0.91003 E-03 \quad 0.86693 E-03 \quad 0.82755 E-03$ $\begin{array}{llll}0.69733 E-03 & 0.67031 E-03 & 0.64517 E-03\end{array}$ 
$I=49$

0.13793 E-01

$0.28890 E-02$

$0.15611 \mathrm{E}-02$

$0.10491 E-02$

$0.77889 E-03$

$0.61214 E-03$

$0.13523 E-01$

$0.28428 E-02$

$0.15367 E-02$

$0.10329 E-02$

$0.76696 E-03$

$0.60295 E-03$

$0.60295 E-03$

$0.13263 E-01$

$0.27980 E-02$

0.15131 E-02

$0.10173 E-02$

$0.75557 E-03$

$0.13014 E-01$

0.27547 E-02

$0.14903 E-02$

$0.10021 E-02$

$0.7 .4444 \mathrm{E}-03$

$0.12772 E-01$

$0.27129 E-02$

$0.14682 \mathrm{E}-02$

$0.98752 E-03$

$0.73375 E-03$

$0.12541 \mathrm{E}-01$

$0.26 .723 E-02$

$0.14467 \mathrm{E}-02$

$$
1=50
$$

$$
I=51
$$

$1=$ 52

$1=$ 53

$1=$ 54
$0.85964 E-02$

$0.25387 E-02$

$0.14463 E-02$

$0.99297 E-03$

$0.74572 E-03$

$0.59033 E-03$

$0.84419 E-02 \quad 0.61063 E-02 \quad 0.47663 E-02$

$0.24983 E-02 \quad 0.22256 E-02 \quad 0.20045 E-02$

$0.14237 E-02 \quad 0.13254 E-02 \quad 0.12389 E-02$

$\begin{array}{lll}0.97771 E-03 & 0.92763 E-03 & 0.88220 E-03\end{array}$

$0.73448 E-03 \quad 0.70429 E-03 \quad 0.67633 E-03$

$0.82931 E-02 \quad 0.60033 E-02$

$0.24592 E-02 \quad 0.21911 E-02$

$0.14019 E-02 \quad 0.13051 E-02$

$0.96288 E-03 \quad 0.91377 E-03$

$0.72351 E-03 \quad 0.69390 E-03$

$0.81492 E-02 \quad 0.59040 E-02$

$0.24216 E-02 \quad 0.21576 E-02$

$0.13808 E-02 \quad 0.12854 E-02$

$0.94871 E-03 \quad 0.90005 E-03$

$0.71298 E-03 \quad 0.68369 E-03$

$0.80104 E-02 \quad 0.58078 E-02$

$0.23850 E-02 \quad 0.21252 E-02$

$0.13603 E-02 \quad 0.12665 E-02$

$0.93467 E-03 \quad 0.88706 E-03$

$0.70263 E-03 \quad 0.67393 E-03$

$0.78763 E-02 \quad 0.57148 E-02$

$0.23495 E-02 \quad 0.20937 E-02$

$0.13406 E-02$
$0.12480 E-02$
$0.48471 E-02$

$0.20364 E-02$

$0.68661 \mathrm{E}-03$

$0.46883 \dot{E}-02$

$0.19734 E-02$

$0.12200 \mathrm{E}-02$

$0.66625 E-03$

$0.46128 \mathrm{E}-02$

$0.19434 \mathrm{E}-02$

$0.12017 \mathrm{E}-02$

$0.85604 E-03$

$0.65660 E-03$

$0.45397 \mathrm{E}-02$

$0.19143 E-02$

C. $11839 \mathrm{E}-02$

$0.843 .48 E-03$

$0.64713 E-03$

$0.44688 E-02$

$0.18861 \mathrm{E}-02$

$0.11668 \mathrm{E}-02$
$0.12585 \mathrm{E}-02$

$0.86876 E-03$
$0.39632 E-02 \quad 0.33451 E-02$

$0.18506 E-02 \quad 0.16943 E-02$

$0.11807 E-02 \quad 0.11113 E-02$

$0.85358 E-030.81456 E-03$

$0.66014 E-03 \quad 0.63528 E-03$

$0.38985 E-02 \quad 0.32912 E-02$

$0.18215 E-020.16677 E-02$

$0.11623 E-02 \quad 0.10941 E-02$

$0.84038 E-03 \quad 0.80227 E-03$

$0.65015 E-03 \quad 0.62583 E-03$

$0.38359 E-02 \quad 0.32390 E-02$

$0.17934 \mathrm{E}-02 \quad 0.16421 \mathrm{E}-02$

$0.11447 E-020.10775 E-02$

$0.82789 E-030.79016 E-03$

$0.64062 E-030.61656 E-03$

$0.37752 E-02 \quad 0.31884 E-02$

$0.17662 E-02 \quad 0.16172 E-02$

$0.11275 E-02 \quad 0.10614 . E-02$

$0.81557 E-030.77859 E-03$

$0.63125 E-03$

$0.37164 E-02 \quad 0.31393 E-02$

$0.17399 E-02 \quad 0.15932 E-0.2$

$0.11109 E-02 \quad 0.10458 E-02$

$0.80380 E-03 \quad 0.76728 E-03$

$0.36594 E-02 \quad 0.30920 E-02$

$0.17144 E-02 \quad 0.15699 E-02$

$0.10948 E-02 \quad 0.10308 E-02$ 
$0.97312 E-03 \quad 0.92138 E-03 \quad 0.87424 E-03$

$0.72324 E-03 \quad 0.69273 E-03 \quad 0.66433 E-03$

$$
\mathrm{I}=
$$

$0.12317 E-01$

$0.26329 E-02$

$0.14260 E-02$

$0.95948 E-03$

$0.71320 E-03$

$0.12101 E-01$

$0.25947 E-02$

$0.14058 \mathrm{E}-02$

$0.94600 E-03$

$0.70331 E-03$

$0.77466 E-02 \quad 0.56245 E-02$

$0.23151 E-02 \quad 0.20633 E-02$

$0.13213 E-02 \quad 0.12302 E-02$

$0.9 C 824 E-03 \quad 0.86198 E-03$

$0.68300 E-03$

$$
1=
$$$$
56
$$

$0.76209 E-02 \quad 0.55370 E-02$

$0.22818 E-020.20338 E-02$

$0.13027 E-02 \quad 0.12129 E-02$

$0.89569 E-03 \quad 0.84997 E-03$

$$
I=57
$$

$0.11892 E-01$ $0.25576 E-02$

$0.13863 E-02$

$0.93309 E-03$

$0.74992 E-02$

$0.22495 E-02$

$0.12846 E-02$

$0.88338 E-03$ $1=$ 58

$0.11691 E-01$ $0.25217 E-02$ $0.13672 \mathrm{E}-02$ $0.92044 E-03$

$0.73819 E-02$

$0.22180 E-02$

$0.12673 E-02$

$0.87154 E-03$ $1=$ 59

$0.11497 E-01$ $0.24866 \mathrm{E}-02$ $0.13490 E-02$ $0.90827 E-03$

$0.12679 E-02$
$0.21874 E-02$

$0.12500 E-02$

$0.85986 E-03$ $1=$ 60

$0.11309 E-01 \quad 0.71574 E-02 \quad 0.52137 E-02$ $0.24525 \mathrm{E}-02$ $0.13308 E-02$ $0.89626 E-03$

$0.21577 E-02 \quad 0.19237 E-02$

$0.12336 E-02 \quad 0.11486 E-02$

$0.84869 E-03 \quad 0.80541 E-03$ $I=61$

$0.11127 E-01 \quad 0.70501 E-02 \quad 0.51386 E-02$

$0.54527 E-02$
$0.83149 E-03 \quad 0.79229 E-03 \quad 0.75642 E-03$

$0.44001 E-02 \quad 0.36044 E-02 \quad 0.30460 E-02$

$0.18589 E-02 \quad 0.16896 E-02 \quad 0.15473 E-02$

$0.11501 E-02 \quad 0.10794 E-02 \quad 0.10160 E-02$

$0.81974 E-0.3 \quad 0.78123 E-03 \quad 0.74574 E-03$

$0.43338 E-02 \quad 0.35509 E-02 \quad 0.30014 E-02$

$0.18323 E-02 \quad 0.16656 E-02 \quad 0.15254 E-02$

$0.11342 E-02 \quad 0.10641 E-02 \quad 0.10020 E-02$

$0.80845 E-03 \quad 0.77034 E-03 \quad 0.73552 E-03$

$0.42693 E-02$

$0.34990 E-02 \quad 0.29580 E-02$

$0.18065 E-02 \quad 0.16423 E-02 \quad 0.15041 E-02$

$0.11183 E-02 \quad 0.10496 E-02 \quad 0.98809 E-03$

$0.79734 E-03 \quad 0.75993 E-03 \quad 0.72545 E-03$

$0.42 C 67 E-02 \quad 0.34486 E-02 \quad 0.29160 E-02$

$0.17816 \mathrm{E}-02 \quad 0.16196 \mathrm{E}-02 \quad 0.14834 \mathrm{E}-02$

$\begin{array}{llll}0.11033 E-02 & 0.10352 E-02 & 0.97479 E-03\end{array}$

$0.78670 E-03 \quad 0.74966 E-03$

\section{$0.41460 E-02 \quad 0.33997 E-02 \quad 0.28752 E-02$}

$0.17572 E-02 \quad 0.15977 E-02 \quad 0.14633 E-02$

$0.10884 E-02 \quad 0.10215 E-02 \quad 0.96175 E-03$

$0.77621 E-03$

$0.40869 E-02 \quad 0.33522 E-02 \quad 0.28355 E-02$

$0.17337 E-02 \quad 0.15762 E-02 \quad 0.14440 E-02$

C. 10741 E-02 $0.10080 E-02 \quad 0.94919 E-03$

1

0.40297 E-02 $0.33060 E-02 \quad 0.27968 E-02$. 
$0.24195 E-02$ $0.13136 E-02$

$0.88476 E-03$

$0.10950 E-01$

$0.23873 E-02$

$0.12965 E-02$

$0.87339 E-03$

0.10780 E-0.1

$0.23560 E-02$

$0.12801 E-02$

$0.10614 E-01$

$0.23255, E-02$

$0.12640 E-02$

$0.10453 E-01$

$0.22961 E-02$

$0.12484 \mathrm{E}-02$

$0.10297 E-01$ $0.22668 \mathrm{E}-02$

$0.12330 \mathrm{E}-02$

$0.10146 E-01$ $0.22390 E-02$

$0.12182 \mathrm{E}-02$

$0.99994 E-02$

$0.22112 E-02$

$0.12036 E-02$

0.98570 E-02 $0.21846 E-02$
$0.21288 E-02 \quad 0.18982$ E-02 0.17107 E-02

$0.12174 E-02 \quad 0.11337 E-02 \quad 0.10601 E-02$

$0.83765 E-03$

$$
I=62
$$

$0.69461 E-02 \quad 0.50659 E-02 \quad 0.39739 E-02$

$0.21007 E-02 \quad 0.18732 E-02 \quad 0.16886 E-02$

$0.12018 E-02$

$0.11191 \mathrm{E}-02$

$0.10466 E-02$ $I=$$$
I=63
$$

$0.68452 E-02 \quad 0.49950 E-02 \quad 0.39195 E-02$

$\begin{array}{lll}0.20733 E-02 & 0.18492 E-02 & 0.16666 E-02\end{array}$

0.118

$0.10333 E-02$ $1=$ 64

$0.67470 E-02 \quad 0.49259 E-02$

$0.20470 E-02 \quad 0.18253 E-02$

$0.11717 \mathrm{E}-02 \quad 0.10911 \mathrm{E}-02$ $I=65$

$0.38669 E-02$

$0.16457 \mathrm{E}-02$

$0.10205 E-02$

$0.66514 E-02 \quad 0.48590 E-02 \quad 0.38154 E-02$

$0.20207 E-02 \quad 0.18026 E-02 \quad 0.16249 E-02$

$0.11571 E-02 \quad 0.10778 E-02 \quad 0.10 C 78 E-02$ $I=66$

$0.65590 E-02 \quad 0.47936 E-02$

$0.19958 E-02$ 0.17800E-02

$0.11431 E-02 \quad 0.10645 E-02$ $I=67$

$0.64686 E-02 \quad 0.47303 E-02$

$0.19709 E-02 \quad 0.17583 E-02$

$0.11292 E-02$

$$
I=68
$$

$0.63812 E-02 \quad 0.46683 E-02$

$0.19471 E-02 \quad 0.17370 E-02$

$\begin{array}{ll}0.46683 E-02 & 0.36699 E-02 \\ 0.17370 E-02 & 0.15663 E-02\end{array}$

$0.37167 E-02$

C. $15853 \mathrm{E}-02$

$0.15557 E-02 \quad 0.14248 E-02$ $0.99501 E-03 \quad 0.93680 E-03$

$0.32609 E-02 \quad 0.27593 E-02$

$0.15352 E-02 \quad 0.14066 E-02$

$0.98217 E-03 \quad 0.92493 E-03$

$0.32173 E-02 \quad 0.27227 E-02$

$0.15157 E-02 \quad 0.13884 E-02$

$0.96988 E-030.91319 E-03$

$0.31746 E-02 \quad 0.26872 E-02$ $0.14964 E-02 \quad 0.13711 E-02$ $0.95771 E-03$

$0.31332 E-02 \quad 0.26524 E-02$ 0.14779 E-02 0.13540 E-02 $I=69$

$0.62956 E-02 \quad 0.46088 E-02$

$0.19235 E-02 \quad 0.17163 E-02$ $\mathrm{I}=$
$0.36229 E-02$

$0.15475 \mathrm{E}-02$
0

.30927E-02 0.26191 E-02

$0.14596 E-020.13375 E-02$

$0.30539 E-02 \quad 0.25858 E-02$ $0.14420 E-02 \quad 0.13211 E-02$

$0.30150 E-02 \quad 0.25541$ E-02 $0.1 .4245 E-02 \quad 0.13055 E-02$

$0.29781 E+02 \quad 0.25225 E-02$ $0.14078 E-02 \quad 0.12899 E-02$ 


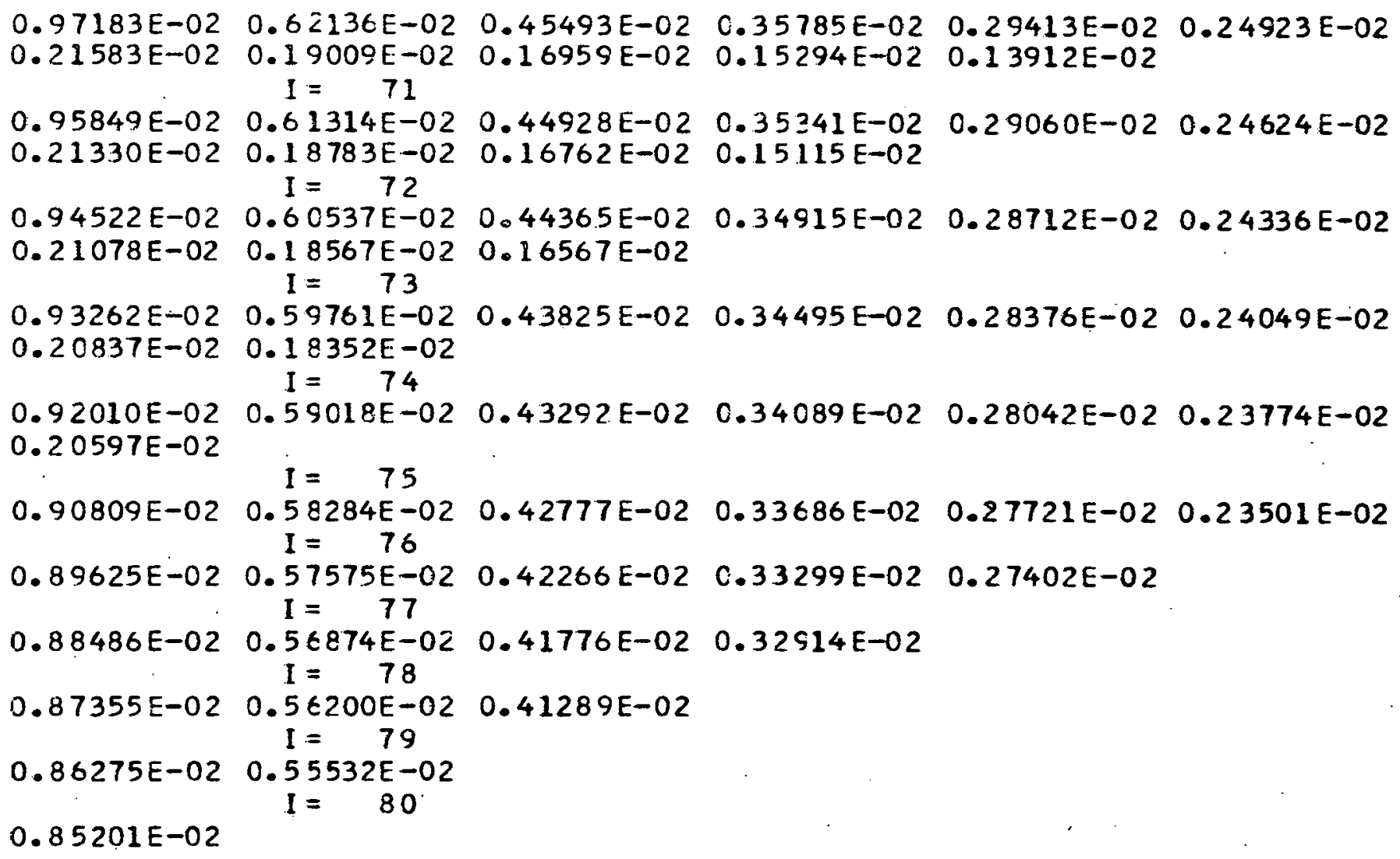


THE PHOTON FLUX FROM THE BLANKET NO.2

$\begin{array}{lllllllllllllll}0.84949 E & 05 & 0.63603 E & 05 & 0.54138 E & 05 & 0.61731 E & 05 & 0.61825 E & 05 & 0.71540 E & 05\end{array}$ $0.89881 E$ OS $0.74696 \mathrm{E}$ O5 $0.55368 \mathrm{E}$ 05 $0.38 E 23 \mathrm{E}$ O5 $0.48860 \mathrm{E}$ O5 $0.45496 \mathrm{E}$ OS $\begin{array}{lllllllllllll}0.42083 E & 05 & 0.4 C 914 E & 05 & 0.44068 E & 05 & 0.36788 E & 05 & 0.32835 E & 05 & 0.30216 E & 05\end{array}$

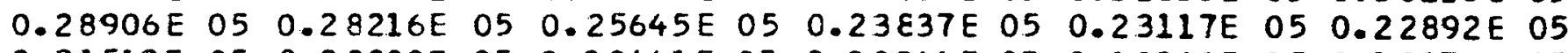
$0.21513 \mathrm{E}$ 05 $0.20080 \mathrm{E}$ O5 $0.20441 \mathrm{E}$ 05 $0.20246 \mathrm{E}$ O5 $0.18342 \mathrm{E} \quad 050.18270 \mathrm{E} \quad 05$ $\begin{array}{lllllllllllll}0.18487 E & 05 & 0.17296 E & 05 & 0.18398 E & 05 & 0.16922 E & 05 & 0.16486 E & 05 & 0.14975 E & 05\end{array}$

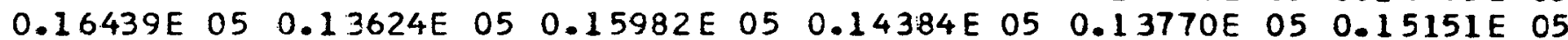

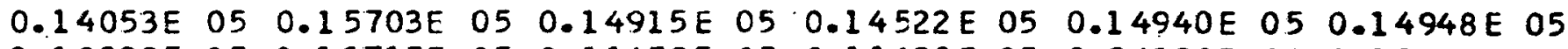
$\begin{array}{llllllllllllll}0.10992 E & 05 & 0.11715 E & 05 & 0.11452 E & 05 & C .11633 E & 05 & 0.94130 E & 04 & 0.13238 E & 05\end{array}$

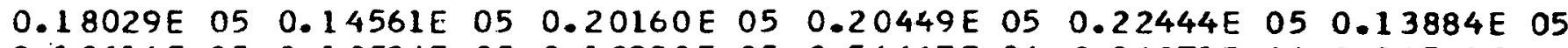

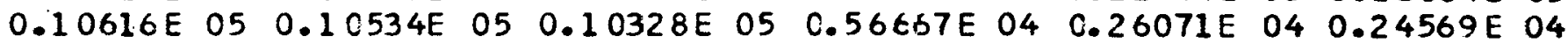
$\begin{array}{llllllllllllll}0.16557 E & 04 & 0.20989 E & 04 & 0.22571 E & 04 & 0.20321 E & 04 & 0.20974 E & 04 & 0.17191 E & 04\end{array}$ $\begin{array}{llllllllllllllll}0.17457 E & 04 & 0.13684 E & 04 & 0.96811 E & 03 & 0.65377 E & 03 & 0.85667 E & 03 & 0.28395 E & 03\end{array}$ 0.35928 E $03 \quad 0.50547 E \quad 03$ 
Appendix $\mathrm{H}$

INPUT DATA OF RUNS NO. 38, 39, and 40

These data were used to determine the moisture content in $\mathrm{Na}_{2} \mathrm{CrO}_{4}$. The irratiation facility was $4 \mathrm{TH}$ at the MITR. The descriptions of each run are in Table H-I. Immediately after the table the input data follows.

\section{TABLE $\cdot \mathrm{H}-\mathrm{I}$}

Descriptions of Runs No. 38, 39, and 40 : :

\begin{tabular}{|c|c|c|c|c|}
\hline \multicolumn{2}{|c|}{ Run, Number } & 38 & 39 & 40 \\
\hline \multicolumn{2}{|c|}{$\begin{array}{c}\text { Irradiation Time } \\
\text { (min.) }\end{array}$} & 468 & 463 & 468 \\
\hline \multirow{3}{*}{$\begin{array}{l}\text { Weight } \\
\text { of Samples } \\
(\mathrm{gm})\end{array}$} & $\dot{\mathrm{Na}}{ }_{2} \mathrm{CrO}_{4}$ & 12.3718 & 0 & 12.3718 \\
\hline & Mylar & 0.7710 & 0 & 0 \\
\hline & Al-can & 11.6660 & 11.4248 & 11.6660 \\
\hline \multicolumn{2}{|c|}{$\begin{array}{c}\text { Percent Loss Due } \\
\text { to Counting Dead } \\
\text { Time }\end{array}$} & 3.5 & $<1.0$ & 3.0 \\
\hline \multicolumn{2}{|c|}{$\begin{array}{l}\text { Diameter of } \\
\text { Collimators } \\
\text { (inch) }\end{array}$} & $3 / 4$ & $3 / 4$ & $3 / 4$ \\
\hline
\end{tabular}

Run numbers and line numbers are labeled at the end of printing lines. 


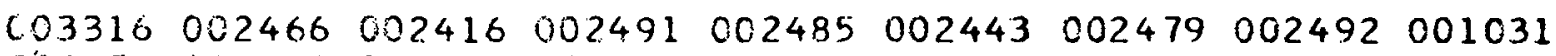
$002454 \quad 002429002347 \quad 002321002252 \quad 002354 \quad 002413 \quad 002417001039$

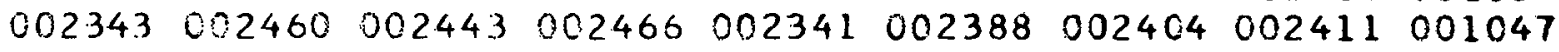
$0.02278 \quad 002460 \quad 002444 \quad 002417002305 \quad 002433 \quad 0023 C 1 \quad 002406 \quad 001055$

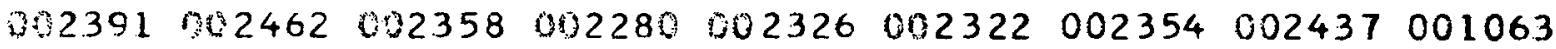
$002232002446 \quad 002372 \quad 002342 \quad 002343 \quad 002326 \quad 002345002425001071$ $\begin{array}{lllllllll}002467 & 002545 & 002659 & 002571 & 002617 & 002834 & 002868 & 002901 & 001079\end{array}$ $\begin{array}{lllllllll}002725 & 002725 & 002549 & 002380 & 002267 & 002310 & 002342 & 002312 & 001087\end{array}$ $\begin{array}{lllllllll}002323 & 002505 & 002476 & 002435 & 00245 C & 002450 & 002434 & 002281 & 001095\end{array}$ $022266 \quad 002447002247 \quad 002303 \quad 002310 \quad 002304 \quad 002166 \quad 002348001103$ $\begin{array}{lllllllll}002209 & 002354 & 002308 & 002324 & 002203 & 002219 & 002329 & 002290 & 001111\end{array}$ $002344002325002258 \quad 002266 \quad 002171 \quad 002278 \quad 002261002324001119$ $002296 \quad 002284 \quad 002276 \quad 002264 \quad 002268 \quad 002320 \quad 002214 \quad 002301001127$ $002133002222002251 \quad 002199002237 \quad 002197002267 \quad 002262001135$ $\begin{array}{llllllllll}0 & 02159 & 002188 & 002222 & 002191 & 002176 & 002158 & 002229 & 002272 & 001143\end{array}$ $002224 \quad 002289002117002143002198002203 \quad 002185002217001151$ $002226 \quad 002167002199002127 \quad 002107 \quad 002263 \quad 002167002300 \quad 001159$ $002119002197 \quad 002288 \quad 002251 \quad 002244 \quad 002313 \quad 002360 \quad 002449001167$ $002312002246 \quad 002249002189002147 \quad 002227 \quad 002184002172 \quad 001175$ $002127 \quad 002187 \quad 002237 \quad 002156 \quad 002021002095 \quad 002110002247001183$ 0) $12158 \quad 012137 \quad 002138 \quad 002107 \quad 002126 \quad 002175 \quad 002162002148001191$ 002012002179002185002123002011002117002094002145001199 $002127002165 \quad 002157002111002039002139002151002170001207$ $002069002122002110 \quad 002124001983 \quad 002134002028002161001215$ 002097002210002155002039002025002102002085002236001223 $002695 \quad 002136 \quad 002078 \quad 002104002122 \quad 002273 \quad 002202 \quad 002263001231$ $042227002177 \quad 002166 \quad 002016 \quad 001983 \quad 002158002007002147001239$ $002113002678 \quad 002061 \quad 002075 \quad 002065 \quad 002055 \quad 002085002123001247$ $602041 \quad 002063 \quad 002080 \quad 002103 \quad 002070 \quad 002192 \quad 002100 \quad 002092001255$ $001900 \quad 002051 \quad 002028001961001965 \quad 002062001972002117001263$ $001957032050 \quad 002443 \quad 001980 \quad 001877002023 \quad 002008002064001271$ 001972001964002043001935001937001931001938002050001279

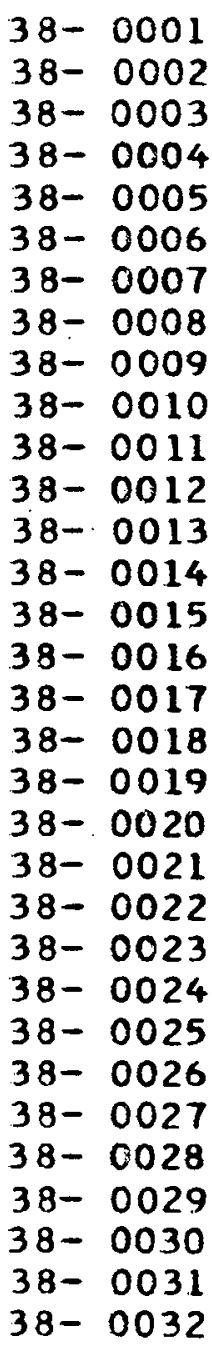


$000604 \quad 000585 \quad 000599 \quad 000541 \quad 000569000571000603 \quad 000597001031$ 000594000571000559000614000558000610001574000617001039

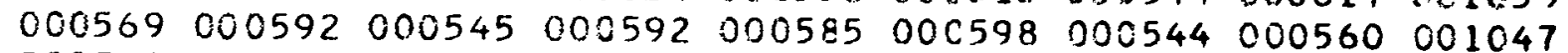

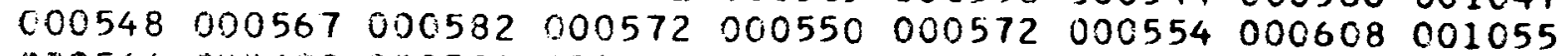
$000566 \quad 000602000581 \quad 000558 \quad 000519000550 \quad 000552000541001063$ $000549000581000590 \quad 000581000549000568 \quad 000561000604001071$ $000553 \quad 060596 \quad 000583 \quad 000533000592 \quad 000558 \quad 006604000591001079$ 000574000648000603000565000508000527000562000571001087 $\begin{array}{lllllllllll}0 & 00529 & 000568 & 000561 & 000521 & 000506 & 000537 & 000528 & 000523 & 001095\end{array}$

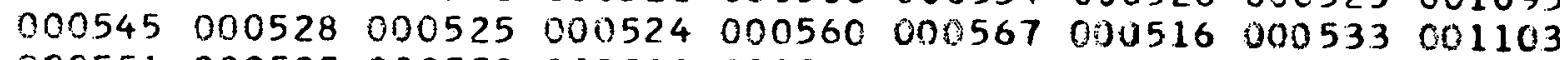

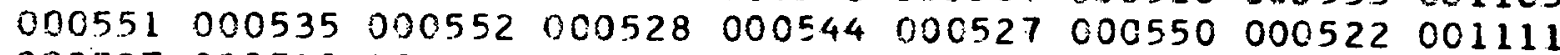
$000527000540 \quad 000559000530 \quad 000530000545000529000566001119$ 000556000555000546000547000559000535000548000568001127 $000554 \quad 000552 \quad 000552 \quad 000573 \quad 000544000522000565000520001135$ $00059200051200052700056900052400<478 \quad 00 G 499000550 \quad 001143$ $00486 \quad 00537000582 \quad 009530 \quad 000489000551000539000556001151$ $000505000520000498 \quad 000505000509000525 \quad 00503 \quad 000528001159$

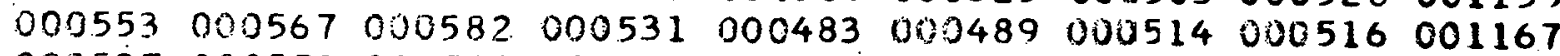
$\begin{array}{llllllllll}0 & 00527 & 000551 & 0005 C 8 & 000471 & 000473 & 000551 & 000522 & 000561 & 001175\end{array}$ 000492.000502000518000515000504000508006502000495001183 $300522000530 \quad 000502000506000517000491009529000474001191$ $\begin{array}{lllllllll}00513 & 000473 & 000473 & 000543 & 000495 & 000491 & 000523 & 000503 & 001199\end{array}$ $000463 \quad 000524 \quad 000520000465000515000493000505000520001207$ $000486000469000488 \quad 000494000462 \quad 000523000485000497001215$ $000505000519000512000528000460 \quad 000471000520000520001223$ $000504070520 \quad 000514000530 \quad 000403 \quad 000515000518000444001231$ $000500 \quad 000521000529000493 \quad 000464000514000469000503001239$ $000494000530 \quad 000464000500 \quad 000432 \quad 000483 \quad 000494000452001247$ $000468 \quad 000510 \quad 000510 \quad 000465 \quad 000445000472 \quad 003462 \quad 000483001255$ $000486 \quad 000497000470 \quad 000470 \quad 000465000469000468 \quad 000472001263$ $000508000477000515000467000477000470 \quad 000517000541001271$ $000470000493 \quad 000504000454 \quad 000471000525000483000469001279$

39- 0001

39- 0002

39- 0003

39- 0004

39- 0005

39- 0006

39- 0007

39- 0008

39- 0009

39-0010

39-0011

39- 0012

39- 0013

$39-0014$

39- 0015

39- 0016

39- 0017

39- 0018

39- 0019

39- 0020

39- 0021

39- 0022

39- 0023

39- 0024

39- 0025

39- 0026

39- 0027

$39-00.28$

39- 0029

$39-0030$

$39-0031$

39- 0032 
$001975 \quad 002066 \quad 001952 \quad 001988 \quad 001896 \quad 001886 \quad 001992001925 \quad 001031$ $\begin{array}{llllllllll}001876 & 001966 & 001876 & 001869 & 001789 & 001 \varepsilon 79 & 001820 & 001884 & 001039\end{array}$

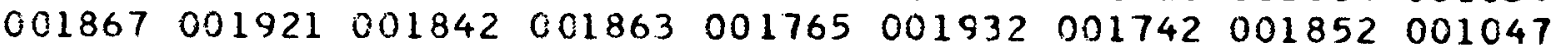
$001743 \quad 001812001883 \quad 001842 \quad 001797 \quad 001789001738 \quad 001818001055$ $\begin{array}{llllllllll}001726 & 001821 & 001773 & 001850 & 001701 & 001841 & 001790 & 001869 & 001063\end{array}$ $\begin{array}{llllllllll}0 & 01847 & 001838 & 001846 & 001770 & 001790 & 001940 & 001787 & 001845 & 001071\end{array}$ $001800 \quad 001830 \quad 001816 \quad 001845001806 \quad 001858 \quad 001997 \quad 001912001079$ $\begin{array}{lllllllllll}C 01908 & 001931 & 001879 & 001915 & 001834 & 001844 & 001794 & 001885 & 001087\end{array}$ $001748 \quad 011857 \quad 001920 \quad 001976 \quad 001868001860001856 \quad 001811001095$ $001685001784001678 \quad 001750 \quad 001654 \quad 001719001752 \quad 001789001103$ $\begin{array}{llllllllll}001763 & 001750 & 001718 & 001730 & 001671 & 001745 & 001752 & 001851 & 001111\end{array}$ $001734001787 \quad 001773 \quad 001779001719001778001673 \quad 001814001119$ $\begin{array}{llllllllll}0 & 01732 & 001814 & 001750 & 001785 & 001659 & 001691 & 001713 & 001758 & 001127\end{array}$ $001693 \quad 001747 \quad 001729001663 \quad 001615001709001616 \quad 001760 \quad 001135$ $001692001698001648 \quad 001669001666001719001719001689001143$ $001675001679001697 \quad 001741 \quad 001661001652 \quad 001703 \quad 001678 \quad 001151$ $001708 \quad 001702 \quad 001665 \quad 001619001514 \quad 001604 \quad 001648 \quad 001663001159$ $\begin{array}{llllllllll}0 & 01665 & 001787 & 001702 & 001724 & 001632 & 001718 & 001734 & 001835 & 001167\end{array}$ $001805 \quad 001849001678 \quad 001683 \quad 001649001650 \quad 001648 \quad 001623001175$ $001521001682001583 \quad 001691 \quad 001587 \quad 001599001593 \quad 001708001183$ $001680001603 \quad 001649001572001660 \quad 001602001636001608001191$ $001547001629001678 \quad 001708 \quad 001559001628001588001650 \quad 001199$ $001612001612 \quad 001664 \quad 001628 \quad 001658 \quad 001620001697 \quad 001676001207$ $001579001660 \quad 001546 \quad 001631 \quad 001506 \quad 0016 C 7 \quad 001618001709001215$ $001572001699001621001632001511001609001583 \quad 001670 \quad 001223$ $001605001709001756 \quad 001722 \quad 001631001772 \quad 001825001836001231$ $\begin{array}{llllllllll}001787 & 001677 & 001743 & 001563 & 001612 & 001584 & 001587 & 001619 & 001239\end{array}$ $\begin{array}{lllllllllll}0.01566 & 001626 & 001618 & 001519 & 001567 & 001538 & 001616 & 001633 & 001247\end{array}$ 001582001642001622001558001543001595001572001561001255 $001558 \quad 001511 \quad 001629001640 \quad 001526 \quad 001561001580 \quad 001589001263$ $001501001610 \quad 001625001513 \quad 001620 \quad 001605 \quad 001488001651001271$ $001483001558 \quad 001492 \quad 001560 \quad 001495 \quad 001588 \quad 001581001576 \quad 001279$

$40-0001$
$40-0002$
$40-0003$
$40-0004$
$40-0005$
$40-0006$
$40-0007$
$40-0008$
$40-0009$
$40-0010$
$40-0011$
$40-0012$
$40-0013$
$40-0014$
$40-0015$
$40-0016$
$40-0017$
$40-0018$
$40-0019$
$40-0020$
$40-0021$
$40-0022$
$40-0023$
$40-0024$
$40-0025$
$40-0026$
$40-0027$
$40-0028$
$40-0029$
$40-0030$
$40-0031$
$40-0032$ 
Appendix I

GAMANL OUTPUT DATA AND THEIR SPECTRAL FIGURES

OF RUNS NO. 99, 102, 103, 104,AND 105

PKAN Original data plots done by the CAL-COMP at the MIT Information Processing Center follow immediately after. the data.

Runs No. 99, 102, and 103 are decay gamma-ray spectra in Blanket No. 2. The identifying characteristics of these gamma-ray spectra is given in Table $I-I$.

TABLE I-I

DESCRIP'LIUNS OF RUNS NO. 99, 102, and 103

\begin{tabular}{cccc}
\hline Run Number & 99 & 102 & 103 \\
\hline $\begin{array}{c}\text { Irradiation Time } \\
\text { (min.) }\end{array}$ & 1424 & 1372 & 1372 \\
\hline $\begin{array}{c}\text { Cooling Time } \\
\text { (min.) }\end{array}$ & 106 & 288 & 1509 \\
\hline $\begin{array}{c}\text { Counting Time } \\
\text { (min.) }\end{array}$ & 949 & 1187 & 2589 \\
\hline $\begin{array}{c}\text { Diameter of Collimator } \\
\text { (inch) }\end{array}$ & None None & None \\
\hline
\end{tabular}

Runs No. 104 and 105 are prompt gamma-ray spectra in Blanket No. 2. The description of these runs is given in Table I-2. 
TABLE I-2

DESCRIPTIONS OF RUNS NO. 104 and 105

\begin{tabular}{ccc}
\hline Run Number & 104 & 105 \\
\hline $\begin{array}{c}\text { Irradiation Time } \\
\text { (min.) }\end{array}$ & 1380 & 1380 \\
\hline $\begin{array}{c}\text { Diameter of } \\
\text { Colimators } \\
\text { (inch) }\end{array}$ & $5 / 16$ & $3 / 16$ \\
\hline
\end{tabular}

Run numbers and line numbers are labeled at the end of printing lines. 


$\begin{array}{rrrrrr}\text { NO. } & \text { ENERGY(KEV) } & \text { INTIGAUS) } & \text { PC ERR } & \text { AREAG } & \text { AREAS } \\ 1 & 213.179 & 0.0108 & 10.215 & 2185.908 & 1803.234 \\ 2 & 230.928 & 0.053 & 2.082 & 12710.273 & 12485.074 \\ 4 & 251.546 & 0.005 & 16.975 & 1032.542 & 1297.477 \\ 5 & 264.872 & 0.003 & 29.531 & 548.363 & 411.492 \\ 9 & 296.527 & 0.004 & 18.232 & 764.832 & 618.344 \\ 9 & 304.299 & 0.005 & 17.073 & 796.529 & 565.570 \\ 10 & 317.174 & 0.014 & 6.321 & 2311.401 & 3017.425 \\ 11 & 335.219 & 0.018 & 5.610 & 2581.519 & 2478.785 \\ 14 & 378.109 & 3.005 & 20.575 & 534.859 & 367.550 \\ 15 & 385.227 & 0.003 & 36.900 & 287.285 & 178.970 \\ 3 & 240.598 & 0.007 & 10.953 & 1708.567 & 1399.023 \\ 6 & 279.294 & 0.094 & 1.469 & 18275.953 & 18789.246 \\ 7 & 287.872 & 0.009 & 8.936 & 1722.252 & 1244.609 \\ 12 & 350.438 & 0.003 & 33.040 & 351.183 & 205.263 \\ 13 & 371.009 & 0.003 & 26.784 & 410.167 & 234.385 \\ 17 & 511.000 & 0.023 & 6.743 & 1622.857 & 1756.907 \\ 16 & 435.124 & 0.003 & 31.287 & 317.083 & 276.097 \\ 18 & 530.557 & 0.015 & 9.930 & 1002.059 & 1112.492 \\ 19 & 541.595 & 0.004 & 35.640 & 249.631 & 158.561 \\ 20 & 556.301 & 0.011 & 14.156 & 641.306 & 671.860 \\ 21 & 585.002 & 0.010 & 15.488 & 552.237 & 489.808 \\ 22 & 592.583 & 0.007 & 21.206 & 384.741 & 374.947 \\ 23 & 658.195 & 0.033 & 6.343 & 1534.855 & 1861.594 \\ 24 & 667.134 & 0.014 & 12.589 & 655.797 & 797.844 \\ 25 & 725.275 & 0.006 & 31.917 & 222.122 & 261.589 \\ 26 & 743.282 & 0.024 & 8.956 & 948.259 & 1014.743 \\ 27 & 761.174 & 0.007 & 26.548 & 258.653 & 224.519 \\ 28 & 772.207 & 0.019 & 11.094 & 703.500 & 607.910 \\ 29 & 811.786 & 0.009 & 22.388 & 300.782 & 265.597 \\ 30 & 816.542 & 0.008 & 24.367 & 273.771 & 232.267 \\ 31 & 824.820 & 0.010 & 19.604 & 343.249 & 425.135 \\ 32 & 831.531 & 0.012 & 17.416 & 392.570 & 497.056 \\ 33 & 846.776 & 0.065 & 4.816 & 2071.415 & 2552.007 \\ 34 & 912.462 & 0.007 & 31.253 & 208.065 & 134.707 \\ 35 & 946.443 & 0.007 & 34.137 & 187.467 & 112.623\end{array}$

$99-0001$
$99-0002$
$99-0003$
$99-0004$
$99-0005$
$99-0006$
$99-0006$
$99-0007$
$99-0008$
$99-0009$
$99-0010$
$99-0011$
$99-0012$
$99-0013$
$99-0014$
$99-0015$
$99-0016$
$99-0017$
$99-0018$
$99-0019$
$99-0020$
$99-0021$
$99-0022$
$99-0023$
$99-0024$
$99-0025$
$99-0026$
$99-0027$
$99-0028$
$99-0029$
$99-0030$
$99-0031$
$99-0032$
$99-0033$
$99-0034$
$99-0035$
$99-0036$




$\begin{array}{rrrrrr}36 & 961.593 & 0.008 & 29.805 & 217.064 & 193.209 \\ 37 & 1024.770 & 0.015 & 19.289 & 354.856 & 328.490 \\ 38 & 1039.246 & 0.011 & 25.730 & 254.732 & 157.101 \\ 39 & 1076.386 & 0.012 & 23.993 & 270.727 & 197.927 \\ 40 & 1147.574 & 0.010 & 28.750 & 192.113 & 144.234 \\ 41 & 1173.098 & 0.066 & 6.328 & 1245.071 & 1352.769 \\ 42 & 1261.327 & 0.016 & 17.082 & 279.039 & 207.467 \\ 43 & 1282.542 & 0.009 & 27.577 & 148.538 & 155.771 \\ 44 & 1293.600 & 0.221 & 3.205 & 3641.353 & 3719.141 \\ 45 & 1332.499 & 0.063 & 6.735 & 987.227 & 1029.501 \\ 46 & 1368.656 & 0.166 & 3.924 & 2508.201 & 2613.879 \\ 47 & 1384.916 & 0.016 & 17.662 & 232.396 & 208.447 \\ 48 & 1408.287 & 0.008 & 32.237 & 107.772 & 68.135 \\ 49 & 1451.560 & 0.011 & 24.171 & 144.621 & 155.647 \\ 50 & 1460.339 & 0.070 & 6.748 & 935.603 & 993.641 \\ 51 & 1509.873 & 0.007 & 34.670 & 93.760 & 89.726 \\ 52 & 1522.522 & 0.010 & 26.256 & 127.285 & 80.339 \\ 53 & 1587.584 & 0.010 & 29.591 & 113.215 & 106.813 \\ 54 & 1595.705 & 0.021 & 16.584 & 238.908 & 243.432 \\ 55 & 1677.864 & 0.010 & 30.932 & 104.157 & 55.147 \\ 56 & 1731.830 & 0.177 & 4.693 & 1815.599 & 2004.511 \\ 57 & 1758.910 & 0.011 & 28.007 & 114.223 & 61.037 \\ 58 & 1780.660 & 0.013 & 25.569 & 124.087 & 140.891 \\ 59 & 1794.482 & 0.013 & 24.632 & 130.241 & 149.747 \\ 60 & 1810.563 & 0.033 & 13.104 & 315.250 & 361.954 \\ 61 & 1875.021 & 0.016 & 23.338 & 140.300 & 100.923 \\ 62 & 1938.818 & 0.014 & 25.580 & 121.382 & 111.408 \\ 63 & 2208.377 & 0.019 & 24.802 & 122.236 & 114.710 \\ 64 & 2243.532 & 0.041 & 14.818 & 266.767 & 405.016 \\ 65 & 2320.183 & 0.019 & 25.773 & 123.6 .28 & 110.379 \\ 66 & 2614.956 & 0.021 & 21.751 & 121.774 & 95.720 \\ 67 & 2754.157 & 0.235 & 5.844 & 1320.215 & 1404.004\end{array}$

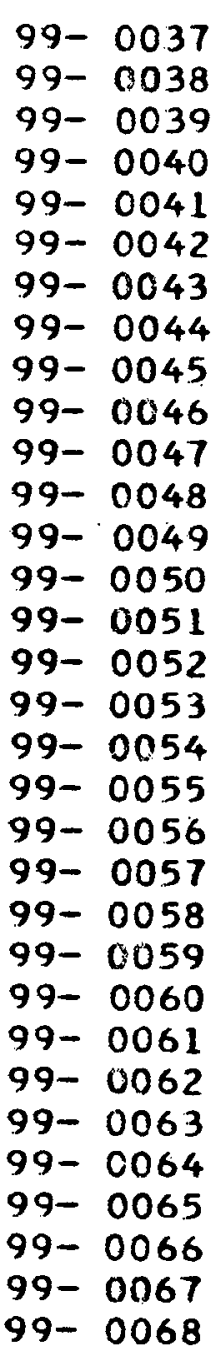

$\frac{4}{4}$ 


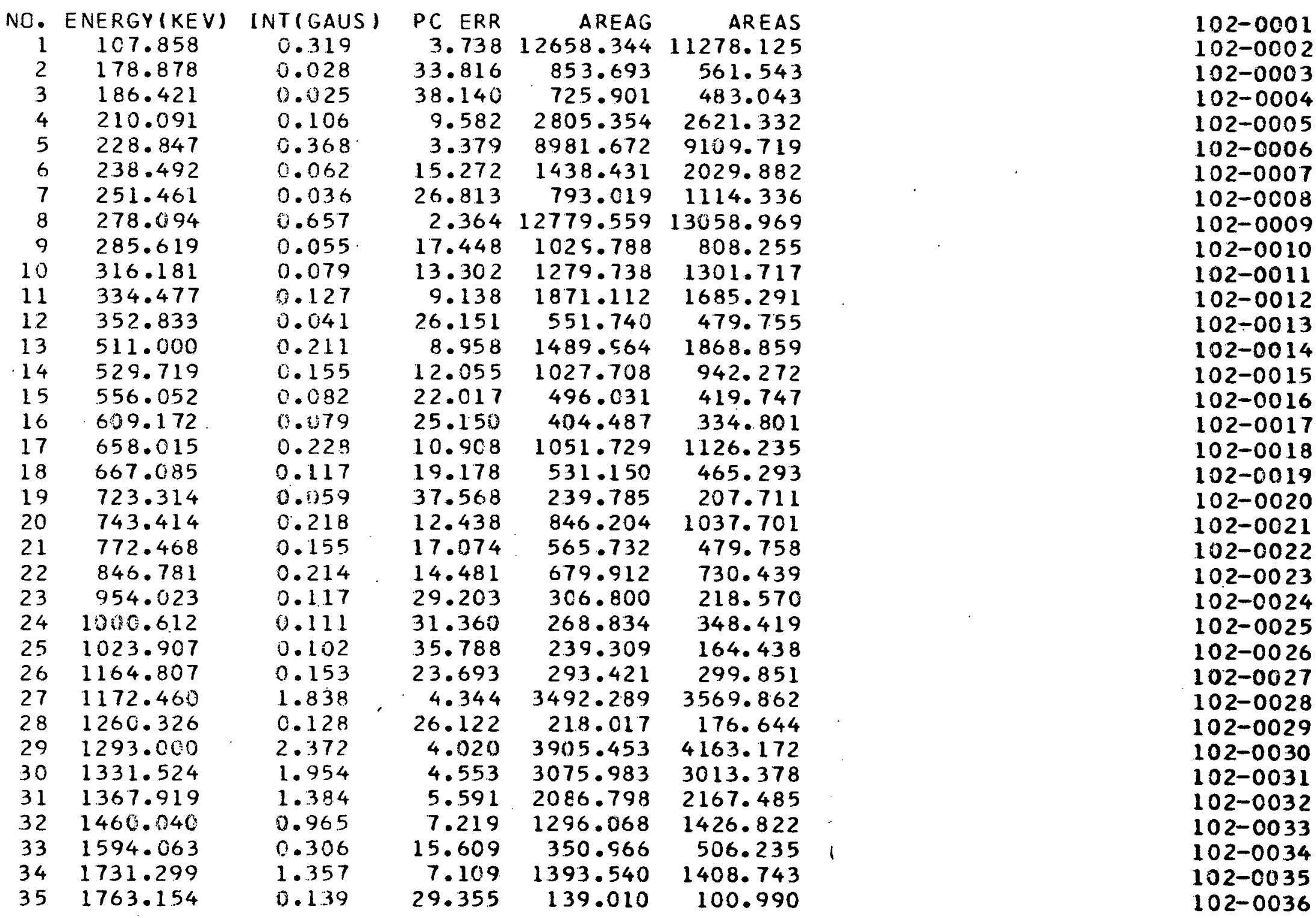




$\begin{array}{rrrrrr}36 & 1810.703 & 0.185 & 24.946 & 177.481 & 146.414 \\ 37 & 2240.785 & 0.314 & 23.599 & 204.104 & 196.744 \\ 38 & 2613.130 & 0.220 & 28.986 & 128.471 & 107.409 \\ 39 & 2751.601 & 2.113 & 8.313 & 1185.930 & 1262.056\end{array}$




$\begin{array}{rrrrrr}\text { NO. } & \text { ENERGY(KEV) } & \text { INI(GAUS } & \text { PC ERR } & \text { AREAG } & \text { AREAS } \\ 1 & 108.594 & 0.483 & 3.219 & 19112.602 & 16959.477 \\ 2 & 117.238 & 0.069 & 19.644 & 2628.319 & 1732.488 \\ 3 & 210.687 & 0.170 & 7.787 & 4488.375 & 4189.754 \\ 4 & 229.332 & 0.577 & 2.792 & 14042.230 & 14680.539 \\ 5 & 239.324 & 0.165 & 7.940 & 3836.449 & 4358.730 \\ 6 & 270.759 & 0.047 & 26.216 & 947.367 & 694.797 \\ 7 & 278.464 & 0.965 & 2.032 & 18747.805 & 19007.934 \\ 8 & 286.107 & 0.078 & 15.947 & 1467.226 & 936.248 \\ 9 & 296.432 & 0.052 & 23.593 & 933.570 & 1402.842 \\ 10 & 317.845 & 0.135 & 10.237 & 2157.691 & 3232.302 \\ 11 & 334.925 & 0.163 & 9.034 & 2384.022 & 2393.234 \\ 12 & 353.092 & 0.075 & 18.836 & 994.345 & 837.214 \\ 13 & 377.540 & 0.038 & 37.780 & 444.695 & 287.482 \\ 14 & 486.014 & 0.071 & 26.116 & 546.845 & 472.229 \\ 15 & 511.000 & 0.232 & 9.616 & 1636.404 & 2288.453 \\ 16 & 529.241 & 0.122 & 17.401 & 808.996 & 701.763 \\ 17 & 558.240 & 0.058 & 36.552 & 347.389 & 217.407 \\ 18 & 582.955 & 0.119 & 19.981 & 651.587 & 548.469 \\ 19 & 608.826 & 0.163 & 15.700 & 828.796 & 824.666 \\ 20 & 657.686 & 0.204 & 13.570 & 942.732 & 1096.937 \\ 21 & 666.913 & 0.236 & 12.201 & 1069.776 & 1261.304 \\ 22 & 743.211 & 0.173 & 17.722 & 669.804 & 551.592 \\ 23 & 771.864 & 0.291 & 11.872 & 1064.555 & 871.063 \\ 24 & 909.753 & 0.271 & 15.138 & 766.142 & 1806.896 \\ 25 & 947.162 & 0.223 & 18.876 & 592.073 & 1398.600 \\ 26 & 953.553 & 0.206 & 20.482 & 539.235 & 1273.746 \\ 27 & 1112.481 & 0.276 & 17.667 & 569.922 & 898.044 \\ 28 & 1170.832 & 4.510 & 2.709 & 8589.191 & 8939.211 \\ 29 & 1286.564 & 0.112 & 30.599 & 186.500 & 151.564 \\ 30 & 1329.505 & 5.076 & 2.761 & 8007.551 & 8325.996 \\ 31 & 1337.234 & 0.123 & 26.711 & 191.632 & 85.425 \\ 32 & 1365.532 & 0.935 & 7.006 & 1413.162 & 1466.850 \\ 33 & 1457.761 & 2.169 & 4.655 & 2922.282 & 3189.874 \\ 34 & 1591.575 & 0.618 & 10.011 & 711.620 & 1181.397 \\ 35 & 1727.941 & 0.974 & 8.275 & 1002.937 & 1205.884\end{array}$

103-0001

103-0002

103-0003

103-0004

103-0005

103-0006

103-0007

103-0008

103-0009

103-0010

103-0011

103-0012

103-0013

$103-0014$

103-0015

$103-0016$

$103-0017$

103-0018

103-0019

103-0020

103-0021

103-0022

103-0023

103-0024

103-0025

103-0026

103-0027

103-0028

103-0029

$103-0030$

103-0031

103-0032

103-0033

103-0034

103-0035

103-0036 


$\begin{array}{llllll}36 & 2237.318 & 0.227 & 27.567 & 147.612 & 150.204 \\ 37 & 2607.338 & 0.503 & 16.779 & 294.811 & 301.718 \\ 38 & 2746.359 & 1.258 & 11.630 & 707.311 & 778.368\end{array}$

103-0037 $103-0038$

103-0039 


$\begin{array}{rcc}\text { NO. ENERGY(KEV) } & \text { INT(GAUS) } \\ 1 & 459.106 & 0.014 \\ 2 & 477.202 & 0.049 \\ 3 & 493.330 & 0.089 \\ 4 & 511.000 & 0.939 \\ 5 & 529.796 & 0.146 \\ 6 & 546.501 & 0.096 \\ 7 & 596.450 & 0.275 \\ 8 & 627.373 & 0.022 \\ 9 & 644.570 & 0.022 \\ 10 & 662.763 & 0.022 \\ 11 & 695.240 & 0.275 \\ 12 & 812.257 & 0.025 \\ 13 & 832.721 & 0.094 \\ 14 & 846.946 & 0.102 \\ 15 & 867.458 & 0.111 \\ 16 & 886.066 & 0.038 \\ 17 & 902.678 & 0.036 \\ 18 & 959.601 & 0.043 \\ 19 & 996.144 & 0.047 \\ 20 & 1016.647 & 0.102 \\ 21 & 1038.990 & 0.176 \\ 22 & 1066.548 & 0.278 \\ 23 & 1173.157 & 0.156 \\ 24 & 1200.894 & 0.118 \\ 25 & 1221.134 & 0.033 \\ 26 & 1259.855 & 0.058 \\ 27 & 1275.950 & 0.244 \\ 28 & 1293.600 & 2.935 \\ 29 & 1311.706 & 0.342 \\ 30 & 1331.080 & 0.375 \\ 31 & 1346.816 & 0.200 \\ 32 & 1588.797 & 0.040 \\ 33 & 1728.436 & 0.070 \\ 34 & 1780.983 & 0.061 \\ 35 & 2225.275 & 0.363 \\ & & \end{array}$

$\begin{array}{rrr}P C \text { ERR } & \text { AREAG } & \text { AREAS } \\ 33.872 & 1145.985 & 804.066 \\ 9.989 & 3938.426 & 3234.523 \\ 5.961 & 6677.461 & 4220.941 \\ 0.996 & 66222.750 & 56928.500 \\ 3.958 & 9628.043 & 15835.121 \\ 6.002 & 5985.398 & 9879.695 \\ 2.8319 & 14432.184 & 23677.652 \\ 29.980 & 1083.390 & 757.431 \\ 30.526 & 1044.133 & 776.910 \\ 30.577 & 1025.351 & 993.633 \\ 3.206 & 11735.730 & 20149.182 \\ 33.600 & 851.739 & 738.270 \\ 9.780 & 3049.276 & 3385.102 \\ 5.285 & 3225.923 & 3611.931 \\ 8.792 & 3386.718 & 3226.375 \\ 25.029 & 1119.021 & 1099.067 \\ 26.932 & 1037.552 & 1034.893 \\ 24.896 & 1111.435 & 991.355 \\ 24.098 & 1142.966 & 957.023 \\ 11.126 & 2418.334 & 5632.043 \\ 6.991 & 4 C 32.968 & 9390.566 \\ 4.876 & 6114.258 & 14234.102 \\ 8.043 & 2569.536 & 2951.829 \\ 10.372 & 2155.152 & 2419.078 \\ 35.105 & 582.835 & 446.753 \\ 20.424 & 993.602 & 917.462 \\ 5.492 & 4097.801 & 2759.040 \\ 1.038 & 48282.281 & 48718.508 \\ 4.043 & 5513.652 & 18504.961 \\ 3.840 & 5907.156 & 20438.359 \\ 6.383 & 3087.626 & 10674.215 \\ 35.208 & 465.593 & 494.744 \\ 22.042 & 722.646 & 633.059 \\ 25.746 & 601.157 & 537.754 \\ 6.589 & 2366.207 & 2509.977\end{array}$

104-0001 $104-0012$ $124-10003$ 104-0004 $104-0005$ $104-0006$ $104-000 ?$ $104-0003$ $104-0009$ 104-0010 $104-0011$ 104-0012 $104-0913$ 104-0014 $104-0015$ 104-0016 104-0017 104-0018 104-0019 104-0020 $104-0021$ 104-002.2 104-0023 $104-0024$ 104-0025 104-0026 104-0027 104-0028 104-0029 104-0030 104-0031 104-0032 104-0033 104-0034 104-0035 104-0036 


$\begin{array}{rllllr}36 & 2391.301 & 0.073 & 25.784 & 455.274 & 512.573 \\ 37 & 2828.953 & 0.057 & 32.534 & 314.547 & 433.933 \\ 38 & 3201.317 & 0.123 & 17.184 & 592.713 & 553.019 \\ 39 & 3388.760 & 0.077 & 26.340 & 347.753 & 304.936 \\ 40 & 3709.047 & 0.069 & 31.087 & 270.374 & 392.802 \\ 41 & 3794.903 & 0.067 & 33.061 & 254.578 & 204.502 \\ 42 & 3929.173 & 0.092 & 25.382 & 339.277 & 321.601 \\ 43 & 4905.406 & 0.519 & 7.641 & 1794.648 & 2203.894 \\ 44 & 5003.273 & 0.458 & 8.305 & 1583.407 & 1598.050 \\ 45 & 5181.020 & 0.058 & 35.610 & 199.871 & 139.861 \\ 46 & 5365.992 & 0.121 & 19.483 & 417.134 & 330.225 \\ 47 & 5419.438 & 0.067 & 31.111 & 232.200 & 194.890 \\ 48 & 5513.121 & 0.085 & 25.346 & 293.007 & 328.364 \\ 49 & 6266.563 & 0.172 & 17.244 & 595.818 & 716.847 \\ 50 & 6619.016 & 1.004 & 14.283 & 3469.503 & 4073.336 \\ 51 & 6631.465 & 0.765 & 14.528 & 2644.042 & 3100.258 \\ 52 & 6710.379 & 0.208 & 18.665 & 717.824 & 764.592 \\ 53 & 7130.902 & 0.155 & 22.997 & 536.525 & 1005.655 \\ 54 & 7645.598 & 0.061 & 32.750 & 210.083 & 330.517 \\ 55 & 7874.633 & 0.040 & 38.944 & 136.763 & 202.895 \\ 56 & 8286.094 & 0.088 & 40.916 & 304.016 & 288.207 \\ 57 & 9410.453 & 0.031 & 71.868 & 106.301 & 35.201 \\ 58 & 9419.777 & 0.038 & 71.184 & 130.607 & 41.234 \\ 59 & 9428.664 & 0.059 & 69.929 & 202.943 & 67.456 \\ 60 & 9437.887 & 0.0381 & 69.462 & 279.650 & 91.339\end{array}$

$104-9037$

104-0r33

$104-0039$

$104-0040$

104-0041

104-0042

$104-0043$

104-0044

104-0045

10.4-0046

$104-0047$

104-0048

104-0049

$104-0050$

104-0051

104-0052

104-0053

104-0054

104-0055

$104-0056$

104-0057

104-0058

104-0059

104-0060

104-00B1 


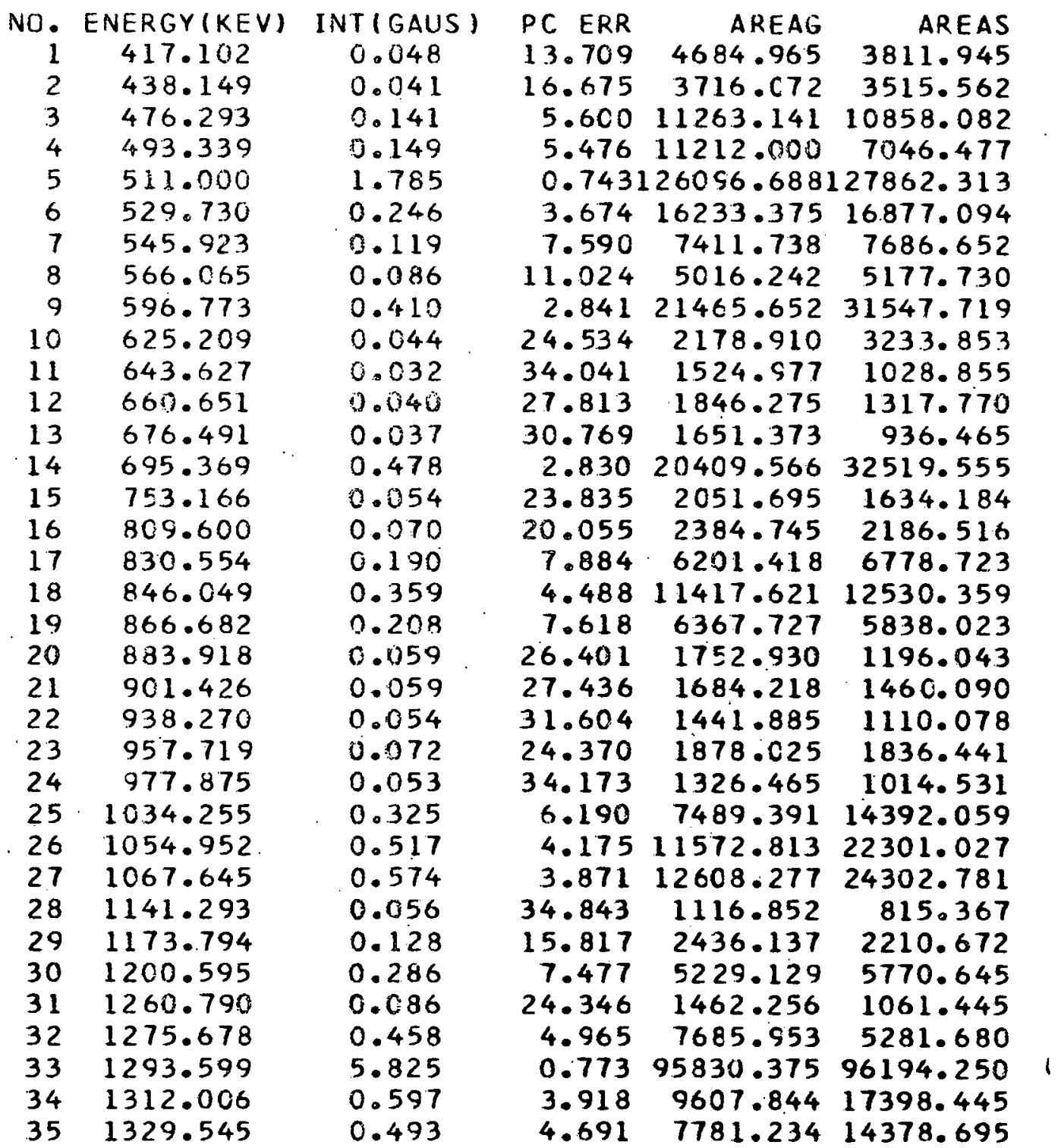

$105-0001$
$105-0002$
$105-0003$
$105-0004$
$105-0005$
$105-0006$
$105-0007$
$105-0008$
$105-0009$
$105-0010$
$105-0011$
$105-0012$
$105-0013$
$105-0014$
$105-0015$
$105-0016$
$105-0017$
$105-0018$
$105-0019$
$105-0020$
$105-0021$
$105-0022$
$105-0023$
$105-0024$
$105-0025$
$105-0026$
$105-0027$
$105-0028$
$105-0029$
$105-0030$
$105-0031$
$105-0032$
$105-0033$
$105-0034$
$105-0035$
$105-0036$




$\begin{array}{lll}36 & 1362.635 & 0.280 \\ 37 & 1433.933 & 0.169 \\ 38 & 1459.761 & 0.148 \\ 39 & 1546.060 & 0.070 \\ 40 & 1565.016 & 0.083 \\ 41 & 1615.142 & 0.114 \\ 42 & 1658.895 & 0.085 \\ 43 & 1676.159 & 0.085 \\ 44 & 1694.106 & 0.084 \\ 45 & 1712.429 & 0.139 \\ 46 & 1728.494 & 0.285 \\ 47 & 1749.065 & 0.093 \\ 48 & 1780.853 & 0.262 \\ 49 & 2206.265 & 0.118 \\ 50 & 2224.811 & 0.894 \\ 51 & 2242.717 & 0.143 \\ 52 & 2334.394 & 0.103 \\ 53 & 2352.069 & 0.107 \\ 54 & 2390.025 & 0.150 \\ 55 & 2418.660 & 0.107 \\ 56 & 2572.258 & 0.127 \\ 57 & 2756.356 & 0.198 \\ 58 & 3115.814 & 0.116 \\ 59 & 3138.850 & 0.137 \\ 60 & 3173.018 & 0.105 \\ 61 & 3198.591 & 0.419 \\ 62 & 3388.608 & 0.198 \\ 63 & 3444.674 & 0.187 \\ 64 & 3793.810 & 0.257 \\ 65 & 3863.431 & 0.119 \\ 66 & 3884.296 & 0.110 \\ 67 & 3929.704 & 0.403 \\ 68 & 4227.730 & 0.144 \\ 69 & 4510.117 & 0.177 \\ 70 & 4904.563 & 1.359 \\ 71 & 4949.297 & 0.127\end{array}$

8.055
14.042
16.431
35.983
30.895
23.387
31.254
31.585
32.253
19.918
10.210
29.914
11.325
29.790
4.768
24.703
33.640
32.253
23.474
32.491
27.395
17.950
30.831
26.239
33.818
9.592
19.533
20.692
16.748
34.403
37.081
11.396
28.981
23.014
4.443
29.659

4244.570

2344.647

1983.029

850.533

979.491

1285.466

926.239

910.679

887.369

1445.831

2937.366

940.388

2580.231

775.973

5830.555

929.709

650.070

675.756

936.441

661.987

753.227

1107.347

578.524

676.434

515.365

2027.268

892.370

823.701

978.871

444.311

410.288

1485.245

498.411

611.121

4696.270

438.659
7786.570

2114.301

2066.344

622.398

792.594

1041.934

692.707

673.961

670.344

14.96 .601

3043.073

720.344

2438.645

545.992

5468.355

617.422

477.921

457.131

892.917

757.961

656.872

905.167

509.044

529.914

377.698

2446.778

870.829

864.017

1008.996

609.046

385.396

1706.624

908.489

741.236

5694.051

727.118
105-0037

105-0038

105-0039

105-0040

105-0041

105-0042

105-0043

105-0044

105-0045

105-0046

105-0047

105-0048

105-0049

105-0050

105-0051

105-0052

105-0053

105-0054

105-0055

105-0056

105-0057

105-0058

105-0059

105-0060

105-0061

105-0062

105-0063

105-0064

105-0065

105-0066

105-0067

105-0068

105-0069

105-0070

105-0071

105-0072 


$\begin{array}{rrrrrr}72 & 5002.707 & 1.316 & 4.555 & 4548.410 & 5443.262 \\ 73 & 5053.020 & 0.115 & 32.129 & 397.463 & 378.422 \\ 74 & 5076.387 & 0.127 & 29.253 & 438.831 & 574.279 \\ 75 & 5091.199 & 0.236 & 16.718 & 814.146 & 1072.372 \\ 76 & 5236.230 & 0.178 & 21.439 & 614.725 & 508.369 \\ 77 & 5367.492 & 0.350 & 11.623 & 1209.005 & 1533.405 \\ 78 & 5381.563 & 0.162 & 22.595 & 559.650 & 711.659 \\ 79 & 5417.109 & 0.213 & 17.718 & 737.851 & 979.849 \\ 80 & 5514.445 & 0.176 & 20.783 & 609.300 & 564.425 \\ 81 & 5693.570 & 0.139 & 24.727 & 480.055 & 378.155 \\ 82 & 5767.234 & 0.124 & 27.230 & 427.656 & 501.934 \\ 83 & 6145.758 & 0.118 & 27.224 & 468.997 & 346.203 \\ 84 & 6228.984 & 0.184 & 18.415 & 634.417 & 1019.083 \\ 85 & 6264.684 & 0.599 & 7.725 & 2070.156 & 3383.674 \\ 86 & 6299.227 & 0.100 & 31.647 & 344.702 & 240.179 \\ 87 & 6350.875 & 0.123 & 26.435 & 424.899 & 536.964 \\ 88 & 6618.664 & 3.193 & 5.080 & 11035.141 & 15793.465 \\ 89 & 6630.148 & 2.469 & 5.267 & 8533.848 & 12244.852 \\ 90 & 6711.113 & 1.203 & 6.209 & 4156.922 & 5936.016 \\ 91 & 6772.184 & 0.132 & 24.383 & 457.168 & 578.554 \\ 92 & 6928.914 & 0.108 & 27.246 & 372.375 & 446.167 \\ 93 & 7131.711 & 0.512 & 9.211 & 1769.952 & 2405.972 \\ 94 & 7142.641 & 0.375 & 10.429 & 1257.652 & 1765.784 \\ 95 & 7221.762 & 0.193 & 14.704 & 667.512 & 842.306 \\ 96 & 7471.832 & 0.048 & 35.556 & 165.629 & 289.243 \\ 97 & 7643.750 & 0.155 & 14.885 & 535.448 & 1257.325 \\ 98 & 7730.398 & 0.058 & 25.399 & 201.787 & 195.259 \\ 99 & 7874.367 & 0.222 & 13.888 & 766.558 & 1237.986 \\ 100 & 8285.500 & 0.271 & 15.699 & 937.831 & 1499.682 \\ 101 & 8384.594 & 0.037 & 32.251 & 126.778 & 195.058 \\ 102 & 8523.141 & 0.026 & 36.535 & 91.565 & 66.424 \\ 103 & 8706.859 & 0.039 & 29.878 & 134.729 & 218.583 \\ 104 & 8794.793 & 0.046 & 27.809 & 159.517 & 193.156 \\ 105 & 9304.563 & 0.021 & 37.610 & 72.526 & 54.094 \\ 106 & 9358.992 & 0.020 & 38.345 & 70.260 & 32.448 \\ 107 & 9377.188 & 0.028 & 35.418 & 95.470 & 44.085 \\ & & & & & \end{array}$

$105-0073$
$105-0074$
$105-0075$
$105-0076$
$105-0077$
$105-0078$
$105-0079$
$105-0080$
$105-0081$
$105-0082$
$105-0083$
$105-0084$
$105-0085$
$105-0086$
$105-0087$
$105-0088$
$105-0089$
$105-0090$
$105-0091$
$105-0092$
$105-0093$
$105-0094$
$105-0095$
$105-0096$
$105-0097$
$105-0098$
$105-0099$
$105-0100$
$105-0101$
$105-0102$
$105-0103$
$105-0104$
$105-0105$
$105-0106$
$105-0107$
$105-0108$ 
1089386.293

1099395.434

1109404.265

1119413.488

1129422.270

1139431.379
0.0 .25

i). 040

0.042

0.059

0.063

0.086

36.470

32.677

32.587

31.086

31.058

30.163
85.940

139.382

146.139

202.861

219.084

296.619
36.755

64.550

64.376

91.715

91.949

124.337
105-0109

105-0110

105-0111

105-0112

105-0113

105-0.114 
GAMA-RAY ENERGY (WeV)

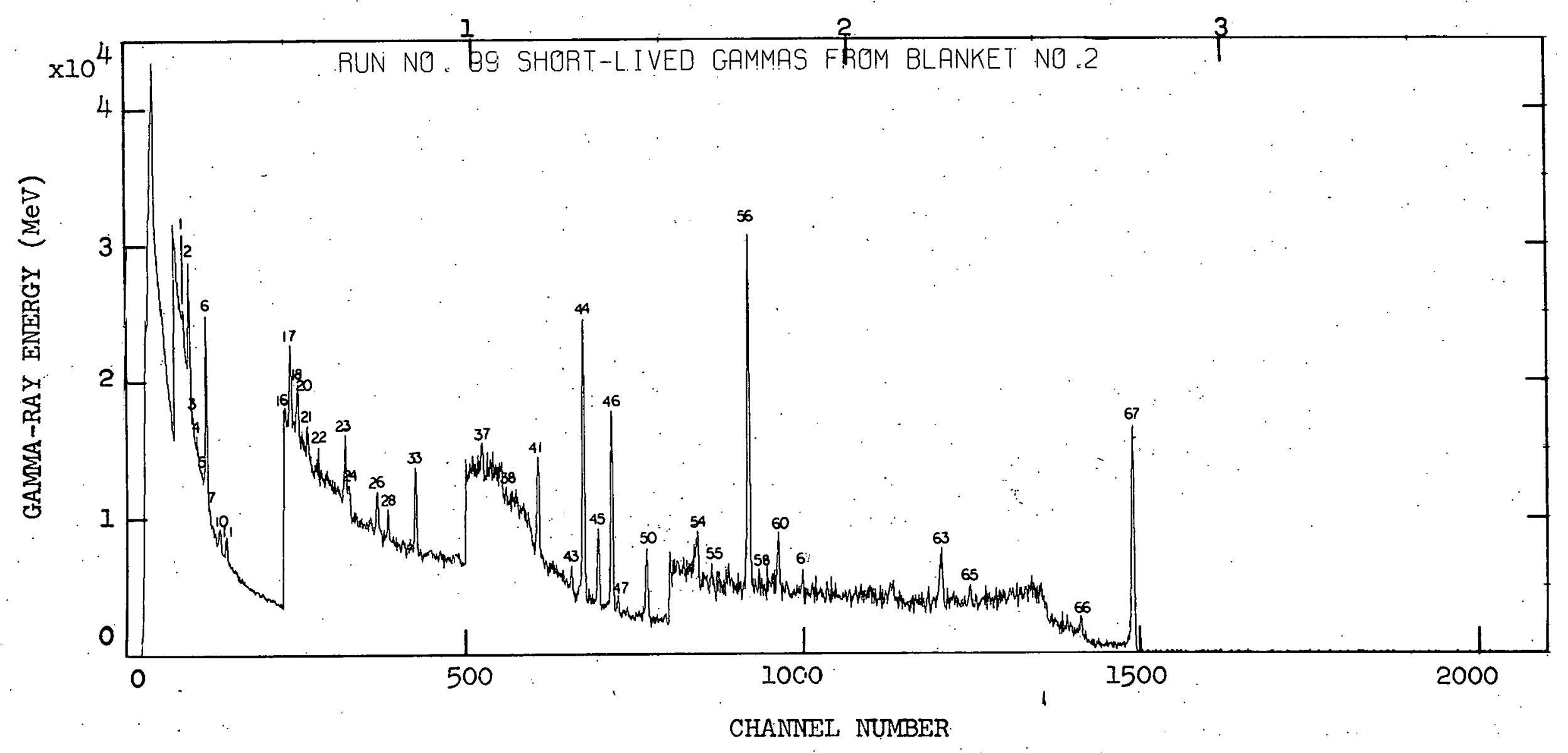

Fig. I-1 Run No. 99, Short-Lived Gamma-Rays from Elanke- No. 2 
엉

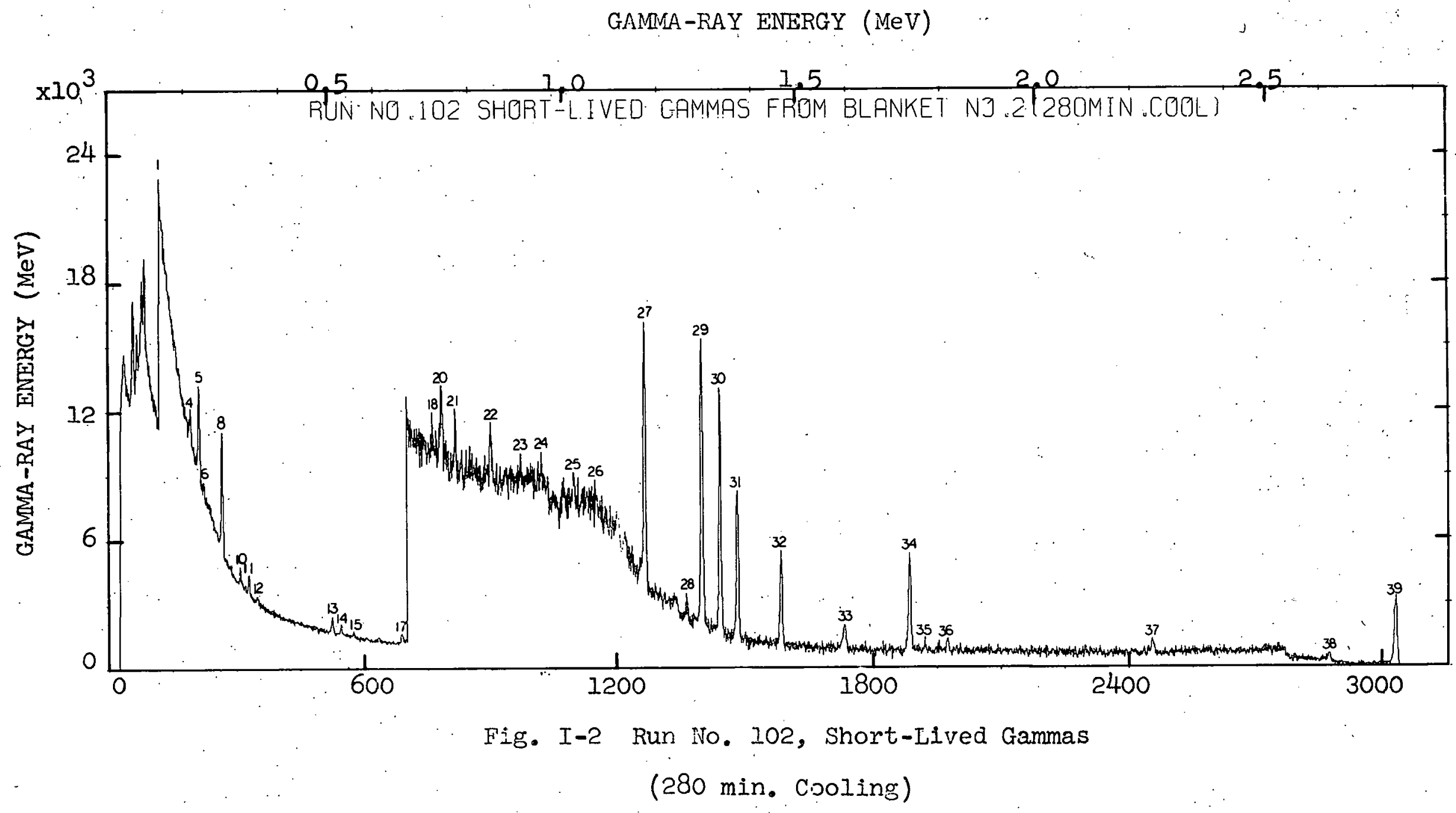


GAMPA-RAY ENERGY ( $(\mathrm{MeV})$

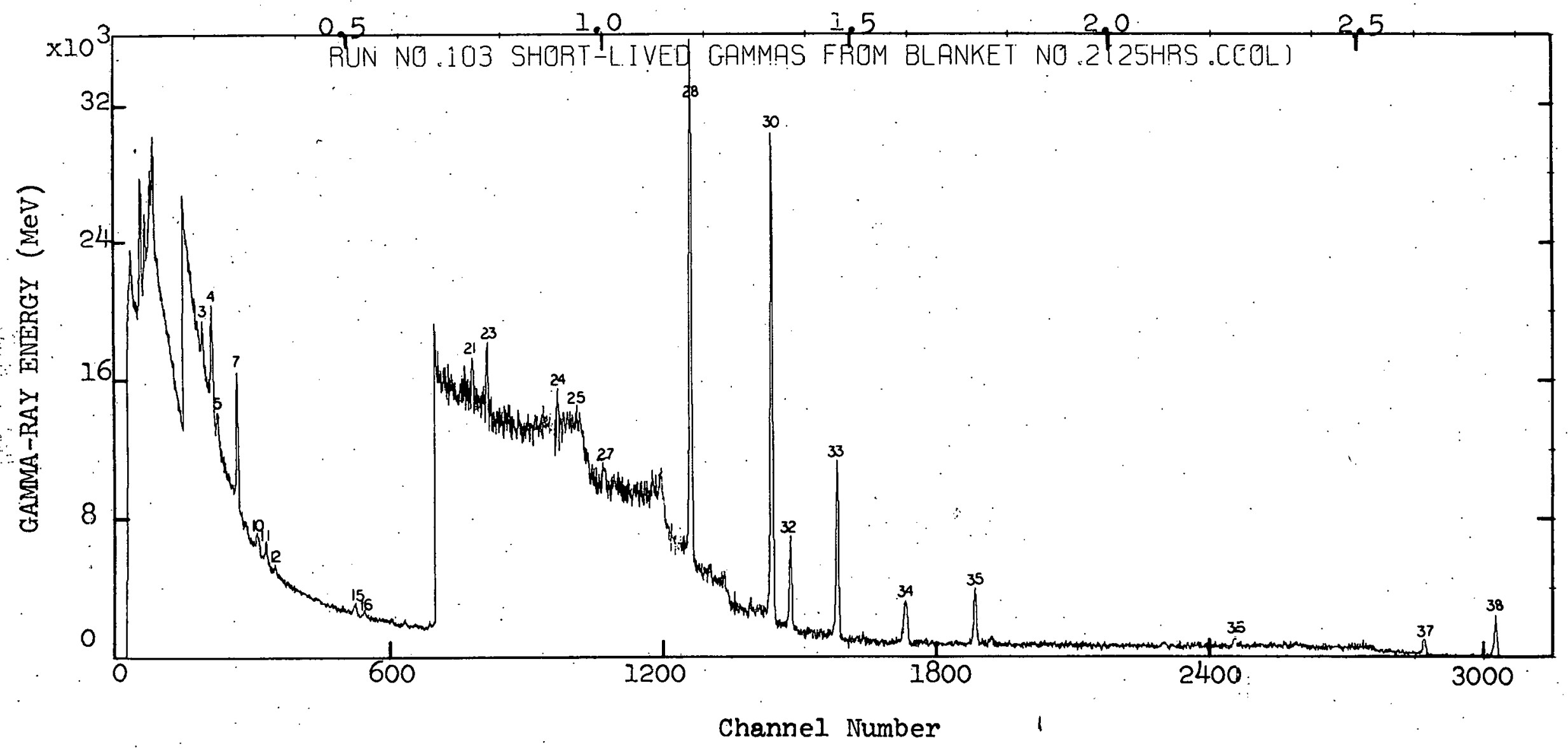

Fig. I-3 Run No. 103, Short-Lived Gammas

(25 Hrs. Cooling) 


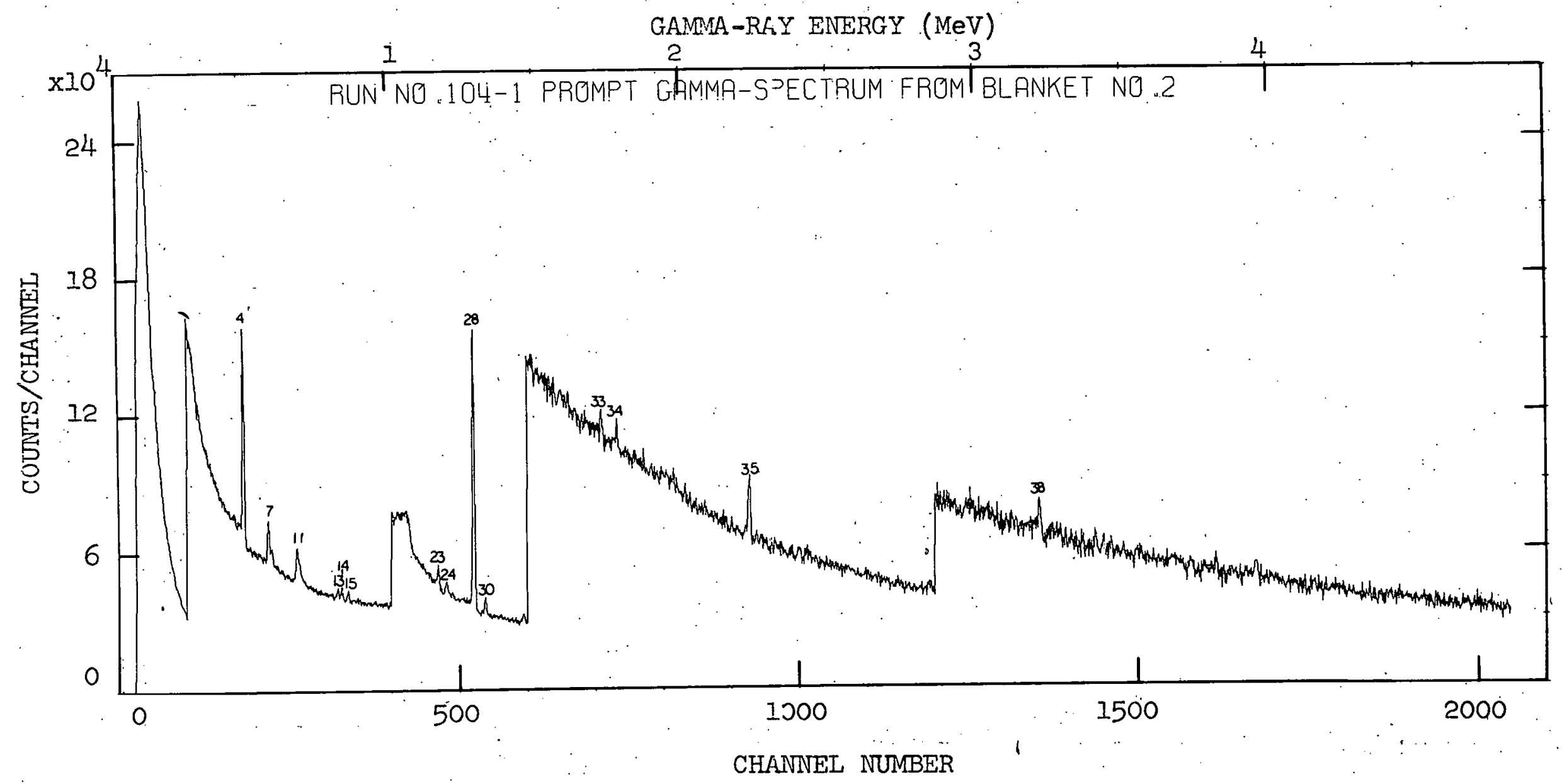

Fig. I-4' Run No. 104-1, Pro:mp̣t Gamma-Spectrum from Blanket No. 2 


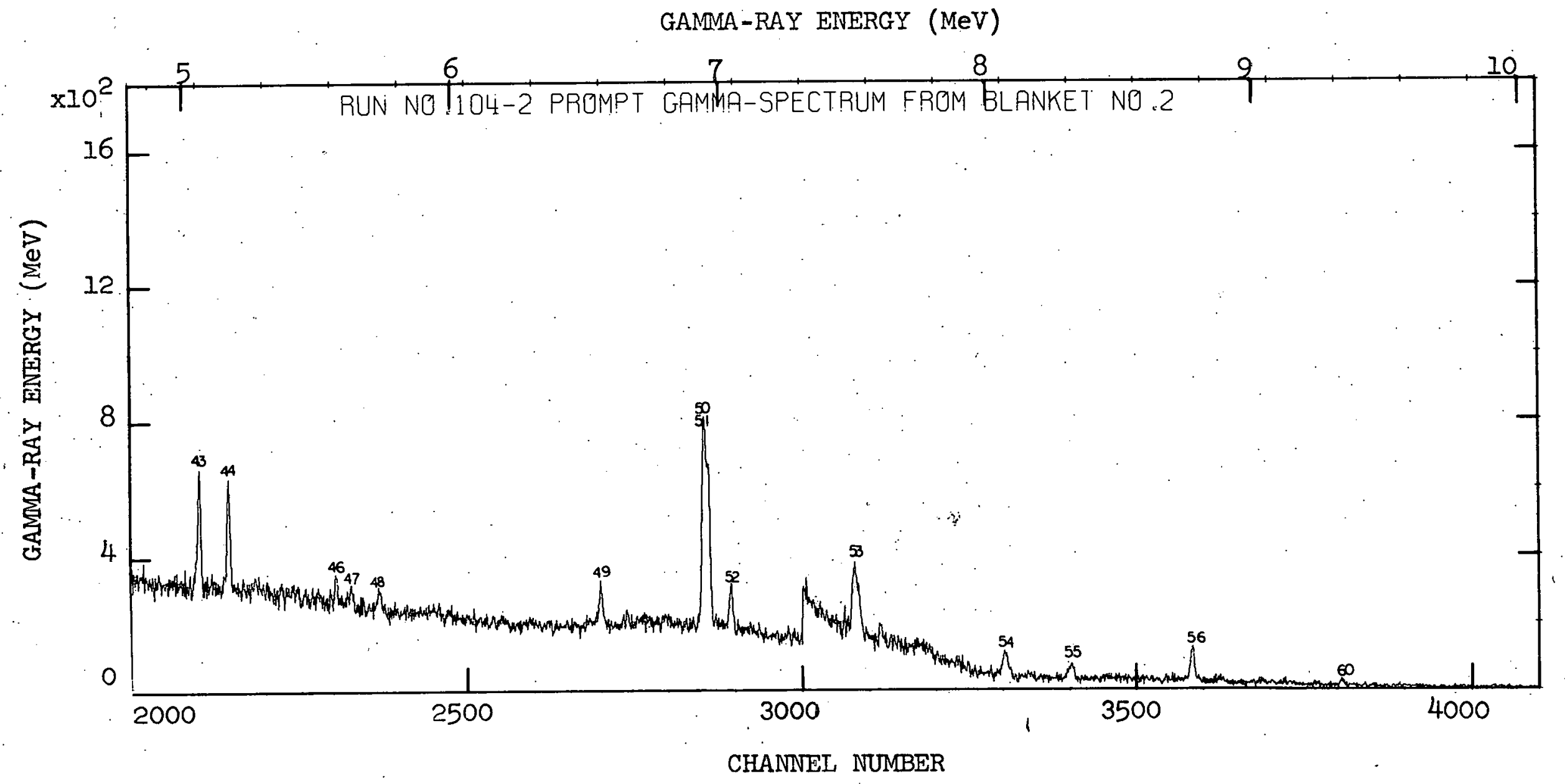

Fig. I-5 Run No. 104-2, Prompt Gamma-Spectrum from Blanket No. 2 
GAMA-RAY BNERGY (MeV)

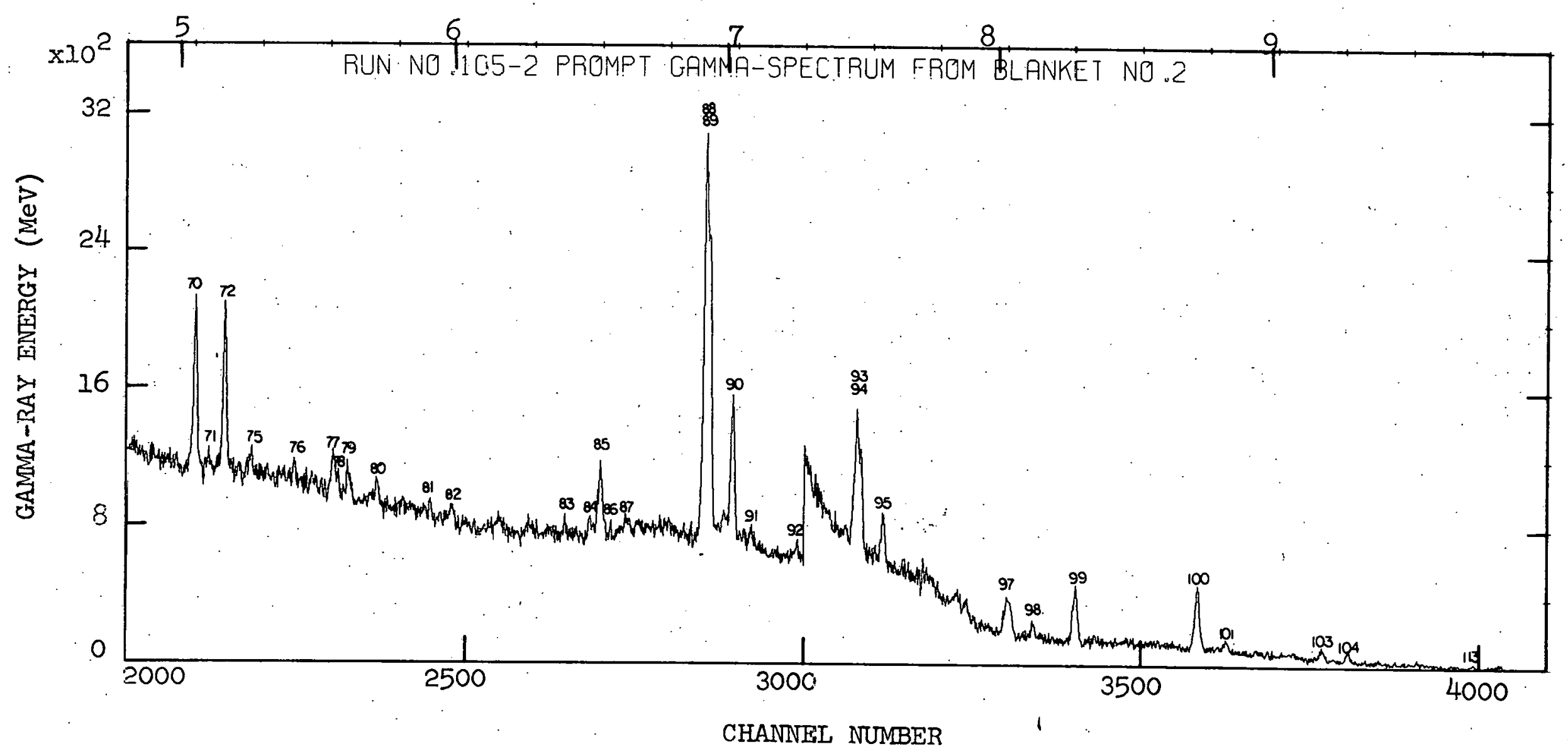

Fig. I-7 Run No. 105-2, Prompt Gamma-Spectrum from Blanket No. 2 